\title{
An Object Oriented, Finite Element Framework For Linear Wave Equations
}

\author{
Joseph M. Koning
}

Ph.D. Thesis 


\section{DISCLAIMER}

This document was prepared as an account of work sponsored by an agency of the United States Government. Neither the United States Government nor the University of California nor any of their employees, makes any warranty, express or implied, or assumes any legal liability or responsibility for the accuracy, completeness, or usefulness of any information, apparatus, product, or process disclosed, or represents that its use would not infringe privately owned rights. Reference herein to any specific commercial product, process, or service by trade name, trademark, manufacturer, or otherwise, does not necessarily constitute or imply its endorsement, recommendation, or favoring by the United States Government or the University of California. The views and opinions of authors expressed herein do not necessarily state or reflect those of the United States Government or the University of California, and shall not be used for advertising or product endorsement purposes.

This research was supported under the auspices of the U.S. Department of Energy by the University of California, Lawrence Livermore National Laboratory under contract No. W-7405-Eng-48.

This report has been reproduced directly from the best available copy.

Available electronically at http://www.doe.gov/bridge

Available for a processing fee to U.S. Department of Energy

and its contractors in paper from

U.S. Department of Energy

Office of Scientific and Technical Information

P.O. Box 62

Oak Ridge, TN 37831-0062

Telephone: (865) 576-8401

Facsimile: (865) 576-5728

E-mail: reports@adonis.osti.gov

Available for the sale to the public from

U.S. Department of Commerce

National Technical Information Service

5285 Port Royal Road

Springfield, VA 22161

Telephone: (800) 553-6847

Facsimile: (703) 605-6900

E-mail: orders@ntis.fedworld.gov

OR

Lawrence Livermore National Laboratory

Technical Information Departments Digital Library

http://www.llnl.gov/tid/Library.html

Approved for public release; further dissemination unlimited 


\title{
An Object Oriented, Finite Element Framework For Linear Wave Equations
}

\author{
by \\ JOSEPH MATTHEW KONING \\ B.A. (University of California at Berkeley) 1992 \\ M.S. (San Jose State University) 1997 \\ DISSERTATION \\ Submitted in partial satisfaction of the requirements for the degree of \\ DOCTOR OF PHILOSOPHY \\ in \\ Engineering - Applied Science \\ in the \\ OFFICE OF GRADUATE STUDIES \\ of the \\ UNIVERSITY OF CALIFORNIA \\ DAVIS
}

Approved:

Chair

Committee in Charge

2004 
An Object Oriented, Finite Element Framework For Linear Wave

Equations

Copyright 2004

by

Joseph Matthew Koning 
Joseph Matthew Koning

January 2004

Engineering - Applied Science

An Object Oriented, Finite Element Framework For Linear Wave Equations

\begin{abstract}
This dissertation documents an object oriented framework which can be used to solve any linear wave equation. The linear wave equations are expressed in the differential forms language. This differential forms expression allows a strict discrete interpretation of the system. The framework is implemented using the Galerkin Finite Element Method to define the discrete differential forms and operators. Finite element basis functions including standard scalar Nodal and vector Nedelec basis functions are used to implement the discrete differential forms resulting in a mixed finite element system. Discretizations of scalar and vector wave equations in the time and frequency domains will be demonstrated in both differential forms and vector calculi. This framework conserves energy, maintains physical continuity, is valid on unstructured grids, conditionally stable and second order accurate. Examples including linear electrodynamics, acoustics, elasticity and magnetohydrodynamics are demonstrated.
\end{abstract}

Professor Garry H. Rodrigue Ph.D. Dissertation Committee Chair 
For Patti, Max, Madeline, Claire and my Family. 


\section{Contents}

List of Figures viii

List of Tables $\quad$ xi

1 Introduction $\quad \mathbf{1}$

1.1 Organization ....................... 7

2 Wave Equations $\quad 10$

2.1 Acoustic Scalar Wave Equation . . . . . . . . . . . . . . . . . . 10

2.2 Electrodynamic Vector Wave Equation . . . . . . . . . . . . . . . . . 14

2.3 Linear Acoustic Vector Wave Equation . . . . . . . . . . . . . . . . . 18

2.4 Linear Elastic Vector Wave Equation . . . . . . . . . . . . . . . . . . 18

2.5 Linear Magnetohydrodynamics Vector Wave Equation . . . . . . . . . 20

2.6 Differential Forms . . . . . . . . . . . . . . . . . . . . . . . . 23

2.6.1 Manifolds ..................... . . 24

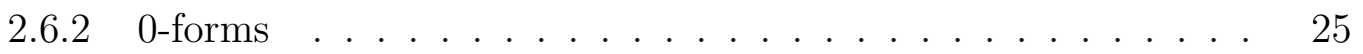

2.6 .3 1-forms . . . . . . . . . . . . . . 26

2.6.4 Exterior product . . . . . . . . . . . . . . . 28

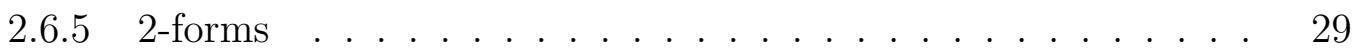

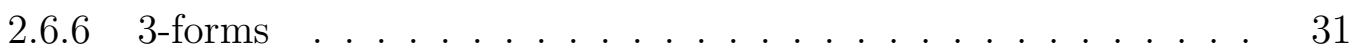

2.6.7 Hodge star operator . . . . . . . . . . . . . . . . . 32

2.6.8 Pushforward and Pullback operators . . . . . . . . . . . 33

2.6.9 Exterior derivative . . . . . . . . . . . . . . . . . . . . . 35

2.6.10 Initial Boundary Value Problem . . . . . . . . . . . . . . . . . 38

2.7 Acoustic Scalar Wave Equation . . . . . . . . . . . . . . . . . . . 40

2.8 Electrodynamic Vector Wave Equation . . . . . . . . . . . . . . . . . 42

2.9 Linear Acoustic Vector Wave Equation . . . . . . . . . . . . . . . . . 43

2.10 Linear Elastic Vector Wave Equation . . . . . . . . . . . . . . . . . . 43

2.11 Linear Magnetohydrodynamics Vector Wave Equation . . . . . . . . . 44

3 Discrete Differential Forms $\quad 45$

3.1 Galerkin Finite Element Method . . . . . . . . . . . . . . . . . 46

3.2 Vector Spaces . . . . . . . . . . . . . . . . . . . . . . . . . . . . . . . . . . . . . . .

3.3 Finite Elements . . . . . . . . . . . . . . . . 50 
3.3 .1 Degrees of freedom . . . . . . . . . . . . . . . . . 52

3.3.2 Linear tetrahedral basis functions . . . . . . . . . . . . . . 53

3.3.3 Linear prismatic basis functions . . . . . . . . . . . . 60

3.3.4 Linear hexahedral basis functions . . . . . . . . . . . . . 66

3.3.5 Bilinear forms . . . . . . . . . . . . . . . . . . . . 73

3.3.6 Discrete differential operators . . . . . . . . . . . . . 74

3.3.7 Basis transformations . . . . . . . . . . . . . . . . . . 78

3.4 Properties of the Discrete $p$-forms $\ldots \ldots \ldots \ldots$

3.5 Spurious Modes . . . . . . . . . . . . . . . . . . . . . . 82

3.6 Examples . . . . . . . . . . . . . . . . . . . . . . . . . . . . . . 84

3.6 .1 Scalar wave equations $\ldots \ldots \ldots \ldots$. . . . . . . . . . 84

3.6 .2 Electromagnetic wave equations . . . . . . . . . . . 88

3.6.3 Linear acoustic vector wave equations . . . . . . . . . . . . . 91

3.6.4 Linear elastic wave equations . . . . . . . . . . . . . . 94

3.6.5 Linear magnetohydrodynamic wave equations . . . . . . . . 94

3.6.6 Second order linear magnetohydrodynamics . . . . . . . . . . 99

4 Linear Algebraic System Analysis 101

4.1 Iterative Methods . . . . . . . . . . . . . . . . . . . . . . . . . 101

4.2 Differential Operator Stencils . . . . . . . . . . . . . 105

4.2.1 Discrete Differential Operators . . . . . . . . . . . . . 107

$4.2 .2 \quad$ 0-form operators . . . . . . . . . . . . . . . . . . . . . . . . 109

4.2 .3 1-form operators . . . . . . . . . . . . . . 111

4.2 .4 2-form operators . . . . . . . . . . . . . . . . 114

4.2 .5 3-form operators . . . . . . . . . . . . . . . . . 117

4.3 Mass Matrix Solution Scaling . . . . . . . . . . . . . . . 117

5 Numerical Method Analysis $\quad 125$

5.1 Scalar Wave Equation . . . . . . . . . . . . . . . . . 127

$5.1 .1 \quad$ Numerical stability . . . . . . . . . . . . . . . . . . . . . 127

$5.1 .2 \quad$ Numerical dispersion . . . . . . . . . . . . . . . . . . . 133

5.1 .3 Energy conservation . . . . . . . . . . . . . . . . . 137

5.2 Electrodynamic Wave Equation . . . . . . . . . . . . . . . . . . . 139

$5.2 .1 \quad$ Numerical stability . . . . . . . . . . . . . . . . . . . . . . . 139

5.2 .2 Numerical dispersion . . . . . . . . . . . . . . . . . . . . . 141

5.2 .3 Magnetic charge conservation . . . . . . . . . . . . . . 145

5.2 .4 Electric charge conservation . . . . . . . . . . . . . . 146

5.2.5 Energy conservation . . . . . . . . . . . . . . . . . . 149

5.3 Linear Acoustic Vector Wave Equation . . . . . . . . . . . . . . 150

5.3 .1 Numerical stability . . . . . . . . . . . . . . . . . . 150

5.3 .2 Numerical dispersion . . . . . . . . . . . . . . . . . . . . . 152

5.3 .3 Vorticity conservation 1 -forms . . . . . . . . . . . . 155

5.3 .4 Vorticity conservation 2-forms . . . . . . . . . . . 157

5.3.5 Energy conservation . . . . . . . . . . . . . . . . . . 159

5.4 Linear Elastic Wave Equation . . . . . . . . . . . . . . . . 160

5.4 .1 Numerical stability . . . . . . . . . . . . . . . . . . 160 
5.4 .2 Numerical dispersion . . . . . . . . . . . . . . . . . . . 160

5.5 Linear Magnetohydrodynamic Wave Equation . . . . . . . . . . . . 161

5.5.1 Numerical stability . . . . . . . . . . . . . . . . . . . 161

5.5.2 Numerical dispersion . . . . . . . . . . . . . . . . . . . 162

5.5.3 Energy conservation . . . . . . . . . . . . . . 163

6 Parallel Implementation $\quad 165$

6.1 Parallel Toolkits . . . . . . . . . . . . . . . . 166

6.2 Python . . . . . . . . . . . . . . . . . . . . . 167

6.3 Mesh Generation and Domain Decomposition . . . . . . . . . . . . 168

$\begin{array}{lll}7 \text { Validation } & 171\end{array}$

7.1 Common simulation meshes . . . . . . . . . . . . . . . . . . . . 172

7.2 Operator conformance . . . . . . . . . . . . . . . . 174

7.3 Scalar Wave Equation . . . . . . . . . . . . . . . . . . 176

7.3.1 Space characterization . . . . . . . . . . . . . . 176

7.3.2 Spherical cavity . . . . . . . . . . . . . . . . . . 180

7.4 Electrodynamic Vector Wave Equation . . . . . . . . . . . . . . . . 187

7.4.1 Space characterization . . . . . . . . . . . . . . . 187

7.4.2 Spherical cavity . . . . . . . . . . . . . . . . . . . 191

7.5 Acoustic Vector Wave Equation . . . . . . . . . . . . . . . . . . . . 198

7.5.1 Space characterization . . . . . . . . . . . . . . . 198

7.5.2 Spherical cavity . . . . . . . . . . . . . . . . 201

7.6 Combined Vector Wave Equations . . . . . . . . . . . . . . . . . . 209

7.6.1 Linear elastic waves . . . . . . . . . . . . . . . . . 209

7.6.2 Linear magnetohydrodynamic modes . . . . . . . . . . . . . . 212

7.7 Parallel Results . . . . . . . . . . . . . . . . . . . . 214

8 Results 219

8.1 Optical fiber waveguide . . . . . . . . . . . . . . . . . . 219

8.2 Photonic band gap waveguide . . . . . . . . . . . . . . . . . . . . . . . . . . . . . . . . . 222

8.3 Sonic band gap waveguide . . . . . . . . . . . . . . . 231

9 Conclusions 235

$\begin{array}{ll}\text { Bibliography } & 237\end{array}$

A Reduced Unit Formulation for Electrodynamics 242

A.0.1 Second Order Reduced Wave Equation . . . . . . . . . . . . . 242

A.0.2 First Order Reduced Wave Equation . . . . . . . . . . . . . . 244

B Vector Identities $\quad 245$ 


\section{List of Figures}

2.1 Graphical description of a 0-form where the quantity shown is the evaluation of the scalar function at $(0,3,2) \ldots \ldots . . \ldots 26$

2.2 Graphical description of a 1 -form $d x^{2} \ldots \ldots$. . . . . . . . . . . . 27

2.3 Graphical description of a 1-form $d x^{2}$ integrated from $(1,0,0)$ to $(1,3,0) .27$

2.4 Graphical description of a 2 -form $d x^{1} \wedge d x^{2}$. . . . . . . . . . . . . 30

2.5 Graphical description of a 2-form $d x^{1} \wedge d x^{2}$ integrated over a plane indicated by the dotted line segments. . . . . . . . . . . . . . 30

2.6 Graphical description of a 3-form $d x^{1} \wedge d x^{2} \wedge d x^{3}$ integrated over the

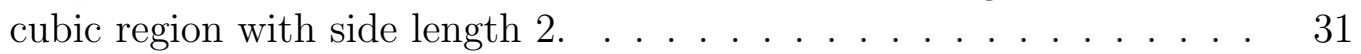

2.7 Pullback Operation. . . . . . . . . . . . . . . . . . . . 34

2.8 Push-Forward Operation. . . . . . . . . . . . . . . . . . . 35

3.1 Tetrahedron Basis Function Numbering . . . . . . . . . . . . . . 54

3.2 Tetrahedron Node Basis Functions . . . . . . . . . . . . . . . 55

3.3 Tetrahedron Edge Basis Functions . . . . . . . . . . . . . . . 57

3.4 Tetrahedron Face Basis Functions . . . . . . . . . . . . . . . . 58

3.5 Tetrahedron Volume Basis Functions . . . . . . . . . . . . . . . 59

3.6 Prism Basis Function Numbering . . . . . . . . . . . . . . . . 60

3.7 Prism Node Basis Functions . . . . . . . . . . . . . . . . . . . 62

3.8 Prism Edge Basis Functions . . . . . . . . . . . . . . . 63

3.9 Prism Face Basis Functions . . . . . . . . . . . . . . . 65

3.10 Prism Volume Basis Functions . . . . . . . . . . . . . . . 66

3.11 Hexahedral Basis Function Numbering . . . . . . . . . . . . . . 67

3.12 Hexahedral Node Basis Functions . . . . . . . . . . . . . . . . . . . 68

3.13 Hexahedral Edge Basis Functions . . . . . . . . . . . . . . . . 70

3.14 Hexahedral Face Basis Functions . . . . . . . . . . . . . . . . . . . . 72

3.15 Hexahedral Volume Basis Functions . . . . . . . . . . . . . . 73

4.1 Scalar 2-Dimensional Laplacian . . . . . . . . . . . . . . . . . . . . 106

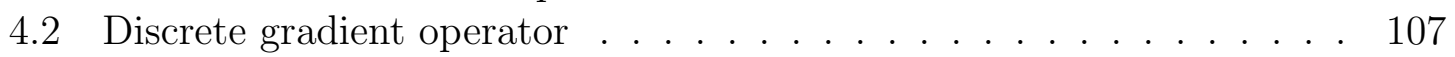

4.3 Discrete curl operator . . . . . . . . . . . . . . . . . . . . . . 108

4.4 Discrete divergence operator . . . . . . . . . . . . . . . . . . . 108

4.5 0-form DivGrad operator . . . . . . . . . . . . . . . . . . . . . . . . 109

4.6 0-form DivGrad Operator with Lumped Stiffness Matrix . . . . . . . 110 
4.7 1-form Curl-Curl Operators . . . . . . . . . . . . . . . 111

4.8 1-form Curl-Curl Operator with Lumped Stiffness Matrix . . . . . . . 112

4.9 1-form Adjoint Grad-Div Operator . . . . . . . . . . . . . . . 113

4.10 1-form Laplacian . . . . . . . . . . . . . . . . . . . . . . . . . . . . . 114

4.11 2-form Grad-Div Operator . . . . . . . . . . . . . . . . . . 115

4.12 2-form Adjoint Curl-Curl Operator . . . . . . . . . . . . . . 115

4.13 2-form Laplacian . . . . . . . . . . . . . . . . . . . . . . . 116

4.14 3-form DivGrad operator . . . . . . . . . . . . . . . . . 117

4.15 2D Quadrilateral Meshes . . . . . . . . . . . . . . . . 119

4.16 2D Quadrilateral Refinement Meshes . . . . . . . . . . . . . 123

5.1 Second order natural Div-Grad solutions . . . . . . . . . . . . . . . . 132

5.2 Second order adjoint Div-Grad solutions . . . . . . . . . . . . . . . 134

5.3 Second order natural Curl-Curl solutions . . . . . . . . . . . . . . . . 140

5.4 Second order adjoint Curl-Curl solutions . . . . . . . . . . . . . . . . 142

5.5 Discrete Curl for Highlighted Edge in a Tetrahedron . . . . . . . . . . 145

5.6 Second order natural Grad-Div solutions . . . . . . . . . . . . . . . 151

5.7 Second order adjoint Grad-Div solutions . . . . . . . . . . . . . . . 153

5.8 Discrete Gradient for Highlighted Node in a Tetrahedron . . . . . . . 156

5.92 Dimensional MHD term Stencil . . . . . . . . . . . . . . . . . . 162

6.1 Matrix Partitioning . . . . . . . . . . . . . . 167

6.2 Cylinder Mesh . . . . . . . . . . . . . . . . . . . . . . . 169

6.3 Cylinder Mesh Domain Decomposition . . . . . . . . . . . . . . . . 170

7.1 Power Spectra for the 0-form acoustic simulation for the series of hexahedral grids $\left(\frac{h}{a}=\frac{1}{4}, \frac{1}{6}, \frac{1}{8}, \frac{1}{10}\right.$; left to right,top to bottom) . . . . . . . . 182

7.2 Power Spectra for the 0 -form acoustic simulation for the series of tetrahedral grids $\left(\frac{h}{a}=\frac{1}{4}, \frac{1}{6}, \frac{1}{8}, \frac{1}{10}\right.$; left to right,top to bottom $) \ldots . . . . . .183$

7.3 Mesh Refinement Results for 0-form Pressure Wave Equation . . . . . 184

7.4 Power Spectra for the 3-form acoustic simulation for the series of hexahedral grids $\left(\frac{h}{a}=\frac{1}{4}, \frac{1}{6}, \frac{1}{8}, \frac{1}{10}\right.$; left to right,top to bottom $)$. . . . . . . 185

7.5 Power Spectra for the 3-form acoustic simulation for the series of tetrahedral grids $\left(\frac{h}{a}=\frac{1}{4}, \frac{1}{6}, \frac{1}{8}, \frac{1}{10}\right.$; left to right,top to bottom $) \ldots 186$

7.6 Mesh Refinement Results for 3-form Density Wave Equation . . . . . 188

7.7 Power Spectra for the 1-form electrodynamic simulation for the series of hexahedral grids $\left(\frac{h}{a}=\frac{1}{4}, \frac{1}{6}, \frac{1}{8}, \frac{1}{10}\right.$;left to right,top to bottom) . . . . 193

7.8 Power Spectra for the 1-form electrodynamic simulation for the series of tetrahedral grids $\left(\frac{h}{a}=\frac{1}{4}, \frac{1}{6}, \frac{1}{8}, \frac{1}{10}\right.$;left to right,top to bottom) . . . . 194

7.9 Mesh Refinement Results for 1-form Electric Field Wave Equation . . 195

7.10 Power Spectra for the 2-form electrodynamic simulation for the series of hexahedral grids $\left(\frac{h}{a}=\frac{1}{4}, \frac{1}{6}, \frac{1}{8}, \frac{1}{10}\right.$; left to right,top to bottom) . . . . 196

7.11 Power Spectra for the 2-form electrodynamic simulation for the series of tetrahedral grids $\left(\frac{h}{a}=\frac{1}{4}, \frac{1}{6}, \frac{1}{8}, \frac{1}{10}\right.$;left to right,top to bottom) . . . . 197

7.12 Mesh Refinement Results for 2-form Magnetic Field Wave Equation . 198

7.13 Power Spectra for the 1-form acoustic simulation for the series of hexahedral grids $\left(\frac{h}{a}=\frac{1}{4}, \frac{1}{6}, \frac{1}{8}, \frac{1}{10}\right.$; left to right,top to bottom $) \ldots . .$. 
7.14 Power Spectra for the 1-form acoustic simulation for the series of tetrahedral grids $\left(\frac{h}{a}=\frac{1}{4}, \frac{1}{6}, \frac{1}{8}, \frac{1}{10}\right.$; left to right, top to bottom) . . . . . . 204

7.15 Mesh Refinement Results for 1-form Velocity Field Wave Equation . . 205

7.16 Power Spectra for the 2-form acoustic simulation for the series of hexahedral grids $\left(\frac{h}{a}=\frac{1}{4}, \frac{1}{6}, \frac{1}{8}, \frac{1}{10}\right.$; left to right,top to bottom) . . . . . 206

7.17 Power Spectra for the 2-form acoustic simulation for the series of tetrahedral grids $\left(\frac{h}{a}=\frac{1}{4}, \frac{1}{6}, \frac{1}{8}, \frac{1}{10}\right.$; left to right,top to bottom) . . . . . 207

7.18 Mesh Refinement Results for 2-form Velocity Field Wave Equation . . 208

7.19 2D 2-form linear elastic wave displacement magnitude . . . . . . . 211

7.202 Dimensional Acoustic and MHD Term Eigenmodes . . . . . . . . 213

7.21 Hexahedral Grid Jacobi CG Run Times . . . . . . . . . . . . . . . . 217

7.22 Hexahedral Grid Jacobi CG Speedup . . . . . . . . . . . . . . 218

8.1 Bent optical fiber grid. . . . . . . . . . . . . . . . . . . . 223

8.2 TE01 pulse propagating down a straight optical fiber. . . . . . . . . 224

8.3 TE01 pulse propagating down an optical fiber with a 30 degree bend. 225

8.4 TE01 pulse propagating down an optical fiber with a 45 degree bend. 226

8.5 TE01 pulse propagating down an optical fiber with a 60 degree bend. 227

8.6 2D photonic band gap mesh. . . . . . . . . . . . . . . . . . 229

8.7 2D Photonic band gap waveguide electric field magnitude. . . . . . 230

8.8 2D sonic band gap mesh. . . . . . . . . . . . . . . . . . 233

8.9 2D Sonic band gap waveguide velocity magnitude. . . . . . . . . . 234 


\section{List of Tables}

1.1 Properties of the $p$-forms f. . . . . . . . . . . . . 2

2.1 Exterior Operations . . . . . . . . . . . . . . . . . . . . . 29

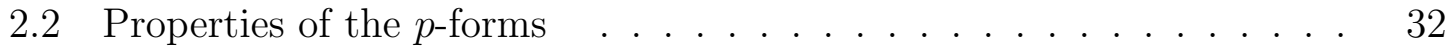

2.3 Transformation Rules . . . . . . . . . . . . . . . . . . . 33

2.4 Natural and Adjoint Differential Operators . . . . . . . . . . . . . . . 38

2.5 Generalized Stoke's Law . . . . . . . . . . . . . . . . . 38

3.1 Degrees of Freedom . . . . . . . . . . . . . . . . . . 53

3.2 Tetrahedral Basis Functions . . . . . . . . . . . . . . . 54

3.3 Prismatic Basis Functions . . . . . . . . . . . . . . . 61

3.4 Hexahedral Basis Functions . . . . . . . . . . . . . . . . . 67

3.5 Discrete Differential Operators . . . . . . . . . . . . . . . . 75

3.6 Properties of the $p$-forms . . . . . . . . . . . . . . . 81

4.1 Two dimensional edge basis functions for a Quadrilateral . . . . . . . 120

4.2 Condition number of 2D edge element mass matrix . . . . . . . . . . 120

4.3 Condition number of 2D edge element diagonally preconditioned system 121

4.4 Number of conjugate gradient iterations for $f=0.44 \ldots$. . . . . . 121

4.5 Ratio of maximum to minimum zone area for various grid sizes . . . . 122

4.6 Ratio of maximum to minimum edge length for various grid sizes . . 122

4.7 Mesh Metrics for refined mesh sequence $f=0.35$. . . . . . . . . 123

4.8 Condition number for mass matrix and diagonally preconditioned mass matrix for refined mesh sequence $f=0.35$. . . . . . . . . . . . . . . 124

4.9 Condition number for diagonally scaled conj. grad. and ILU conj.

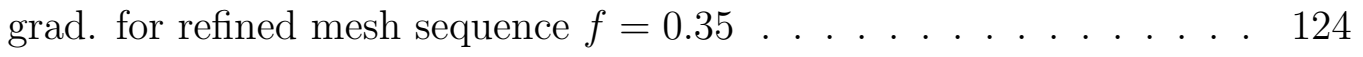

5.1 Statistics for the hexahedral sphere grid. . . . . . . . . . . . . . . 131

5.2 Space dimensions for the natural Div-Grad operator test equations on a hexahedral sphere grid. . . . . . . . . . . . . . . . . . . . . . . 132

5.3 Space dimensions for the adjoint Div-Grad operator test equations on a hexahedral sphere grid . . . . . . . . . . . . . . . . . . . . . . . 133

5.4 Nodal Dispersion Degrees Of Freedom . . . . . . . . . . . . . . 135

5.5 Space dimensions for the natural Curl-Curl operator test equations on a hexahedral sphere grid. . . . . . . . . . . . . . . . . . . . 140 
5.6 Space dimensions for the adjoint Curl-Curl operator test equations on a hexahedral sphere grid . . . . . . . . . . . . . . . . . . . . . . 141

5.7 Edge Dispersion Degrees Of Freedom . . . . . . . . . . . . . . . 143

5.8 Space dimensions for the natural Grad-Div operator test equations on a hexahedral sphere grid. . . . . . . . . . . . . . . . . . . . . 151

5.9 Space dimensions for the adjoint Grad-Div operator test equations on a hexahedral sphere grid . . . . . . . . . . . . . . . . . . 152

5.10 Face Dispersion Degrees Of Freedom . . . . . . . . . . . . . 154

7.1 Rectangular Cavity Meshes _. . . . . . . . . . . . . . . . . . . . 172

7.2 Spherical Cavity Hexahedral Refinement Meshes . . . . . . . . . . . 173

7.3 Spherical Cavity Tetrahedral Refinement Meshes . . . . . . . . . . 173

7.4 Cylindrical Cavity Meshes . . . . . . . . . . . . . . . . . . . 173

7.5 Rectangular Mesh Vector Operator Conformance . . . . . . . . . . 175

7.6 Cylindrical Mesh Vector Operator Conformance . . . . . . . . . . . 175

7.7 Rectangular Mesh Stiffness Matrix Conformance . . . . . . . . . . 175

7.8 Cylindrical Mesh Stiffness Matrix Conformance . . . . . . . . . . 176

7.9 Div-Grad Eigenvalues with Dirichlet Boundary Conditions . . . . . . 178

7.10 Div-Grad Discrete Space Dimensions for Dirichlet Boundary Conditions . . . . . . . . . . . . . . . . . . . . 178

7.11 Div-Grad Eigenvalues with Neumann Boundary Conditions . . . . . 179

7.12 Div-Grad Discrete Space Dimensions for Neumann Boundary Conditions . . . . . . . . . . . . . . . . . . . . . . . . 179

7.13 Exact Lowest Acoustic Frequencies . . . . . . . . . . . . . . . 181

7.14 0-form Acoustic Refinement Results . . . . . . . . . . . . . . . 182

7.15 3-form Acoustic Refinement Results . . . . . . . . . . . . . . . 187

7.16 Curl-Curl Eigenvalues with Dirichlet Boundary Conditions . . . . . . 189

7.17 Curl-Curl Discrete Space Dimensions for Dirichlet Boundary Conditions . . . . . . . . . . . . . . . . . . . . . . . . 189

7.18 Curl-Curl Eigenvalues with Neumann Boundary Conditions . . . . . 190

7.19 Curl-Curl Discrete Space Dimensions for Neumann Boundary Conditions . . . . . . . . . . . . . . . . . . . . 190

7.20 Exact Lowest Electrodynamic Frequencies _ . . . . . . . . . . . . 192

7.21 1-form Electrodynamic Refinement Results . . . . . . . . . . . 195

7.22 2-form Electrodynamic Refinement Results . . . . . . . . . . . 198

7.23 Grad-Div Eigenvalues with Dirichlet Boundary Conditions . . . . . . 200

7.24 Grad-Div Discrete Space Dimensions for Dirichlet Boundary Conditions . . . . . . . . . . . . . . . . . 200

7.25 Grad-Div Eigenvalues with Neumann Boundary Conditions . . . . . 201

7.26 Grad-Div Discrete Space Dimensions for Neumann Boundary Conditions . . . . . . . . . . . . . . . . . . . . 201

7.27 1-form Acoustic Refinement Results . . . . . . . . . . . . . . . 205

7.28 2-form Acoustic Refinement Results . . . . . . . . . . . . . . . 208

7.29 Vector Laplacian Eigenvalues with Dirichlet Boundary Conditions . . 210

7.30 Hexahedral Elastic Wave Mesh . . . . . . . . . . . . . . . . 211

7.312 2-form Elastic wave speeds . . . . . . . . . . . . . . . . . 212 
7.32 MHD term eigenvalues . . . . . . . . . . . . . . . . . . . . 212

7.33 MHD term eigenvalues . . . . . . . . . . . . . . . . . 213

7.34 Hexahedral Grid Parallel Results . . . . . . . . . . . . . . 215

8.1 Bend radii for the bent optical fibers . . . . . . . . . . . . . . 221

8.2 Optical fiber parameters . . . . . . . . . . . . . . . . . 222

8.3 Curvature loss for the bent optical fibers . . . . . . . . . . . . 222 


\section{Acknowledgements}

I wish to acknowledge my parents for having the good sense of having a fifth and final child thus achieving perfection.

I would also like to thank my technical adviser Dr. Daniel White for imparting his immense knowledge upon me and possessing the patience to do so.

Prof. Garry Rodrigue has guiding me through my graduate career and given me some insight into the bizarre worlds of Academia and its relation to large government labs. He also makes a fine Cabernet Sauvignon, not too hot or loaded with coconut.

This dissertation would not be complete without the help of Dr. Paul Castillo and Mark Stowell their contribution came in the form of time-saving high quality code that is beyond price to a time-constrained graduate student.

The dissertation is typeset in $\mathrm{IAT}_{\mathrm{E}} \mathrm{X}$ and uses many other free and open source products, I wish to thank all of the authors for their contribution.

The staff in the Department of Applied Science have been very helpful making my graduate life smooth and pleasant.

Lawrence Livermore National Laboratory supported me financially through the Student Employee Graduate Research Fellowship program, without this aid I, most likely, would not have pursued a Ph.D..

Last, but certainly not least I wish to thank my wife Patricia for putting up with me through two degrees. This is above and beyond what any person should have to endure. For all the times you have taken up the slack when I couldn't be there I hope to repay you in double. Now it is your turn to lie on the couch and eat bon-bons. Max,Madeline and Baby X will now, hopefully, have a full time father. 


\section{Chapter 1}

\section{Introduction}

It is the aim of this dissertation to develop a framework based on differential forms and the finite element method that correctly models linear wave equations so that all of the physical quantities inherent in the equations are conserved. A discrete method that models energy conservation as well as material interface continuity and specific physical quantity conservation (i.e. charge) is said to be mimetic in that it mimics the physics of the equation. This is accomplished by utilizing the concepts of the differential forms calculus on a discrete level. The discrete differential forms are constructed via the finite element method such that they maintain all of the properties of the continuous differential forms. Conservation properties inherent in the continuous differential forms are maintained in the discrete level, providing a provably conservative method. This dissertation uses a discrete representation of the differential forms to create a method for translating continuous linear wave equations into their discrete representations. The differential forms equation representation allows a direct interpretation into discrete objects such as physical quantity representations 
and differential operators that define the object oriented nature of the framework.

The specific partial differential equations studied in this dissertation are wave equations. The wave equations are scalar or vector hyperbolic partial differential equations describing the transport of field information in time and space. Two and three-dimensional, linear wave equations in the fields of acoustics, electrodynamics, elasticity, and magnetohydrodynamics will be presented in vector calculus and differential forms for generality. For each of the wave equations listed, a discrete wave equation and simulations showing the stability and conservation of the method will be presented. Some of these discretizations have been developed previously. What this dissertation provides is a new interpretation of these discretizations and new discretizations based on the framework.

Differential forms [1] are covariant tensors. In a practical view they are four unique entities in three dimensional space integrated over a point, line, surface or volume resulting in a number. The collection of differential forms are referred to as $p$-forms where the $p=0,1,2,3$ in $p$-forms refers to the rank of the covariant tensor. Each of the $p$-forms has specific properties with respect to its continuity, derivative, and integral as shown in Table 1.1. The various $p$-forms will be discussed in more

Table 1.1: Properties of the $p$-forms

\begin{tabular}{|c|c|c|c|c|}
\hline Property & 0-form & 1-form & 2-form & 3-form \\
\hline \hline Minimum Continuity & Total & Tangential & Normal & None \\
\hline Integral & Point & Line & Surface & Volume \\
\hline Derivative & Grad & Div & Curl & None \\
\hline
\end{tabular}

detail in Chapter 2.

The Galerkin finite element method will be used to construct a discrete differential 
forms framework. Four specific basis functions are introduced that conform with the continuous differential forms properties in Table 1.1. One or more of the four basis functions can be utilized in the linear wave equations. Using more than one basis function in the discretization is referred to as the mixed finite element method [2]. This dissertation will explain the construction and analysis of a discrete differential forms based mixed finite element framework. To construct the framework properties of the discrete differential forms must mimic the continuous differential forms. These properties include:

- Discrete spaces that mimic continuous spaces.

- Discrete differential operators that mimic continuous operators.

- Metric free discrete differential operators.

- Discrete spaces and differential operators that form exact sequences.

- Automatic conservation of energy, divergence free and curl free fields.

- Correct continuity of fields across material interfaces.

The exact sequence defines a series of differential operators that map a differential $p$-form into the next higher $p$-form for $p=0,1,2$. If this exact sequence can be maintained on a discrete level the method can be shown to be conservative. The construction of the differential operators maintains the physical continuity of the physical fields by creating discrete differential forms which lie in spaces that are subspaces of the continuous differential forms. If the previous requirements are met, then a field that is initially divergence or curl free will remain divergence or curl free. 
In addition to these properties, the discrete differential forms framework will have the following properties:

- Elimination of spurious modes.

- Well defined on structured and unstructured tessellations.

- Synergy between discrete simulation code for different differential forms.

- Second order accurate in space and time.

- Conditionally stable.

- Allows for scalar and tensor materials with spatial discontinuities.

Spurious modes arise in discrete simulations where the continuity, divergence or curl of a field are not maintained correctly. If these properties are not maintained then the discrete system is incorrectly constructed resulting in unphysical solutions. Using the finite element based discrete differential forms framework eliminates these spurious modes, allows discrete systems based on unstructured grids, and provides a means to reuse simulation code across the various wave equations.

In recent years discrete differential forms methods, the finite element version of mimetic methods, have gained popularity. These methods are based on the idea that discrete operators should come from the discrete version of the continuous space, maintaining all of the conservation properties. Ensuring the relation of the discrete spaces to the continuous spaces allows the creation of differential operators that are physically accurate, thus leading to methods that conserve all of the physical properties. 
Research has been performed on finite difference, finite volume, and finite element discrete differential forms methods [3]. All three methods have been previously developed without a differential forms interpretation and are currently being reinterpreted in terms of discrete differential forms.

The definitive mimetic finite difference method for electromangetics is the Finite Difference Time Domain (FDTD) method. Since the method was introduced by Yee in 1966 [4] the goal has been to generalize this method to non-orthogonal and unstructured grids. The methods listed below all reduce to FDTD for orthogonal structured grids. It is the goal of this dissertation to define operators on unstructured grids for all of the wave equations listed above that reduce to the corresponding finite difference operators on structured orthogonal grids.

In the early 1980's Samarskii, Tishkin, Favorskii and Shashkov [5] [6] [7] developed the finite difference discrete differential forms method referred to as the Support Operator Method (SOM). This method forms natural and adjoint differential operators [8], [9] that form an exact sequence. One limitation of the SOM is the restriction to non-orthogonal but structured meshes with well defined dual-grids.

Around the same time the support operator method was being developed, Nédélec [10] [11] was developing vector finite element basis functions that conform to the mininmum continuity of 1 -forms 2 -forms. The discrete 1 -forms and 2 -forms can be totally continuous but must also maintain tangential and normal continiuty,repectively, across material interfaces. Standard totally continuous nodal and discontinuous volume centered scalar basis functions coupled with these new vector basis functions define the discrete differential forms finite element basis functions. The 2-form or face basis 
functions are the three-dimensional extension of the two-dimensional finite elements used by Raviart and Thomas [12]. While the finite-element based discrete differential forms method is mainly being used in the field of electrodynamics [13], [14], researchers have been actively analyzing the underlying mathematics [15]. The finite element method based on these basis functions maintains the exact sequence and continuity properties described in the SOM.

The finite integration technique (FIT) is a conservative finite volume method developed in 1977 by Weiland [16] for Maxwell's equations. This technique uses the integral form of Maxwell's equations to form the discrete differential operators that form an exact sequence. Continuity of the various $p$-forms is also maintained as in the previous two methods. In a recent paper [17], the FIT is recast in terms of discrete differential forms where the Curl-Curl operator is shown to be symmetric. Previous attempts to define a conservative finite volume method resulted in non-symmetric second order Curl-Curl operators [18] [19] [20] [21]. This non-symmetric Curl-Curl operator leads to late-time instabilities [22] for electrodynamic simulations in the time-domain. To counteract these instabilities, dissipative integration schemes may be used, but these lead to violation of conservation of energy and charge [23].

The main focus of the research demonstrated in this dissertation is the simulation of optical devices such as step-index optical fibers and photonic band gap devices. Performing full wave, finite element, time-domain simulations of optical fibers requires large numbers of elements. This in turn requires a parallel framework. Photonic band gap devices are devices with complicated periodic structures that are modeled more appropriately by conforming meshes. The development of a code to model these 
devices lead to a more general method that allowed for simulations of all of the wave equations listed above. Comparisons of known solutions for the various wave equations will be performed as well as simulations for complicated optical devices with no known solution.

This dissertation develops a general discrete differential forms finite element framework for simulating wave equations in the time and frequency domains. The method will be shown to be second order accurate in space and time, provable stable and conservative, and valid on unstructured meshes. The wave equations can be solved in natural or adjoint form while preserving divergence-free or curl-free field constraints automatically. The second order spatial operators are combinations of a natural and an adjoint first order operator. In this dissertation the terms natural and adjoint will refer to the first operator used in the second order spatial operator. Simulations for linear electrodynamics, linear acoustics, linear elasticity, and magnetohydrodynamics will be presented.

\section{$1.1 \quad$ Organization}

Chapter 2 begins the dissertation with an introduction to Differential Forms and the equivalent Vector Calculus forms of various wave equations. These initial boundary value problems (IBVP) will be discussed as examples of a general IBVP presented in differential forms.

Chapter 3 shows derivation of the discrete differential forms framework in which all of the wave equations are cast. It will be shown that the discrete differential forms framework preserves the conservation of all physical quantities as well as automatically 
enforcing divergence-free or curl-free field constraints. This is accomplished through the use of four basis functions corresponding to the four forms. These four basis functions mimic the properties of continuous and discontinuous scalar fields as well as tangential and normally continuous vector fields. To solve the wave equations using a finite element method, they must be placed in variational form. Using the variational forms and discrete differential forms, the discrete linear system of equations will be derived.

Chapter 4 defines the linear algebraic method used to solve the sparse linear systems resulting from the finite element discretization. For each of the operators utilized in the wave equations, equivalent finite difference stencils are presented with their corresponding accuracy. The scalability of the linear system solution method for the two-dimensional electrodynamic wave equation is also discussed.

Chapter 5 presents the analysis of the numerical methods used in the wave equation simulations. Included in the discussion are results for analyses of stability, dispersion, and conservation of energy, charge, and vorticity.

Chapter 6 discusses the parallel implementation used in the simulations. The wave equation simulations are all based on the same time-stepping and linear system solution code. This code only differs in the mass and stiffness matrices and boundary conditions. The framework is object-oriented implemented in c++ [24] and python [25] and runs in parallel on three different operating systems and nine different architectures. The linear system solution and matrix-vector algebra routines are implemented through the use of PETSc, the portable, extensible toolkit for scientific computation [26] [27] [28]. 
Chapter 7 validates the method using frequency and time-domain simulations for the wave equations listed in Chapter 2. The accuracy, adaptability, and scalability of the framework are proven through a set of representative problems.

Chapter 8 presents the results of time-domain simulations for large scale simulations and simulations which require conforming unstructured meshes.

Chapter 9 concludes the dissertation with a summary of the object oriented finite element framework for wave equations based on differential forms. 


\section{Chapter 2}

\section{Wave Equations}

This chapter introduces the scalar and vector wave equations used as the example wave equations throughout the dissertation. They will first be represented in vector calculus form to define the variables and equations in a commonly accepted format. Differential forms will then be introduced and used to describe the same equations. This will be done by defining two prototypical initial boundary value problems and defining each wave equation in terms of these prototypes. The specific $p$-form used to describe the physical variable will be chosen based on the continuity requirement of the physical variable.

\subsection{Acoustic Scalar Wave Equation}

In general the scalar wave equation is given by (2.1).

$$
\frac{\partial^{2} \psi}{\partial t^{2}}=\frac{1}{c^{2}} \nabla \cdot \nabla \psi
$$


where $c$ is the wave speed. The simulations presented for the scalar wave equation will be linear acoustic wave equations. The scalar wave equation can be written for the density $\rho$, pressure $P$, or velocity potential $\phi$. The conservation formulas for the situation where the oscillations are so quick that energy is not transfered through heat defining an adiabatic system, are given in (2.2),(2.3), and (2.4) where $\gamma$ is the ratio of specific heats $\gamma=\frac{c_{p}}{c_{v}}$.

$$
\begin{gathered}
\frac{\partial \rho}{\partial t}=-\nabla \cdot(\rho \vec{v}) \\
\frac{\partial(\rho \vec{v})}{\partial t}+\vec{v} \cdot \nabla(\rho \vec{v})=-\nabla(P) \\
P=K \rho^{\gamma}
\end{gathered}
$$

To linearize the scalar acoustic wave equations, a small perturbation is introduced around a constant mean value for density (2.5) and pressure (2.6). The velocity is considered to be a perturbation alone with no mean value (2.7).

$$
\begin{gathered}
\rho(\vec{r}, t)=\rho_{0}(\vec{r})+\rho_{1}(\vec{r}, t) \\
P(\vec{r}, t)=P_{0}(\vec{r})+P_{1}(\vec{r}, t) \\
\vec{v}(\vec{r}, t)=\vec{v}_{1}(\vec{r}, t)
\end{gathered}
$$

Combining (2.5),(2.6), and (2.7) with (2.8),(2.9), and (2.10) gives the linear conservation equations for linear acoustics.

$$
\begin{gathered}
\frac{\partial \rho_{1}}{\partial t}=-\rho_{0} \nabla \cdot \vec{v}_{1} \\
\rho_{0} \frac{\partial\left(\vec{v}_{1}\right)}{\partial t}=-\nabla\left(P_{1}\right) \\
P_{1}=\frac{P_{0} \gamma}{\rho_{0}} \rho_{1}
\end{gathered}
$$


The first order acoustic wave equations are usually written as functions of the pressure (2.11) and velocity (2.12).

$$
\begin{aligned}
& \frac{\partial P_{1}}{\partial t}=-\left(\gamma P_{0}\right) \nabla \cdot v_{1} \\
& \frac{\partial v_{1}}{\partial t}=-\frac{1}{\rho_{0}} \nabla P_{1}
\end{aligned}
$$

Taking the partial time derivative of the first equation for pressure and substituting into the first order velocity equation leads to the second-order wave equations for density (2.13) and pressure (2.14)

$$
\begin{aligned}
& \frac{\partial^{2} \rho_{1}}{\partial t^{2}}=c_{l}^{2} \nabla \cdot \nabla \rho_{1} \\
& \frac{\partial^{2} P_{1}}{\partial t^{2}}=c_{l}^{2} \nabla \cdot \nabla P_{1}
\end{aligned}
$$

where $c_{l}^{2}=\frac{P_{0} \gamma}{\rho_{0}}$ is the sound speed. The velocity potential $\phi(2.15)$ can be introduced if the initial distribution of velocities is irrotational $(\nabla \times \vec{v}=0)[29]$ resulting in the scalar wave equation for the velocity potential (2.16).

$$
\begin{gathered}
\vec{v}=-\nabla \phi \\
\frac{\partial^{2} \phi}{\partial t^{2}}=c_{l}^{2} \nabla \cdot \nabla \phi
\end{gathered}
$$

In the density scalar wave equation (2.13), the density $\rho_{1}$ is usually represented as a discontinuous scalar function. The boundary conditions for this equation are given in (2.17), where $D$ is the scalar value of the density on the boundary and $N$ is the scalar value of normal gradient of the density on the boundary.

$$
\begin{aligned}
\rho_{1} & =D \text { on } \Gamma_{d} \\
\nabla \rho_{1} \cdot \hat{n} & =N \text { on } \Gamma_{n}
\end{aligned}
$$


To define a well-posed problem for the second-order wave equation an initial condition and the partial derivative in time of the initial condition are required (2.18). In these equations the density variable can be replaced by pressure or the velocity potential.

$$
\begin{aligned}
\rho_{1}(0, t) & =\rho_{i c} \\
\frac{\partial \rho_{1}(0, t)}{\partial t} & =\frac{\partial \rho_{i c}}{\partial t}
\end{aligned}
$$

In the pressure scalar wave equation (2.14), the pressure $P$ is usually represented as a continuous scalar function. The boundary conditions for this equation are given in (2.19), where $D$ is the scalar value of the pressure on the boundary and $N$ is the scalar value of the normal gradient of the pressure on the boundary.

$$
\begin{aligned}
P_{1} & =D \text { on } \Gamma_{d} \\
\nabla P_{1} \cdot \hat{n} & =N \text { on } \Gamma_{n}
\end{aligned}
$$

In some simulations in this dissertation the wave equation will be analyzed in the frequency domain. This is accomplished by separating the quantity (density, pressure or velocity potential) into a spatial and time part e.g. $\rho_{1}=\psi(\vec{r}) T(t)$. For the wave equations, the time part $T(t)$ is harmonic and can be represented by $T(t)=e^{-i \omega t}$ where $\omega$ is the frequency of oscillation. The linear scalar acoustic wave equation in the frequency domain after substituting the harmonic time function is given in (2.20) where the dispersion relation is $k=\frac{\omega}{c_{l}}$ and the spatial part $\psi(\vec{r})$ can be for the density, pressure or velocity potential.

$$
\nabla \cdot \nabla \psi-k^{2} \psi=0
$$

The linear acoustic wave equation is a non-dissipative system, therefore it will satisfy energy conservation. The wave intensity of the acoustics in units $\left[\frac{W}{m^{2}}\right]$ is given 
by the Poynting vector $\vec{S}=P_{1} \vec{v}_{1}$. Looking at the divergence of this quantity gives $(2.21)$

$$
\begin{aligned}
\nabla \cdot\left(P_{1} \vec{v}_{1}\right) & =\vec{v}_{1} \cdot \nabla P_{1}+P_{1} \nabla \cdot \vec{v}_{1} \\
\nabla \cdot \vec{S} & =-\rho_{0} \vec{v}_{1} \cdot \frac{\partial \vec{v}_{1}}{\partial t}-\left(\gamma P_{0}\right)^{-1} P_{1} \frac{\partial P_{1}}{\partial t} \\
\nabla \cdot \vec{S} & =-\frac{1}{2} \frac{\partial}{\partial t}\left[\rho_{o}\left|\vec{v}_{1}^{2}\right|+\left(\gamma P_{0}\right)^{-1} P_{1}^{2}\right]
\end{aligned}
$$

The power is given by $(2.22)$ where the kinetic energy density is $\epsilon_{k i n}=\frac{1}{2} \rho_{0}\left|{\overrightarrow{v_{1}}}^{2}\right|$ and the potential energy density is $\epsilon_{p o t}=\frac{1}{2} \frac{P_{1}^{2}}{\gamma P_{0}}$.

$$
\oint_{\Gamma} \vec{S} \cdot \hat{n} d \Gamma+\frac{1}{2} \frac{\partial}{\partial t} \int_{\Omega}\left[\rho_{o}\left|\vec{v}_{1}^{2}\right|+\left(\gamma P_{0}\right)^{-1} P_{1}^{2}\right]=0
$$

\subsection{Electrodynamic Vector Wave Equation}

In their most general form Maxwell's equations (2.23), contain the electric $\vec{E}$ and magnetic fields $\vec{H}$ as well as the total electric current density $\vec{J}_{\text {tot }}$ and total magnetic current density $\vec{M}_{t o t}$. Maxwell's equations also include the conservation

of electric and magnetic charge which are comprised of the electric flux density $\vec{D}$, magnetic flux density $\vec{B}$, electric charge density $\rho_{e c}$, and magnetic charge density $\rho_{m c}$. The electric and magnetic fields are represented by vector fields with a continuous tangential component of the field equal across material boundaries. This is referred to as tangential continuity [30]. The electric and magnetic flux densities and currents are represented by vector fields which have the normal component of the field equal across material boundaries. The flux densities maintain normal continuity across a material interface [30]. The charge densities are represented by a quantity that can 
be discontinuous between regions of space.

$$
\begin{aligned}
\nabla \times \vec{E} & =-\vec{M}_{t o t} \\
\nabla \times \vec{H} & =\vec{J}_{t o t} \\
\nabla \cdot \vec{D} & =\rho_{e c} \\
\nabla \cdot \vec{B} & =\rho_{m c}
\end{aligned}
$$

The total electric current density can be decomposed into three components $(2.24)$ : a source current density $\vec{J}_{s}$, the conduction current density $\vec{J}_{c}$, and the displacement current density $\overrightarrow{J_{d}}$. These electric current densities all have units of amperes per square meter.

$$
\begin{aligned}
\vec{J}_{t o t} & =\vec{J}_{s}+\vec{J}_{x}+\vec{J}_{d} \\
\vec{J}_{d} & =\frac{\partial \vec{D}}{\partial t}
\end{aligned}
$$

The total magnetic current density can also be decomposed into three components (2.25): a source current density $\vec{M}_{s}$, a fictitious "conduction" current density $\vec{M}_{c}$, and the displacement current density $\vec{M}_{d}$.

$$
\begin{aligned}
\vec{M}_{t o t} & =\vec{M}_{s}+\vec{M}_{x}+\vec{M}_{d} \\
\vec{M}_{d} & =\frac{\partial \vec{B}}{\partial t}
\end{aligned}
$$

This dissertation will analyze only the linear Maxwell's equations in charge free regions. This leads to linear relations between the electric and magnetic fields and their corresponding flux densities. These linear relations are given in (2.26), where $\epsilon, \mu, \sigma_{E}$ and $\sigma_{M}$ are tensor quantities corresponding to the electric permittivity, mag- 
netic permeability, electric conductivity, and magnetic conductivity respectively.

$$
\begin{aligned}
\vec{D} & =\epsilon \vec{E} \\
\vec{B} & =\mu \vec{H} \\
\vec{J}_{c} & =\sigma_{E} \vec{E} \\
\vec{M}_{c} & =\sigma_{M} \vec{H}
\end{aligned}
$$

Combining the current densities and material relations with the general Maxwell's equations (2.23) gives the first-order Maxwell's equations (2.27).

$$
\begin{aligned}
\frac{\partial \vec{B}}{\partial t} & =-\nabla \times \vec{E}-\vec{M}_{s}-\sigma_{M} \mu^{-1} \vec{B} \\
\frac{\partial \epsilon \vec{E}}{\partial t} & =\nabla \times \mu^{-1} \vec{B}-\vec{J}_{s}-\sigma_{E} \vec{E} \\
\nabla \cdot \epsilon \vec{E} & =0 \\
\nabla \cdot \vec{B} & =0 \\
\hat{n} \times \vec{E} & =\vec{E}_{\Gamma} \text { on } \Gamma \\
\vec{E}(0, t) & =\vec{E}_{i c} \\
\vec{B}(0, t) & =\vec{B}_{i c}
\end{aligned}
$$

The second order vector wave equation for the electric field in an inhomogeneous charge free domain is constructed by taking the partial derivative of the second equation and substituting into the first equation in (2.27) giving (2.28).

$$
\begin{aligned}
\frac{\partial^{2} \epsilon \vec{E}}{\partial t^{2}}+\left(\sigma_{E}+\mu^{-1} \sigma_{M} \epsilon\right) \frac{\partial \vec{E}}{\partial t}+\mu^{-1} \sigma_{M} \sigma_{E} \vec{E}= & -\nabla \times \mu^{-1} \nabla \times \vec{E}-\mu^{-1} \sigma_{M} \vec{J}_{s} \\
& -\mu^{-1} \nabla \times \vec{M}_{s}-\frac{\partial \vec{J}_{s}}{\partial t} \\
\nabla \cdot \epsilon \vec{E} & =0 \\
\hat{n} \times \vec{E} & =\vec{E}_{\Gamma} \text { on } \Gamma
\end{aligned}
$$




$$
\begin{aligned}
\vec{E}(0, t) & =\vec{E}_{i c} \\
\frac{\partial \vec{E}(0, t)}{\partial t} & =\frac{\partial \vec{E}_{i c}}{\partial t}
\end{aligned}
$$

The second order vector wave equation can also be written for the magnetic flux density in an inhomogeneous charge free domain (2.29).

$$
\begin{aligned}
\frac{\partial^{2} \vec{B}}{\partial t^{2}}+\left(\sigma_{M} \mu^{-1}-\epsilon^{-1} \sigma_{E}\right) \frac{\partial \vec{B}}{\partial t}+\epsilon^{-1} \sigma_{E} \sigma_{M} \mu^{-1} \vec{B} & =-\nabla \times \epsilon^{-1} \nabla \times \mu^{-1} \vec{B} \\
& -\epsilon^{-1} \nabla \times \vec{J}_{s}-\epsilon^{-1} \sigma_{E} \vec{M}_{s} \\
\nabla \cdot \vec{B} & =0 \\
\hat{n} \cdot \vec{B} & =\vec{B}_{\Gamma} \quad \text { on } \quad \Gamma \\
\vec{B}(0, t) & =\vec{B}_{i c} \\
\frac{\partial \vec{B}(0, t)}{\partial t} & =\frac{\partial \vec{B}_{i c}}{\partial t}
\end{aligned}
$$

The Maxwell's equations define a Poynting vector as in the acoustic equations. In this case the power flow is perpendicular to the electric and magnetic fields. The divergence of the Poynting vector $\vec{S}=\vec{E} \times \mu^{-1} \vec{B}$ integrated over all space gives the integral form of the conservation of energy for Maxwell's equations (2.30).

$$
\begin{array}{r}
\int_{\Omega} \nabla \cdot \vec{S} d \Omega=\oint_{\Gamma}\left(\vec{E} \times \mu^{-1} \vec{B}\right) \cdot \hat{n} d \Gamma+\int_{\Omega} \vec{E} \cdot \vec{J}_{s}+ \\
\int_{\Omega} \mu^{-1} \vec{B} \cdot \vec{M}_{s}+\int_{\Omega} \epsilon \vec{E} \cdot \frac{\partial \vec{E}}{\partial t}+\int_{\Omega} \mu^{-1} \vec{B} \cdot \frac{\partial \vec{B}}{\partial t}+ \\
\int_{\Omega} \sigma_{E} \vec{E} \cdot \vec{E}+\int_{\Omega} \mu^{-1} \sigma_{B} \vec{B} \cdot \vec{B}=0
\end{array}
$$




\subsection{Linear Acoustic Vector Wave Equation}

The section on scalar wave equations presents the acoustic wave equations for pressure, density and vector potential. Combining the first-order equations for velocity instead of the scalar pressure gives the second-order acoustic wave equation for velocity (2.31).

$$
\frac{\partial^{2} \vec{v}}{\partial t^{2}}=c_{l}^{2} \nabla(\nabla \cdot \vec{v})
$$

For the acoustic wave equations, the velocity field is constrained to be irrotational $\nabla \times \vec{v}=0$. The tangential component of the velocity field at the boundary can be non-zero even if the normal component is zero. The boundary conditions enforced for this equation are the normal component of the velocity at the boundary and the divergence of the velocity, the pressure, at the boundary (2.32).

$$
\begin{aligned}
& \vec{v} \cdot \hat{n}=\vec{D} \text { on } \Gamma_{d} \\
& \nabla \cdot \vec{v}=N \text { on } \Gamma_{n}
\end{aligned}
$$

The conservation of energy for this equation is the same as for the scalar case. The vector acoustic wave equation is another representation of the scalar acoustic wave equation.

\subsection{Linear Elastic Vector Wave Equation}

The partial differential equation that describes the linearized displacement field [31], [32], [33] is given by (2.33).

$$
\rho \frac{\partial^{2} \vec{u}}{\partial t^{2}}=\nabla \cdot \overline{\bar{\tau}}+\rho \vec{f}
$$


This equation is the conservation of linear and angular momentum where the density, $\rho$ is constant. The quantity $\overline{\bar{\tau}}$ is the combined stress and strain tensors given by $(2.34)$

$$
\overline{\bar{\tau}}=2 \mu \overline{\bar{\epsilon}}+\lambda \operatorname{Tr}(\overline{\bar{\epsilon}})
$$

where the linear stress tensor $\overline{\bar{\epsilon}}$ is given in $(2.35)$ and $\operatorname{Tr}(\overline{\bar{\epsilon}})$ is the trace of the linear stress tensor. The two constants $\lambda$ and $\mu$ are called the Lamé constants. The constant $\mu$ is the measure of a body's resistance to shear strain and is called the shear modulus. The constant $\lambda$ does not have a simple physical interpretation. The longitudinal or sound speed $c_{l}$ in a rigid body is given in terms of the Lamé constants by (2.36). The linear elasticity equations also support transverse waves, which introduce a transverse wave speed $c_{t}$ given in terms of the Lamé constants by (2.37).

$$
\begin{gathered}
\overline{\bar{\epsilon}}=\frac{1}{2}\left(\nabla \vec{u}+(\nabla \vec{u})^{T}\right) \\
c_{l}^{2}=\frac{(\lambda+2 \mu)}{\rho} \\
c_{t}^{2}=\frac{\mu}{\rho}
\end{gathered}
$$

Combining equations $(2.33),(2.34),(2.36)$, and (2.37) forms the second-order linear elastic wave equation for displacement (2.38). The operator on the right hand side corresponds to the vector Laplacian operator.

$$
\frac{\partial^{2} \vec{u}}{\partial t^{2}}=c_{l}^{2} \nabla(\nabla \cdot \vec{u})-c_{t}^{2} \nabla \times \nabla \times \vec{u}
$$

The boundary conditions for an elastic wave equation specify the traction (2.39) and the displacement (2.40).

$$
\begin{gathered}
\vec{t}=\overline{\bar{\epsilon}} \cdot \hat{n} \text { on } \Gamma \\
\vec{u}=\vec{D} \text { on } \Gamma
\end{gathered}
$$


The Poynting theorem for linear elastic waves involves the combined stress and strain tensors $\tau$. In the linear acoustic equations this tensor reduces to $\nabla P_{1}$ for inviscid flows. The energy can be derived from the the displacement equation (2.33) by dotting this equation with the velocity $\vec{v}=\frac{\partial \vec{u}}{\partial t}$, resulting in the differential form of the energy equation (2.41) with no external forces applied.

$$
\rho \frac{\partial^{2} \vec{u}}{\partial t^{2}} \cdot \frac{\partial \vec{u}}{\partial t}=\nabla \cdot\left(\overline{\bar{\tau}} \cdot \frac{\partial \vec{u}}{\partial t}\right)
$$

Using the identity (B.4) the energy equation for linear elasticity (2.42) can be constructed.

$$
\nabla \cdot(\vec{S}) \equiv \nabla \cdot-(\overline{\bar{\tau}} \cdot \vec{v})=\frac{1}{2} \frac{\partial}{\partial t}\left\{\rho \frac{\partial \vec{u}}{\partial t} \cdot \frac{\partial \vec{u}}{\partial t}+\overline{\bar{\tau}} \cdot \overline{\bar{\epsilon}}\right\}
$$

Just as in the acoustic and electrodynamics equations there is an energy flux term on the right hand side. The first and second terms on the left-hand side contain the kinetic and potential energy density terms respectively. In integral form the energy equation becomes (2.43).

$$
\oint_{\Gamma}-(\overline{\bar{\tau}} \cdot \vec{v}) \cdot \hat{n} d \Gamma=\frac{1}{2} \frac{\partial}{\partial t} \int_{\Omega}\left\{\rho \frac{\partial \vec{u}}{\partial t} \cdot \frac{\partial \vec{u}}{\partial t}+\overline{\bar{\tau}} \cdot \overline{\bar{\epsilon}}\right\} d \Omega
$$

\subsection{Linear Magnetohydrodynamics Vector Wave Equation}

The linear magnetohydrodynamics (MHD) equations mark a departure from the standard differential forms description of the wave equations above. The purpose behind examining the MHD equations is to show the extensible nature of a differential 
forms framework. These equations are derived from the nonlinear magnetohydrodynamics equations by solving for a small perturbations around a steady state just as in the acoustic wave equations. The magnetic flux density equation as well as Ohm's law for a charged fluid in motion are given in (2.44) and (2.45) respectively.

$$
\begin{gathered}
\frac{\partial \vec{B}}{\partial t}=\nabla \times(\vec{v} \times \vec{B})+\frac{1}{\mu \sigma} \nabla^{2} \vec{B} \\
\vec{J}=\sigma(\vec{E}+\vec{v} \times \vec{B})
\end{gathered}
$$

The linear magnetohydrodynamics equation is derived under the idealized conditions of a compressible, inviscid, perfectly conducting fluid with no external fields applied other than a constant magnetic flux density. The perfectly conducting nature of the fluid removes the second term in (2.44) and reduces (2.45) to $\vec{E}=-\vec{v} \times \vec{B}$.

The first-order nonlinear magnetohydrodynamics equations under these conditions are given in (2.46).

$$
\begin{aligned}
\frac{\partial \rho}{\partial t} & =-(\nabla \cdot \rho \vec{v}) \\
\rho \frac{\partial \vec{v}}{\partial t} & =-\rho(\vec{v} \cdot \nabla \vec{v})-\nabla P_{1}-\frac{\vec{B}}{\mu} \times \nabla \times \vec{B} \\
\frac{\partial \vec{B}}{\partial t} & =\nabla \times(\vec{v} \times \vec{B})
\end{aligned}
$$

Using (2.5),(2.6),(2.7), and the introduction of the linearized magnetic flux density (2.47) these equations are linearized. The time independent magnetic flux density $\vec{B}_{0}$ is a spatially uniform field.

$$
\vec{B}=\vec{B}_{0}+\vec{B}_{1}(\vec{r}, t)
$$


Substituting the pressure for density as in the acoustic case leads to the first-order magnetohydrodynamics equations (2.48).

$$
\begin{aligned}
\frac{\partial P_{1}}{\partial t} & =-\left(\gamma P_{0}\right)\left(\nabla \cdot \vec{v}_{1}\right) \\
\rho_{0} \frac{\partial \vec{v}_{1}}{\partial t} & =-\nabla P_{1}-\frac{\vec{B}_{0}}{\mu_{0}} \times \nabla \times \vec{B}_{1} \\
\frac{\partial \vec{B}_{1}}{\partial t} & =\nabla \times\left(\vec{v}_{1} \times \vec{B}_{0}\right) \\
\nabla \cdot \vec{B} & =0
\end{aligned}
$$

The choice of boundary conditions is problem dependent, for the simulations in this dissertation the domain will be a closed region so that $\vec{v} \cdot \hat{n}=0$ on $\Gamma$. The domain is embedded in a uniform magnetic flux density $\overrightarrow{B_{0}}$, at the boundaries of this region the magnetic flux density perturbation must be equal to this uniform magnetic flux density $\vec{B}_{1}=\vec{B}_{0}$ on $\Gamma$.

The second-order magnetohydrodynamics equation for velocity is given in (2.49),

$$
\rho_{0} \frac{\partial^{2} \vec{v}_{1}}{\partial t^{2}}=-c_{l}^{2} \nabla \nabla \cdot \vec{v}_{1}-\vec{v}_{a} \times \nabla \times \nabla \times\left(\overrightarrow{v_{a}} \times \vec{v}_{1}\right)
$$

where $\vec{v}_{a}=\sqrt{\frac{\vec{B}_{0}}{\rho_{0}}}$ is the Alfvén velocity.

The magnetic flux density added to the acoustic equations adds to the energy as well. The energy added is the magnetic flux density component shown as the fifth term of (2.30). The magnetohydrodynamics Poynting equation is given in (2.50).

$$
\begin{array}{r}
\nabla \cdot \vec{S}=-\rho_{0} \vec{v}_{1} \cdot \frac{\partial \vec{v}_{1}}{\partial t}-\left(\gamma P_{0}\right)^{-1} P_{1} \frac{\partial P_{1}}{\partial t}-\mu^{-1} \vec{B} \cdot \frac{\partial \vec{B}}{\partial t} \\
\nabla \cdot \vec{S}=-\frac{1}{2} \frac{\partial}{\partial t}\left[\rho_{o}\left|\vec{v}_{1}^{2}\right|+\left(\gamma P_{0}\right)^{-1} P_{1}^{2}+\mu^{-1} B^{2}\right] \\
\oint_{\Gamma} \vec{S} \cdot \hat{n} d \Gamma+\frac{1}{2} \frac{\partial}{\partial t} \int_{\Omega}\left[\rho_{o}\left|\vec{v}_{1}^{2}\right|+\left(\gamma P_{0}\right)^{-1} P_{1}^{2}+\mu^{-1} B^{2}\right]=0
\end{array}
$$




\subsection{Differential Forms}

Differential Forms are a subset of a larger subject in mathematics called Geometric Calculus [34]. The main concept of Geometric Calculus is its emphasis on the geometric interpretation of vectors, differential operators and all of the other ideas that combine to form a calculus. Both Differential Forms and Geometric Calculus are reinterpretations of vector calculus for metric free and higher order geometries. These concepts are useful in areas such as spacetime physics but can also be leveraged for use in described linear wave equations. The metric free nature of differential forms allows the construction of differential operators, Grad, Div and Curl that are independent of the coordinate system. The importance of this property will become apparent in the Chapter 3.

The differential forms calculus is based on the concept of four entities called $p$ forms in three-dimensional space. The 0 -form and 3-form are both scalar quantities in curvilinear geometry while the 1 -form and 2-form are vector quantities in curvilinear geometry. The differential form takes a $p$-dimensional vector and gives a number. A more restrictive definition of a $p$-form is an expression that occurs under $p$-fold integrals over domains.

To define a complete framework based on differential forms, various operations are required. These metric free operations include the exterior derivative, $d$, corresponding to the gradient, curl and divergence in vector calculus and the exterior product or wedge operator $\wedge$ corresponding to the dot and cross products in vector calculus. Because coordinate transformations are required the pullback operator, push-forward operator and the hodge star operator $\star_{\alpha}$ will be discussed. More operations are de- 
fined in the differential forms calculus, but this subset is enough to perform all the necessary operations in this dissertation. More information on Differential Forms can be found in the text by Burke [1].

The properties of the 0-form, 1-form, 2-form, 3-form, exterior derivative, exterior product, hodge star operator, and the push-forward and pullback operators are described in the following sections.

\subsubsection{Manifolds}

To discuss differential forms, manifolds and their properties must first be discussed. Manifolds are descriptions of space which may be curved and have complicated topology. Euclidean space, $R^{n}$, the set of $n$-tuples $\left(x^{1}, x^{2}, \ldots, x^{n}\right)$ is a common manifold. Locally manifolds look like Euclidean space and a general manifold is built by creating a set of locally Euclidean regions. On this manifold structures can be created such as vector and tangent spaces. Vector spaces are the set of vectors defined over a manifold and a tangent space is the set of all vectors at a single point three dimensional space. In $R^{3}$ a basis for the vector space of curvilinear coordinates can be defined by the vector $\left\{x^{1}, x^{2}, x^{3}\right\}$. At every point $y \in R^{3}$ a tangent vector, the standard basis for this space is (2.51).

$$
\left\{\frac{\partial}{\partial x^{1}}, \frac{\partial}{\partial x^{2}}, \frac{\partial}{\partial x^{3}}\right\}
$$

A vector field in $R^{3}$ is a mapping from $R^{3}$ to the tangent space (2.52).

$$
T(y)=\beta^{1}(y) \frac{\partial}{\partial x^{1}}+\beta^{2}(y) \frac{\partial}{\partial x^{2}}+\beta^{3}(y) \frac{\partial}{\partial x^{3}}
$$


The values $\beta^{i}(y)$ are the components of the vector usually denoted by the column vector $\left\{\beta^{1}(y), \beta^{2}(y), \beta^{3}(y)\right\}^{T}$. At every point $y \in R^{3}$ a space of cotangent vectors can be defined with basis (2.53).

$$
\left\{d x^{1}, d x^{2}, d x^{3}\right\}
$$

The components of the basis vector (2.53) are called differentials and are dual to the

basis (2.51) shown by the expression $d x^{i} \frac{\partial}{\partial x^{i}}=\delta_{i j}$. The vector space $\Delta V(2.54)$, called the cotangent space, is formed using this basis.

$$
\Delta V=\operatorname{Span}\left\{d x^{1}, d x^{2}, d x^{3}\right\}
$$

\subsubsection{0-forms}

The mathematical representation of a 0 -form $\omega^{0}$ is given in (2.55).

$$
\omega^{0}=\beta(y)
$$

The 0-form takes a zero-dimensional vector, a point, and returns a scalar which corresponds to the evaluation of the scalar function at that point. These entities are useful for describing physical quantities that are continuous across a material interface such as potentials. A 0 -form quantity such as the electric potential $\Phi(\vec{x})$ would be represented as an integral at a point (2.56)

$$
\Phi\left(\vec{x}_{0}\right)=\int_{\text {point }} \Phi(\vec{x})
$$

where the construct $\int_{\text {point }} \Phi(\vec{x})=\int \delta\left(\vec{x}-\vec{x}_{0}\right) \Phi(\vec{x}) d \Omega$ is the evaluation of the function $\Phi(\vec{x})$ at the point $\vec{x}_{0}$.

The basis for a 0 -form is simply the basis for curvilinear coordinates in $R^{3}$ given 
by $\left\{x^{1}, x^{2}, x^{3}\right\}$. The component $\beta$ of the 0 -form is the evaluation of the scalar function at a specific point in space. This is graphically represented in Figure 2.1.

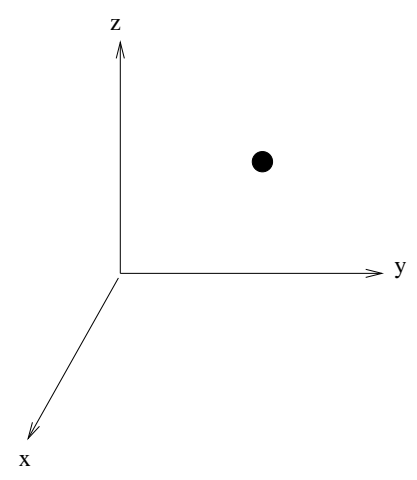

Figure 2.1: Graphical description of a 0-form where the quantity shown is the evaluation of the scalar function at $(0,3,2)$.

\subsubsection{1-forms}

The 1-form is a tensor of rank $\left(\begin{array}{l}0 \\ 1\end{array}\right)$. Tensors of rank one have three elements in three dimensional space. The mathematical representation of a 1 -form $\omega^{1}$ is given in $(2.57)$.

$$
\omega^{1}=\beta_{1}(y) d x^{1}+\beta_{2}(y) d x^{2}+\beta_{3}(y) d x^{3}
$$

The 1-form is a mapping from $R^{3}$ to the cotangent space denoted by $\Delta V$. The basis for the 1-forms is therefore just the cotangent basis (2.53). Each of the components $\beta_{i}$ of the 1-form are 0 -forms.

1-forms correspond to quantities with tangential continuity across a material interface such as the electric field.

As defined above a differential form takes a $p$-dimensional vector and returns a scalar. The scalar that is returned is the result of the integral of the $p$-form over p-dimensional subdomains. In the case of a 1-form this integral is a line integral. A 
graphical representation of the 1-form $d x^{2}$ is shown in Figure 2.2. Only a subset of the entire 1-form $d x^{2}$ is shown in the figure.

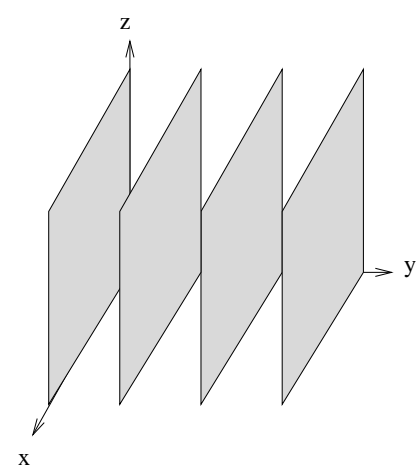

Figure 2.2: Graphical description of a 1-form $d x^{2}$.

The line integral of this form from the point $(1,0,0)$ to $(1,3,0)$ is shown in Figure 2.3. The result of the integral is 4 and is often referred to as placing a pin into an onion, where the pin refers to the line of integration and the layers of the onion are the 1-form.

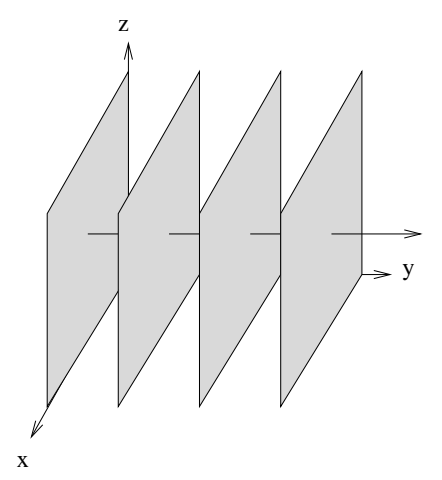

Figure 2.3: Graphical description of a 1-form $d x^{2}$ integrated from $(1,0,0)$ to $(1,3,0)$. 


\subsubsection{Exterior product}

For arbitrary forms $f, g$ and $h$ and scalar values $a$ and $b$, the exterior product, or wedge product $f \wedge g$ is an antisymmetric bilinear operator with the properties listed in $(2.58)$.

$$
\begin{aligned}
(a f+g) \wedge h & =a(f \wedge h)+g \wedge h \\
f \wedge(b h+g) & =b(f \wedge h)+f \wedge g \\
f \wedge f & =0
\end{aligned}
$$

In order to define the 2 -form and 3 -form a wedge product, $\wedge$, must be defined. In the case of the 2-form, the wedge product takes the 1-form basis and returns a 2 -form basis. The result can be thought of as a oriented area and is also referred to as a bivector. The basis for the higher dimensional 2-form cotangent space is given by $(2.59)$.

$$
\left\{d x^{2} \wedge d x^{3}, d x^{3} \wedge d x^{1}, d x^{1} \wedge d x^{2}\right\}
$$

An example of the exterior product of a two 1-forms is shown in (2.60)

$$
\begin{array}{r}
\left(A d x^{1}+B d x^{2}+C d x^{3}\right) \wedge\left(P d x^{1}+Q d x^{2}+R d x^{3}\right)= \\
(B R-C Q) d x^{2} \wedge d x^{3}+(C P-A R) d x^{3} \wedge d x^{1}+(A Q-B P) d x^{1} \wedge d x^{2}
\end{array}
$$

The 3-form cotangent space is formed from the wedge of the 1-form and 2-form cotangent spaces resulting in the 3 -form basis $(2.61)$.

$$
\left\{d x^{1} \wedge d x^{2} \wedge d x^{3}\right\}
$$

An example of the exterior product of a 1-form and a 2-form is shown in (2.62)

$$
\left(A d x^{1}+B d x^{2}+C d x^{3}\right) \wedge\left(P d x^{2} \wedge d x^{3}+Q d x^{3} \wedge d x^{1}+R d x^{1} \wedge d x^{2}\right)=
$$




$$
(A P+B Q+C R) d x^{1} \wedge d x^{2} \wedge d x^{3}
$$

The exterior product corresponds to scalar multiplication, vector dot and cross products in vector calculus. The operator is defined in (2.63) where $\Lambda$ represents the space of an arbitrary form with rank $p, q$ or $p+q$. Table 2.1 lists the exterior product of various combinations of forms and their vector calculus equivalents for scalars $\alpha, \beta$ and vectors $\vec{A}, \vec{B}$.

$$
\wedge: \Lambda^{p} \times \Lambda^{q} \rightarrow \Lambda^{p+q}
$$

Table 2.1: Exterior Operations

\begin{tabular}{|c|c|c|c|}
\hline$p$-form & $q$-form & $(p+q)$-form & Vector Calculus Operation \\
\hline \hline 0 & 0 & 0 & $\alpha \beta$ \\
\hline 0 & 1 & 1 & $\alpha \vec{A}$ \\
\hline 0 & 2 & 2 & $\alpha \vec{B}$ \\
\hline 0 & 3 & 3 & $\alpha \beta$ \\
\hline 1 & 1 & 2 & $\vec{A} \times \vec{B}$ \\
\hline 1 & 2 & 3 & $\vec{A} \cdot \vec{B}$ \\
\hline 2 & 2 & 1 & $\vec{A} \times \vec{B}$ (using Hodge star $)$ \\
\hline
\end{tabular}

The last entry in the table is implemented using the Hodge star operator $\star$ described in the next section and is shown in (2.64). In this identity $\omega, \gamma$ and $\chi$ are 2 -forms and $\delta$ is a 1 -form.

$$
\star(\star \omega \wedge \star \gamma)=\star \delta=\chi
$$

\subsubsection{2-forms}

The mathematical representation of a 2 -form $\omega^{2}$ is given in (2.65).

$$
\omega^{2}=\beta_{1}(y) d x^{2} \wedge d x^{3}+\beta_{2}(y) d x^{3} \wedge d x^{1}+\beta_{3}(y) d x^{1} \wedge d x^{2}
$$


The 2-forms have normal continuity and represent fluxes such as the magnetic flux density.

Graphically 2-forms can be though of as "tubes" such as the intersection of the 1-forms $d x^{1}$ and $d x^{2}$ shown in Figure 2.4. Only a small subset of the entire 2-form is shown in the figure. The integration of the the 2-form is over a 2-dimensional

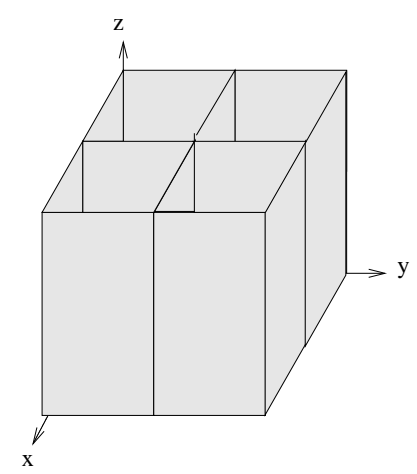

Figure 2.4: Graphical description of a 2-form $d x^{1} \wedge d x^{2}$.

subdomain in this case a plane with normal in the $z$-direction. This plane is denoted by the dotted line in Figure 2.5. The result of the integration is 4 for the four "tubes" intersected by the plane.

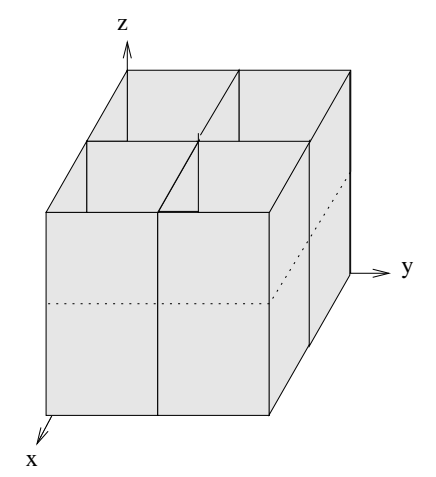

Figure 2.5: Graphical description of a 2-form $d x^{1} \wedge d x^{2}$ integrated over a plane indicated by the dotted line segments. 


\subsubsection{3-forms}

The mathematical representation of a 3 -form $\omega^{3}$ is given in (2.66).

$$
\omega^{3}=\beta(y) d x^{1} \wedge d x^{2} \wedge d x^{3}
$$

Geometrically the value $d x^{1} \wedge d x^{2} \wedge d x^{3}$ is a volume of space. The 3 -forms are defined within a specific volume and therefore have no imposed continuity between adjacent volumes which allows them to represent discontinuous fields such as density.

The graphical description of a 3 -form is shown in Figure 2.6 where it is comprised of "boxes". The integration of a 3-form is over volumes in three-dimensional space. The result of an integration performed on the 3 -form over a volume is the number of boxes contained within the volume. In the case of the 3-form in Figure 2.6 the result is 8 .

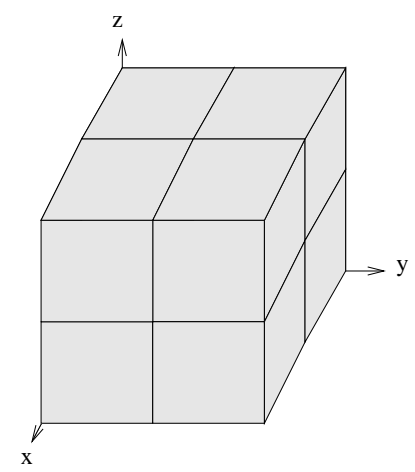

Figure 2.6: Graphical description of a 3-form $d x^{1} \wedge d x^{2} \wedge d x^{3}$ integrated over the cubic region with side length 2 .

In summary the $p$-form properties listed in Table 1.1 can be augmented by the properties listed in the proceeding sections Table 2.2 . 
Table 2.2: Properties of the $p$-forms

\begin{tabular}{|c|c|c|c|c|}
\hline Property & 0-form & 1-form & 2-form & 3-form \\
\hline \hline Minimum Continuity & Total & Tangential & Normal & None \\
\hline Integral & Point & Line & Surface & Volume \\
\hline Derivative & Grad & Div & Curl & None \\
\hline $\begin{array}{c}\text { Physical } \\
\text { Type }\end{array}$ & Potentials & Fields & $\begin{array}{c}\text { Fluxes, } \\
\text { Vector Densities }\end{array}$ & $\begin{array}{c}\text { Scalar } \\
\text { Densities }\end{array}$ \\
\hline Hilbert Space & $\mathcal{H}($ grad $)$ & $\mathcal{H}($ div $)$ & $\mathcal{H}($ curl $)$ & $L^{2}$ \\
\hline
\end{tabular}

\subsubsection{Hodge star operator}

The Hodge star operator is an invertible linear function that maps $p$-forms to (3$p$ )-forms and is defined in (2.67). This operator contains metric and possible material information for a space in $n$ dimensions.

$$
\star: \Lambda^{p} \rightarrow \Lambda^{n-p}
$$

This operator allows the mapping of $p$-forms to $n-p$-forms. The repeated Hodge star operation results in the original form. Examples of the Hodge star operator in a three dimensional Cartesian space are given in (2.68).

$$
\begin{aligned}
\star 1 & =d x^{1} \wedge d x^{2} \wedge d x^{3} \\
\star d x^{1} & =d x^{2} \wedge d x^{3} \\
\star\left(d x^{2} \wedge d x^{3}\right) & =d x^{1} \\
\star\left(d x^{1} \wedge d x^{2} \wedge d x^{3}\right) & =1
\end{aligned}
$$

An example of the Hodge star operator can been seen in the definition of the electric flux density $\vec{D}$. This quantity is defined by $\vec{D}=\epsilon \vec{E}$ in vector calculus. In Differential Forms the electric field is a 1-form quantity possessing tangential continuity across material interfaces. The electric flux density is a 2-form quantity possessing 
normal continuity across material interfaces. The Hodge star operator converts the 1-form electric field to a 2-form electric flux density $D=\star_{\epsilon} E$. Here the Hodge star operator $\star_{\epsilon}$ contains material information via the electric permittivity with units of $[\mathrm{farad} / \mathrm{m}]$. When a Hodge star operator contains material information, the material will be shown as a subscript to the operator.

\subsubsection{Pushforward and Pullback operators}

The push-forward and pullback operators are equivalent to a generalized coordinate transformation in a curvilinear coordinate system. Table 2.3 lists the transformation rules corresponding to the push-forward and pullback for the various $p$-forms . In these transformations $\partial \Phi$ represents the Jacobi transformation matrix in curvilinear coordinates.

Table 2.3: Transformation Rules

\begin{tabular}{|c|c|c|}
\hline$p$-form & Pushforward & Pullback \\
\hline \hline 0 & $(\omega \circ \Phi)$ & $\partial \Phi^{-1}(d \omega \circ \Phi)$ \\
\hline 1 & $\partial \Phi^{-1}(\omega \circ \Phi)$ & $\frac{1}{|\partial \Phi|} \partial \Phi^{T}(d \omega \circ \Phi)$ \\
\hline 2 & $\frac{1}{|\partial \Phi|} \partial \Phi^{T}(\omega \circ \Phi)$ & $\frac{1}{\partial \Phi \mid}(d \omega \circ \Phi)$ \\
\hline
\end{tabular}

Discussion of these transformations can be found in [35], the pictures of the transformations found in this citation are reproduced here in Figure 2.7 and Figure 2.8. The function $f$ maps the manifold $N$ with coordinates $y^{\alpha}$ into the real numbers $f: N \rightarrow R$. The map $\Phi$ can be defined to map the manifold $M$ with coordinates $x^{\mu}$ into $N$ with coordinates $y^{\alpha}, \Phi: M \rightarrow N$. The pullback is then the operation that maps f into $R$ via $M$ instead of $N$ giving $(f \circ \Phi): M \rightarrow R$. The manifolds $M$ and $N$ need not be the same dimension. In this dissertation the manifolds $M$ and $N$ 
correspond to the physical and reference spaces respectively. The mapping $\Phi$ maps positions in the reference space into physical coordinates.

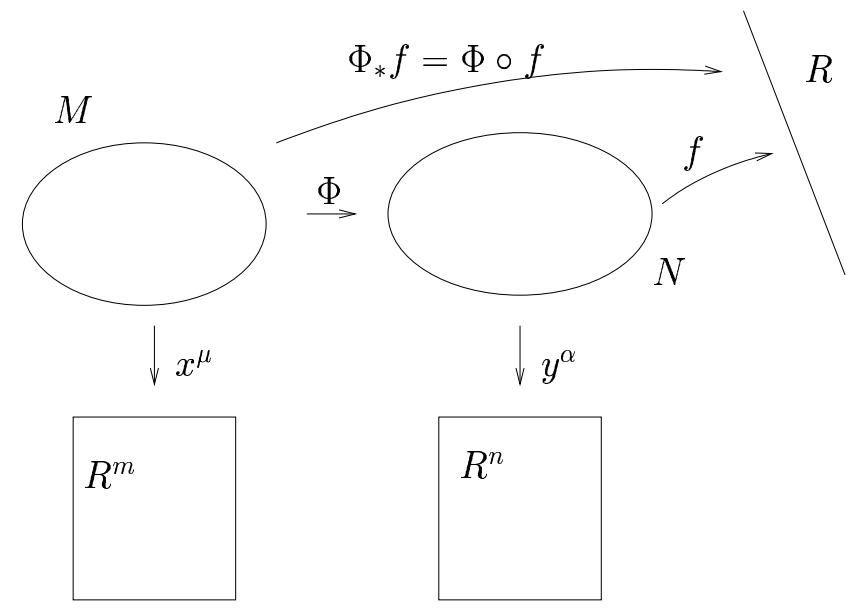

Figure 2.7: Pullback Operation.

An operator cannot be defined using $\Phi$ to create a function in $N$ given a function $g: M \rightarrow R$ in $M$. So a push-forward operator cannot be defined in this way. A vector can be thought of as a derivative operator that maps smooth functions into real numbers [35] . This property allows the definition of a push-forward operator of a vector. If $V(p)$ is a vector at point $p$ in $M$, then the push-forward operator $\Phi_{*}(V(p))$ at a point $\Phi(p)$ on $N$ can be defined by giving its action on functions in $N(2.69)$.

$$
\Phi^{*} V(f)=V\left(\Phi_{*} f\right)
$$

Using the chain rule and the basis for vectors in $M$ given by $\frac{\partial}{\partial x^{\mu}}$ and the basis for vector in $N$ given by $\frac{\partial}{\partial y^{\alpha}}$ the relation between the components of the vector $V=V^{\mu} \frac{\partial}{\partial x^{\mu}}$ in $M$ can be related to the components in $N$ shown in (2.70). This is the generalized version of the vector transformation in vector calculus. The values of $\alpha$ and $\mu$ have different allowed values so the matrix $\frac{\partial y^{\alpha}}{\partial x^{\mu}}$ is not necessarily invertible if $M$ and $N$ are 
different manifolds.

$$
\left(\Phi^{*} V\right)^{\alpha} \frac{\partial f}{\partial y^{\alpha}}=V^{\mu} \frac{\partial y^{\alpha}}{\partial x^{\mu}} \frac{\partial f}{\partial y^{\alpha}}
$$

The push-forward operator is used to transform the forms resulting from an exterior derivative operation. In vector calculus the push-forward operation is the transformation of the vector resulting from a function acting on an initial vector from one coordinate system to another.

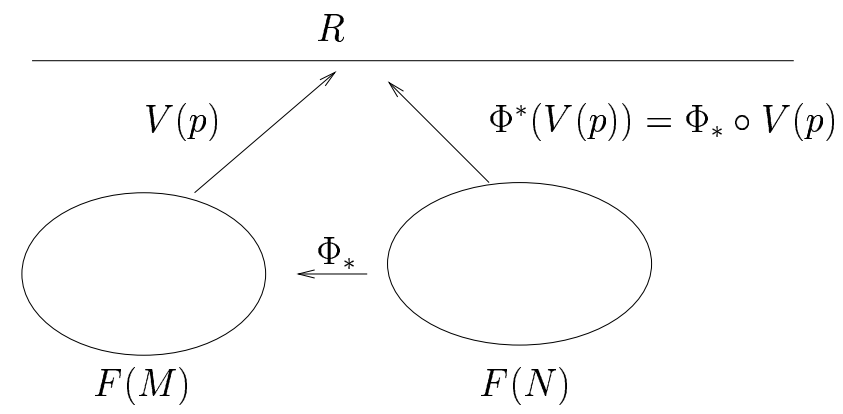

Figure 2.8: Push-Forward Operation.

\subsubsection{Exterior derivative}

Given a 0 -form $f^{0}$, the differential of $f^{0}$ is a 1 -form given by

$$
d \omega^{0}=\frac{\partial \omega^{0}}{\partial x^{1}} d x^{1}+\frac{\partial \omega^{0}}{\partial x^{2}} d x^{2}+\frac{\partial \omega^{0}}{\partial x^{3}} d x^{3}
$$

This operation, referred to as the exterior derivative $d$ defines a linear operator that maps a $p$-form into a $p+1$-form for $p=0,1,2$

$$
d: \Lambda^{p} \rightarrow \Lambda^{p+1} ; \omega \mapsto d \omega
$$

, such that

$$
\begin{aligned}
d\left(f^{l} \wedge g^{m}\right) & =d f^{l} \wedge g^{m}+(-1)^{l m} f^{l} \wedge d g^{m} \\
d\left(d f^{l}\right) & =0
\end{aligned}
$$


The exterior derivative forms an exact sequence between the different forms.

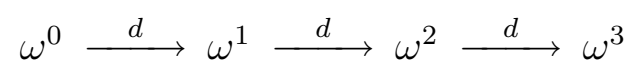

An explicit formula for the exterior derivative of a 1-form can be computed by re-writing a 1-form $\omega^{1}$ as $(2.74)$.

$$
\omega^{1}=A \wedge d x^{1}+B \wedge d x^{2}+C \wedge d x^{3}
$$

Where the components $A, B$, and $C$ are 0 -forms and applying the above chain rule formula to yield (2.75).

$$
\begin{aligned}
d\left(A \wedge d x^{1}+B \wedge d x^{2}+C \wedge d x^{3}\right)= & \left(\frac{\partial C}{\partial x^{2}}-\frac{\partial B}{\partial x^{3}}\right)\left(d x^{2} \wedge d x^{3}\right)+ \\
& \left(\frac{\partial A}{\partial x^{3}}-\frac{\partial C}{\partial x^{1}}\right)\left(d x^{3} \wedge d x^{1}\right)+ \\
& \left(\frac{\partial B}{\partial x^{1}}-\frac{\partial A}{\partial x^{2}}\right)\left(d x^{1} \wedge d x^{2}\right)
\end{aligned}
$$

Likewise, for the exterior derivative of a 2 -form $\omega^{2}$ we have (2.76).

$$
\begin{gathered}
d\left(A \wedge\left(d x^{2} \wedge d x^{3}\right)+B \wedge\left(d x^{3} \wedge d x^{1}\right)+C \wedge\left(d x^{1} \wedge d x^{2}\right)\right)= \\
\left(\frac{\partial A}{\partial x^{1}}+\frac{\partial B}{\partial x^{2}}+\frac{\partial C}{\partial x^{3}}\right)\left(d x^{1} \wedge d x^{2} \wedge d x^{3}\right) .
\end{gathered}
$$

Because the exterior derivative forms an exact sequence, inclusion relations relating the space of the exterior derivative of a form to its resulting form's space can be defined (2.77).

$$
d \omega^{p} \subseteq \omega^{p+1}, p=0,1,2
$$

In vector calculus, the exterior derivatives correspond to the gradient, curl and divergence of the 0-form, 1-form, and 2-form respectively. 


$$
\omega^{0} \stackrel{\nabla}{\longrightarrow} \omega^{1} \stackrel{\nabla \times}{\longrightarrow} \omega^{2} \stackrel{\nabla \cdot}{\longrightarrow} \omega^{3}
$$

The successive operation of the exterior derivative $d d \omega=0$ results in the common vector calculus identities (2.78). These operators are metric free and therefore are exact for any manifold. Examples of the successive exterior derivative in vector calculus are shown in (2.78).

$$
\begin{array}{r}
\nabla \times \nabla \phi=0 \\
\nabla \cdot \nabla \times \vec{E}=0
\end{array}
$$

A set of adjoint differential operators can also be defined for the four forms.

$$
\omega^{0} \stackrel{\star d \star}{\longleftarrow} \omega^{1} \stackrel{\star d \star}{\longleftarrow} \omega^{2} \stackrel{\star d \star}{\longleftarrow} \omega^{3}
$$

Due to the two Hodge star operations these operators are not metric free and in fact include transformations using two metrics corresponding to the two Hodge star operations. The same operations as in (2.78) apply to these adjoint operators.

These adjoint operators map the opposite of the natural operators and are formed using an integration by parts in vector calculus.

$$
{ }^{0} \omega \stackrel{\tilde{\nabla}}{\longleftarrow}{ }^{1} \omega \stackrel{\nabla}{\longleftarrow} \times{ }^{2} \omega \stackrel{\nabla}{\longleftarrow}{ }^{3} \omega
$$


Table 2.4: Natural and Adjoint Differential Operators

\begin{tabular}{|c|c|c|c|c|c|}
\hline \multicolumn{5}{|c|}{ Differential Operators } \\
\hline \hline \multirow{2}{|c|}{} & \multicolumn{4}{c|}{ Domain } \\
\cline { 3 - 6 } \multicolumn{2}{|c|}{ Range } & $\mathcal{H}($ grad,$\Omega)$ & $\mathcal{H}($ curl,$\Omega)$ & $\mathcal{H}($ div,$\Omega)$ & $L^{2}(\Omega)$ \\
\cline { 2 - 6 } & $\mathcal{H}($ curl,$\Omega)$ & & $\tilde{\nabla} \cdot$ & & \\
\cline { 2 - 6 } & $\mathcal{H}($ div,$\Omega)$ & & $\nabla \times$ & $\tilde{\nabla} \times$ & \\
\cline { 2 - 6 } & $L^{2}(\Omega)$ & & & $\nabla \cdot$ & \\
\hline
\end{tabular}

Using the differential operator Stokes law, Gauss's law and the potential law can all be written in a very compact form resulting in the differential forms version of a generalized Stoke's law (2.79).

$$
\int_{\Omega} d \omega^{p}=\int_{\Gamma} \omega^{p}
$$

In this equation $\omega^{p}$ represents a $p$-form, $p=0,1,2, \Omega$ represents a $p+1$ dimensional manifold, and $\Gamma$ represents its boundary.

This compact expression unifies several key integration theorems of vector calculus, shown in Table 2.5 .

Table 2.5: Generalized Stoke's Law

\begin{tabular}{|c|c|c|}
\hline$p=0$ & Fundamental Theorem of Calculus & $\int_{a}^{b} d u=u(b)-u(a)$ \\
\hline$p=1$ & Stokes Theorem & $\int \nabla \times \mathbf{u} \cdot \hat{n} d A=\oint \mathbf{u} \cdot \hat{t} d l$ \\
\hline$p=2$ & Divergence Theorem & $\int \nabla \cdot \mathbf{u} d V=\oint \mathbf{u} \cdot \hat{n} d A$ \\
\hline
\end{tabular}

\subsubsection{Initial Boundary Value Problem}

To define the discrete forms analogs of the previous wave equations, begin with the generic boundary value problem stated in the language of differential forms from [15]. A 3-dimensional domain $\Omega$ with piecewise smooth boundary $\partial \Omega$ partitioned into 
Dirichlet $\Gamma_{D}$, Neumann $\Gamma_{N}$, and Mixed $\Gamma_{M}$ boundary regions is assumed. The problem statement is:

$$
\begin{gathered}
d u=(-1)^{p} \sigma, \quad d j=-\Psi+\Phi \text { in } \Omega \\
T_{D} u=0 \text { on } \Gamma_{D}, T_{N} j=0 \text { on } \Gamma_{N} \\
j=\star_{\alpha} \sigma, \quad \Psi=\star_{\gamma} u \text { in } \Omega \\
T_{M} j=(-1)^{p} \star_{\beta} T_{M} u \text { on } \Gamma_{M} .
\end{gathered}
$$

Here $u$ is a $(p-1)$-form, $\sigma$ is a $p$-form, $j$ is a $(3-p)$-form, and both $\Psi$ and $\Phi$ are $(3-p+1)$-forms, where $1 \leq p \leq 3$. The variable $\Phi$ is a source term. In the boundary conditions (2.81) and (2.83) the symbol $T$ denotes the trace operator, where the trace of a $p$-form is an integral over a $p$-dimensional manifold. Equations (2.80) and (2.82) can be combined to yield the general second-order elliptic equation (2.84).

$$
(-1)^{p} d \star_{\alpha} d u=-\star_{\gamma} u+\Phi .
$$

The adjoint operators, along with the natural operators shown above, will also be used in this paper. The equations associated with the adjoint operators are:

$$
\begin{gathered}
d \sigma=j, \quad d \Psi=-u+\Theta \text { in } \Omega \\
T_{D} u=0 \text { on } \Gamma_{D}, \quad T_{N} \Psi=0 \text { on } \Gamma_{N} \\
\sigma=\star_{\alpha} u, \Psi=\star_{\gamma} j \text { in } \Omega \\
T_{M} \Psi=T_{M} \star_{\beta} u \text { on } \Gamma_{M} .
\end{gathered}
$$

Equations (2.85) and (2.87) can be combined to yield the general second-order adjoint elliptic equation (2.89).

$$
d \star_{\gamma} d \star_{\alpha} u=-u+\Phi .
$$


The second-order natural (2.84) and adjoint (2.89) can be combined to form the Laplacian operator (2.90).

$$
\Delta=d \star_{\gamma} d \star_{\alpha}+(-1)^{p} \star_{\gamma} d \star_{\alpha} d
$$

This operator represents both the scalar and vector Laplacian. The focus of this dissertation is time-dependent phenomena. The time derivative does not affect the degree of a form. The diagram below shows the time-dependent Maxwell's equations, where $d$ denotes the spatial derivative, $d t$ denotes the time derivative and converging arrows denote summation.

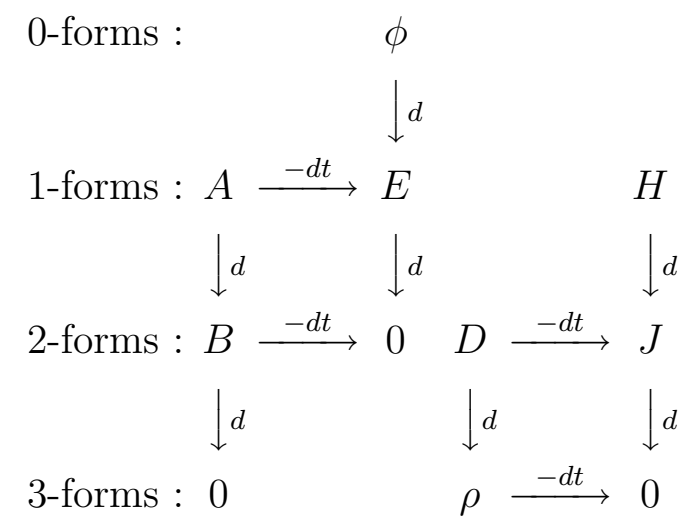

\subsection{Acoustic Scalar Wave Equation}

Now that the more common description of the linear scalar acoustic wave equations have been presented in vector calculus the differential forms version will be described. To describe these PDEs in differential forms first forms and appropriate Hodge star operations must be chosen for each variable. The table listing the various properties of the $p$-forms Table 2.2 makes the choice of a form for each variable quite easy. For the differential forms versions of these equations the equations listed in (2.11) and (2.14) will be discussed. 
To illustrate the choice of differential forms (2.10), (2.11) and (2.12) will be written in integral form shown in (2.91),(2.92), and (2.93) respectively.

$$
\begin{aligned}
\int_{\Omega_{3}} \rho_{1} & =\int_{\Omega_{3}} \frac{\rho_{0}}{\gamma P_{0}} P_{1} \\
\int_{\Omega_{0}} P_{1} & =-\int_{\Omega_{0}} \rho_{0} \nabla \cdot \gamma P_{0} \vec{v}_{1} \\
\int_{\Omega_{1}} \vec{v}_{1} & =-\int_{\Omega_{1}} \nabla P_{1}
\end{aligned}
$$

The natural version of the first-order scalar wave equation with the pressure represented as a 0 -form and the velocity represented as a 1 -form is listed in (2.94).

$$
\begin{aligned}
d_{t} P & =-\star_{\rho_{0}} d \star_{\gamma P_{0}} v \\
d_{t} v & =-d P
\end{aligned}
$$

The operator $d_{t}$ is the differential operator with respect to time instead of space. Combining these two equations by taking the derivative of the first and substituting the second gives the natural second-order form (2.96)

$$
d_{t} d_{t} P=\star_{\rho_{0}} d \star_{\gamma P_{0}} d P
$$

The adjoint first order linear acoustic wave equation (2.11) in differential forms with the pressure represented as a 3-form and the velocity represented as a 2-form is listed in (2.97).

$$
\begin{aligned}
& d_{t} P=-d v
\end{aligned}
$$

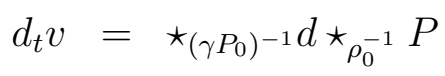

Combining these two equations gives the adjoint second order form (2.99)

$$
d_{t} d_{t} P=d \star_{\left(\gamma P_{0}\right)^{-1}} d \star_{\rho_{0}^{-1}} P
$$




\subsection{Electrodynamic Vector Wave Equation}

In these equations the values of $\star_{\alpha}$ and $\star_{\beta}$ correspond to the material properties $\mu^{-1}$ and $\epsilon$ respectively.

In the differential forms calculus these first order equations become (2.100).

$$
\begin{aligned}
d_{t} B & =-d E-M_{s}-\star_{\sigma_{M}} \star_{\mu^{-1}} B \\
d_{t} D & =d H-J_{s}-\star_{\sigma_{E}} E \\
d D & =0 \\
d B & =0
\end{aligned}
$$

The second order vector wave equation for the electric field in an inhomogeneous charge free domain presented (2.28) has the differential forms description shown in (2.101). This equation represents the natural second order IBVP given in (2.84).

$$
\begin{array}{r}
d_{t} d_{t} D+\left(\star_{\sigma_{E}}+\star_{\mu^{-1}} \star_{\sigma_{M}} \star_{\epsilon}\right) d_{t} E+\star_{\mu^{-1}} \star_{\sigma_{M}} \star_{\sigma_{E}} E \\
=-d \star_{\mu^{-1}} d E-\star_{\mu^{-1}} \star_{\sigma_{M}} J_{s}-d \star_{\mu^{-1}} M_{s}-d_{t} J_{s} \\
d D=0
\end{array}
$$

The second order vector wave equation presented for the magnetic flux density in an inhomogeneous charge free domain in (2.29) has the differential forms representation shown in (2.102). This equation represents the adjoint version of the IBVP given in (2.89).

$$
\begin{array}{r}
d_{t} d_{t} B+\left(\star_{\sigma_{M}} \star_{\mu^{-1}}-\star_{\sigma_{E}} \star_{\epsilon^{-1}}\right) d_{t} B+\star_{\epsilon^{-1}} \star_{\sigma_{E}} \star_{\sigma_{M}} \star_{\mu^{-1}} B= \\
-d \star_{\epsilon^{-1}} d \star_{\mu^{-1}} B-\star_{\epsilon^{-1}} \star_{\sigma_{E}} M_{s}-d \star_{\epsilon^{-1}} J_{s}-d_{t} M_{s} \\
d B=0
\end{array}
$$




\subsection{Linear Acoustic Vector Wave Equation}

The natural and adjoint differential forms descriptions of these equations are the opposite of the second order equations for the scalar case listed in (2.96) and (2.99). The second order linear vector acoustic wave equations for the 2-form natural (2.103) and 1-form adjoint forms (2.105) are constructed from the adjoint first order (2.97) and natural first order (2.94) equations respectively. The boundary conditions presented in (2.32) correspond to representing the velocity as a 2-form corresponding to $(2.84)$.

$$
d_{t} d_{t} v=\star_{\left(\gamma P_{0}\right)^{-1}} d \star_{\rho_{0}^{-1}} d v
$$

If the linear acoustic vector wave equation is written in terms of 1 -forms corresponding to (2.84), the boundary conditions imposed change to (2.104) resulting in (2.105).

$$
\begin{gathered}
\vec{v} \times \hat{n}=\vec{D} \text { on } \Gamma_{d} \\
\vec{v} \cdot \hat{n}=\vec{N} \text { on } \Gamma_{n} \\
d_{t} d_{t} v=d \star_{\rho_{0}} d \star_{\gamma P_{0}} v
\end{gathered}
$$

\subsection{Linear Elastic Vector Wave Equation}

In differential forms the displacement vector $u$ can be describe either by a 1-form or 2-form quantity. In either case the vector Laplacian is a combination of the natural second order and adjoint second order operators. The differential forms versions of (2.38),(2.90), are shown in (2.106) and (2.107) for the 1-form and 2-form versions respectively. 


$$
\begin{aligned}
& d_{t} d_{t} u=d \star_{\rho_{0}^{-1}} d \star_{(\lambda+2 \mu)} u-\star_{\rho_{0}^{-1}} d \star_{\mu} d u \\
& d_{t} d_{t} u=\star_{\rho_{0}^{-1}} d \star_{(\lambda+2 \mu)} d u-d \star_{\rho_{0}^{-1}} d \star_{\mu} u
\end{aligned}
$$

\subsection{Linear Magnetohydrodynamics Vector Wave Equation}

The differential forms version of the first-order linear magnetohydrodynamics equations in (2.48) are listed in (2.108). The velocity $v$, magnetic flux density perturbation $B$ and initial magnetic flux density $B_{0}$ are all described by 2 -forms. The pressure $P$ is described by a 3 -form variable.

$$
\begin{aligned}
d_{t} P & =-d v \\
d_{t} v & =-\star_{\left(\gamma P_{0}\right)^{-1}} d \star_{\rho_{0}^{-1}} P-\star_{\rho_{0}^{-1}} B_{0} \wedge \star_{\rho_{0}^{-1}} d \star_{\mu_{0}^{-1}} B \\
d_{t} B & =d\left(\star v \wedge \star B_{0}\right)
\end{aligned}
$$

Combining the first order differential forms version of the equations into a single second order equation results in the equation listed in (2.109).

$$
d_{t} d_{t} v=\star_{\left(\gamma P_{0}\right)^{-1}} d \star_{\rho_{0}^{-1}} d v-\star_{\rho_{0}^{-1}} B_{0} \wedge \star_{\rho_{0}^{-1}} d \star_{\mu_{0}^{-1}} d\left(\star v \wedge \star B_{0}\right)
$$




\section{Chapter 3}

\section{Discrete Differential Forms}

To define a discrete differential forms framework the discrete differential forms should be a good approximation of the continuous differential forms. Hiptmair [15] outlines the requirements for discrete differential forms. These requirements include: discrete subspaces of the continuous form's space, degrees of freedom that are related to the traces of the forms, conformity to the exact sequence property, and conformity to the commuting diagram. Each of these topics will be discussed in the sections below. The link between differential forms and the finite element basis functions developed by Nédélec [10], [11] leading to the requirements above has been studied by many authors including Bossavit, Mattiussi, Tonti, Dezin, Shashkov, and Chew. The discretization of these differential forms can be accomplished through finite difference, finite volume or finite element techniques as discussed in the introduction. The Galerkin finite element method described in this chapter overcomes two of the major limitations of finite difference and finite volume techniques. These two limitations include the necessity for structured but not necessarily orthogonal grids in 
finite difference and the requirement of a dual grid in both finite difference and finite volume.

\subsection{Galerkin Finite Element Method}

The Galerkin finite element method [36] is a specific example of the larger class of variational methods. The details of this Galerkin finite element method are best expressed through an example. Given a PDE such as Poisson's equation (3.1) an ansatz for the solution can be constructed (3.2).

$$
\begin{gathered}
\nabla \cdot D \nabla u=-f \text { on } \Omega u=0 \text { on } \Gamma \\
u_{G}=\sum_{i}^{n} u_{i} \phi_{i}(\vec{x})
\end{gathered}
$$

The ansatz is constructed by choosing $u$ to be in a space of functions $\mathcal{V}$. The space $\mathcal{V}$ is called a Sobelev space $W_{q}^{k}(\Omega)$. The Sobelev space $W_{q}^{k}(\Omega)$ is a complete, normed, inner product space of order $k$ which includes the derivatives of its members up to order $q[37]$. In this dissertation only Sobolev spaces with $q=2$ are used, these spaces are referred to as Hilbert spaces. The Hilbert spaces for the continuous $p$-forms were listed in the first chapter and will be discussed in more depth in the following section.

The basis functions $\phi_{i}(\vec{x})$ can span the entire domain $\Omega$ or can have compact support, meaning they are only defined on small subdomain. This latter case leads to the finite element method and will be used for the rest of the example.

The finite element method requires the solution domain to be decomposed into a conforming tessellation of the domain into $N_{v o l s}$ polyhedral. Over each of the subdomains the basis functions $\phi\left(\vec{x}_{i}\right)$ will be defined. Within each subdomain the 
solution $u_{f e}$ will be defined by the $n_{e}$ degrees of freedom $u_{i}$ corresponding to the $n_{e}$ nonzero basis functions defined within the subdomain. The sum of the product of all of the $n$ degrees of freedom $u_{i}$ and the $n$ basis functions $\phi(\vec{x})$ forms the Galerkin finite element solution.

The ansatz is entered into the PDE Poisson's equation to form the residual (3.3). The residual equation would be zero if $u_{G}$ is the exact solution.

$$
R\left[u_{G}\right]=-\nabla \cdot D \nabla u_{G}-f
$$

After the residual equation is constructed, moments of this equation over the entire domain are taken with the basis functions and are required to vanish (3.4).

$$
\int_{\Omega} \phi\left(\vec{x}_{i}\right) R\left[u_{G}\right]=0 \forall \phi(\vec{x})_{i}
$$

The Galerkin variational form (3.5) results from entering the residual into (3.5).

$$
-(\nabla \cdot D \nabla u, v)=(f, v) \forall v \in \mathcal{T}=\operatorname{Span}\left\{\phi(\vec{x})_{i}\right\}
$$

Here the notation $($,$) is the integration over the entire domain (\alpha, \beta)=\int_{\Omega} \alpha \beta d \Omega$ for scalars and $(\vec{\alpha}, \vec{\beta})=\int_{\Omega} \vec{\alpha} \cdot \vec{\beta} d \Omega$ for vectors. The generalization of the operators in (3.5) are commonly referred to as a bilinear forms. The bilinear forms can be defined for vectors and differential operators and will be discussed in more detail below.

In general the space $u$ and the space $v$ need not be the same. In this dissertation the Solution Space $\mathcal{V}$ and the test space $\mathcal{T}$ are the same space. The test space $\mathcal{T}$ only differs from $\mathcal{V}$ by the inclusion of the boundary condition.

This Galerkin variational form leads to a discrete system of equations for $u$ given by $(3.6)$.

$$
\mathbf{A} \vec{u}=\vec{b}
$$


Where $\vec{u}$ are the vector of coefficients used in $(3.2), \vec{b}$ is vector created by $(f, v)$ and A is the matrix resulting from $(\nabla \cdot D \nabla u, v)$.

The benefits of using the Galerkin finite element method over a variational method such as the Rayleigh-Ritz variational method include the ability to define the variational method for non-self-adjoint PDEs and reducing to the optimum Rayleigh-Ritz variational form for self-adjoint PDEs.

\section{$3.2 \quad$ Vector Spaces}

The Galerkin variational method requires each variable be defined in Hilbert space. This section defines the solution and test spaces for each of the $p$-forms .

The 3-forms in this dissertation are element centered quantities that have no continuity. The proper space defining these functions is (3.7) with its associated norm given in (3.8). The test and solution space for this form are the same. The total number of basis functions in which to expand the discrete 3 -form is the total number of elements in the mesh $N_{\text {vols }}$.

$$
\begin{gathered}
L^{2}(\Omega)=\left\{u ; \int_{\Omega} u^{2} d \Omega<\infty\right\} \\
\|u\|_{L^{2}}=\left(\|u\|^{2}\right)^{1 / 2}
\end{gathered}
$$

The 0-forms are totally continuous scalar functions with the gradient as the differential operator. The proper space to define these functions is $\mathcal{H}($ grad $)$ defined in (3.9). The test space for the 0 -forms has the homogeneous Dirichlet boundary condition $u=0$ on $\Gamma$ as a constraint (3.10). The norm for the space is given in 
(3.11).

$$
\begin{gathered}
\mathcal{H}(\operatorname{grad}, \Omega)=\left\{u: u \in L^{2}(\Omega) ; \nabla u \in\left(L^{2}(\Omega)\right)^{3}\right\} \\
\mathcal{H}_{0}(\operatorname{grad}, \bar{\Omega})=\left\{u: u \in L^{2}(\Omega) ; u=0 \text { on } \Gamma\right\} \\
\|u\|_{\mathcal{H}(\operatorname{grad}, \Omega)}=\left(\|u\|^{2}+\|\nabla u\|^{2}\right)^{1 / 2}
\end{gathered}
$$

In electrodynamics the electric field has tangential continuity across a material boundary. In this case it would be improper to force the electric field to have total continuity across the material boundary by treating each component as a totally continuous scalar function. The inclusion relation (2.77) shows that the space of the curl of the 1 -forms is included in the 2 -forms space. Is necessary to include the curl of the discrete 1-forms in the space used to represent them in order to maintain this inclusion relation on the discrete level. This space is given by $\mathcal{H}(\mathrm{curl})$ (3.12). The test space for the 1-forms include the homogeneous vector Dirichlet boundary condition $\vec{u} \times \hat{n}=0$ on $\Gamma$ (3.13). The norm for $\mathcal{H}($ curl) is given in (3.14).

$$
\begin{gathered}
\mathcal{H}(\text { curl }, \Omega)=\left\{\vec{u}: \vec{u} \in\left(L^{2}(\Omega)\right)^{3} ; \nabla \times \vec{u} \in\left(L^{2}(\Omega)\right)^{3}\right\} \\
\mathcal{H}_{0}(\operatorname{curl}, \bar{\Omega})=\left\{\vec{u}: \vec{u} \in\left(L^{2}(\Omega)\right)^{3} ; \hat{n} \times \vec{u}=0 \text { on } \Gamma\right\} \\
\|\vec{u}\|_{\mathcal{H}(\text { curl }, \Omega)}=\left(\|\vec{u}\|^{2}+\|\nabla \times \vec{u}\|^{2}\right)^{1 / 2}
\end{gathered}
$$

In electrodynamics the magnetic flux density has normal continuity across a material boundary. Just as in this case with the electric field it would be improper to force the magnetic flux density to have total continuity across this boundary. The continuity of the 2 -forms requires that these forms be defined in the space $\mathcal{H}($ div) (3.15). The test space for the 2-forms include the homogeneous vector Dirichlet boundary condition $\vec{u} \cdot \hat{n}=0$ on $\Gamma$ (3.16). The norm for $\mathcal{H}($ div $)$ is given in (3.17). 


$$
\begin{gathered}
\mathcal{H}(\operatorname{div}, \Omega)=\left\{\vec{u}: \vec{u} \in\left(L^{2}(\Omega)\right)^{3} ; \nabla \cdot \vec{u} \in L^{2}(\Omega)\right\} \\
\mathcal{H}_{0}(\operatorname{div}, \bar{\Omega})=\left\{\vec{u}: \vec{u} \in\left(L^{2}(\Omega)\right)^{3} ; \vec{u} \cdot \hat{n}=0 \text { on } \Gamma\right\} \\
\|\vec{u}\|_{\mathcal{H}(\operatorname{div}, \Omega)}=\left(\|\vec{u}\|^{2}+\|\nabla \cdot \vec{u}\|^{2}\right)^{1 / 2}
\end{gathered}
$$

Requiring the discrete forms be defined in these spaces satisfies the first requirement of a discrete differential forms framework.

\subsection{Finite Elements}

The Galerkin finite element method is the restriction of the Galerkin variational method to the expansion of variables in terms of functions with compact support. These functions with compact support span a polynomial space with dimension $\mathcal{D}$ referred to as $\mathcal{P}$. The degrees of freedom $\mathcal{A}$ determine a basis $\left\{\Psi_{1}, \Psi_{2}, \ldots, \Psi_{N}\right\}$ in which the variable can be expanded. The basis functions are non-zero only within a specified polyhedral domain $\mathcal{K}$. The combination of $(\mathcal{K}, \mathcal{P}, \mathcal{A})$ forms a finite element.

The four $p$-forms require four polynomial spaces, four basis functions and four associated degrees of freedom representations to form discrete differential forms. The four basis functions correspond to a nodal basis functions for the 0-form and cellcentered basis functions for the 3-form as described in the mixed finite element method of Raviart-Thomas. Two new basis functions for three dimensions developed by Nédélecform the basis for the discrete 1-form and 2-form. The degrees of freedom represent the discrete $p$-forms coefficients as demonstrated for the continuous case in Chapter 2. The polynomial spaces $\mathcal{P}$, basis functions and degrees of 
freedom for tetrahedral,prismatic and hexahedral domains are presented below. The essential properties of the $p$-form basis functions and degrees of freedom are discussed in [10], [38], [15].

In this dissertation the basis functions are defined on a reference element $\tilde{\mathcal{E}}=$ $(\tilde{\mathcal{K}}, \tilde{\mathcal{P}}, \tilde{\mathcal{A}})$ and mapped to an arbitrary finite element $\mathcal{E}_{i}=\left(\mathcal{K}_{i}, \mathcal{P}_{i}, \mathcal{A}_{i}\right)$ through an isoparametric mapping $\Phi(\tilde{\mathcal{K}})=\mathcal{K}$. The reference element polyhedral domains $\tilde{\mathcal{K}}$ correspond to the unit cube, tetrahedron or prism.

The Galerkin finite element method requires a conforming mesh $T$. The two requirements for a conforming mesh include a non-overlapping collection of threedimensional polyhedra $\mathcal{K}_{i}$ that intersect only on the faces $\Gamma_{i j}$ of the polyhera and fill the entire domain (3.19).

$$
\begin{aligned}
\mathcal{K}_{i} \cap \mathcal{K}_{j} & =\Gamma_{i j} \\
\cup \mathcal{K}_{i} & =\Omega
\end{aligned}
$$

Due to the compact support of the finite element basis functions two interpolants can be defined. The local interpolant for an arbitrary variable $f(3.20)$ is the sum of the product of the $N$ local degrees of freedom and local basis functions.

$$
I_{\mathcal{K}} f=\sum_{i=1}^{N} \alpha_{i}(f) \Psi_{i}
$$

Global basis functions are formed by the local basis functions that are non-zero within an element and zero everywhere else. Using these global basis functions a global interpolant for an arbitrary function $f$ can be defined (3.21) which is a sum of the local interpolants over the entire domain.

$$
\left.I_{T} f\right|_{\mathcal{K}_{i}}=I_{\mathcal{K}_{i}} f \quad \forall \mathcal{K}_{i} \in T
$$


The order $q$ of continuity of the global interpolant is defined by $I_{T} f \in C^{q} \forall f \in$ $C^{m} m \geq q$.

The error involved with the global interpolation can be bound by the parameter $h_{i}$ which is the maximum diameter of the polyhedral domain $\mathcal{K}_{i}$. A sequence of meshes is refined as $h \rightarrow 0$. If all the angles in the mesh are bound between a nonzero minimum and a maximum less than $\pi$ then as $h \rightarrow 0$ the meshes are said to be refined uniformly. The approximation error for a polynomial space $\mathcal{P}$ complete to order $k-1$ and a global interpolant continuous to order $q$ [36] [39] is given in (3.22).

$$
\left|f-I_{T} f\right|_{W_{p}^{s}} \leq C_{s} h^{k-s}|f|_{W_{p}^{k}} \quad ; s \leq q
$$

\subsubsection{Degrees of freedom}

The degrees of freedom $\mathcal{A}$ are a subset of the dual space of $\mathcal{P}_{i}$, which is a set of linear functionals from $\mathcal{P}_{i}$ onto $\Re$ [40]. Several criteria must be enforced to have valid degrees of freedom, they must be unisolvent, invariant and local. Unisolvence refers to the criteria that the degrees of freedom, $\left\{\alpha_{i}\right\}$, be dual to $\mathcal{P}_{i}(3.23)$.

$$
\alpha_{i}\left(\psi_{j}\right)=\delta_{i j}
$$

Invariance refers to the property in which under a transformation of variables the degrees of freedom remain unisolvent. This becomes important when the basis functions are transformed from the reference element to the physical domain via the pullback operator. The locality of the degrees of freedom refers to the criteria that the trace of the basis function on a sub-simplex is determined by the degrees of freedom associated only with the sub-simplex. 
The general definition of the degrees of freedom $\left\{\alpha_{i}\right\}$ is given in (3.24)

$$
\left\{\alpha_{i}\right\}=\left\{\psi \rightarrow \int_{s} \psi \wedge \eta_{i, s}\right\}
$$

In this equation the value for $s$ denotes the sub-simplex, i.e. node, edge, face or volume, and $\left\{\eta_{i, s}\right\}$ are a set of weighting polynomials. For the lowest order basis functions presented in this dissertation the degrees of freedom for the $p$-forms are presented in Table 3.1.

Table 3.1: Degrees of Freedom

\begin{tabular}{|c|c|}
\hline Form & Degree of Freedom \\
\hline \hline 0 & $\int_{\text {node }} \psi \delta($ node $)$ dvolume \\
\hline 1 & $\int_{\text {edge }} \vec{\psi} \times \hat{n}$ dedge \\
\hline 2 & $\int_{\text {face }} \vec{\psi} \cdot \hat{n} d$ face \\
\hline 3 & $\int_{\text {volume }} \psi d$ dvolume \\
\hline
\end{tabular}

In this table the 0 -form degree of freedom is the value of the function at the node given by the integration involving the delta function at the given node.

The degrees of freedom shown in Table 3.1 satisfy the requirement that they be related to the traces of the forms. Each $p$-form is the $p$-fold integral over the domain.

\subsubsection{Linear tetrahedral basis functions}

The linear tetrahedral element illustrated in Figure 3.1 has the four polynomial spaces $\mathcal{P}_{i}(3.25),(3.26),(3.27),(3.28)$ associated with the four discrete differential forms. The dimensions of each of the spaces is $\{4,6,4,1\}$ respectively, corresponding to the number of nodes, edges, faces and volumes contained in the element.

$$
\mathcal{P}_{0}=\left\{u: u \in \mathbf{P}_{1} ; u=a_{0}+a_{1} \psi+a_{2} \xi+a_{3} \eta ; a_{i} \in \Re\right\}
$$



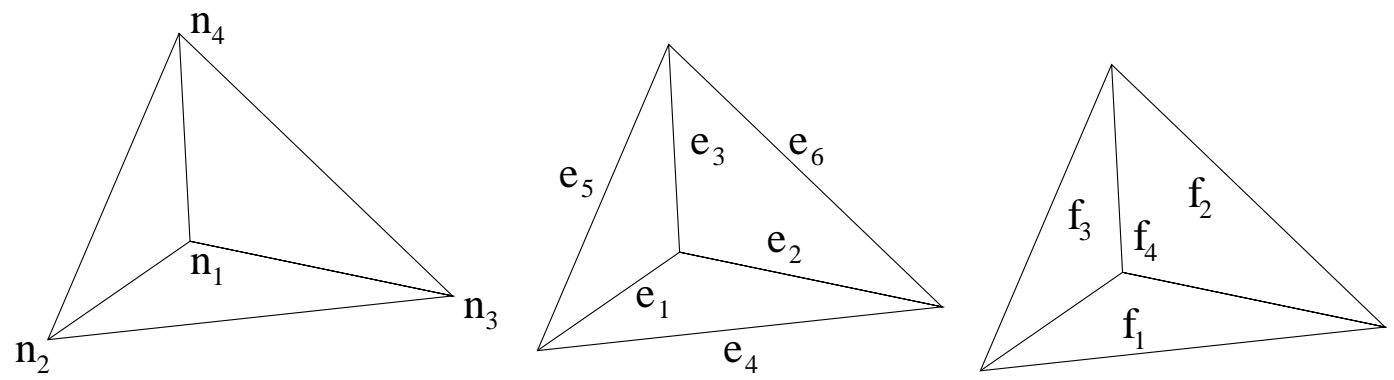

Figure 3.1: Tetrahedron Basis Function Numbering

$$
\begin{gathered}
\mathcal{P}_{1}=\left\{\vec{u}: \vec{u}=\left(u_{1}, u_{2}, u_{3}\right) \vec{u} \in\left(\mathbf{P}_{1}\right)^{3} ; \frac{\partial u_{i}}{\partial x_{j}} \delta_{i j}=0 ; \frac{\partial u_{i}}{\partial x_{j}}+\frac{\partial u_{j}}{\partial x_{i}}=0, i \neq j\right\} \\
\mathcal{P}_{2}=\left\{\vec{u}: \vec{u} \in\left(\left(\mathbf{P}_{0}\right)^{3}+\mathbf{P}_{0} \cdot \vec{r}\right) ; \vec{u}=\left\{a_{0}+a_{3} \psi, a_{1}+a_{3} \xi, a_{2}+a_{3} \eta\right\} ; a_{i} \in \Re\right\} \\
\mathcal{P}_{3}=\left\{u: u \in \mathbf{P}_{0} ; u=a_{0} ; a_{0} \in \Re\right\}
\end{gathered}
$$

The four sets of tetrahedron basis functions are listed in Table 3.2 and are constructed by forcing the condition (3.23) for each of the degrees of freedom listed in Table 3.1. These basis functions are defined on a tetrahedral reference element with coordinates $\{\psi, \xi, \eta\}$.

Table 3.2: Tetrahedral Basis Functions

\begin{tabular}{|c||c|c|c|c|}
\hline & $\mathcal{N}$ Nodal & $\mathcal{W}$ Edge & $\mathcal{F}$ Face & $\mathcal{S}$ Volume \\
\hline \hline 1 & $(1-\psi-\xi-\eta)$ & $\{(1-\xi-\eta), \psi, \psi\}$ & $\{-2 \psi,-2 \xi, 2(1-\eta)\}$ & constant \\
\hline 2 & $\psi$ & $\{\xi,(1-\psi-\eta), \xi\}$ & $\{2 \psi, 2(\xi-1), 2 \eta\}$ & - \\
\hline 3 & $\xi$ & $\{\eta, \eta,(1-\psi-\xi)\}$ & $\{2(1-\psi),-2 \xi,-2 \eta\}$ & - \\
\hline 4 & $\eta$ & $\{-\xi, \psi, 0\}$ & $\{2 \psi, 2 \xi, 2 \eta\}$ & - \\
\hline 5 & - & $\{-\eta, 0, \psi\}$ & - & - \\
\hline 6 & - & $\{0,-\eta, \xi\}$ & - & - \\
\hline
\end{tabular}




\section{0-form basis}

The four local 0-form basis functions referred to as the nodal basis functions are shown in Figure 3.2. For a mesh with $N_{\text {vols }}$ tetrahedrons containing $N_{\text {nodes }}$ nodes
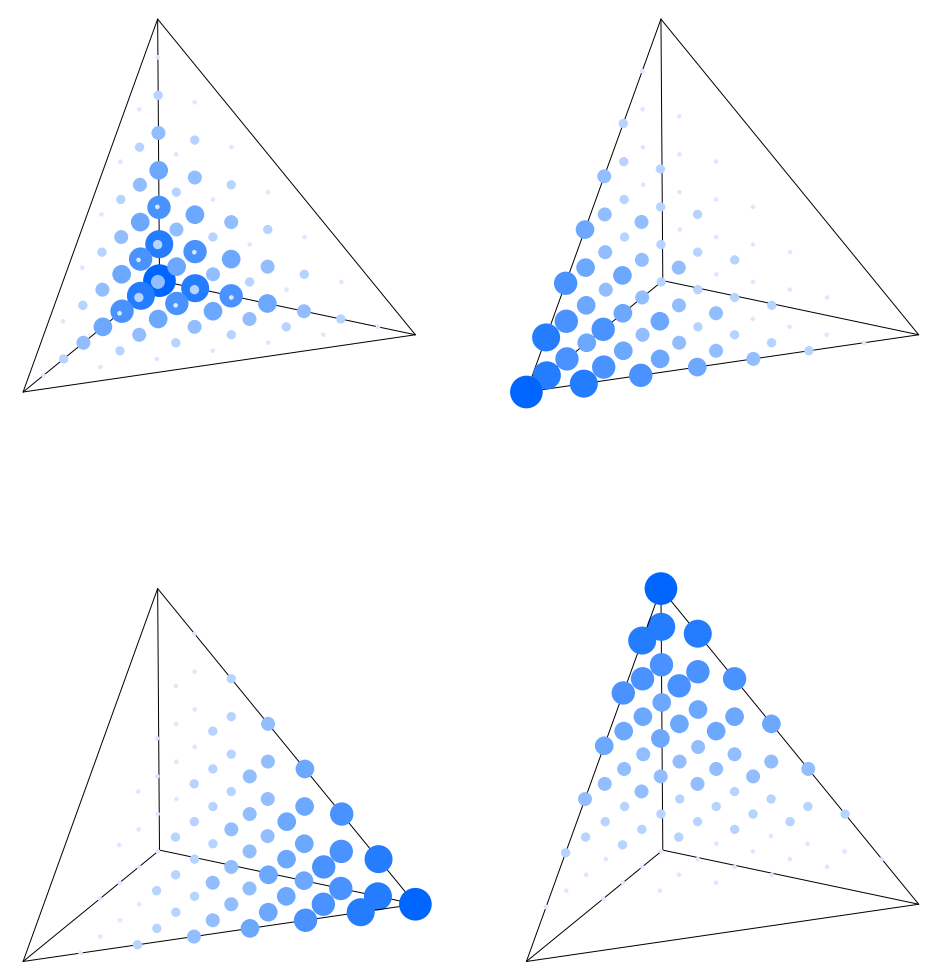

Figure 3.2: Tetrahedron Node Basis Functions

there is a linear nodal finite element $\left(\mathcal{K}_{i}, \mathcal{P}_{i}, \mathcal{A}_{i}\right)$ associated with each element. An arbitrary function $f$ can be expanded in $N_{\text {nodes }}$ global basis functions consisting of the local basis functions listed in the first column of Table 3.2 inside the element and zero outside. The collection of all of these global basis functions is referred to as $\mathcal{N}^{h}$ with dimension $N_{\text {nodes }}$. The value of $f$ is determined by the four values at the four nodes. Because the nodes are shared between adjoining elements the value of $f$ must 
be consistent giving a continuous function. The rate of convergence for this finite element is $(3.29)$.

$$
\left|f-I_{T} f\right|_{2} \leq C h^{2}|f|_{\mathcal{H}^{2}}
$$

\section{1-form basis}

The six local 1-form basis functions referred to as the edge basis functions are shown in Figure 3.3. For a mesh with $N_{\text {vols }}$ tetrahedrons containing $N_{\text {edges }}$ edges there is a linear edge finite element $\left(\mathcal{K}_{i}, \mathcal{P}_{i}, \mathcal{A}_{i}\right)$ associated with each element. An arbitrary function $f$ can be expanded in $N_{\text {edges }}$ global basis functions consisting of the local basis functions listed in the second column of Table 3.2 inside the element and zero outside. The collection of all of these global basis functions is referred to as $\mathcal{W}^{h}$ with dimension $N_{\text {edges }}$. The value of $f$ is determined by the six values at the six edges. The edge basis functions maintain tangential continuity by construction. The rate of convergence for this finite element is (3.30).

$$
\left|f-I_{T} f\right|_{\mathcal{H}(\text { curl })} \leq C h^{1}|f|_{\mathcal{H}^{1}}
$$

\section{2-form basis}

The four local 2-form basis functions referred to as the face basis functions are shown in Figure 3.4. For a mesh with $N_{\text {vols }}$ tetrahedrons containing $N_{\text {faces }}$ faces there is a linear face finite element $\left(\mathcal{K}_{i}, \mathcal{P}_{i}, \mathcal{A}_{i}\right)$ associated with each element. An arbitrary function $f$ can be expanded in $N_{\text {faces }}$ global basis functions consisting of the local basis functions listed in the third column of Table 3.2 inside the element and zero

outside. The collection of all of these global basis functions is referred to as $\mathcal{F}^{h}$ with 

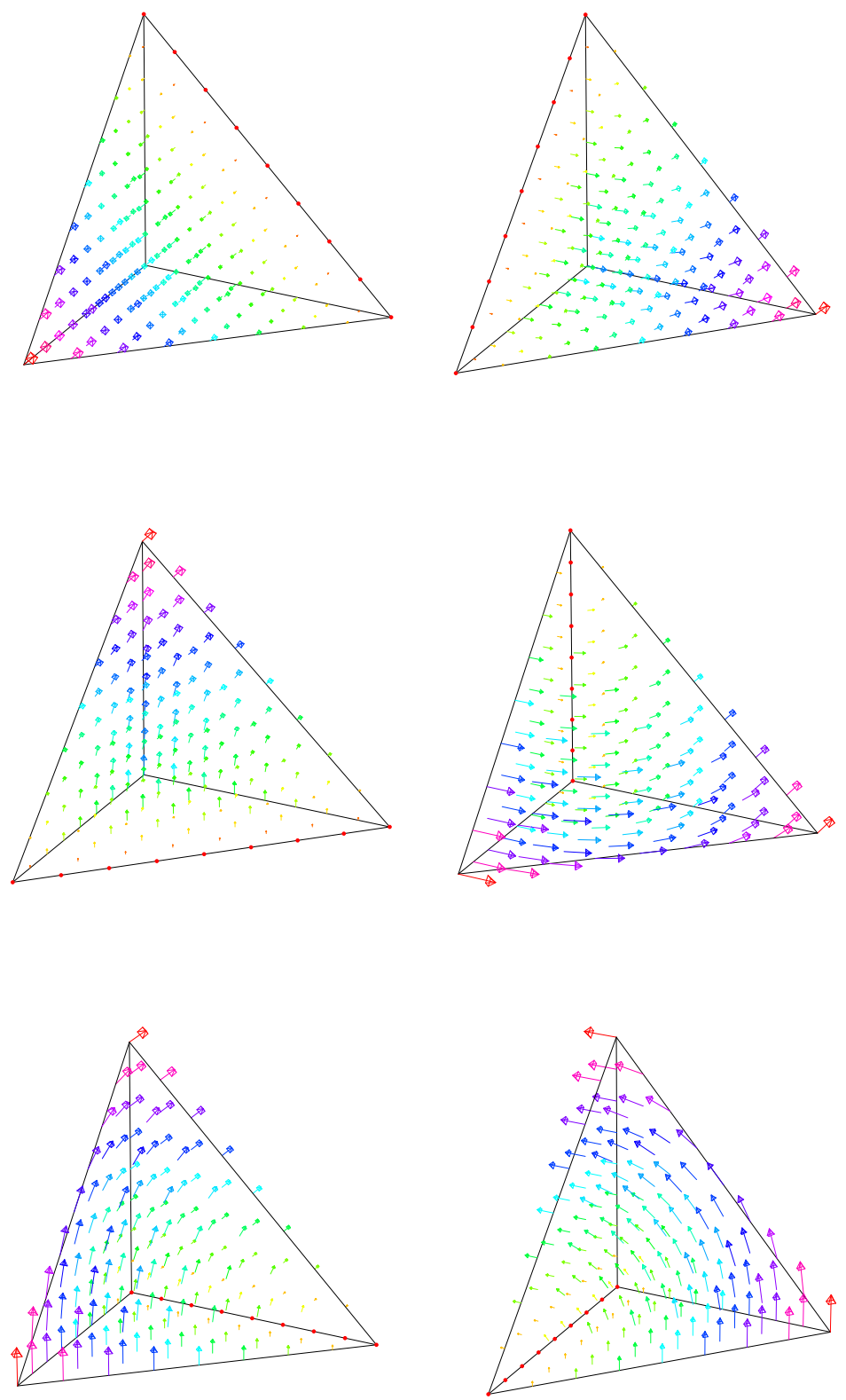

Figure 3.3: Tetrahedron Edge Basis Functions 

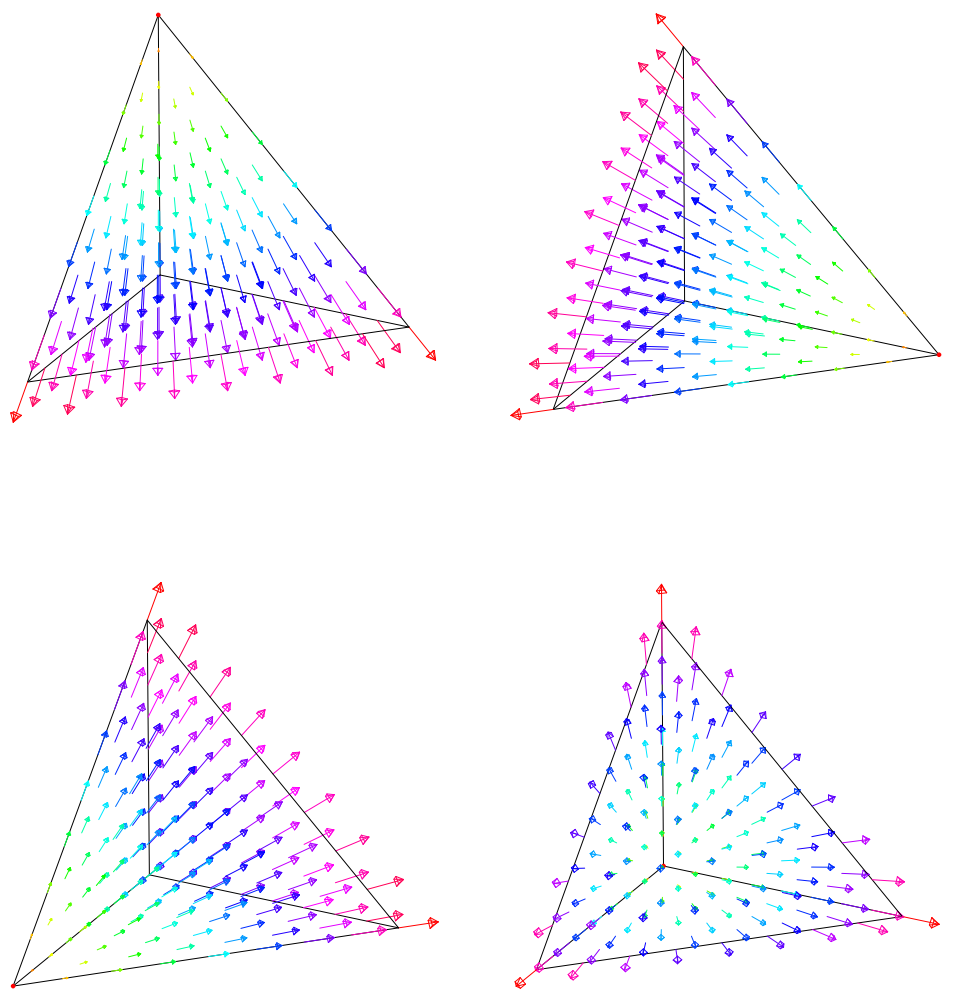

Figure 3.4: Tetrahedron Face Basis Functions 
dimension $N_{\text {faces }}$. The value of $f$ is determined by the four values at the four faces. The face basis functions maintain normal continuity by construction. The rate of convergence for this finite element is (3.31).

$$
\left|f-I_{T} f\right|_{\mathcal{H}(\text { div })} \leq C h^{1}|f|_{\mathcal{H}^{1}}
$$

\section{3-form basis}

The single constant local 3-form basis functions referred to as the volume basis function is shown in Figure 3.5. For a mesh with $N_{\text {vols }}$ tetrahedrons there is a linear

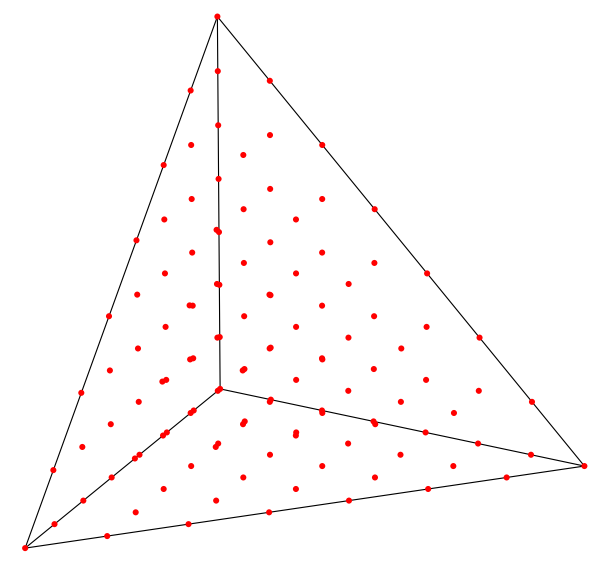

Figure 3.5: Tetrahedron Volume Basis Functions

face finite element $\left(\mathcal{K}_{i}, \mathcal{P}_{i}, \mathcal{A}_{i}\right)$ associated with each element. An arbitrary function $f$ can be expanded in $N_{\text {vols }}$ global basis functions consisting of the local basis functions listed in the fourth column of Table 3.2 inside the element and zero outside. The collection of all of these global basis functions is referred to as $\mathcal{S}^{h}$ with dimension $N_{\text {vols }}$. The value of $f$ is determined by the four values at the four faces. The volume basis functions have no continuity. The rate of convergence for this finite element is 
$(3.32)$.

$$
\left|f-I_{T} f\right|_{2} \leq C h^{1}|f|_{\mathcal{H}^{1}}
$$

\subsubsection{Linear prismatic basis functions}

The linear prismatic element illustrated in Figure 3.6
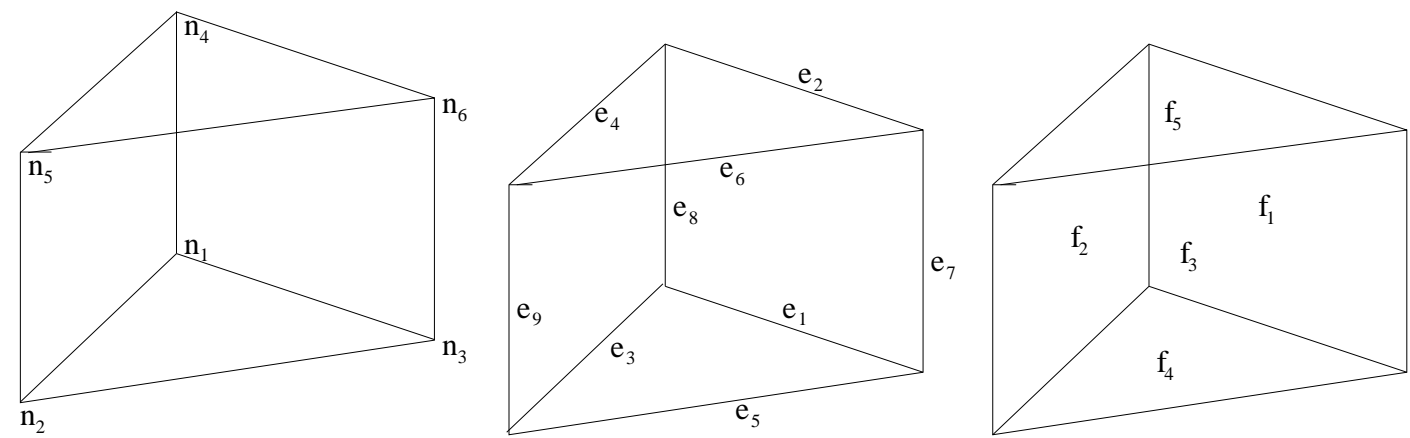

Figure 3.6: Prism Basis Function Numbering

has the four polynomial spaces $\mathcal{P}_{i}(3.33),(3.34),(3.35),(3.36)$ associated with the four discrete differential forms. The dimensions of each of the spaces is $\{5,9,5,1\}$ respectively, corresponding to the number of nodes, edges, faces and volumes contained in the element.

$$
\begin{gathered}
\mathcal{P}_{0}=\left\{u: u \in \mathbf{P}_{1} ; u=a_{0}+a_{1} \psi+a_{2} \xi+a_{3} \eta+a_{4} \psi \eta+a_{5} \xi \eta ; a_{i} \in \Re\right\} \\
\mathcal{P}_{1}=\left\{\vec{u}: \vec{u}=\left(u_{1}, u_{2}, u_{3}\right) ; u_{1} \in\left(a_{0}+a_{1} \xi\right)\left(a_{2}+a_{3} \eta\right)\right. \\
\left.u_{2} \in\left(a_{4}+a_{5} \psi\right)\left(a_{2}+a_{3} \eta\right), u_{3} \in\left(a_{6}+a_{7} \psi+a_{8} \xi\right)\right\} \\
\mathcal{P}_{2}=\left\{\vec{u}: \vec{u}=\left(u_{1}, u_{2}, u_{3}\right) ; u_{1} \in\left(a_{0}+a_{2} \psi\right), u_{2} \in\left(a_{1}+a_{2} \xi\right), u_{3} \in\left(a_{3}+a_{4} \eta\right)\right\}
\end{gathered}
$$




$$
\mathcal{P}_{3}=\left\{u: u \in \mathbf{P}_{0} ; u=a_{0} ; a_{0} \in \Re\right\}
$$

The four sets of Prism basis functions are listed in Table 3.3.

Table 3.3: Prismatic Basis Functions

\begin{tabular}{|c||c|c|c|c|}
\hline & $\mathcal{N}$ Nodal & $\mathcal{W}$ Edge & $\mathcal{F}$ Face & $\mathcal{S}$ Volume \\
\hline \hline 1 & $(1-\psi-\xi-\eta)(1-\eta)$ & $\{-\xi(1-\eta),(1-\eta)(\psi-1), 0\}$ & $\{(\psi-1), \xi, 0\}$ & constant \\
\hline 2 & $\psi(1-\eta)$ & $\{\eta \xi, \eta(1-\psi), 0\}$ & $\{\psi,(\xi-1), 0\}$ & - \\
\hline 3 & $\eta(1-\eta)$ & $\{(1-\xi)(1-\eta), \psi(1-\eta), 0\}$ & $\{\psi, \xi, 0\}$ & - \\
\hline 4 & $(1-\psi-\xi) \eta$ & $\{\eta(\xi-1),-\psi \eta, 0\}$ & $\{0,0,(\eta-1)\}$ & - \\
\hline 5 & $\psi \eta$ & $\{-\xi(1-\eta), \psi(1-\eta), 0\}$ & $\{0,0, \eta\}$ & - \\
\hline 6 & $\xi \eta$ & $\{\xi \eta,-\psi \eta, 0\}$ & - & - \\
\hline 7 & - & $\{0,0,-\xi\}$ & - & - \\
\hline 8 & - & $\{0,0,(1-\psi-\xi)\}$ & - & - \\
\hline 9 & - & $\{0,0, \psi\}$ & - & - \\
\hline
\end{tabular}

\section{0-form basis}

The six local 0-form basis functions referred to as the nodal basis functions are shown in Figure 3.7. For a mesh with $N_{\text {vols }}$ prisms containing $N_{\text {nodes }}$ nodes there is a linear nodal finite element $\left(\mathcal{K}_{i}, \mathcal{P}_{i}, \mathcal{A}_{i}\right)$ associated with each element. An arbitrary function $f$ can be expanded in $N_{\text {nodes }}$ global basis functions consisting of the local basis functions listed in the first column of Table 3.3 inside the element and zero outside. The collection of all of these global basis functions is referred to as $\mathcal{N}^{h}$ with dimension $N_{\text {nodes }}$. The value of $f$ is determined by the four values at the four nodes. Because the nodes are shared between adjoining elements the value of $f$ must be consistent giving a continuous function. The rate of convergence for this finite element is (3.37).

$$
\left|f-I_{T} f\right|_{2} \leq C h^{2}|f|_{\mathcal{H}^{2}}
$$



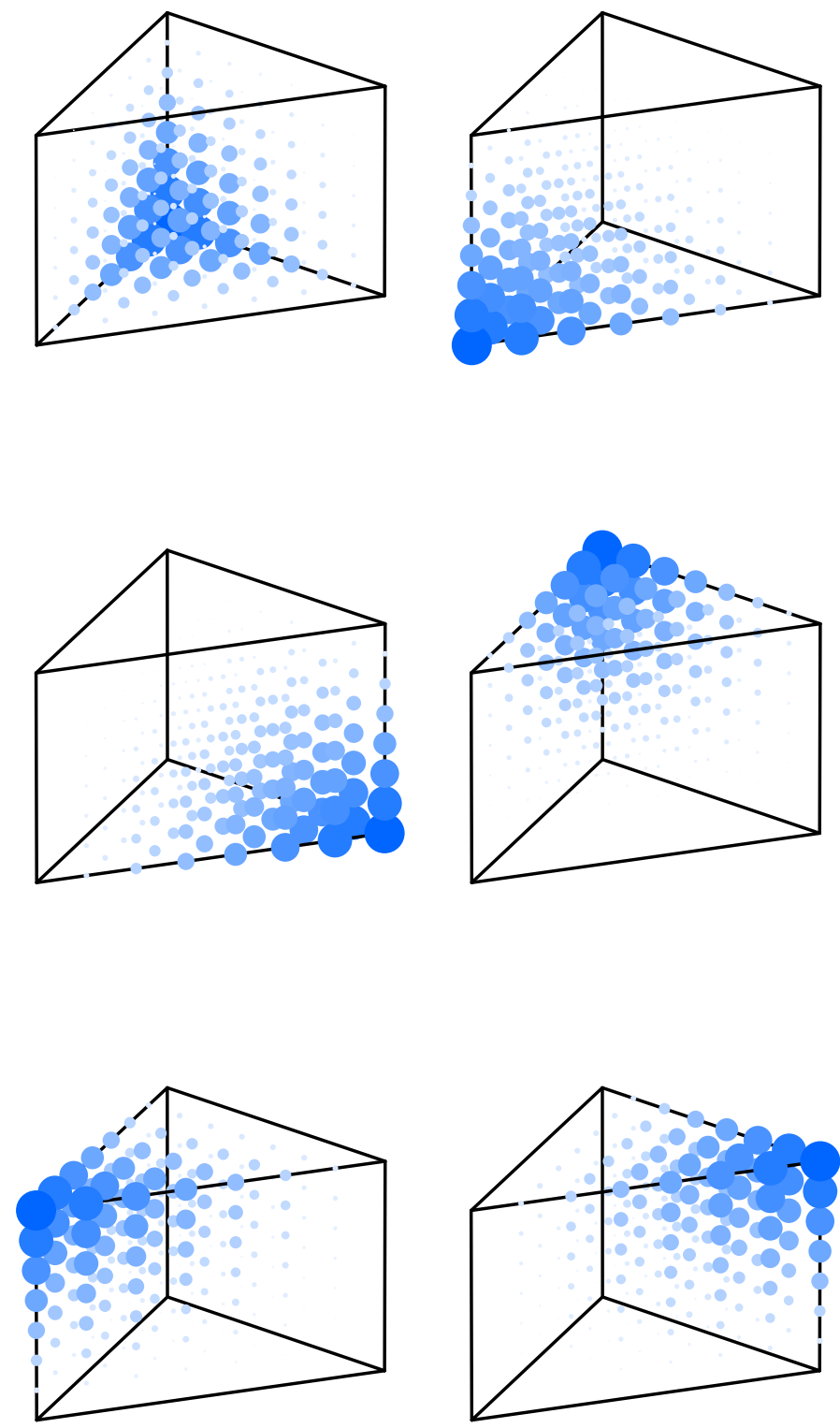

Figure 3.7: Prism Node Basis Functions 


\section{1-form basis}

The nine local 1-form basis functions referred to as the edge basis functions are

shown in Figure 3.8. For a mesh with $N_{\text {vols }}$ prisms containing $N_{\text {edges }}$ edges there is
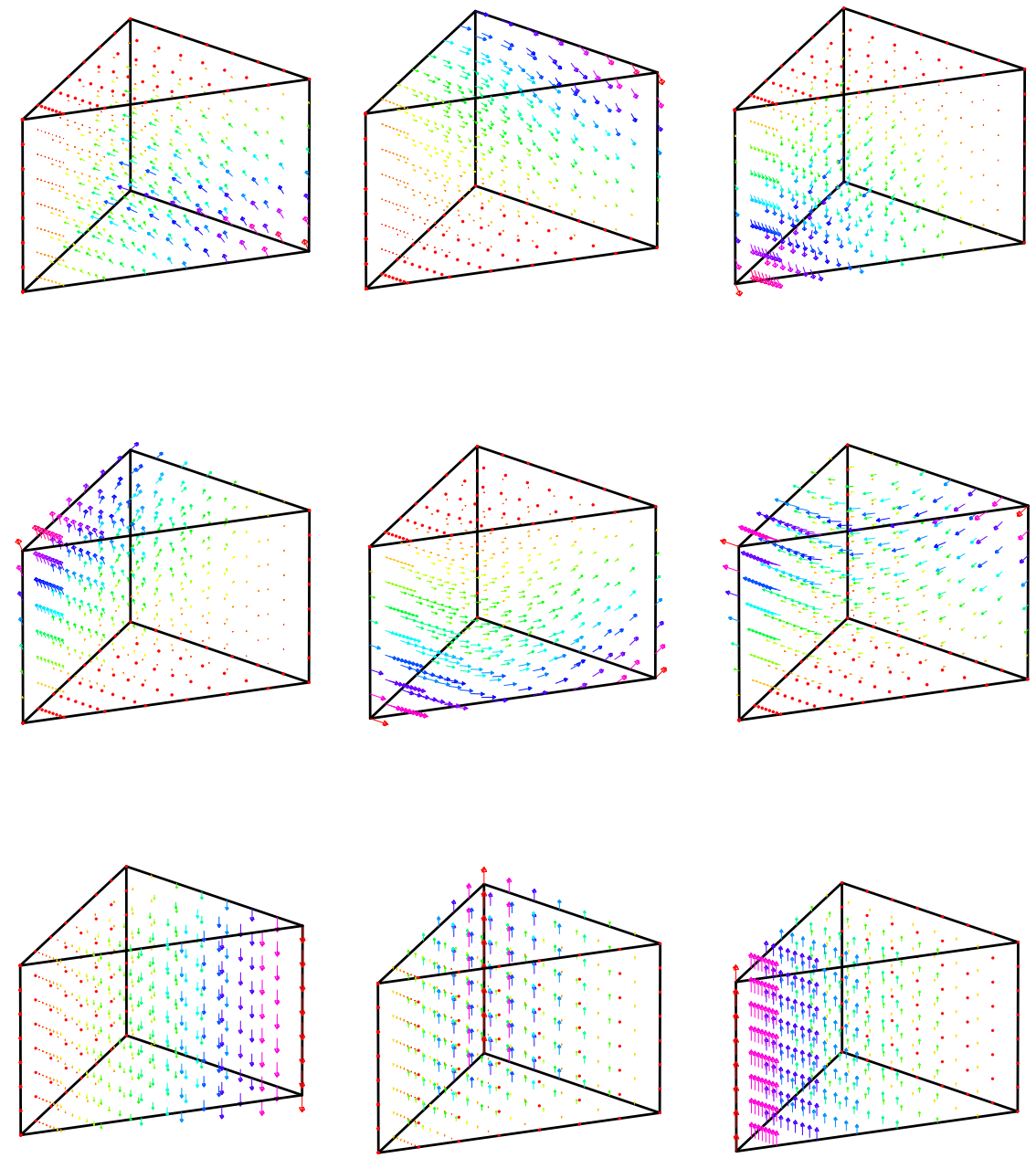

Figure 3.8: Prism Edge Basis Functions

a linear edge finite element $\left(\mathcal{K}_{i}, \mathcal{P}_{i}, \mathcal{A}_{i}\right)$ associated with each element. An arbitrary function $f$ can be expanded in $N_{\text {edges }}$ global basis functions consisting of the local basis functions listed in the second column of Table 3.3 inside the element and zero 
outside. The collection of all of these global basis functions is referred to as $\mathcal{W}^{h}$ with dimension $N_{\text {edges }}$. The value of $f$ is determined by the six values at the six edges. The edge basis functions maintain tangential continuity be construction. The rate of convergence for this finite element is (3.38).

$$
\left|f-I_{T} f\right|_{\mathcal{H}(\text { curl })} \leq C h^{1}|f|_{\mathcal{H}^{1}}
$$

\section{2-form basis}

The five local 2-form basis functions referred to as the face basis functions are shown in Figure 3.9. For a mesh with $N_{\text {vols }}$ prisms containing $N_{\text {faces }}$ faces there is a linear face finite element $\left(\mathcal{K}_{i}, \mathcal{P}_{i}, \mathcal{A}_{i}\right)$ associated with each element. An arbitrary function $f$ can be expanded in $N_{\text {faces }}$ global basis functions consisting of the local basis functions listed in the third column of Table 3.3 inside the element and zero outside. The collection of all of these global basis functions is referred to as $\mathcal{F}^{h}$ with dimension $N_{\text {faces }}$. The value of $f$ is determined by the four values at the four faces. The face basis functions maintain normal continuity by construction. The rate of convergence for this finite element is (3.39).

$$
\left|f-I_{T} f\right|_{\mathcal{H}(\text { div })} \leq C h^{1}|f|_{\mathcal{H}^{1}}
$$

\section{3-form basis}

The single constant local 3-form basis functions referred to as the volume basis function is shown in Figure 3.10. For a mesh with $N_{\text {vols }}$ prisms there is a linear face finite element $\left(\mathcal{K}_{i}, \mathcal{P}_{i}, \mathcal{A}_{i}\right)$ associated with each element. An arbitrary function $f$ can be expanded in $N_{\text {vols }}$ global basis functions consisting of the local basis functions 

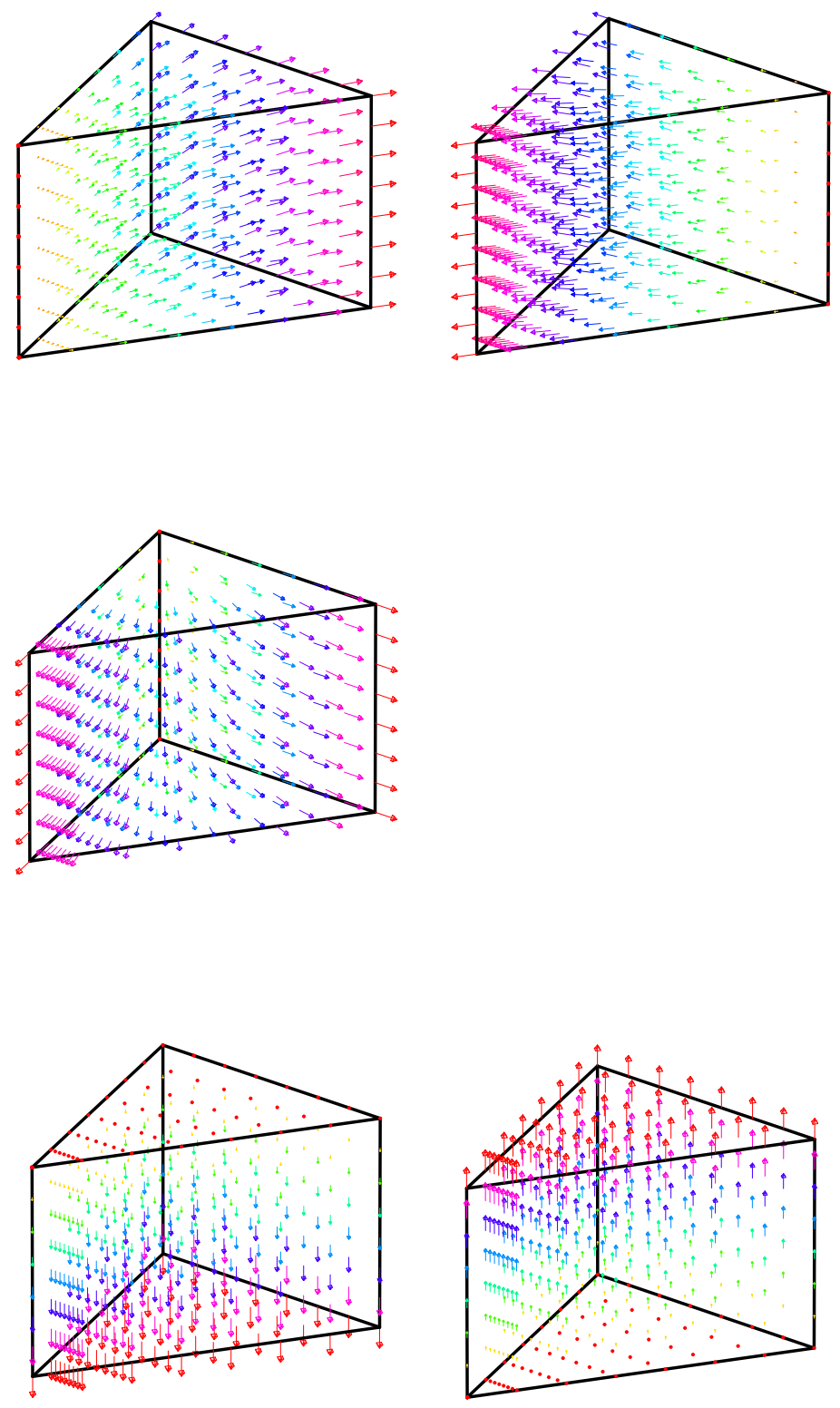

Figure 3.9: Prism Face Basis Functions 


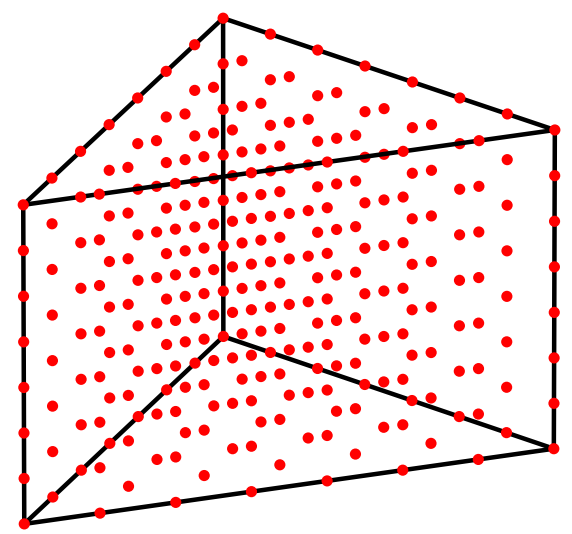

Figure 3.10: Prism Volume Basis Functions

listed in the fourth column of Table 3.3 inside the element and zero outside. The collection of all of these global basis functions is referred to as $\mathcal{S}^{h}$ with dimension $N_{\text {vols }}$. The value of $f$ is determined by the four values at the four faces. The volume basis functions have no continuity. The rate of convergence for this finite element is $(3.40)$.

$$
\left|f-I_{T} f\right|_{2} \leq C h^{1}|f|_{\mathcal{H}^{1}}
$$

\subsubsection{Linear hexahedral basis functions}

$$
\begin{gathered}
\mathbf{Q}_{1,1,1}=a_{0}+a_{1} \psi+a_{2} \xi+a_{3} \eta+a_{4} \psi \xi+a_{5} \psi \eta+a_{6} \xi \eta+a_{7} \psi \xi \eta \\
\mathcal{P}_{0}=\left\{u: u \in \mathbf{Q}_{1,1,1}\right\} \\
\mathcal{P}_{1}=\left\{\vec{u}: \vec{u}=\left(u_{1}, u_{2}, u_{3}\right) ; u_{1} \in \mathbf{Q}_{0,1,1} ; u_{2} \in \mathbf{Q}_{1,0,1} ; u_{3} \in \mathbf{Q}_{1,1,0}\right\} \\
\mathcal{P}_{2}=\left\{\vec{u}: \vec{u}=\left(u_{1}, u_{2}, u_{3}\right) ; u_{1} \in \mathbf{Q}_{1,0,0} ; u_{2} \in \mathbf{Q}_{0,1,0} ; u_{3} \in \mathbf{Q}_{0,0,1}\right\}
\end{gathered}
$$




$$
\mathcal{P}_{3}=\left\{u: u \in \mathbf{P}_{0} ; u=a_{0} ; a_{0} \in \Re\right\}
$$

The four sets of Hexahedral basis functions are listed in Table 3.4. The numbering for nodes, edges and faces is given in Figure 3.11.
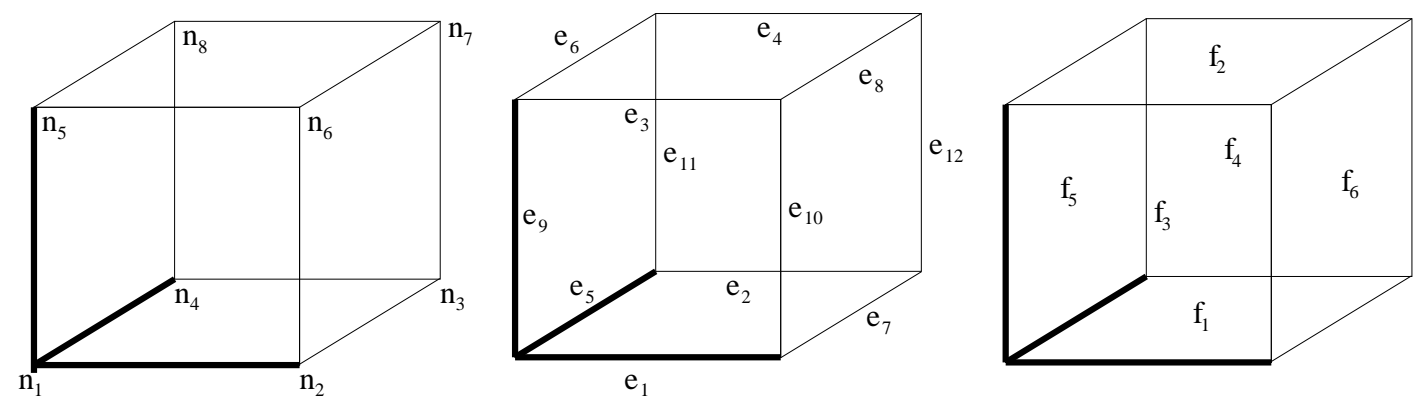

Figure 3.11: Hexahedral Basis Function Numbering

Table 3.4: Hexahedral Basis Functions

\begin{tabular}{|c||c|c|c|c|}
\hline & $\mathcal{N}$ Nodal & $\mathcal{W}$ Edge & $\mathcal{F}$ Face & $\mathcal{S}$ Volume \\
\hline \hline 1 & $(1-\psi)(1-\xi)(1-\eta)$ & $\{(1-\xi)(1-\eta), 0,0\}$ & $\{0,0,(1-\eta)\}$ & constant \\
\hline 2 & $\psi(1-\xi)(1-\eta)$ & $\{\xi(1-\eta), 0,0\}$ & $\{0,0, \eta\}$ & - \\
\hline 3 & $\psi \xi(1-\eta)$ & $\{(1-\xi) \eta, 0,0\}$ & $\{0,(1-\xi), 0\}$ & - \\
\hline 4 & $(1-\psi) \xi(1-\eta)$ & $\{\xi \eta, 0,0\}$ & $\{0, \xi, 0\}$ & - \\
\hline 5 & $(1-\psi)(1-\xi) \eta$ & $\{0,(1-\psi)(1-\eta), 0\}$ & $\{(1-\psi), 0,0\}$ & - \\
\hline 6 & $\psi(1-\xi) \eta$ & $\{0, \psi(1-\eta), 0\}$ & $\{\psi, 0,0\}$ & - \\
\hline 7 & $\psi \xi \eta$ & $\{0,(1-\psi) \eta, 0\}$ & - & - \\
\hline 8 & $(1-\psi) \xi \eta$ & $\{0, \psi \eta, 0\}$ & - & - \\
\hline 9 & - & $\{0,0(1-\psi)(1-\xi)\}$ & - & - \\
\hline 10 & - & $\{0,0, \psi(1-\xi)\}$ & - & - \\
\hline 11 & - & $\{0,0,(1-\psi) \xi\}$ & - & - \\
\hline 12 & - & $\{0,0, \psi \xi\}$ & - & \\
\hline
\end{tabular}

\section{0-form basis}

The eight local 0-form basis functions referred to as the nodal basis functions are shown in Figure 3.12. For a mesh with $N_{\text {vols }}$ hexahedrons containing $N_{\text {nodes }}$ nodes 

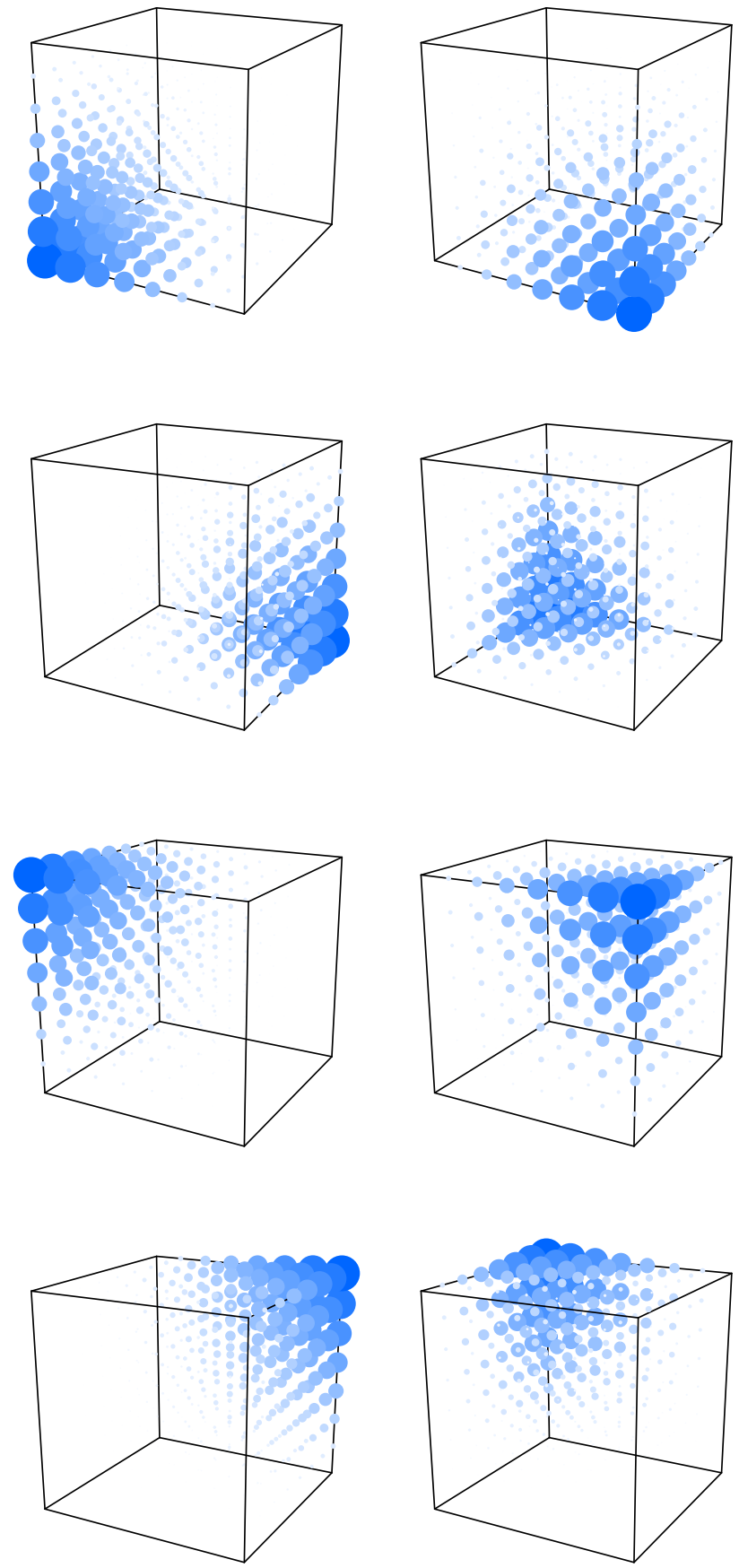

Figure 3.12: Hexahedral Node Basis Functions 
there is a linear nodal finite element $\left(\mathcal{K}_{i}, \mathcal{P}_{i}, \mathcal{A}_{i}\right)$ associated with each element. An arbitrary function $f$ can be expanded in $N_{\text {nodes }}$ global basis functions consisting of the local basis functions listed in the first column of Table 3.4 inside the element and zero outside. The collection of all of these global basis functions is referred to as $\mathcal{N}^{h}$ with dimension $N_{\text {nodes }}$. The value of $f$ is determined by the four values at the four nodes. Because the nodes are shared between adjoining elements the value of $f$ must be consistent giving a continuous function. The rate of convergence for this finite element is (3.46).

$$
\left|f-I_{T} f\right|_{2} \leq C h^{2}|f|_{\mathcal{H}^{2}}
$$

\section{1-form basis}

The twelve local 1-form basis functions referred to as the edge basis functions are shown in Figure 3.13. For a mesh with $N_{\text {vols }}$ hexahedrons containing $N_{\text {edges }}$ edges there is a linear edge finite element $\left(\mathcal{K}_{i}, \mathcal{P}_{i}, \mathcal{A}_{i}\right)$ associated with each element. An arbitrary function $f$ can be expanded in $N_{\text {edges }}$ global basis functions consisting of the local basis functions listed in the second column of Table 3.4 inside the element and zero outside. The collection of all of these global basis functions is referred to as $\mathcal{W}^{h}$ with dimension $N_{\text {edges }}$. The value of $f$ is determined by the six values at the six edges. The edge basis functions maintain tangential continuity by construction. The rate of convergence for this finite element is (3.47).

$$
\left|f-I_{T} f\right|_{\mathcal{H}(\text { curl })} \leq C h^{1}|f|_{\mathcal{H}^{1}}
$$



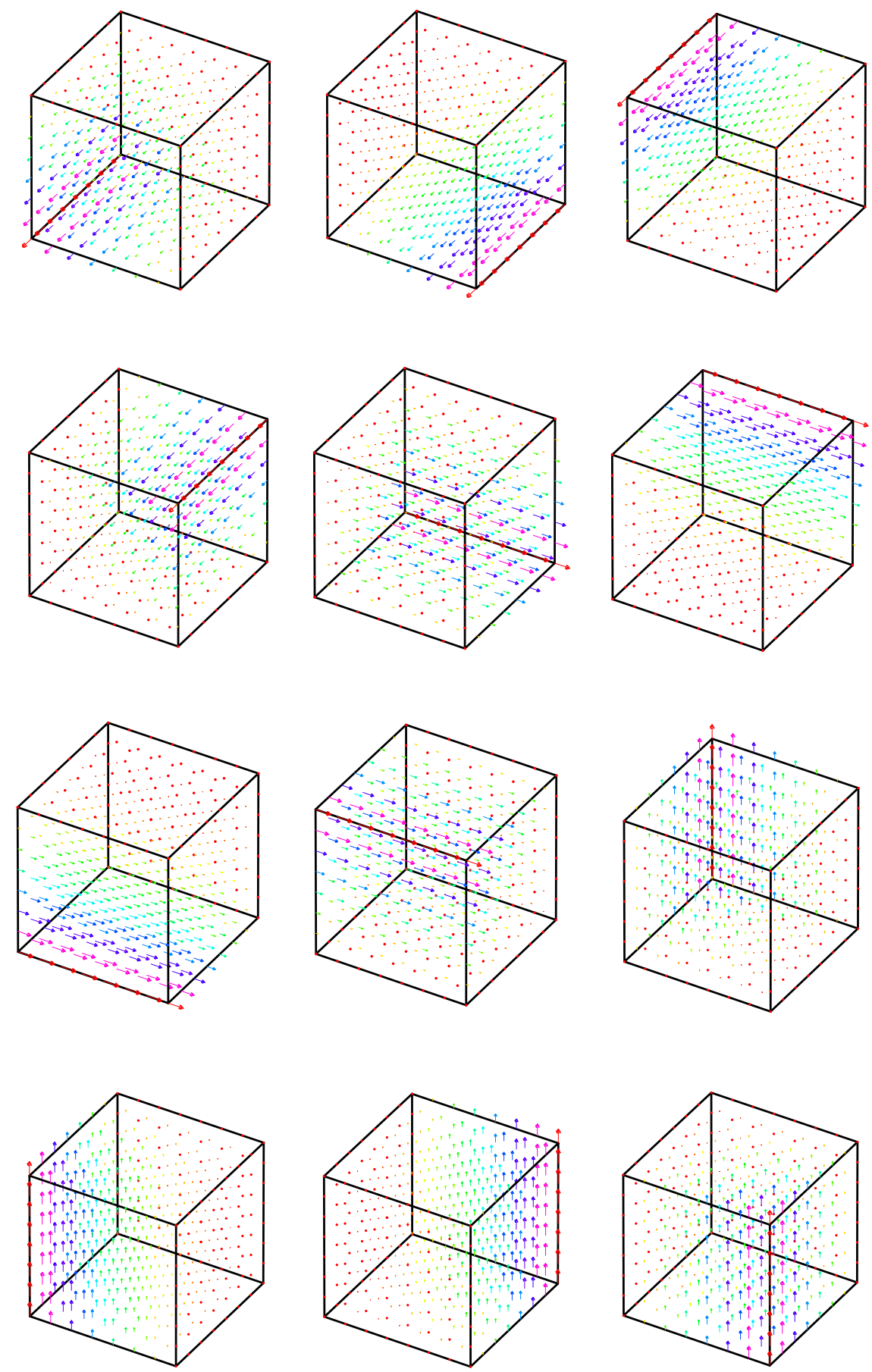

Figure 3.13: Hexahedral Edge Basis Functions 


\section{2-form basis}

The six local 2-form basis functions referred to as the face basis functions are shown in Figure 3.14. For a mesh with $N_{\text {vols }}$ hexahedrons containing $N_{\text {faces }}$ faces there is a linear face finite element $\left(\mathcal{K}_{i}, \mathcal{P}_{i}, \mathcal{A}_{i}\right)$ associated with each element. An arbitrary function $f$ can be expanded in $N_{\text {faces }}$ global basis functions consisting of the local basis functions listed in the third column of Table 3.4 inside the element and zero outside. The collection of all of these global basis functions is referred to as $\mathcal{F}^{h}$ with dimension $N_{\text {faces }}$. The value of $f$ is determined by the four values at the four faces. The face basis functions maintain normal continuity by construction. The rate of convergence for this finite element is (3.48).

$$
\left|f-I_{T} f\right|_{\mathcal{H}(\text { div })} \leq C h^{1}|f|_{\mathcal{H}^{1}}
$$

\section{3-form basis}

The single constant local 3-form basis functions referred to as the volume basis function is shown in Figure 3.15. For a mesh with $N_{\text {vols }}$ hexahedrons there is a linear face finite element $\left(\mathcal{K}_{i}, \mathcal{P}_{i}, \mathcal{A}_{i}\right)$ associated with each element. An arbitrary function $f$ can be expanded in $N_{\text {vols }}$ global basis functions consisting of the local basis functions listed in the fourth column of Table 3.4 inside the element and zero outside. The collection of all of these global basis functions is referred to as $\mathcal{S}^{h}$ with dimension $N_{\text {vols }}$. The value of $f$ is determined by the four values at the four faces. The volume basis functions have no continuity. The rate of convergence for this finite element is $(3.49)$.

$$
\left|f-I_{T} f\right|_{2} \leq C h^{1}|f|_{\mathcal{H}^{1}}
$$



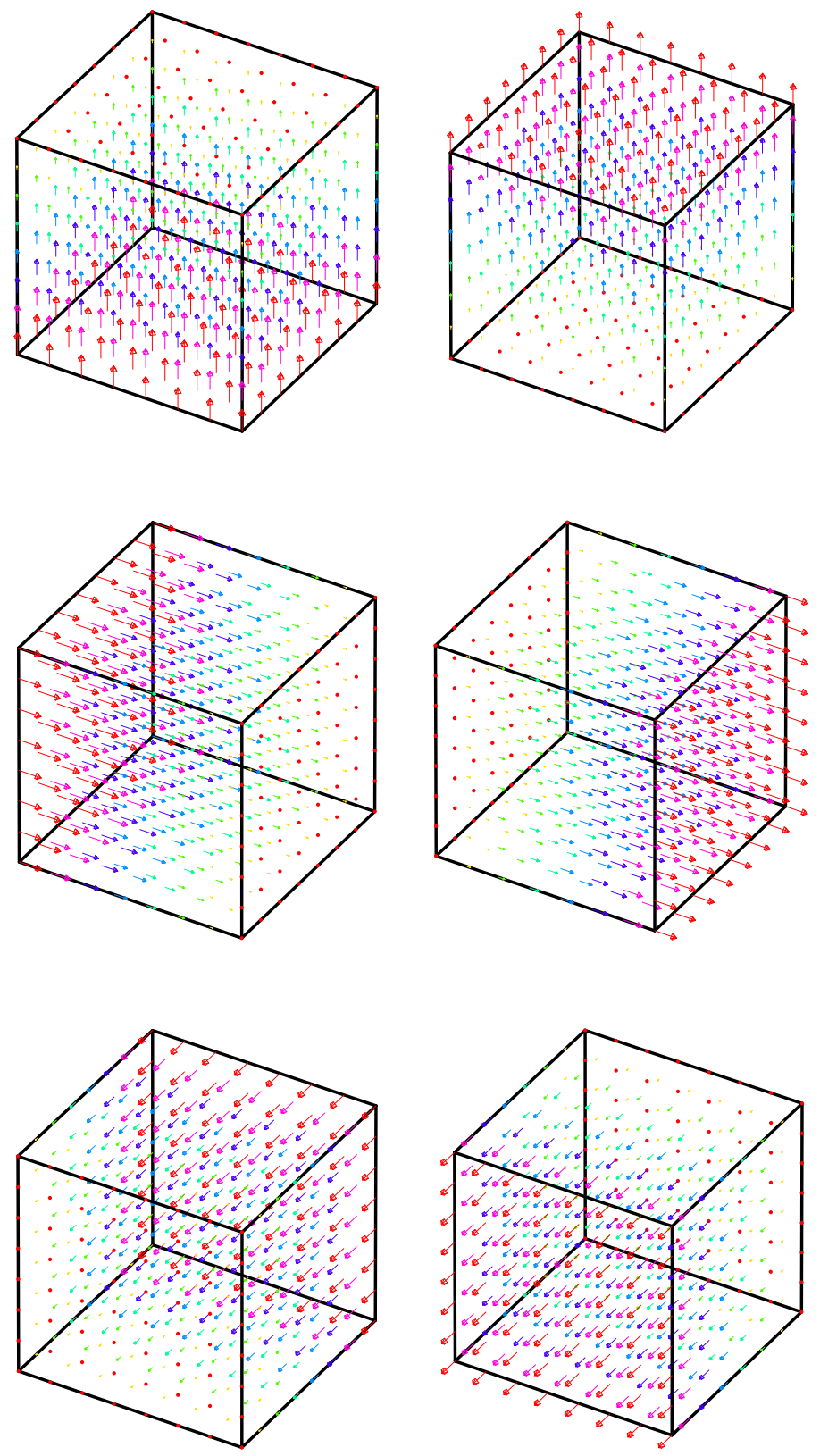

Figure 3.14: Hexahedral Face Basis Functions 


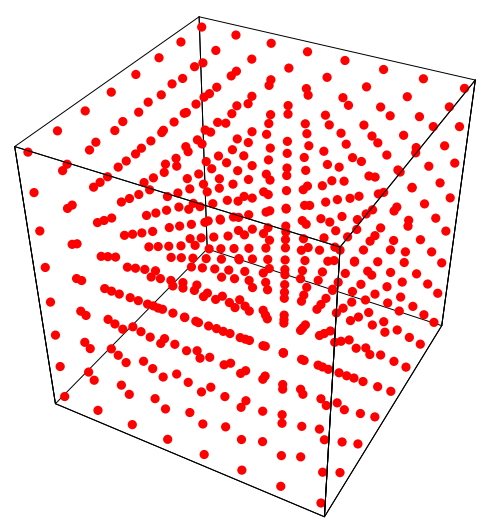

Figure 3.15: Hexahedral Volume Basis Functions

\subsubsection{Bilinear forms}

In the Galerkin finite element procedure bi-linear forms are required. These are easily generated from the general second-order equation (2.84) by taking the wedge product with an $(l-1)$-form $v$ and integrating over the volume $\Omega$,

$$
\int_{\Omega}(-1)^{l} d \star_{\alpha} d u \wedge v=-\int_{\Omega} \star_{\gamma} u \wedge v+\int_{\Omega} \Phi \wedge v
$$

Using the integration-by-parts formula

$$
\int_{\Omega} d \omega \wedge \eta+(-1)^{l} \int_{\Omega} \omega \wedge d \eta=\int_{\partial \Omega} \omega \wedge \eta
$$

yields the two key symmetric bilinear forms

$$
\begin{gathered}
a(u, v)=\int_{\Omega} \star_{\alpha}(d u) \wedge d v, \\
b(u, v)=\int_{\Omega} \star_{\gamma} u \wedge v .
\end{gathered}
$$

On the discrete level these bilinear forms are sums of integrals over each polyhedral domain $\mathcal{K}$. The continuous bilinear forms in (3.52) and (3.53) become (3.54) and (3.58) when the discrete differential forms are entered. The final integral in each 
case is over the reference element polyhedra $\tilde{\mathcal{K}}$ for the physical polyhedra $\mathcal{K}$. The isoparametric mapping $\mathcal{K}=\Phi(\tilde{\mathcal{K}})$ was shown previously in Table 2.3, the discrete versions are shown below.

$$
\begin{aligned}
a(u, v) & =\int_{\Omega} \star_{\alpha}(d u) \wedge d v \\
& =\sum_{\mathcal{K} \in T} \int_{\mathcal{K}=\Phi(\tilde{\mathcal{K}})} \star_{\alpha}(d u) \wedge d v \\
& =\sum_{\mathcal{K} \in T} \int_{\tilde{\mathcal{K}}} \Phi^{\star}\left(\star_{\alpha}(d u) \wedge d v\right)|\Phi| \\
& =\sum_{\mathcal{K} \in T} \int_{\tilde{\mathcal{K}}} \star_{\alpha \cdot \Phi}\left(\Phi^{*}(d u)\right) \wedge \Phi^{*}(d v)|\Phi| . \\
b(u, v) & =\sum_{\mathcal{K} \in T} \int_{\tilde{\mathcal{K}}} \star\left(\Phi^{*}(u)\right) \wedge \Phi^{*}(v)|\Phi| .
\end{aligned}
$$

These bilinear forms are utilized in the sections below to define the finite element method for the various $p$-forms.

\subsubsection{Discrete differential operators}

In this section we will discuss the various scalar and vector differential operators and their transformation from the reference to physical frames. The natural and adjoint continuous differential operators were presented previously in Table 2.4. The corresponding discrete versions are given in Table 3.5.

The matrices presented in Table 3.5 are constructed using the bilinear forms and basis functions presented in the sections above. The mass matrices, $\mathbf{M}_{p}^{\gamma}$, for each of the $p$-forms with scalar, $\alpha$ and tensor, $\beta$ materials $\gamma$ are listed in (3.59).

$$
\begin{gathered}
\left(\mathbf{M}_{0}^{\alpha}\right)_{i, j}=\left(\alpha N_{i}, N_{j}\right) ; N_{i}, N_{j} \in \mathcal{N}^{h} \\
\left(\mathbf{M}_{1}^{\beta}\right)_{i, j}=\left(\beta \vec{W}_{i}, \vec{W}_{j}\right) ; \vec{W}_{i}, \vec{W}_{j} \in \mathcal{W}^{h}
\end{gathered}
$$


Table 3.5: Discrete Differential Operators

\begin{tabular}{|c|c|c|c|c|c|}
\hline \multicolumn{6}{|c|}{ Discrete Differential Operators } \\
\hline \hline \multirow{2}{*}{} & \multicolumn{4}{|c|}{ Domain } \\
\cline { 2 - 6 } \multicolumn{1}{|c||}{} & $\mathcal{N}^{h}$ & $\mathcal{W}^{h}$ & $\mathcal{F}^{h}$ & $\mathcal{S}^{h}$ \\
\hline \multirow{3}{*}{ Range } & $\mathcal{N}^{h}$ & & $\mathbf{D}$ & & \\
\cline { 2 - 6 } & $\mathcal{W}^{h}$ & $\mathbf{G}$ & & $\tilde{\mathbf{C}}$ & \\
\cline { 2 - 6 } & $\mathcal{F}^{h}$ & & $\mathbf{C}$ & & $\mathbf{G}$ \\
\cline { 2 - 6 } & $\mathcal{S}^{h}$ & & & $\mathbf{D}$ & \\
\hline
\end{tabular}

$$
\begin{aligned}
& \left(\mathbf{M}_{2}^{\beta}\right)_{i, j}=\left(\beta \vec{F}_{i}, \vec{F}_{j}\right) ; \vec{F}_{i}, \vec{F}_{j} \in \mathcal{F}^{h} \\
& \left(\mathbf{M}_{3}^{\alpha}\right)_{i, j}=\left(\alpha S_{i}, S_{j}\right) ; S_{i}, S_{j} \in \mathcal{S}^{h}
\end{aligned}
$$

The units for the $p$-form mass matrices are $\left[m^{3}\right] \cdot[\alpha],[m] \cdot[\beta],\left[\frac{1}{m}\right] \cdot[\beta]$ and $\left[\frac{1}{m^{3}}\right] \cdot[\alpha]$ for the $p=0,1,2,3$ forms respectively. The values $[\alpha]$ and $[\beta]$ are the units of the scalar and tensor materials respectively. The stiffness matrices, $\mathbf{K}_{p}^{\gamma}$, associated with the bilinear form in (3.52) with scalar and tensor materials $\gamma$ represented by $\alpha$ and $\beta$ respectively are listed in (3.60).

$$
\begin{aligned}
\left(\mathbf{K}_{0}^{\beta}\right)_{i, j} & =\left(\beta \nabla N_{i}, \nabla N_{j}\right) \\
\left(\mathbf{K}_{1}^{\beta}\right)_{i, j} & =\left(\beta \nabla \times \vec{W}_{i}, \nabla \times \vec{W}_{j}\right) \\
\left(\mathbf{K}_{2}^{\alpha}\right)_{i, j} & =\left(\alpha \nabla \cdot \vec{F}_{i}, \nabla \cdot \vec{F}_{j}\right)
\end{aligned}
$$

The units of the stiffness matrices are $[m] \cdot[\beta],\left[\frac{1}{m}\right] \cdot[\beta]$ and $\left[\frac{1}{m^{3}}\right] \cdot[\alpha]$ for the $p=0,1,2$ forms respectively.

The matrices $\mathbf{G}, \mathbf{C}$ and $\mathbf{D}$ are also referred to as incident matrices and play a crucial role in conservation. The discrete Grad, Div and Curl matrices represent the discrete version (3.61) of the continuous inclusion property (2.77).

$$
\nabla \mathcal{N}^{h} \in \mathcal{W}^{h}
$$




$$
\begin{array}{r}
\nabla \times \mathcal{W}^{h} \in \mathcal{F}^{h} \\
\nabla \cdot \mathcal{F}^{h} \in \mathcal{S}^{h}
\end{array}
$$

These inclusion relations allow the matrices $\mathbf{G}, \mathbf{C}$ and $\mathbf{D}$ to be written in terms of the connectivity of the grid and do not depend on the coordinates of the grid. The discrete Grad matrix has integer coefficients for the linear basis functions that depend on the direction of the edge to which the two nodes are connected. The discrete Curl and Div matrix also have integer coefficients for the linear basis functions with similar dependencies.

The matrices that form the discrete differential natural operators for the gradient $\mathbf{G}$, curl $\mathbf{C}$ and divergence $\mathbf{D}$ can be constructed by the coefficients formed by the inclusion relation $(3.62)$.

$$
\begin{aligned}
\nabla N_{i} & =\sum_{j} g_{i, j} \vec{W}_{j} \\
\nabla \times \vec{W}_{i} & =\sum_{j} c_{i, j} \vec{F}_{j} \\
\nabla \cdot \vec{F}_{i} & =\sum_{j} d_{i, j} S_{j}
\end{aligned}
$$

The coefficients $g_{i, j}$ for the linear basis functions will be nonzero for a node $i$ if this node is attached to edge $j$. The coefficients $c_{i, j}$ will be nonzero if the edge $i$ is a member of the the face $j$. The coefficients of $d_{i, j}$ will be nonzero if the face $i$ is a member of element $j$. The discrete differential operators are listed in (3.63).

$$
\begin{aligned}
\mathbf{G}_{i, j} & =g_{i, j} \\
\mathbf{C}_{i, j} & =c_{i, j} \\
\mathbf{D}_{i, j} & =d_{i, j}
\end{aligned}
$$


The matrices that form the discrete differential adjoint operators for the gradient $\tilde{\mathbf{G}}$, curl $\tilde{\mathbf{C}}$ and divergence $\tilde{\mathbf{D}}$ are listed in (3.64).

$$
\begin{aligned}
\tilde{\mathbf{D}} & =\mathbf{M}_{0}^{-1} \mathbf{G}^{T} \mathbf{M}_{1} \\
\tilde{\mathbf{C}} & =\mathbf{M}_{1}^{-1} \mathbf{C}^{T} \mathbf{M}_{2} \\
\tilde{\mathbf{G}} & =\mathbf{M}_{2}^{-1} \mathbf{D}^{T} \mathbf{M}_{3}
\end{aligned}
$$

Having satisfied this inclusion property, the discrete differential forms now satisfy the discrete exact sequence property and the commuting property.

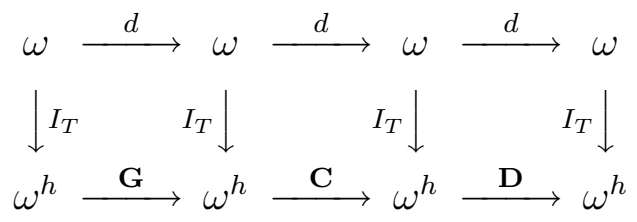

The discrete differential operators listed above preserve the continuous property of the exact sequence on the discrete level by defining the spaces used to represent the forms and operators on the discrete level so that they maintain the same sequence as in the continuous sequence.

The adjoint discrete differential forms have the same sequence as the continuous forms.

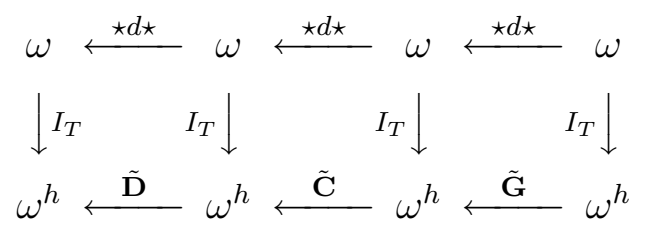

In this operator sequence the role of the mass matrix is the discrete Hodge star operator. This is discussed in more depth in [41]. 
Combining the natural operators and adjoint operators the discrete version of the vector identities shown in (2.78) can be constructed for the natural (3.65) and adjoint $(3.66)$.

$$
\begin{aligned}
& \mathbf{C G}=0 \\
& \mathbf{D C}=0 \\
& \tilde{\mathbf{C}} \tilde{\mathbf{G}}=0 \\
& \tilde{\mathbf{D}} \tilde{\mathbf{C}}=0
\end{aligned}
$$

\subsubsection{Basis transformations}

Defining the Jacobian as the transformation from the reference to the physical frame in $R^{3}$

$$
\mathbf{J}=\frac{\partial\left(x_{1}, x_{2}, x_{3}\right)}{\partial\left(\psi_{1}, \psi_{2}, \psi_{3}\right)}
$$

we can calculate the operators in the reference coordinates and project them, using the Jacobian, to the physical coordinates.

\section{Scalar gradient}

If we define the gradient in the physical coordinates as $\nabla \phi$ for an arbitrary scalar function $\phi$ and the gradient operator in the reference coordinates as $\bar{\nabla} \phi$ then these two operators are related by the Jacobian and transform as shown in (3.68)

$$
\nabla \phi=\mathbf{J}^{-1} \bar{\nabla} \phi
$$

This operation can be defined by looking at the gradient in the reference coordinates and using the chain rule to determine the gradient in the physical coordinates. 
The component description of the gradient in the reference coordinates is shown in (3.69).

$$
(\bar{\nabla})^{i}=\frac{\partial}{\partial \psi_{i}}
$$

Using the chain rule these components can be written in terms of the physical coordinates (3.70) for the gradient operating on a scalar function $\phi$

$$
\frac{\partial \phi}{\partial \psi_{i}}=\frac{\partial x_{i}}{\partial \psi_{i}} \frac{\partial \phi}{\partial x_{i}}
$$

where $\frac{\partial}{\partial x_{i}}=(\nabla)^{i}$. Inverting the relation gives the transformation shown in (3.68). The gradient is the natural differential operator for the 0 -forms. The transformation of the gradient of a reference 0 -form basis function to the physical 0-form basis function is shown in (3.71).

$$
\nabla N_{i}=\mathbf{J}^{-1} \bar{\nabla} \bar{N}_{i}
$$

\section{Curl}

As discussed in appendix $\mathrm{A}$ of [42], the curl of a vector $\vec{U}$ transforms from the physical coordinate system $\nabla \times \vec{U}$ to the reference coordinates $\bar{\nabla} \times \overline{\vec{U}}$ as shown in (3.72).

$$
\nabla \times \vec{U}=\frac{\mathbf{J}^{T}}{|\mathbf{J}|} \bar{\nabla} \times \overline{\vec{U}}
$$

The curl is the natural differential operator for the 1-forms. The transformation of the curl of a reference 1-form basis function to the physical 1-form basis function is shown in (3.73).

$$
\nabla \times \vec{W}_{i}=\frac{\mathbf{J}^{T}}{|\mathbf{J}|} \bar{\nabla} \times \overline{\vec{W}}_{i}
$$


In addition to the transformations of the operators, due to their vector nature, the vector 1-form and 2-form basis functions must also be transformed from the reference to physical coordinates.

\section{1-form basis functions}

The inclusion relation $\nabla N_{i} \in\left\{\mathcal{W}^{h}\right\}$ shows the 1 -form basis functions are in the same space as the gradient of the 0 -form basis functions. The gradient of a 0 -form basis function can be written as a sum of 1-form basis functions. Due to this fact the 1-forms must transform in the same way as the gradient of the 0-form basis functions. This transformation is called a covariant transformation and transforms the reference basis function $\overline{\vec{W}}_{i}$ to the physical basis function $\vec{W}_{i}$.

$$
\vec{W}_{i}=\mathbf{J}^{-1} \overline{\vec{W}}_{i}
$$

\section{2-form basis functions}

The inclusion relation $\nabla \times \vec{W}_{i} \in\left\{\mathcal{F}^{h}\right\}$ shows the 2 -form basis functions is in the same space as the 1-form basis functions. The curl of a 1-form basis function can be written as a sum of 2-form basis functions. Due to this fact the 2-form basis function must transform in the same way as the curl of a 1-form basis function. This transformation is called a contravariant transformation from the reference basis function $\overrightarrow{\vec{F}}_{i}$ to the physical basis function $\vec{F}_{i}$.

$$
\vec{F}_{i}=\frac{\mathbf{J}^{T}}{|\mathbf{J}|} \overline{\vec{F}}_{i}
$$




\section{Divergence}

The divergence, being a scalar function, does not need a transformation from the reference to physical coordinates. But in order to match the units imposed on the 2 -form basis function transformation, the reference element divergence $\bar{\nabla} \cdot$ must be divided by the determinant of the Jacobian when a transformation to the physical coordinates takes place $(3.76)$

$$
\nabla \cdot=\frac{1}{|\mathbf{J}|} \bar{\nabla}
$$

\subsection{Properties of the Discrete $p$-forms}

The tables for the $p$-form properties Table 1.1 and Table 2.2 can now be extended with the properties discussed in the above sections giving Table 3.6.

Table 3.6: Properties of the $p$-forms

\begin{tabular}{|c|c|c|c|c|}
\hline Property & 0-form & 1-form & 2-form & 3-form \\
\hline \hline Minimum Continuity & Total & Tangential & Normal & None \\
\hline Integral & Point & Line & Surface & Volume \\
\hline Derivative & Grad & Div & Curl & None \\
\hline $\begin{array}{c}\text { Physical } \\
\text { Type }\end{array}$ & Potentials & Fields & $\begin{array}{c}\text { Fluxes, } \\
\text { Vector Densities }\end{array}$ & $\begin{array}{c}\text { Scalar } \\
\text { Densities }\end{array}$ \\
\hline Hilbert Space & $\mathcal{H}(g r a d)$ & $\mathcal{H}($ div $)$ & $\mathcal{H}($ curl $)$ & $L^{2}$ \\
\hline Basis Function & $\mathcal{N}$ & $\mathcal{W}$ & $\mathcal{F}$ & $\mathcal{S}$ \\
\hline Discrete Derivative & $\mathbf{G}$ & $\mathbf{C}$ & $\mathbf{D}^{-1}$ & None \\
\hline $\begin{array}{c}\text { Adjoint Discrete } \\
\text { Derivative }\end{array}$ & None & $\mathbf{M}_{0}^{-1} \mathbf{G}^{T} \mathbf{M}_{1}$ & $\mathbf{M}_{1}^{-1} \mathbf{M}_{2}$ & $\mathbf{M}_{2}^{-1} \mathbf{D}^{T} \mathbf{M}_{3}$ \\
\hline $\begin{array}{c}\text { Discrete } \\
\text { PushForward }\end{array}$ & 1 & $\mathbf{J}^{-1}$ & $\frac{1}{|\mathbf{J}|} \mathbf{J}^{T}$ & None $^{\text {Discrete }}$ \\
$\begin{array}{c}\text { PullBack } \\
\mathbf{J}^{-1}\end{array}$ & $\frac{1}{|\mathbf{J}|} \mathbf{J}^{T}$ & $\frac{1}{|\mathbf{J}|}$ & None \\
\hline
\end{tabular}




\subsection{Spurious Modes}

Spurious or non-physical modes arise in discrete simulations when the wrong discrete $p$-form has been chosen to represent the physical variable. In electrodynamics simulations the electric and magnetic fields are continuous in homogeneous regions. A discrete simulation might therefore use three totally continuous nodal basis functions to represent the three components of the fields. If the nodal basis functions are chosen to represent the 1-form electric field for the equation (3.77) spurious modes will arise.

$$
\nabla \times \mu^{-1} \nabla \times \vec{E}=k^{2} \epsilon \vec{E}
$$

The reason spurious modes arise when nodal basis functions are used to describe the electric field is in the choice of space [36]. Let $\overrightarrow{\mathcal{N}}^{h}$ be the vector set of totally continuous nodal basis functions defined above. Using this space it is not possible, in general, to represent a divergence free field (3.78).

$$
\{\vec{v} \in \overrightarrow{\mathcal{N}}: \nabla \cdot \vec{v}=0\}=\{\emptyset\}
$$

In [43] the spurious modes are shown to be caused by the choice of the vector nodal basis functions. If the divergence of equation (3.77) is performed the resulting divergence equation (3.79) will only be satisfied if $k^{2}=0$ or $\nabla \cdot \epsilon \vec{E}=0$.

$$
k^{2} \nabla \cdot \epsilon \vec{E}=0
$$

It was shown above that a divergence-free electric flux density cannot be constructed using vector nodal basis functions. A field with zero divergence is called a non-static solution to (3.77). Fields with $k^{2}=0$ are called static solutions and can be described 
by the gradient of the scalar potential $\vec{E}=-\nabla \phi$. If nodal basis functions are used to represent $\vec{E}$ then it is not possible to satisfy $\nabla \times \nabla \phi=0$, therefore the eigenvalues $k^{2}$ are non-zero giving rise to spurious modes. The vector nodal basis functions provide a poor null space for the $\nabla \times \nabla \times$ operator resulting in spurious modes. The problem was overcome by selecting basis functions that include the gradients of the scalar potentials. The inclusion relations given above showed that $\nabla \mathcal{N}^{h} \in \mathcal{W}^{h}$ so the correct basis functions to use for the electric field are the 1-form edge basis functions. The discrete differential forms framework provides this information at the outset and the resulting operator provides the correct nullspace for the $\nabla \times \nabla \times$ operator.

The acoustic wave equation in the frequency domain (3.80) will have a similar problem if the vector nodal basis functions are chosen.

$$
\nabla \nabla \cdot \vec{v}=-k^{2} \vec{v}
$$

Taking the curl of (3.80) results in the equation (3.81).

$$
-k^{2} \nabla \times \vec{v}=0
$$

This equation will be satisfied if $k=0$ or $\nabla \times \vec{v}=0$. Unfortunately the vector nodal basis functions cannot, in general, represent a curl free field (3.82) any more than they can represent a divergence free field.

$$
\{\vec{v} \in \overrightarrow{\mathcal{N}}: \nabla \times \vec{v}=0\}=\{\emptyset\}
$$

It was shown above that a vector variable represented by nodal basis functions cannot represent a divergence free field. In this case the nodal basis functions provide a poor approximation to the null space of the $\nabla \nabla \cdot$ operator. If $\nabla \nabla \cdot \vec{v}$ is not zero then $k^{2}$ 
cannot be zero, resulting in spurious modes. The solution is again to use the proper basis functions to represent $\vec{v}$. In this case the proper basis functions are the 2 -form face basis functions.

\subsection{Examples}

Solving partial differential equations becomes an exercise in combining the previous sections' operators. In this section we will show examples from many different fields including Electromagnetics, Linear Acoustics, and Linear Elasticity. The properties for the various Differential Forms listed in Table 3.6 make the choice of basis functions for each variable obvious.

\subsubsection{Scalar wave equations}

The variational form for the second order scalar wave equation for $\phi$ which will represent the velocity potential, density or the pressure is given in (3.83)

$$
\frac{\partial^{2}}{\partial t^{2}}\left(\phi, \phi^{*}\right)=-c_{l}^{2}\left(\nabla \phi, \nabla \phi^{*}\right)-c_{l}^{2} \oint_{\Gamma} \phi^{*} \nabla \phi \cdot \hat{n} \forall \phi^{*}
$$

where $\phi \in \mathcal{H}(\operatorname{grad})$ and $\phi^{*} \in \mathcal{H}_{0}(\operatorname{grad})$. Due to the test space used for test variable $\phi^{*}$ the boundary integral term on the right hand side is zero. This variational form also presents the valid boundary conditions for this equation. The variable itself can be specified on the boundary resulting in the Dirichlet boundary condition $\phi=D$ on $\Gamma_{D}$ or the normal component of the gradient can be specified resulting in the Neumann boundary condition $\nabla \phi \cdot \hat{n}=N$ on $\Gamma_{N}$.

For the natural form of the scalar wave equation the variable $\phi$ is expanded in 
terms of the nodal basis functions resulting in a 0 -form (3.84).

$$
\phi=\sum_{i=1}^{N_{\text {nodes }}} \alpha_{i} N_{i}
$$

Entering this expansion into the variational form (3.83) results in the explicit integrodifferential form $(3.85)$

$$
\int_{\Omega} \nabla \cdot \nabla \phi N_{j}=-\int_{\Omega} \nabla \phi \cdot \nabla N_{j}+\oint_{\Gamma} N_{j} \nabla \phi \cdot \hat{n} \quad \forall \quad N_{j}
$$

with boundary conditions

$$
\begin{array}{r}
\phi=D \text { on } \Gamma_{D}, \text { essential } \\
\nabla \phi \cdot \hat{n}=N \text { on } \Gamma_{N}, \text { natural }
\end{array}
$$

where $\Gamma_{D}$ and $\Gamma_{N}$ denote the boundaries for Dirichlet and Neumann boundary conditions respectively and the essential and natural properties refer to the boundary conditions that are automatically enforced with no matrix manipulation (natural) and those that must be enforced by matrix manipulation (essential). The discrete form of $(3.85)$ is shown in $(3.87)$

$$
\mathbf{M}_{0} \frac{\partial^{2} \vec{\beta}}{\partial t^{2}}=-\mathbf{K}_{0}^{c_{l}^{2}} \vec{\beta}
$$

The non-adjoint problem can also be written in mixed finite element form using the variational method for the first order scalar wave equations for pressure $P$ and velocity $\vec{v}$.

$$
\begin{array}{r}
\frac{\partial}{\partial t}\left(P, P^{*}\right)=\left(\gamma P_{0}\right)\left(\vec{v}, \nabla P^{*}\right)-\left(\gamma P_{0}\right) \oint_{\Gamma} P^{*} \vec{v} \cdot \hat{n} \forall P^{*} \\
\frac{\partial v_{1}}{\partial t}\left(\vec{v}, \vec{v}^{*}\right)=-\frac{1}{\rho_{0}}\left(\nabla P, \vec{v}^{*}\right) \forall \vec{v}^{*}
\end{array}
$$


where $P \in \mathcal{H}($ grad $), P^{*} \in \mathcal{H}_{0}($ grad $), \vec{v} \in \mathcal{H}($ curl $)$ and $\vec{v}^{*} \in \mathcal{H}_{0}($ curl $)$. In this case the pressure is expanded as in (3.84), but the velocity is expanded as in (3.89).

$$
\vec{v}=\sum_{j=1}^{N_{\text {edges }}} \beta_{j} \vec{W}_{j}
$$

Using a curl-conforming space for a divergence conforming variable would seem to be nonconforming with respect to the equations at first glance. But it will be shown that the resulting discrete form of this solution is exactly the same as (3.87). The resulting discrete form of the equations in (3.88) are listed in (3.90)

$$
\begin{array}{r}
\mathbf{M}_{0} \ddot{\vec{\alpha}}=-\mathbf{G}^{T} \mathbf{M}_{1} \dot{\vec{\beta}}+\oint_{\Gamma} N_{l} \dot{\vec{v}} \cdot \hat{n} \\
\mathbf{M}_{1} \dot{\vec{\beta}}=\mathbf{M}_{1} \mathbf{G} \vec{\alpha}
\end{array}
$$

where the boundary condition matrix in this case is:

$$
\oint_{\Gamma} N_{l} \vec{u} \cdot \vec{n}=\mathrm{GB}_{1} \vec{\beta}=0, N_{l} \in \mathcal{H}_{0}(\operatorname{grad}, \bar{\Omega})
$$

The mixed matrix form for the second order equations is represented in (3.92).

$$
\left[\begin{array}{cc}
\mathbf{M}_{1} & -\mathbf{M}_{1} \mathbf{G} \\
-\mathbf{G}^{T} \mathbf{M}_{1} & 0
\end{array}\right]\left[\begin{array}{c}
\dot{\vec{\beta}} \\
\vec{\alpha}
\end{array}\right]=\left[\begin{array}{c}
0 \\
\mathbf{M}_{0} \ddot{\vec{\alpha}}
\end{array}\right]
$$

Rewriting this in single equation form (3.93) results in the matrix equation given in $(3.87)$.

$$
\mathbf{M}_{0} \frac{\partial^{2} \vec{\beta}}{\partial t^{2}}=-c_{l}^{2} \mathbf{G}^{T} \mathbf{M}_{\mathbf{1}} \mathbf{G} \vec{\beta}=-\mathbf{K}_{0}^{c_{l}^{2}} \vec{\beta}
$$

An alternate method is to use the adjoint form of the Laplacian. In this form the pressure $P$ is discretized as a piecewise discontinuous 3 -form using the volume basis functions (3.94) and the velocity is discretized as a 2-form (3.95).

$$
P=\sum_{i=1}^{N_{\text {elements }}} \alpha_{i} S_{i}
$$




$$
\vec{v}=\sum_{i=1}^{N_{\text {faces }}} \beta_{i} \vec{F}_{i}
$$

The variational form is given in (3.96)

$$
\begin{array}{r}
\frac{\partial}{\partial t}\left(P, P^{*}\right)=-\left(\gamma P_{0}\right)\left(\nabla \cdot \vec{v}, P^{*}\right) \forall P^{*} \\
\frac{\partial v_{1}}{\partial t}\left(\vec{v}, \vec{v}^{*}\right)=\frac{1}{\rho_{0}}\left(P, \nabla \cdot \vec{v}^{*}\right)-\frac{1}{\rho_{0}} \oint_{\Gamma} P \vec{v}^{*} \cdot \hat{n} \forall \vec{v}^{*}
\end{array}
$$

with the new solution and test spaces spaces for the variables given by $P \in L^{2}$, $P^{*} \in L^{2}, \vec{v} \in \mathcal{H}($ div $)$ and $\vec{v}^{*} \in \mathcal{H}_{0}($ div $)$.

The discrete form of the adjoint scalar wave equations is shown in (3.97).

$$
\begin{array}{r}
\mathbf{M}_{3} \dot{\vec{\alpha}}=\mathbf{M}_{3} \mathbf{D} \vec{\beta} \\
\mathbf{M}_{2} \dot{\vec{\beta}}=-\mathbf{D}^{T} \mathbf{M}_{3} \vec{\alpha}+\oint_{\Gamma} N_{i} \vec{v}^{*} \cdot \hat{n}
\end{array}
$$

The essential and natural boundary conditions are switched 0-form scalar wave equation and are given in (3.98). The essential and natural boundary conditions are ooposite from the previous wave equation.

$$
\begin{array}{r}
\nabla P \cdot \hat{n}=N \text { on } \Gamma_{N}, \text { essential } \\
P=D \text { on } \Gamma_{D}, \text { natural }
\end{array}
$$

The boundary matrix in this case is zero due to the choice of test space and is shown in (3.99).

$$
\oint_{\Gamma} P \vec{v}^{*} \cdot \hat{n}=\oint_{\Gamma} N_{i} \vec{F}_{j} \cdot \hat{n}=0, \vec{F}_{j} \in \mathcal{H}_{0}(\operatorname{div}, \bar{\Omega})
$$

In mixed second order matrix form the equations in (3.97) become (3.100).

$$
\left[\begin{array}{cc}
\mathbf{M}_{2} & \mathbf{D}^{T} \mathbf{M}_{3} \\
\mathbf{D} & 0
\end{array}\right]\left[\begin{array}{l}
\dot{\vec{\beta}} \\
\vec{\alpha}
\end{array}\right]=\left[\begin{array}{c}
0 \\
\ddot{\vec{\alpha}}
\end{array}\right]
$$


In symmetrized form (3.100) becomes (3.101).

$$
\left[\begin{array}{cc}
\mathbf{M}_{2} & \mathbf{D}^{T} \mathbf{M}_{3} \\
\mathbf{M}_{3} \mathbf{D} & 0
\end{array}\right]\left[\begin{array}{c}
\dot{\vec{\beta}} \\
\vec{\alpha}
\end{array}\right]=\left[\begin{array}{c}
0 \\
\mathbf{M}_{3} \ddot{\vec{\alpha}}
\end{array}\right]
$$

\subsubsection{Electromagnetic wave equations}

The variational form of the magnetic flux density wave equation will be derived from the first order equations listed in (2.27). The variational form of these equations using $\vec{B} \in \mathcal{H}($ div $), \vec{B}^{*} \in \mathcal{H}_{0}($ div $), \vec{E} \in \mathcal{H}($ curl $)$, and $\vec{E}^{*} \in \mathcal{H}_{0}($ curl $)$ results in the first order variational form (3.102) and (3.103).

$$
\begin{aligned}
& \frac{\partial}{\partial t}\left(\mu^{-1} \vec{B}, \vec{B}^{*}\right)=-\left(\mu^{-1} \nabla \times \vec{E}, \vec{B}^{*}\right)-\left(\mu^{-1} \sigma_{M} \mu^{-1} \vec{B}, \vec{B}^{*}\right)-\left(\mu^{-1} \vec{M}_{s}, \vec{B}^{*}\right) \forall \vec{B}^{*} \\
& \frac{\partial}{\partial t}\left(\epsilon \vec{E}, \vec{E}^{*}\right)=\left(\mu^{-1} \vec{B}, \nabla \times \vec{E}^{*}\right)-\left(\sigma_{E} \vec{E}, \vec{E}^{*}\right)-\left(\vec{J}, \vec{E}^{*}\right)+\oint_{\Gamma} \vec{E}^{*} \times \mu_{-1} \vec{B} \cdot \hat{n} \forall \vec{E}^{*}
\end{aligned}
$$

In this case the vector identity (B.3) is used to form (3.103). This integration is shown in (3.104).

$$
\int_{\Omega} \nabla \times \mu-1 \cdot \vec{E}^{*}=\int_{\Omega} \mu^{-1} \vec{B} \cdot \nabla \times \vec{E}^{*}+\int_{\Omega} \nabla \cdot\left(\vec{E}^{*} \times \mu^{-1} \vec{B}\right)
$$

The last term on the right hand side of (3.104) can be integrated using Gauss's law resulting in the boundary term (3.105).

$$
\int_{\Omega} \nabla \cdot\left(\vec{E}^{*} \times \mu^{-1} \vec{B}\right)=\oint_{\Gamma} \vec{E}^{*} \times \mu_{-1} \vec{B} \cdot \hat{n}
$$

For the magnetic flux density wave equation $\vec{B}, \vec{J}_{s}$, and $\vec{M}_{s}$ are all discretized in terms of 2-form face basis functions (3.106),(3.107), and (3.108),

$$
\vec{B}=\sum_{i=1}^{N_{\text {faces }}} b_{i} \vec{F}_{i}
$$




$$
\begin{aligned}
\vec{J} & =\sum_{i=1}^{N_{\text {faces }}} j_{i} \vec{F}_{i} \\
\vec{M} & =\sum_{i=1}^{N_{\text {faces }}} m_{i} \vec{F}_{i}
\end{aligned}
$$

For the electric field equation we will discretize $\vec{E}$ in terms of the 1-form edge basis functions $W_{i} \in \mathcal{W}^{h}(3.109)$.

$$
\vec{E}=\sum_{i=1}^{N_{\text {edges }}} e_{i} \vec{W}_{i}
$$

Entering the discrete form of the magnetic flux density along with the discrete form of the electric field given above results in the discrete first order Maxwell's equations (3.110) and (3.110).

$$
\begin{gathered}
\mathbf{M}_{2}^{\mu-1} \dot{\vec{b}}=-\mathbf{M}_{2}^{\mu^{-1}} \mathbf{C} \vec{e}-\mathbf{M}_{2}^{\left(\mu^{-1} \sigma_{M} \mu^{-1}\right)} \vec{b}-\mathbf{M}_{2}^{\mu^{-1}} \vec{m} \\
\mathbf{M}_{1}^{\epsilon} \dot{\vec{e}}=-\mathbf{C}^{T} \mathbf{M}_{2}^{\mu^{-1}} \vec{b}-\mathbf{M}_{1}^{\sigma_{E}} \vec{e}-\mathbf{X} \vec{j}-\oint_{\Gamma} \vec{W}_{j} \cdot \vec{B} \times \hat{n}
\end{gathered}
$$

The boundary conditions for this case are:

$$
\begin{aligned}
\vec{B} \cdot \hat{n} & =\vec{D} \text { on } \Gamma_{D} \\
\vec{B} \times \hat{n} & =\vec{N} \text { on } \Gamma_{N}
\end{aligned}
$$

The boundary condition matrix in this case evaluates to zero (3.113) due to the choice of test space for $\vec{E}^{*}$.

$$
\oint_{\Gamma} \vec{W}_{k} \cdot \vec{B} \times \hat{n}=\left(\vec{F}_{i}, \hat{n} \times \vec{W}_{j}\right)_{\Gamma}=0, \vec{W}_{j} \in H_{0}(\nabla \times, \bar{\Omega})
$$

Combining the first order equations in a zero source region and differentiating the magnetic flux density (3.110) results in the second order mixed discrete wave equation $(3.114)$

$$
\left[\begin{array}{cc}
\mathbf{M}_{1}^{\epsilon} & \mathbf{C}^{T} \mathbf{M}_{2}^{\mu^{-1}} \\
\mathbf{M}_{2}^{\mu^{-1}} \mathbf{C} & 0
\end{array}\right]\left[\begin{array}{l}
\dot{\vec{e}} \\
\vec{b}
\end{array}\right]=\left[\begin{array}{c}
0 \\
-\mathbf{M}_{2}^{\mu^{-1}} \ddot{\vec{b}}
\end{array}\right]
$$


Due to the zero entry on the right hand side (3.114) can be written in a form similar to (3.118). The second order equation for the magnetic flux density including the source terms is given in (3.115)

$$
\mathbf{M}_{2} \ddot{\vec{b}}+\mathbf{Y} \dot{\vec{b}}-\mathbf{Z} \vec{b}=-\mathbf{M}_{2} \mathbf{C}\left(\mathbf{M}_{1}^{\epsilon^{-1}}\right)^{-1} \mathbf{C}^{T} \mathbf{M}_{2} \vec{b}+\mathbf{G} \vec{j}-\mathbf{M}_{2} \dot{\vec{m}}+\mathbf{M}_{2}^{\left(\epsilon^{-1} \sigma_{M}\right)} \vec{m}
$$

where

$$
\begin{aligned}
\mathbf{Y}_{i, j} & =\left(\left(\sigma_{M}-\epsilon \sigma_{E}\right) \vec{F}_{i}, \vec{F}_{j}\right) \\
\mathbf{Z}_{i, j} & =\left(\left(\sigma_{M} \epsilon^{-1} \sigma_{E}\right) \vec{F}_{i}, \vec{F}_{j}\right) \\
\mathbf{G}_{i, j} & =\left(\epsilon^{-1} \nabla \times \vec{F}_{i}, \vec{F}_{j}\right)
\end{aligned}
$$

The variational form of the second order electric field wave equation (2.28) is shown in the variational form of this equation becomes (3.117).

$$
\begin{array}{r}
\frac{\partial^{2}}{\partial t^{2}}\left(\epsilon \vec{E}, \vec{E}^{*}\right)+\frac{\partial}{\partial t}\left(\left(\sigma_{E}+\mu^{-1} \sigma_{M} \epsilon\right) \vec{E}, \vec{E}^{*}\right)+\left(\mu^{-1} \sigma_{M} \sigma_{E} \vec{E}, \vec{E}^{*}\right)= \\
-\left(\mu^{-1} \nabla \times \vec{E}, \nabla \times \vec{E}^{*}\right)-\left(\mu^{-1} \sigma_{M} \vec{J}_{s}, \vec{E}^{*}\right)-\left(\mu^{-1} \nabla \times \vec{M}_{s}, \vec{E}^{*}\right) \\
-\frac{\partial}{\partial t}\left(\vec{J}_{s}, \vec{E}^{*}\right)-\oint_{\Gamma} \mu^{-1}\left(\vec{E}^{*} \times \nabla \times \vec{E}\right) \cdot \hat{n} \forall \vec{E}^{*}
\end{array}
$$

Entering the expansions for the electric field and electric and magnetic current densities results in the second order electric wave equation (3.118)

$$
\mathbf{M}_{1}^{\epsilon} \ddot{\vec{e}}+\mathbf{T} \dot{\vec{e}}+\mathbf{U} \vec{e}=-\mathbf{K}_{1}^{\mu^{-1}} \vec{e}-\mathbf{V} \vec{j}-\mathbf{C}^{T} \vec{m}-\mathbf{X} \dot{\vec{j}}-\oint_{\Gamma}\left(\mu^{-1} \vec{W}_{j} \times \nabla \times \vec{E}\right) \cdot \hat{n}
$$

where

$$
\begin{aligned}
& \mathbf{T}_{i, j}=\left(\left(\sigma_{E}+\mu^{-1} \sigma_{M} \epsilon\right) \vec{W}_{i}, \vec{W}_{j}\right) \\
& \mathbf{U}_{i, j}=\left(\left(\mu^{-1} \sigma_{M} \sigma_{E}\right) \vec{W}_{i}, \vec{W}_{j}\right) \\
& \mathbf{V}_{i, j}=\left(\vec{W}_{i}, \mu^{-1} \sigma_{M} \vec{F}_{j}\right) \\
& \mathbf{X}_{i, j}=\left(\vec{W}_{i}, \vec{F}_{j}\right)
\end{aligned}
$$


The essential and natural boundary conditions can be determined from the boundary condition term in the variational equation (3.117). In this wave equation the essential boundary condition corresponds to the Dirichlet boundary condition and the natural boundary condition corresponds to the natural boundary condition (3.119).

$$
\begin{array}{r}
\vec{E} \times \hat{n}=\vec{D} \text { on } \Gamma_{D} \\
\nabla \times \vec{E} \times \hat{n}=\vec{N} \text { on } \Gamma_{N}
\end{array}
$$

The boundary term can be evaluated in terms of the discrete basis functions and is shown in (3.120). The resulting matrix is zero due to the choice of test space.

$$
\oint_{\Gamma} \mu^{-1}\left(\vec{E}^{*} \times \nabla \times \vec{E}\right) \cdot \hat{n}=\left(\mu^{-1} \nabla \times \vec{W}_{i}, \vec{W}_{j} \times \hat{n}\right)_{\Gamma}=0, \vec{W}_{j} \in \mathcal{H}_{0}(\text { curl })
$$

Rewriting the discrete second order electric wave equation in a charge and source free region results in the mixed form of this wave equation (3.121).

$$
\left[\begin{array}{cc}
\mathbf{M}_{2}^{\mu^{-1}} & \mathbf{M}_{2}^{\mu^{-1}} \mathbf{C} \\
\mathbf{C}^{T} \mathbf{M}_{2}^{\mu^{-1}} & 0
\end{array}\right]\left[\begin{array}{c}
\dot{\vec{b}} \\
\vec{e}
\end{array}\right]=\left[\begin{array}{c}
0 \\
-\mathbf{M}_{1}^{\epsilon} \ddot{\vec{e}}
\end{array}\right]
$$

In this form it is apparent that the 1-form stiffness matrix $\mathbf{K}_{1}$ can be written in a alternative form. This form is shown in (3.122).

$$
\mathbf{K}_{1}^{\mu^{-1}}=\mathbf{C}^{T} \mathbf{M}_{2}^{\mu^{-1}} \mathbf{C}
$$

\subsubsection{Linear acoustic vector wave equations}

The variational form of the second order adjoint acoustic wave equation was shown above in (3.96). The spaces used to discretize the adjoint acoustic vector wave equation are the spaces used for the natural scalar wave equation. This leads to the 
discretizations for the velocity (3.89) and the pressure (3.84). For the vector wave equation the boundary conditions are imposed on the velocity instead of the pressure. In this case the boundary conditions are given by (3.123).

$$
\begin{gathered}
\vec{v} \times \hat{n}=\vec{D} \text { on } \Gamma_{D} \\
\vec{v} \cdot \hat{n}=\vec{N} \text { on } \Gamma_{N}
\end{gathered}
$$

The discrete equations are the same as (3.90) with the second derivative on the velocity instead of the pressure.

$$
\begin{array}{r}
\mathbf{M}_{0} \dot{\vec{\alpha}}=\mathbf{G}^{T} \mathbf{M}_{1} \vec{\beta} \\
\mathbf{M}_{1} \ddot{\vec{\beta}}=-c_{l}^{2} \mathbf{M}_{1} \mathbf{G} \dot{\vec{\alpha}}
\end{array}
$$

The boundary matrix in this case evaluates to zero (3.125) due to the choice of the test space used for $\vec{v}^{*}$ just as in the natural scalar wave equation case.

$$
\oint_{\Gamma} N_{j}(\vec{v} \cdot \hat{n})=\oint_{\Gamma} N_{j}\left(\vec{W}_{i} \cdot \hat{n}\right)=0, N_{j} \in H_{0}(\nabla, \bar{\Omega})
$$

The mixed form discretization of (3.126) with $c_{l}=1$ becomes:

$$
\left[\begin{array}{cc}
\mathbf{M}_{0} & -\mathbf{G}^{T} \mathbf{M}_{1} \\
-\mathbf{G} & 0
\end{array}\right]\left[\begin{array}{c}
\dot{\vec{\alpha}} \\
\vec{\beta}
\end{array}\right]=\left[\begin{array}{c}
0 \\
\ddot{\vec{\beta}}
\end{array}\right]
$$

In symmetrized form with (3.126) becomes:

$$
\left[\begin{array}{cc}
\mathbf{M}_{0} & -\mathbf{G}^{T} \mathbf{M}_{1} \\
-\mathbf{M}_{1} \mathbf{G} & 0
\end{array}\right]\left[\begin{array}{c}
\dot{\vec{\beta}} \\
\vec{\alpha}
\end{array}\right]=\left[\begin{array}{c}
0 \\
\mathbf{M}_{1} \ddot{\vec{\alpha}}
\end{array}\right]
$$

The variational form of the second order vector acoustic wave equation (2.31) is shown in (3.128).

$$
\frac{\partial^{2}}{\partial t^{2}}\left(\vec{v}, \vec{v}^{*}\right)=-\left(c_{l}^{2} \nabla \cdot \vec{v}, \nabla \cdot \vec{v}^{*}\right)+c_{l}^{2} \oint_{\Gamma}(\nabla \cdot \vec{v}) \vec{v}^{*} \cdot \hat{n} \forall \vec{v}^{*}
$$


From the boundary term of the variational form the boundary conditions can determined. The boundary conditions for this variational form are shown in (3.129).

$$
\begin{aligned}
& \vec{v} \cdot \hat{n}=\vec{g} \text { on } \Gamma_{D} \\
& \nabla \cdot \vec{v}=h \text { on } \Gamma_{N}
\end{aligned}
$$

The velocity will be discretized in terms of 2-form face basis functions.

$$
\vec{v}=\sum_{i=1}^{N_{\text {faces }}} \beta_{i} \vec{F}_{i}
$$

The discrete acoustic vector wave equation is shown in (3.131).

$$
\mathbf{M}_{2} \ddot{\vec{\beta}}=-\left(\mathbf{K}_{2}^{c_{l}^{2}}-\oint_{\Gamma}\left(\nabla \cdot \vec{F}_{j}\right) \vec{F}_{i}^{*} \cdot \hat{n}\right) \vec{\beta}
$$

where $\mathbf{K}_{2}^{c_{l}^{2}}$ is symmetric positive semi-definite 2 -form stiffness matrix with material equal to the sound speed squared $c_{l}^{2}$. The boundary term in the discrete acoustic vector wave equation evaluates to zero due to the choice of test space.

$$
\oint_{\Gamma}\left(\nabla \cdot \vec{F}_{j} \vec{F}_{i}^{*} \cdot \hat{n}\right)=\left(\nabla \cdot \vec{F}_{j}, \vec{F}_{i} \cdot \hat{n}\right)_{\Gamma}=0, \vec{F}_{i} \in \mathcal{H}_{0}(\text { div })
$$

In symmetrized mixed form this becomes:

$$
\left[\begin{array}{cc}
\mathbf{M}_{2} & -\mathbf{M}_{3} \mathbf{D} \\
-\mathbf{D}^{T} \mathbf{M}_{3} & 0
\end{array}\right]\left[\begin{array}{c}
\dot{\vec{\alpha}} \\
\vec{\beta}
\end{array}\right]=\left[\begin{array}{c}
0 \\
\mathbf{M}_{2} \ddot{\vec{\beta}}
\end{array}\right]
$$

Reducing the mixed form to a single equation shows the 2-form stiffness matrix can be written in terms of the divergence differential operator matrix $\mathbf{D}$ and the 3-form mass matrix $\mathbf{M}_{3}$ (3.134).

$$
\mathbf{K}_{2}=\mathbf{D}^{T} \mathbf{M}_{3} \mathbf{D}
$$




\subsubsection{Linear elastic wave equations}

The variational form of the second order displacement equation for a linear isotropic elastic medium is given by (3.135)

$$
\frac{\partial^{2}}{\partial t^{2}}\left(\vec{u}, \vec{u}^{*}\right)=-\left(\mu \nabla \times \nabla \times \vec{u}, \vec{u}^{*}\right)+\left((2 \mu+\lambda) \nabla\left(\nabla \cdot \vec{u}, \vec{u}^{*}\right)-\left(\vec{f}, \vec{u}^{*}\right) \forall \vec{u}^{*}\right.
$$

To form the discretization of this equation with $\vec{u}$ represented as a 2 -form (3.136), the natural form of the Grad-Div operator is combined with the adjoint form of the Curl-Curl giving discrete version of this equation with no body forces present (3.137)

$$
\begin{gathered}
\vec{u}=\sum_{i=1}^{N_{\text {faces }}} \alpha_{i} \vec{F}_{i} \\
\mathbf{M}_{2} \frac{\partial^{2} \vec{\alpha}}{\partial t^{2}}=-\left\{\mu \mathbf{M}_{2} \mathbf{C M}_{1}^{-1} \mathbf{C}^{T} \mathbf{M}_{2}+\mathbf{K}_{2}^{(2 \mu+\lambda)}\right\} \vec{\alpha}
\end{gathered}
$$

For $\vec{u}$ expanded as a 1-form (3.138) the natural form of the Curl-Curl operator is combined with the adjoint form of the Grad-Div operator giving (3.139)

$$
\begin{gathered}
\vec{u}=\sum_{i=1}^{N_{\text {edges }}} \beta_{i} \vec{W}_{i} \\
\mathbf{M}_{1} \frac{\partial^{2} \vec{\beta}}{\partial t^{2}}=-\left\{\mathbf{K}_{1}^{\mu}+(2 \mu+\lambda)\left(\mathbf{M}_{1} \mathbf{G M}_{0}^{-1} \mathbf{G}^{T} \mathbf{M}_{1}\right)\right\} \vec{\beta}
\end{gathered}
$$

\subsubsection{Linear magnetohydrodynamic wave equations}

The linear magnetohydrodynamics equations represent equations that are outside

of the discrete forms IBVP representations shown in Chapter 2. The linear magnetohydrodynamics equations (2.48) have the variational forms listed in (3.143), (3.145) and (3.147). Each of this variational forms will be analyzed in turn resulting in a mixed form discrete system. 
The 2-form velocity $\vec{v}=\vec{v}_{1}$ and magnetic flux density $\vec{B}=\vec{B}_{1}$ will be in the $\mathcal{H}($ div $)$ space, their corresponding test variables $\vec{v}^{*}$ and $\vec{B}^{*}$ are in the space $\mathcal{H}_{0}($ div $)$. The 3-form pressure $P_{1}=P$ and test variable $P^{*}$ will be in $L^{2}$. Both the perturbed velocity and magnetic field will be expanded in terms of 2 -form face basis functions $\vec{F}$, while the perturbed pressure will be expanded in terms of 3-form volume basis functions $S$.

$$
\begin{aligned}
& \vec{v}=\sum_{i=1}^{N_{\text {faces }}} \beta_{i} \vec{F}_{i} \\
& \vec{B}=\sum_{i=1}^{N_{\text {faces }}} b_{i} \vec{F}_{i} \\
& P=\sum_{i=1}^{N_{\text {vols }}} p_{i} S_{i}
\end{aligned}
$$

\section{Pressure equation}

$$
\frac{\partial}{\partial t}\left(P, P^{*}\right)=-\gamma P_{0}\left(\nabla \cdot \vec{v}, P^{*}\right) \forall P^{*} \in L^{2}
$$

No integration by parts is required to form this equation do to the inclusion $\nabla \cdot \vec{v} \in$ $L^{2}$. The resulting discrete equation is shown in (3.144).

$$
\dot{\vec{p}}=-\gamma P_{0} \mathbf{D} \vec{\beta}
$$

\section{Velocity equation}

The variational form for the first order velocity equation is shown in (3.145).

$$
\begin{array}{r}
\rho_{0} \frac{\partial}{\partial t}\left(\vec{v}, \vec{v}^{*}\right)=\left(P, \nabla \cdot \vec{v}^{*}\right)-\left(\frac{1}{\mu_{0}} \vec{B}_{0} \times \vec{v}^{*}, \vec{B}\right) \\
-\oint_{\Gamma} P \vec{v}^{*} \cdot \hat{n}+\oint_{\Gamma} \vec{B} \times\left(\frac{1}{\mu_{0}} \vec{B}_{0} \times \vec{v}^{*}\right) \cdot \hat{n} \forall \vec{v}^{*} \in \mathcal{H}_{0}(d i v)
\end{array}
$$


This variational form uses (B.2) and the combination of the operators (B.1) and (B.3) to derive the variational formulation. Due to the choice of test space $\mathcal{H}_{0}($ div $)$, both of the boundary terms are zero.

The discrete form of this equation (3.146) is formed from the normal pressure gradient term already derived above and the new cross term which will be examined below.

$$
\mathbf{M}_{2} \dot{\vec{\beta}}=c_{l}^{2} \mathbf{D}^{T} \mathbf{M}_{3} \vec{p}-\mathbf{M}_{\times}^{T}\left(\frac{\vec{v}_{a}}{\sqrt{\mu_{0} \rho_{0}}}\right) \mathbf{M}_{1}^{-1} \mathbf{C}^{T} \mathbf{M}_{2} \vec{b}
$$

\section{Magnetic Field Part}

The second part on the right hand side of the velocity equation over the entire domain is given by:

$$
-\int \frac{\vec{B}_{0}}{\mu_{0}} \times \nabla \times \vec{B}_{1} \cdot \vec{F}_{j} d \Omega
$$

Using the vector identity:

$$
\vec{A} \cdot(\vec{B} \times \vec{C})=\vec{B} \cdot(\vec{C} \times \vec{A})=\vec{C} \cdot(\vec{A} \times \vec{B})
$$

gives:

$$
-\int \nabla \times \vec{B}_{1} \cdot\left(\vec{F}_{j} \times \frac{\vec{B}_{0}}{\mu_{0}}\right) d \Omega
$$

swapping the cross product terms:

$$
\int \nabla \times \vec{B}_{1} \cdot\left(\frac{\vec{B}_{0}}{\mu_{0}} \times \vec{F}_{j}\right) d \Omega
$$

Since the quantity $\vec{B}_{0}$ is constant throughout each cell the quantity $\frac{\vec{B}_{0}}{\mu_{0}} \times \vec{F}_{j} \in \mathcal{W}$ if $\vec{h}=\frac{\vec{B}_{0}}{\mu_{0}} \times \vec{F}_{j} \in \mathcal{W}$, then:

$$
\int \nabla \times \vec{B}_{1} \cdot \vec{h} d \Omega
$$


using the identity:

$$
\nabla \cdot(\vec{a} \times \vec{b})=\vec{b} \cdot(\nabla \times a)-\vec{a} \cdot(\nabla \times b)
$$

integration by parts gives:

$$
\int \nabla \times \vec{B}_{1} \cdot \vec{h} d \Omega=\int \nabla \cdot\left(\vec{B}_{1} \times \vec{h}\right) d \Omega+\int \nabla \times \vec{h} \cdot \vec{B}_{1} d \Omega
$$

Integrating the first part on the right hand side:

$$
\int \nabla \cdot\left(\vec{B}_{1} \times \vec{h}\right) d \Omega=\int \vec{B}_{1} \times \vec{h} \cdot \hat{n} d \Gamma
$$

this equation is zero if $\vec{B} \cdot \vec{n}=0$ on $\Gamma$. Using the inclusion relation $\nabla \times \vec{W}_{i} \in \mathcal{F}$ the second term on the right hand side becomes in discrete form:

$$
\mathbf{C}^{T} \mathbf{M}_{2} \vec{b}
$$

This is without the cross product for $\vec{h}$,including the definition. This term becomes:

$$
\left(-\mathbf{M}_{\times}^{T}\right)\left(\frac{\vec{v}_{a}}{\sqrt{\mu_{0} \rho_{0}}}\right) \mathbf{M}_{1}^{-1} \mathbf{C}^{T} \mathbf{M}_{2} \vec{b}+\int\left(\frac{\vec{B}_{0}}{\mu_{0}} \times \vec{F}_{j}\right) \cdot\left(\hat{n} \times \vec{B}_{1}\right) d \Gamma
$$

where $\vec{v}_{a}=\frac{\vec{B}_{0}}{\sqrt{\mu_{0} \rho_{0}}}$ and the entire velocity equation was given in (3.146). The $M_{\times}$ matrix will be discussed below.

\section{Magnetic Field Equation}

$$
\frac{\partial}{\partial t}\left(\vec{B}, \vec{B}^{*}\right)=\left(\nabla \times\left(\vec{v} \times \vec{B}_{0}\right), \vec{B}^{*}\right) \forall \vec{B}^{*}
$$

The right hand side is similar to the magnetic field term in the velocity equation. Setting $\vec{g}=\sqrt{\mu_{0} \rho_{0}} \vec{v}_{a} \times \vec{v}_{1}$ and integrating over the entire domain:

$$
-\int \nabla \times(\vec{g}) \cdot \vec{F}_{j} d \Omega
$$


Using the inclusion relation: $\nabla \times \vec{W}_{i} \in \mathcal{F}$ the right hand side becomes:

$$
-\mathbf{M}_{2} \mathbf{C M}_{2}^{-1} \mathbf{M}_{\times}\left(\sqrt{\mu_{0} \rho_{0}} \vec{v}_{a}\right) \vec{v}
$$

Combining these equations:

$$
\mathbf{M}_{2} \dot{\vec{b}}=-\mathbf{M}_{2} \mathbf{C M}_{1}^{-1} \mathbf{M}_{\times}\left(\sqrt{\mu_{0} \rho_{0}} \vec{v}_{a}\right) \vec{v}
$$

This discrete formulation for the first order magnetic flux density equation becomes (3.148).

$$
\dot{\vec{b}}=-\mathbf{C M}_{1}^{-1} \mathbf{M}_{\times}\left(\sqrt{\mu_{0} \rho_{0}} \vec{v}_{a}\right) \vec{v}
$$

\section{Cross Product Matrix}

Given a quantity expanded in terms of the 2-form face basis functions such as the velocity (3.149).

$$
\vec{v}_{1}=\sum_{i=1}^{N_{\text {faces }}} \beta_{i} \vec{F}_{i}
$$

As shown in Chapter 2 the wedge product with a constant vector, which can also be expanded as a 2-form, results in a 1-form (3.150).

$$
\vec{F}_{i} \times \vec{B}_{0} \in \mathcal{W}
$$

The resulting quantity will be a 1-form expanded in the edge basis functions. This quantity is the electric field (3.151) derived form the infinite conductivity form of the Lorentz equation (3.152).

$$
\begin{gathered}
\vec{E}_{1}=\sum_{i=1}^{N_{\text {edges }}} e_{i} \vec{W}_{i} \\
\vec{E}=\vec{v} \times \vec{B}_{0}
\end{gathered}
$$


Performing the necessary inner products

$$
\sum_{i=1}^{N_{\text {edges }}}\left(\int \vec{W}_{i} \cdot \vec{W}_{j} d \Omega_{e}\right) e_{i}=\sum_{k=1}^{N_{\text {faces }}}\left(\int \vec{F}_{k} \cdot \vec{W}_{j} \times \vec{B}_{0} d \Omega_{e}\right) \beta_{i}
$$

Rearranging:

$$
\sum_{i=1}^{N_{\text {edges }}}\left(\int \vec{W}_{i} \cdot \vec{W}_{j} d \Omega_{e}\right) e_{i}=\sum_{k=1}^{N_{\text {faces }}}\left(\int \vec{B}_{0} \cdot \vec{W}_{j} \times \vec{F}_{k} d \Omega_{e}\right) \beta_{i}
$$

results in the matrix form of the 2 -form wedge product giving the cross product in vector calculus (3.153).

$$
\mathbf{M}_{e} \vec{e}=\mathbf{M}_{\times}\left(\vec{B}_{0}\right) \vec{\beta}
$$

where $\mathbf{M}_{\times}\left(\vec{B}_{0}\right)$ is given in (3.154).

$$
\mathbf{M}_{\times}\left(\vec{B}_{0}\right) \equiv \int \vec{B}_{0} \cdot \vec{W}_{j} \times \vec{F}_{k} d \Omega
$$

\subsubsection{Second order linear magnetohydrodynamics}

Taking the time derivative of the discrete velocity equation (3.155)

$$
\mathbf{M}_{f} \ddot{\vec{v}}=c_{l}^{2} \mathbf{D}^{T} \mathbf{M}_{3} \dot{\vec{\rho}}-\mathbf{M}_{\times}\left(\frac{\vec{v}_{a}}{\sqrt{\mu_{0} \rho_{0}}}\right) \mathbf{M}_{1}^{-1} \mathbf{C}^{T} \mathbf{M}_{2} \dot{\vec{b}}
$$

Substituting the discrete pressure (3.144) and magnetic field equations (3.148) gives the second order magnetohydrodynamics equation for velocity (3.156).

$$
\mathbf{M}_{2} \ddot{\vec{v}}=-c_{l}^{2} \mathbf{K}_{2} \vec{v}+\mathbf{M}_{\times}^{T}\left(\vec{v}_{a}\right) \mathbf{M}_{1}^{-1} \mathbf{K}_{1} \mathbf{M}_{1}^{-1} \mathbf{M}_{\times}\left(\vec{v}_{a}\right) \vec{v}
$$

To perform the wedge product for the 2-forms, they must first be converted to 1-forms using the Hodge star operator. These two operations can be seen in (3.156) where the 1-form mass matrices are inverted and coupled with the 1-from stiffness 
matrix $\mathbf{K}_{1}$ forming a discrete Hodge star operator. Once wedged the resulting 2form is wedged again to convert back to 1 -form indicated by the 2-form mass matrix inversion. 


\section{Chapter 4}

\section{Linear Algebraic System Analysis}

\subsection{Iterative Methods}

Disregarding the time derivative of the wave equations gives a linear algebraic system of equations (4.1)

$$
\mathbf{A} \vec{x}=\vec{b}
$$

where $\mathbf{A}$ in the case of the wave equations is a symmetric positive definite mass matrix,

$\vec{b}$ is formed from the stiffness matrix and any sources and $\vec{x}$ is the vector of degrees of freedom. Even though the time stepping algorithm is explicit, the finite element method requires a solution of this system at every time step. This requirement in turn constrains the solution method used for the system. An inefficient solver will make large problems intractable. For this reason iterative methods are used to solve the system in (4.1).

Two main types of algorithms are used to solve a system such as (4.1), direct(noniterative) and iterative methods. The direct methods entail factoring the matrix $\mathbf{A}$ 
using an algorithm such as Gaussian Elimination to obtain an upper triangular form. Once the matrix is factored, the last equation is trivial to solve and the solution can be used to solve the next to last equation and so on. This is refereed to as back substitution. An operation count for Gaussian elimination and back substitution for a matrix with $n$ rows and $n$ columns gives a result that is proportional to $n^{3}, \mathcal{O}\left(n^{3}\right)$. For one of the simulations in this dissertation the number of unknowns is on the order of $n=4 e 6$. Using 32 processors with a theoretical floating point operations per second count of 4 GFlops at maximum efficiency will take 15.8 years to factor the matrix making direct methods useless for large problems. Sparse direct methods such as SuperLU [44] can efficiently solve problems with a large number of degrees of freedom. In the case of this dissertation the linear system solution involves a wellconditioned mass matrix for which iterative methods are more efficient than sparse direct methods.

Iterative methods for (4.1) have much lower operation counts for very large problems. A good reference on iterative methods is [45]. The simplest iterative method is given in (4.2) where $l=0,1,2, \ldots$ and $\tau$ is a parameter.

$$
\vec{x}^{l+1}=\vec{x}^{l}-\tau\left(\mathbf{A} \vec{x}^{l}-\vec{b}\right)
$$

This method starts with an initial guess for $\vec{x}^{0}$ and successively applies the formula utilizing only matrix-vector multiplies.

Iterative methods are further classified into stationary and non-stationary methods. The stationary methods are comprised of the Jacobi,Gauss-Seidel and Symmetric Over Relaxation (SOR) iterative methods. These methods are best described by 
introducing a matrix splitting (4.3).

$$
\mathbf{A}=\mathbf{M}-\mathbf{N}
$$

This splitting is comprised of a matrix easy to invert $\mathbf{M}$ and the remainder $\mathbf{N}$. The iterative solution method for (4.1) using this splitting is listed in (4.4)

$$
\begin{array}{r}
\mathbf{M} \vec{x}^{k+1}=\mathbf{N} \vec{x}^{k}+\vec{b} \\
\vec{x}^{k+1}=\mathbf{M}^{-1} \mathbf{N} \vec{x}^{k}+\mathbf{M}^{-1} \vec{b}
\end{array}
$$

The three different types of methods can be classified using another matrix splitting (4.5). In this splitting the matrix $\mathbf{D}$ is the diagonal of $\mathbf{A},-\mathbf{E}$ is the lower triangular portion without the diagonal and $\mathbf{- F}$ is the upper triangular portion without the diagonal.

$$
\mathbf{A}=\mathbf{D}-\mathbf{E}-\mathbf{F}
$$

The Jacobi iterative method uses the matrices defined in (4.6) in the stationary iterative method (4.4). The $\mathbf{M}$ matrix is the diagonal which is trivial to invert and $\mathbf{N}$ is the remainder of the matrix $\mathbf{A}$.

$$
\begin{array}{r}
\mathbf{M}=\mathbf{D} \\
\mathbf{N}=\mathbf{E}+\mathbf{F}
\end{array}
$$

The Gauss-Seidel method incorporates more of the matrix $\mathbf{A}$ by including the entire lower triangular portion in $\mathbf{M}$ shown in (4.7).

$$
\begin{array}{r}
\mathbf{M}=\mathbf{D}-\mathbf{E} \\
\\
\mathbf{N}=\mathbf{F}
\end{array}
$$


The SOR method is a generalization of the Gauss-Seidel method. The splitting matrices contain arbitrary combinations of $\mathbf{D}$ parameterized by a scalar $0 \leq \omega \leq 2$ shown in (4.8).

$$
\begin{array}{r}
\mathbf{M}=\frac{1}{\omega} \mathbf{D}-\mathbf{E} \\
\mathbf{N}=\frac{1-\omega}{\omega} \mathbf{D}+\mathbf{F}
\end{array}
$$

For a value of $\omega=1$ the SOR method reduces to the Gauss-Seidel method. Determining the optimum value for SOR is difficult unless the spectrum of the matrix is known. Determining the optimum value is therefore accomplished by trial and error.

It can be shown that for the symmetric positive definite mass matrix solutions in this dissertation all of these methods will converge. The convergence requirement is given by $\rho\left(\mathbf{M}^{-1} \mathbf{N}\right)<1$ for Gauss-Seidel and SOR where $\rho\left(\mathbf{M}^{-1} \mathbf{N}\right)$ is the largest eigenvalue of the matrix $\mathbf{M}^{-1} \mathbf{N}$ and $\rho\left(\mathbf{D}^{-1} \mathbf{A}\right)<2$ for the Jacobi iterative method.

Non-stationary methods are methods with non-constant coefficients for the iteration and cannot be written in the form (4.4). These non-stationary iterative methods include the steepest descent method and the conjugate gradient method. Both of these methods use the minimization of the functional (4.9) to form the iteration $(4.10)$

$$
\begin{gathered}
f(\vec{x})=\frac{1}{2} \vec{x}^{T} \mathbf{A} \vec{x}-\vec{b}^{T} \vec{x} \\
x_{i}^{k+1}=x_{i}^{k}+\alpha^{k} d_{i}^{k} ; i=1, \ldots, n ; k=0,1,2, \ldots
\end{gathered}
$$

Here the values for $\alpha^{k}$ and $d_{i}^{k}$ represent the step length and search direction respectively. The algorithm determines the minimum by creating a gradient and following this gradient in the opposite direction until convergence. Because the gradient will 
not necessarily point to the global minimum a step length is used to limit the variable change. The difference between steepest descent and conjugate gradient involves the choices for $\alpha$ and $d$. It can be shown that the convergence of conjugate gradient is as good as optimum SOR without requiring knowledge of the spectrum, it can also be used on matrices with arbitrary sparsity patterns and can be easily preconditioned for faster convergence.

Below in Section 4.3 an analysis of the preconditioned conjugate gradient solution is documented. The preconditioning process further improves the convergence of the conjugate gradient method by improving the condition number of the matrix. The condition number is the ratio of the largest to smallest eigenvalues of the matrix. Convergence is a function of condition number; when the condition number can be made smaller, the convergence is increased.

\subsection{Differential Operator Stencils}

An informative method for analyzing the differential operators is to examine the connectivity for each operator on a uniform Cartesian mesh. Through this analysis it will be shown that the discrete differential forms method reduces to simple standard finite difference operators on Cartesian grids. All of the analysis performed on this operators is therefore valid for the discrete differential forms method as well.

The stencil of an operator describes the discrete connectivity and weighting coefficients for that operator. An example of a stencil is constructed for a two-dimensional

scalar Laplacian $\frac{\partial^{2} \phi}{\partial x^{2}}+\frac{\partial^{2} \phi}{\partial x^{2}}=0$ acting on a scalar function $\phi$. Using the central difference operator (4.11) for both partial differential terms results in the two-dimensional 
finite difference Laplacian (4.12) where $\Delta h=1$.

$$
\begin{gathered}
\frac{\partial^{2} \phi}{\partial x^{2}}=\frac{\phi^{i+1}-2 \phi^{i}+\phi^{i-1}}{\Delta h^{2}}+\mathcal{O}\left(\Delta h^{2}\right) \\
-\phi^{i+1, j+1}-\phi^{i+1, j-1}+4 \phi^{i, j}-\phi^{i-1, j+1}-\phi^{i-1, j-1}=0
\end{gathered}
$$

The values $\phi^{i, j}$ represent the value of the scalar function $\phi$ at a node described by the index $i$ in the x-direction and index $j$ in the $\mathrm{y}$ direction. The stencil corresponding to the scalar Laplacian (4.12) is shown in Figure 4.1.

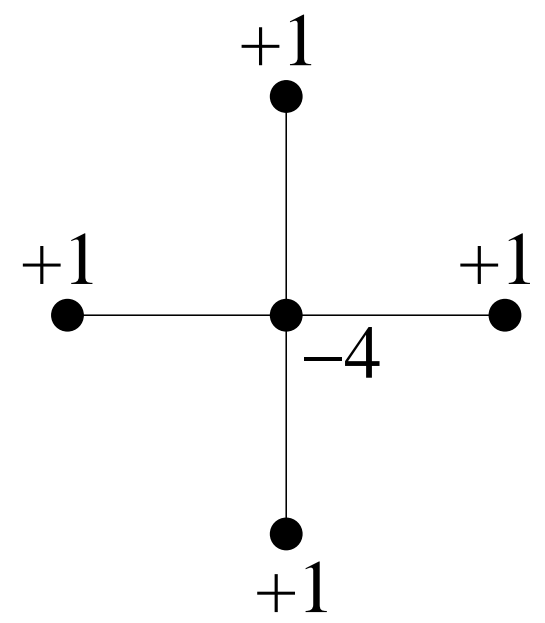

Figure 4.1: Scalar 2-Dimensional Laplacian

The mesh consists of a $3 x 3 x 3$ hexahedral domain with constant node spacing $h=1$. Each of the $p$-forms natural and adjoint operators are examined in their full form and mass lumped form if appropriate. The mass lumped form [46] of the operator involves a special integration rule that results in diagonal mass matrices. This would correspond to a mechanics problem where the mass of the system is not distributed continuously through the domain, but defined only at the nodes, thus lumping the mass at the nodes. Mass lumping is used in this section only to compute the stencils and is not advised for non-Cartesian grids. 


\subsubsection{Discrete Differential Operators}

The stencils for the discrete gradient $\mathbf{G}$, curl $\mathbf{C}$ and divergence operators $\mathbf{D}$ described in (3.63) are shown in Figure 4.2, Figure 4.3 and Figure 4.4 respectively.

The discrete gradient stencil shows the inclusion relation for the node in the center and the edges radiating out of the node. The coefficients are based on the arbitrary direction of each edge.

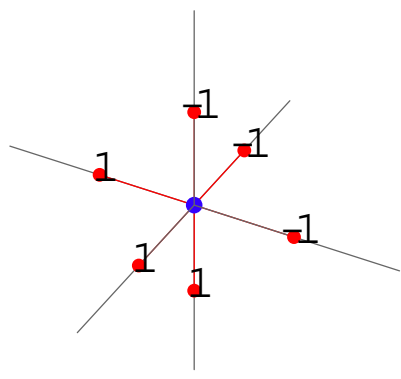

Figure 4.2: Discrete gradient operator

The discrete curl is the graphical representation of the inclusion relation relating the $\mathcal{W}^{h}$ to $\mathcal{F}^{h}$. The edge in the center is related to the four faces sharing that edge. The coefficients are based on the arbitrary edge and face directions.

The discrete divergence is the graphical representation of the inclusion relation relating the $\mathcal{F}^{h}$ to $L^{2}$. The face in the center shares only the two elements. The coefficients are determined by the connectivity of the face and the arbitrary direction of the face. 


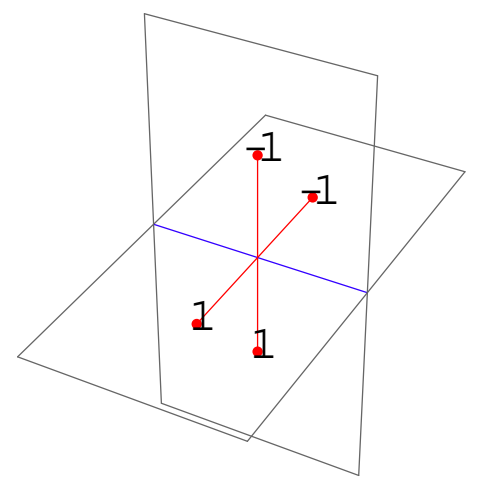

Figure 4.3: Discrete curl operator

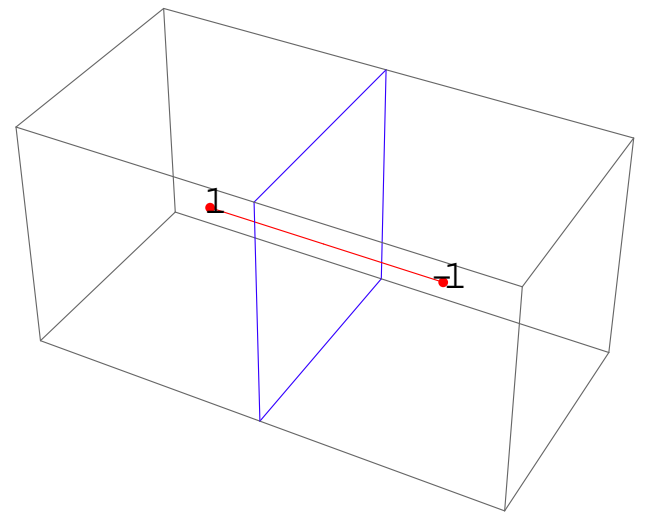

Figure 4.4: Discrete divergence operator 


\subsubsection{0-form operators}

In Figure 4.5 the stencil for the 0-form Div-Grad operator $\mathbf{M}_{0}^{-1} \mathbf{K}_{0}$ with lumped mass matrix $\mathbf{M}_{0}$ and full stiffness matrix $\mathbf{K}_{0}$ is presented. The matrices are constructed with unit materials. The figure shows the connectivity (lines) and matrix coefficient for a node in the center of the mesh forming a matrix stencil. This stencil corresponds to a 27 point finite difference stencil for a three-dimensional Laplacian.

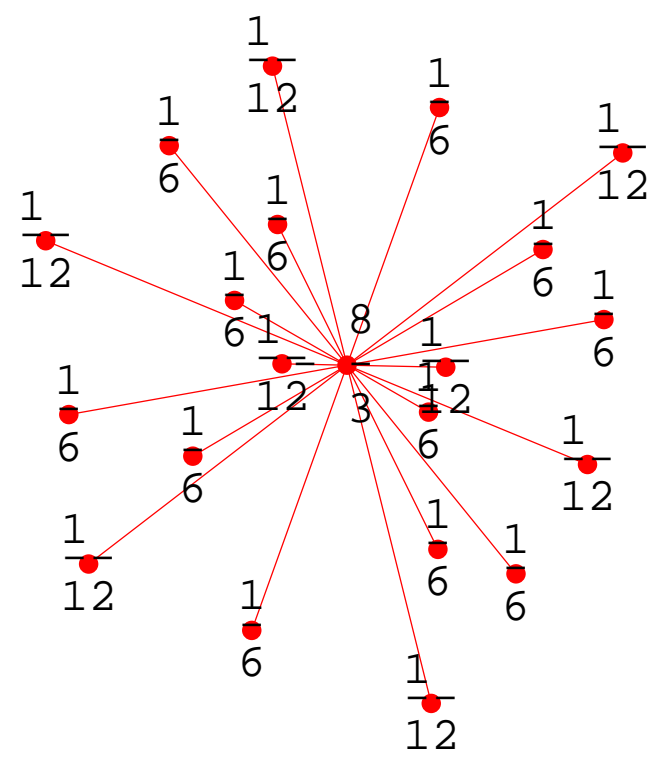

Figure 4.5: 0-form DivGrad operator

The accuracy of the stencil can be verified utilizing the tools used in finite difference analysis. A three dimensional scalar function is expanded in a Taylor series of several orders larger than the order of the differential operator. A sum of the product of the coefficients and the Taylor series evaluated at the coefficient's node produces a new series. This series will have only the lowest order non-zero coefficients of the series remaining. The lowest order coefficients determine the accuracy of the method. 
For the 27-point stencil listed above the accuracy is given in (4.13).

$$
h^{2}\left\{\frac{1}{12}\left(f_{400}+f_{040}+f_{004}\right)+\frac{1}{3}\left(f_{022}+f_{202}+f_{220}\right)\right\}+\text { H.O.T. }
$$

The notation used in for the coefficients in this equation show the order of the series coefficient $f_{i, j, k}$ where $i, j, k$ are the order of the coefficient for the $x, y$ and $z$ series terms respectively. In this case the stencil is second order accurate with all coefficients of lower order equal to zero. The term H.O.T. refers to the higher order terms in the expansion.

If both the mass matrix and stiffness matrices are mass lumped the common three-dimensional 7-point stencil is obtained and is shown in Figure 4.6.

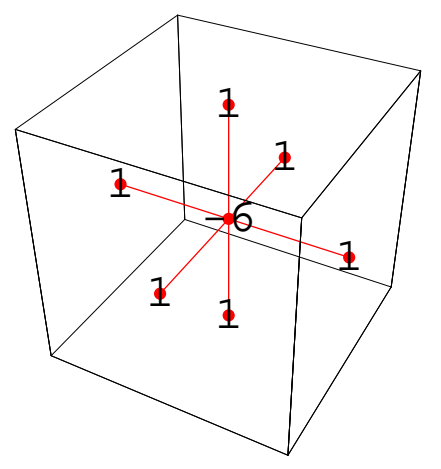

Figure 4.6: 0-form DivGrad Operator with Lumped Stiffness Matrix

Using the same series expansion for the three dimensional scalar function as in the non-lumped case, the accuracy of the operator is shown to be second order (4.14).

$$
\frac{h^{2}}{12}\left(f_{400}+f_{040}+f_{004}\right)+\text { H.O.T. }
$$

The discrete differential forms method on a uniform Cartesian with mass-lumped matrices reduces to the commonly used finite difference stencils for the 0-form differential operators. 


\subsubsection{1-form operators}

The three components of the 1-form natural Curl-Curl operator with lumped mass matrix and unit materials, $\mathbf{M}_{1}^{-1} \mathbf{K}_{1}$, are shown in Figure 4.7. The numbers represent the non-zero coefficients of the system located for a specific edge in the mesh located at the midpoints of each corresponding edge. The finite difference operator for the Curl-
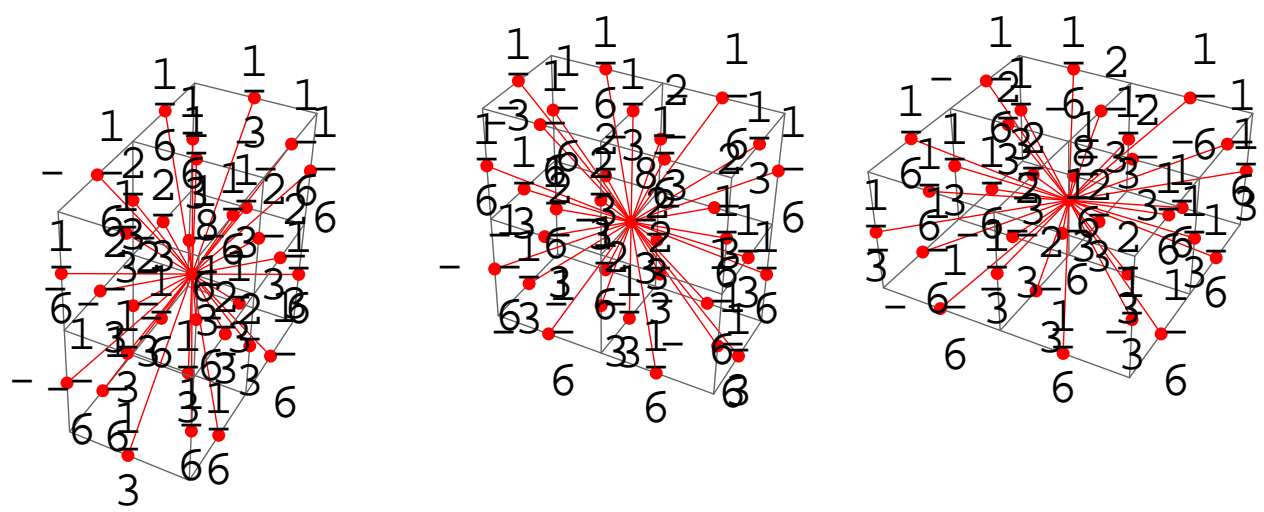

Figure 4.7: 1-form Curl-Curl Operators

Curl operator involves three separate operators for the resulting three directions of the operator. The accuracy of the above lumped finite element operator can be analyzed by taking each of the three operator stencils shown in Figure 4.7 and expanding them in Taylor series. The sum of the individual component's Taylor series results in a compound series. The series includes the operator and the truncation error of the equivalent finite difference stencil plus higher order terms. The finite difference stencil for the Curl-Curl operator is second order accurate. The sum of the Taylor series for the lumped Curl-Curl operator is shown in (4.15).

$$
\begin{array}{r}
h^{2}\left\{\frac{1}{3}\left(f_{400}+f_{004}+f_{040}\right)+\frac{1}{6}\left(f_{220}+f_{202}+f_{022}-f_{112}-f_{121}-f_{211}\right)\right. \\
-\frac{1}{12}\left(f_{130}+f_{310}+f_{103}+f_{301}+f_{013}+f_{031}\right\}+\text { H.O.T. }
\end{array}
$$


The lowest order term that is not part of the operator is proportional to $h^{2}$ on an orthogonal structured mesh with constant mesh spacing giving a second order accurate operator.

If both the mass matrix and the stiffness matrix are lumped the Curl-Curl operator reduces to the simplest finite difference stencil shown in Figure 4.8. Although the
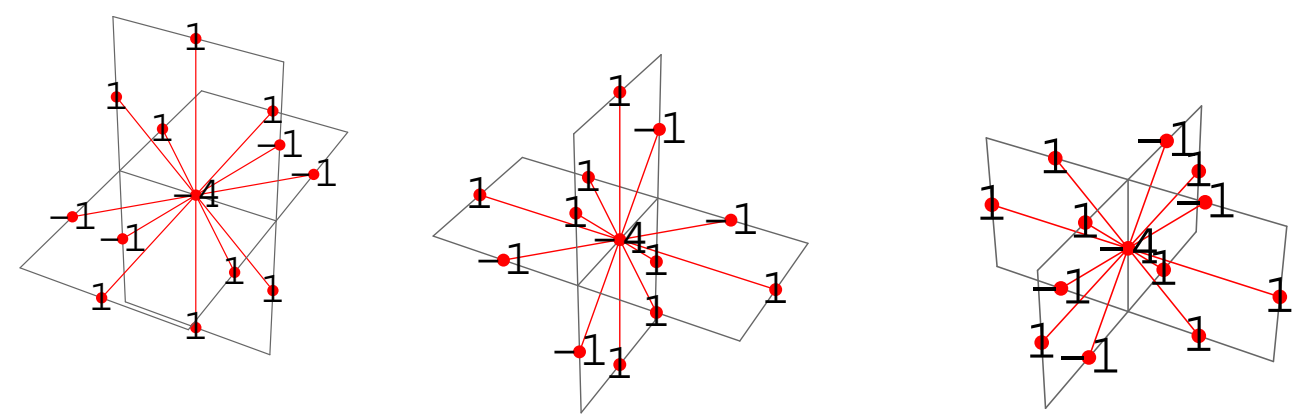

Figure 4.8: 1-form Curl-Curl Operator with Lumped Stiffness Matrix

truncation error is also second order accurate the truncation error (4.16) for the lumped mass matrix and lumped stiffness matrix is much simpler due to the reduced connectivity of the stencil.

$$
\frac{h^{2}}{12}\left(f_{400}+f_{004}+f_{040}-f_{130}-f_{310}-f_{103}-f_{301}-f_{013}-f_{031}\right)+\text { H.O.T. }
$$

The three components of the 1-form adjoint Grad-Div operator $\mathbf{G M}_{0}^{-1} \mathbf{G}^{T} \mathbf{M}_{1}$ with lumped mass matrices and unit materials are shown in Figure 4.9. This stencil corresponds to a standard second order Grad-Div finite difference stencil. The stencil for the x-component of the operator $\frac{\partial^{2}}{\partial x^{2}}+\frac{\partial}{\partial x} \frac{\partial}{\partial y}+\frac{\partial}{\partial x} \frac{\partial}{\partial z}$ is expanded in a central difference operator for the second order x-derivative while the cross terms are expanded 

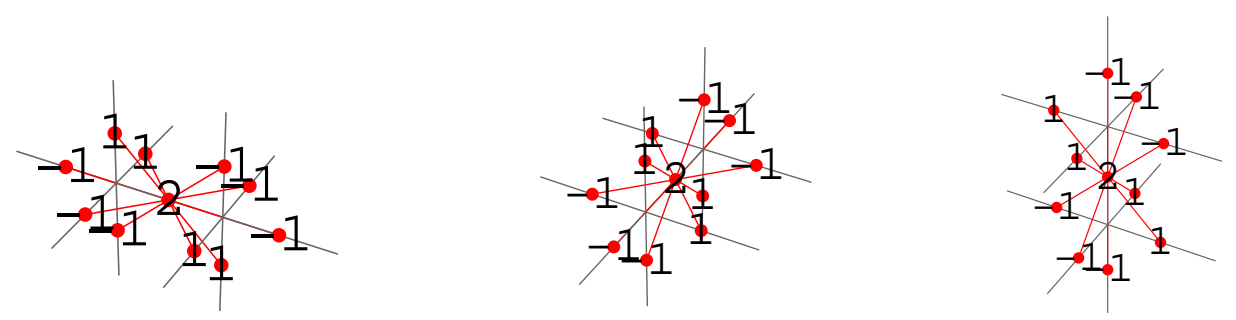

Figure 4.9: 1-form Adjoint Grad-Div Operator

in central difference at half intervals. The truncation error for this operator (4.17) is the same as the finite difference operator and is second order accurate.

$$
\frac{h^{2}}{12}\left(f_{400}+f_{004}+f_{040}-f_{130}-f_{310}-f_{103}-f_{301}-f_{013}-f_{031}\right)+\text { H.O.T. }
$$

Using the vector identity (B.6) the Curl-Curl and Grad-Div operators can be combined into the 1 -form vector Laplacian. As can be seen in the Figure 4.10 the three components of the stencils are decoupled. They do not share any degrees of freedom as in the three components of the Curl-Curl and Grad-Div operators. Each component of the vector Laplacian is the same as a three-dimensional scalar Laplacian stencil.

The three decoupled operators in the 1-form vector Laplacian result in a simplified truncation error (4.18) when combined and expanded in the Curl-Curl and Grad-Div operators.

$$
\frac{h^{2}}{12}\left(f_{400}+f_{004}+f_{040}\right)+\text { H.O.T. }
$$



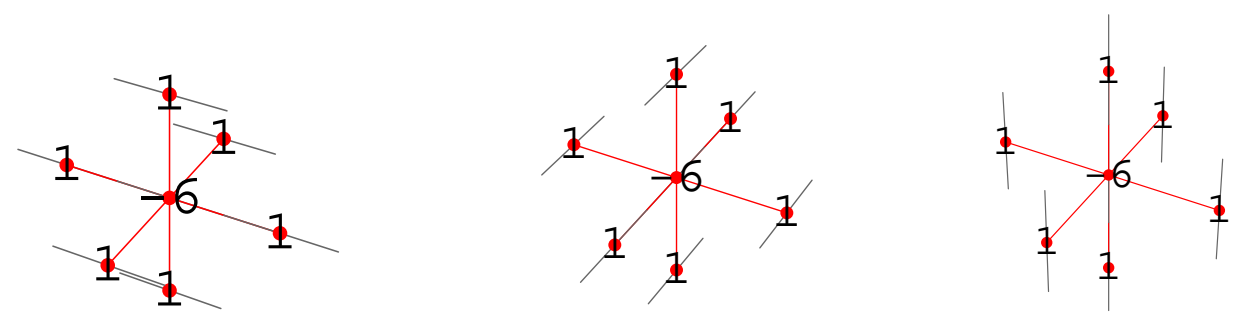

Figure 4.10: 1-form Laplacian

\subsubsection{2-form operators}

The 2-form operators from the natural Grad-Div, adjoint Curl-Curl and Vector Laplacian can all be examined as in the Section 4.2.3. Since the operators are defined for the 2-form face basis functions the stencils use the face centroids as the stencil locations instead of the edge midpoints as in the 1-from case.

The natural Grad-Div operator is shown in Figure 4.11. This stencil corresponds o the operator $\mathbf{M}_{2}^{-1} \mathbf{K}_{2}$ with a mass-lumped mass matrix and unit materials. If both the mass matrix and the stiffness matrix are lumped in this case the resulting stencil is the same.

If the 2-form Grad-Div operator is examined for accuracy as in the section for 1-form operators, the resulting truncation error (4.19) is second order accurate and is the same as the 1-form Grad-Div truncation error.

$$
\frac{h^{2}}{12}\left(f_{400}+f_{004}+f_{040}-f_{130}-f_{310}-f_{103}-f_{301}-f_{013}-f_{031}\right)+\text { H.O.T. }
$$

The three components of the vector adjoint Curl-Curl operator for the 2-forms $\mathbf{C M}_{1}^{-1} \mathbf{C}^{T} \mathbf{M}_{2}$ are shown in Figure 4.12. This stencil shows the same configuration 

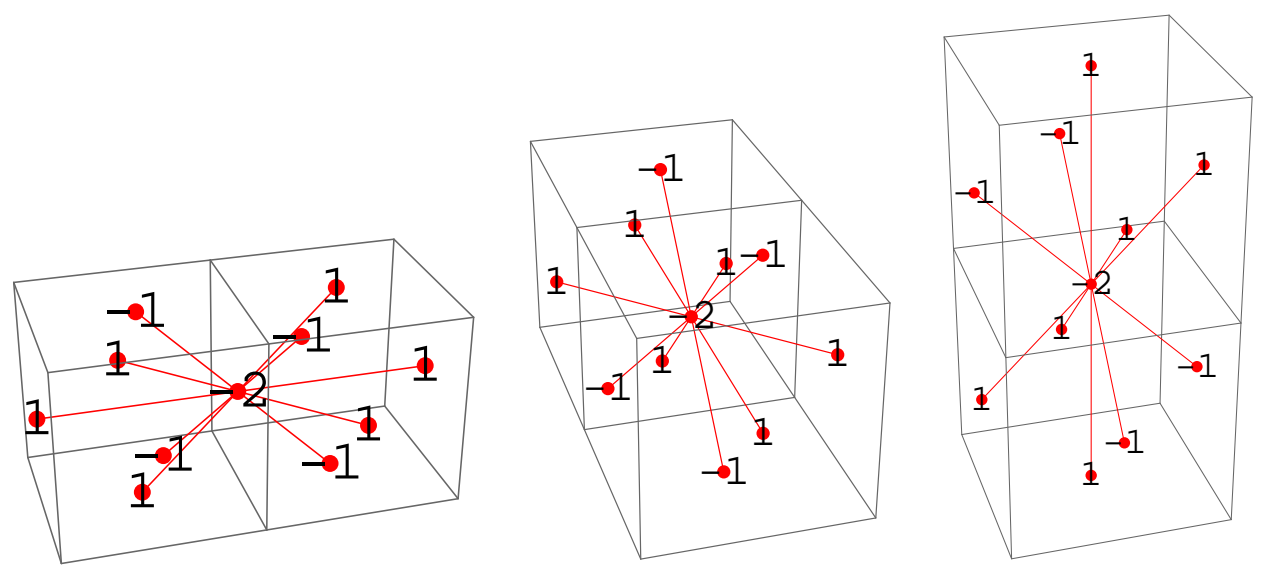

Figure 4.11: 2-form Grad-Div Operator
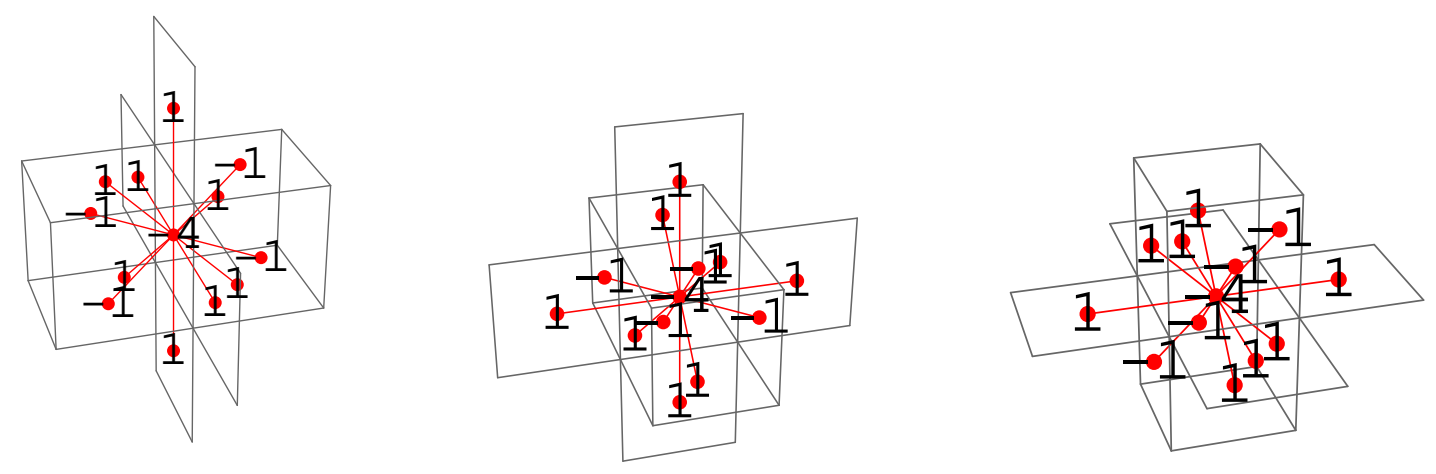

Figure 4.12: 2-form Adjoint Curl-Curl Operator 
as in the 1-form Curl-Curl operator, Figure 4.8, with both mass and stiffness matrix lumped. The difference is only in the location of the coefficients. The truncation error for the 2-form Curl-Curl operator (4.20) is the same as in the 1-form Curl-Curl operator .

$$
\frac{h^{2}}{12}\left(f_{400}+f_{004}+f_{040}-f_{130}-f_{310}-f_{103}-f_{301}-f_{013}-f_{031}\right)+\text { H.O.T. }
$$

The 2-form Grad-Div and adjoint Curl-Curl operator can be combined to form the vector Laplacian as in the section for 1-form operators. The three components of the operator are shown in Figure 4.13. Just as in the 1-form vector Laplacian, the three stencils for the 2-form vector Laplacian are decoupled and the stencil for each component is the same as a three-dimensional scalar Laplacian.
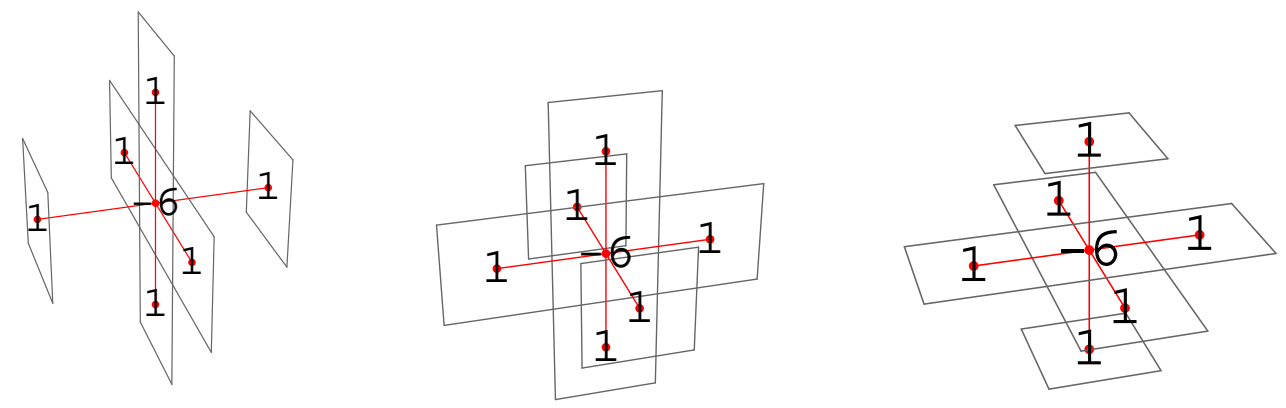

Figure 4.13: 2-form Laplacian

The truncation error for the vector Laplacian (4.21) is identical to the 1-form Laplacian.

$$
\frac{h^{2}}{12}\left(f_{400}+f_{004}+f_{040}\right)+\text { H.O.T. }
$$




\subsubsection{3-form operators}

The adjoint DivGrad operator $\mathbf{D} \mathbf{M}_{2}^{-1} \mathbf{D}^{T} \mathbf{M}_{3}$ developed first by Raviart and Thomas in two dimensions is shown in mass lumped form for the three dimensional operator in Figure 4.14. This operator corresponds to the common 7-point stencil using the cell centers instead of the node centering as in the 0-form operator.

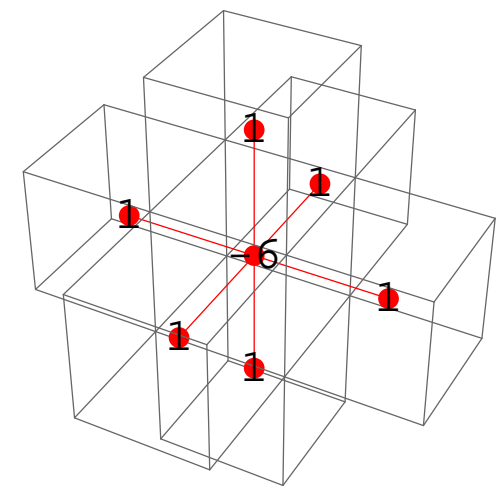

Figure 4.14: 3-form DivGrad operator

The Taylor expansion for the stencil given in Figure 4.14 is shown in (4.22).

$$
\frac{h^{2}}{12}\left(f_{400}+f_{040}+f_{040}\right)+\text { H.O.T. }
$$

Just as in the 0 -form scalar Laplacian case this operator is second order accurate.

\subsection{Mass Matrix Solution Scaling}

The discrete wave equations derived in the previous chapter include a linear solve of the mass matrix for the explicit time stepping method. Lower order integration 
rules or mass lumping can be used to diagonalize the matrix but in this dissertation the non-lumped consistent mass matrices are used. The efficient solution of the system is therefore critical. In this section the scaling properties of the mass matrix will be analyzed, this research is presented in detail in [47].

The domain is a two-dimensional square, $[0,1] x[0,1]$, structured mesh. The various meshes used in this section are presented in Figure 4.15.

The meshes include orthogonal structured quadrilateral meshes and non-orthogonal structured meshes that are perturbed through a random process. The perturbation involves a recursive algorithm that decomposes a quadrilateral into four quadrilaterals. Each of these quadrilaterals is in turn decomposed into four more quadrilaterals. The midpoint of the quadrilateral is perturbed using a random number. This perturbation is a function of the parameter $f$ shown in (4.23).

$$
\begin{array}{r}
x^{\text {new }}=s x^{\text {node } i}+(1-s) x^{\text {node } j} \\
y^{\text {new }}=s y^{\text {node } i}+(1-s) y^{\text {node } j} \\
s=f+(1-2 f) \operatorname{rand}(\cdot)
\end{array}
$$

This algorithm is applied to each edge of the quadrilateral defined by node $i$ and node $j$. The value $\operatorname{rand}(\cdot)$ is a random number between 0 and 1 . The four new nodes are used to find the center of the quadrilateral being partitioned. Once the center is found the four nodes are connected to the central node forming four new sub quadrilaterals.

When $f$ is 0.5 , no perturbation occurs and the mesh is an orthogonal structured mesh as shown in the top left corner of Figure 4.15. When the parameter $f$ increases the mesh becomes nonorthogonal as shown for various cases of $f$. The mesh size is a 
Grid size $=$ H33, 33L $f=0.5$

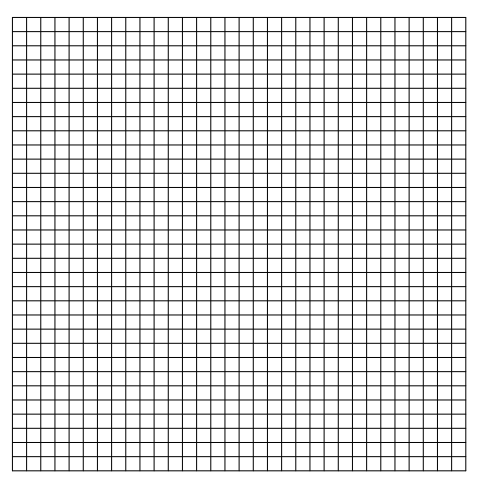

Grid size $=H 33,33 \mathrm{~L} f=0.47$

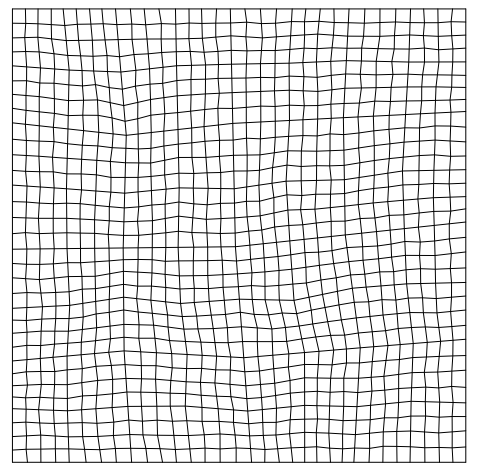

Grid size $=$ H33, 33L $f=0.35$

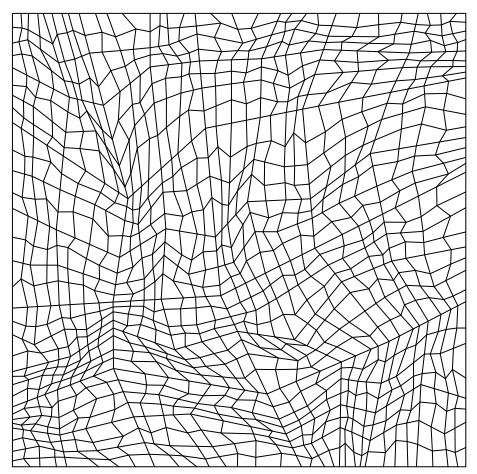

Grid size $=H 33,33 \mathrm{~L} f=0.49$

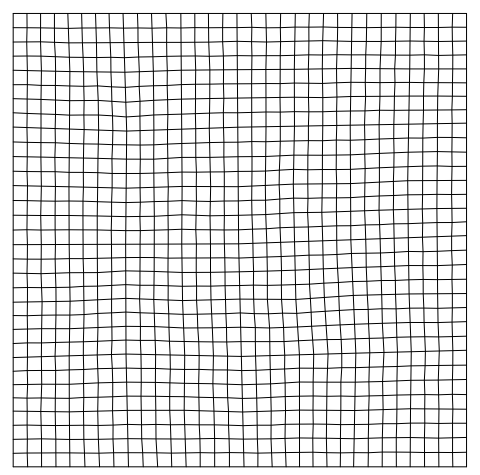

Grid size $=$ H33, 33L $\mathrm{f}=0.44$

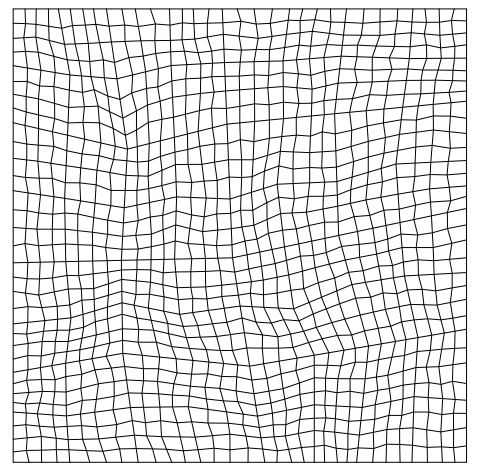

Figure 4.15: 2D Quadrilateral Meshes 
factor of 2 and is given by $2^{k-1}+1 x 2^{k-1}+1$.

The two-dimensional edge basis functions will be used to characterize the mass matrix solution for edge elements. These basis functions for a quadrilateral element are given in Table 4.1. The mass and stiffness matrices for these 1-form basis functions

Table 4.1: Two dimensional edge basis functions for a Quadrilateral

\begin{tabular}{|c|c|c|}
\hline Basis & \multicolumn{2}{|l|}{$W$} \\
\hline 1 & $\frac{1}{4}(1-\psi)$ & \begin{tabular}{|l|l|} 
\\
\end{tabular} \\
\hline 2 & $\frac{1}{4}(1+\psi)$ & $\begin{array}{l}1 \\
0\end{array}$ \\
\hline 3 & $\frac{1}{4}(1-\xi)$ & 0 \\
\hline 4 & $\frac{1}{4}(1+\xi)$ & {$\left[\begin{array}{l}1 \\
0\end{array}\right]$} \\
\hline
\end{tabular}

can be formed as in Chapter 3 resulting in the discrete electric field wave equation (4.24). A four-point Gaussian quadrature rule was used for the inner product integrations.

$$
\mathbf{M}_{1} \ddot{\vec{e}}=-\mathbf{K}_{1} \vec{e}
$$

The mass matrix can be characterized by various metrics. For solution scaling the most important of these is the condition number. The two-dimensional edge mass matrix condition number for various meshes with $n$ total edges are shown in Table 4.2.

Table 4.2: Condition number of $2 \mathrm{D}$ edge element mass matrix

\begin{tabular}{|c|c|c|c|c|c|}
\hline$n$ & $f=0.5$ & $f=0.49$ & $f=0.47$ & $f=0.44$ & $f=0.35$ \\
\hline \hline 144 & 3.92 & 3.86 & 4.41 & 5.27 & 10.31 \\
\hline 544 & 3.97 & 3.92 & 4.95 & 6.76 & 20.28 \\
\hline 2112 & 4.00 & 3.97 & 5.49 & 8.35 & 43.35 \\
\hline 5320 & 4.00 & 3.99 & 6.15 & 10.69 & 86.18 \\
\hline 33024 & 4.07 & 4.00 & 7.20 & 15.72 & 230.0 \\
\hline
\end{tabular}


The condition number for orthogonal meshes and slightly perturbed meshes is constant, but as the perturbation grows the mass matrix becomes more ill-conditioned as the size grows. In Table 4.3 the condition number of the Mass matrix under diagonal preconditioning is improved over the non-preconditioned mass matrix.

Table 4.3: Condition number of 2D edge element diagonally preconditioned system

\begin{tabular}{|c|c|c|c|c|}
\hline$n$ & $f=0.49$ & $f=0.47$ & $f=0.44$ & $f=0.35$ \\
\hline \hline 40 & 3.00 & 3.15 & 3.33 & 4.08 \\
\hline 144 & 3.00 & 3.18 & 3.43 & 4.43 \\
\hline 544 & 3.00 & 3.28 & 3.64 & 5.58 \\
\hline 2112 & 3.00 & 3.43 & 3.95 & 10.87 \\
\hline 5320 & 3.00 & 3.63 & 4.30 & 16.28 \\
\hline
\end{tabular}

The number of iterations necessary for the solution convergence for one time step of (4.24) of the mesh with $f=0.44$ are shown in Table 4.4. The preconditioners used were Jacobi-diagonal scaling and incomplete-LU.

Table 4.4: Number of conjugate gradient iterations for $f=0.44$

\begin{tabular}{|c|c|c|}
\hline$n$ & Jacobi & ILU \\
\hline \hline 144 & 14 & 5 \\
\hline 544 & 15 & 6 \\
\hline 2112 & 16 & 6 \\
\hline 33024 & 16 & 7 \\
\hline
\end{tabular}

The condition number for the preconditioned system with $f=0.35$ in Table 4.3 does not approach a constant as in the other cases. Unlike uniform grids the fundamental structure of this non-uniform grid is not constant as the number of grid points is increased. This is evident when mesh metrics such as the ratio of maximum to minimum zone size, Table 4.5, and the ratio of maximum to minimum edge lengths, Table 4.6, are compared for the different meshes. 
Table 4.5: Ratio of maximum to minimum zone area for various grid sizes

\begin{tabular}{|c|c|c|c|}
\hline$n$ & $f=0.47$ & $f=0.44$ & $f=0.35$ \\
\hline \hline 40 & 0.86 & 0.72 & 0.44 \\
\hline 144 & 0.77 & 0.59 & 0.24 \\
\hline 544 & 0.69 & 0.47 & 0.12 \\
\hline 2112 & 0.62 & 0.37 & 0.07 \\
\hline 5320 & 0.54 & 0.29 & 0.03 \\
\hline 33024 & 0.48 & 0.23 & 0.01 \\
\hline
\end{tabular}

Table 4.6: Ratio of maximum to minimum edge length for various grid sizes

\begin{tabular}{|c|c|c|c|}
\hline$n$ & $f=0.47$ & $f=0.44$ & $f=0.35$ \\
\hline \hline 40 & 0.856 & 0.733 & 0.441 \\
\hline 144 & 0.796 & 0.626 & 0.293 \\
\hline 544 & 0.719 & 0.509 & 0.172 \\
\hline 2112 & 0.657 & 0.424 & 0.107 \\
\hline 5320 & 0.597 & 0.351 & 0.069 \\
\hline 33024 & 0.547 & 0.294 & 0.042 \\
\hline
\end{tabular}

To determine if a result holds that is similar to the Ciarlet Theorem [40] for nodal finite elements, the previous computations were carried out on a sequence of grids whose diameters are related, Figure 4.16. Ciarlet's theorem involves the nodal mass matrix and states that if a sequence of meshes conforms to the criteria in Ciarlet's theorem, then the conjugate gradient method will attain a constant number of iterations as the number of nodes is increased. The initial coarse grid was constructed using a seed of $f=0.35$. This grid is then partitioned to create a family of related grids. The mesh metrics for this family of meshes are listed in Table 4.7,Table 4.8 and Table 4.9.

The condition number of the diagonally preconditioned edge mass matrix remains essentially constant as the size of the grid increases provided the ratio of the edge lengths remains constant. This is useful when the preconditioned conjugate gradient 
Grid size $=$ H9, 9L $f=0.35$

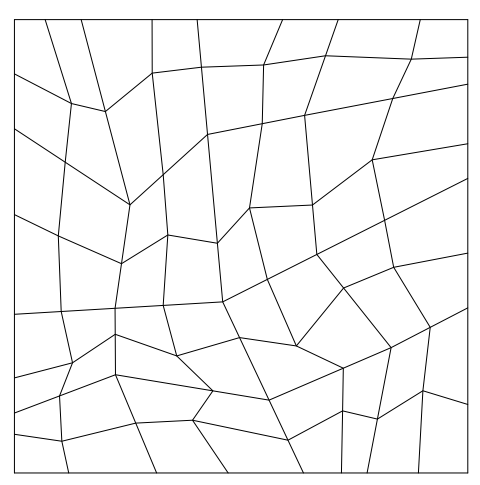

Grid size $=H 33,33 \mathrm{~L} f=0.35$

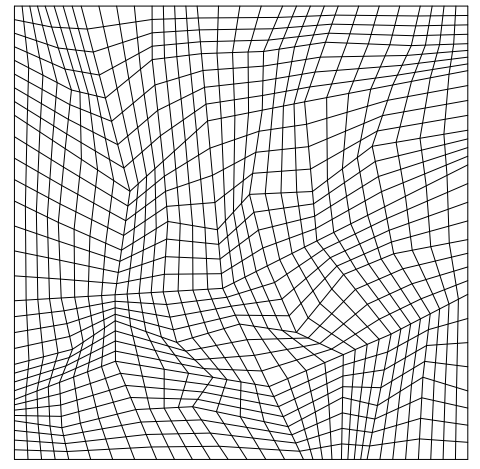

Grid size $=$ H17, 17 L $f=0.35$

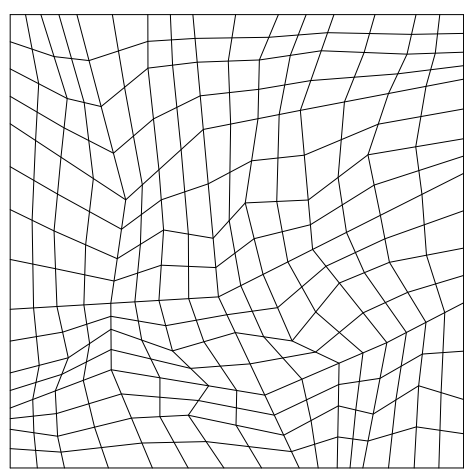

Grid size $=H 65,65 \mathrm{~L} \quad \mathrm{f}=0.35$

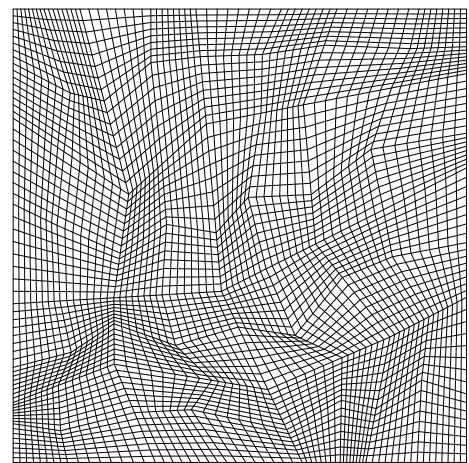

Figure 4.16: 2D Quadrilateral Refinement Meshes

Table 4.7: Mesh Metrics for refined mesh sequence $f=0.35$

\begin{tabular}{|c|c|c|}
\hline$m$ & $\frac{\max (\text { area })}{\min (\text { area })}$ & $\frac{\max (\text { diam })}{\min (\text { diam })}$ \\
\hline \hline 4 & 0.237794 & 0.293104 \\
\hline 5 & 0.217542 & 0.293099 \\
\hline 6 & 0.207517 & 0.293091 \\
\hline 7 & 0.197316 & 0.293072 \\
\hline 8 & 0.192447 & 0.293035 \\
\hline
\end{tabular}


Table 4.8: Condition number for mass matrix and diagonally preconditioned mass matrix for refined mesh sequence $f=0.35$

\begin{tabular}{|c|c|c|}
\hline$m$ & $\kappa\left(\mathbf{M}_{1}\right)$ & $\kappa\left(\mathbf{Q}^{-1} \mathbf{M}_{1}\right)$ \\
\hline \hline 4 & 20.29 & 4.44 \\
\hline 5 & 25.32 & 4.56 \\
\hline 6 & 30.05 & 4.71 \\
\hline 7 & 31.40 & 4.91 \\
\hline 8 & 36.75 & 5.17 \\
\hline
\end{tabular}

Table 4.9: Condition number for diagonally scaled conj. grad. and ILU conj. grad. for refined mesh sequence $f=0.35$

\begin{tabular}{|c|c|c|}
\hline$m$ & Jacobi & ILU \\
\hline \hline 4 & 17 & 7 \\
\hline 5 & 17 & 8 \\
\hline 6 & 18 & 9 \\
\hline 7 & 18 & 9 \\
\hline 8 & 18 & 9 \\
\hline
\end{tabular}

method is used for the system solution. 


\section{Chapter 5}

\section{Numerical Method Analysis}

The discrete differential forms method outlined in the previous chapter defines the spatial discretization. In this chapter the time integration method and its convergence along with numerical dispersion and conservation will be analyzed.

The time integration for the second order wave equation simulations in this dissertation is a second order accurate leap frog method (5.1).

$$
\frac{\partial^{2} x}{\partial t^{2}}=\frac{x^{n+1}-2 x^{n}+x^{n-1}}{\Delta t^{2}}+\mathcal{O}\left(\Delta t^{2}\right)
$$

The first order equations also use the second order accurate leap-frog time integration $(5.2)$.

$$
\frac{\partial x}{\partial t}=\frac{x^{n+\frac{1}{2}}-x^{n-\frac{1}{2}}}{\Delta t}+\mathcal{O}\left(\Delta t^{2}\right)
$$

This time integration approximation will converge to the true solution as $\delta t \rightarrow 0$ if it meets the conditions of consistency and stability [48]. Consistency is a measure of how well the finite difference equation (FDE) approximates the partial differential equation (PDE). The FDE is consistent if as the mesh is refined the truncation error, the error between the continuous PDE and the discrete FDE, goes to zero. The discrete wave 
equations with no loss terms can all be written in the form (5.3) where $\mathbf{M}$ is the mass matrix which refines as $\Delta x$ and $\mathbf{K}$ is the stiffness matrix which refines as $\frac{1}{\Delta x}$. As the mesh is refined both $\Delta x$ and $\Delta t$ grow smaller which gives $\lim _{\Delta x \rightarrow 0, \Delta t \rightarrow 0} \frac{\Delta t^{2}}{\Delta x^{2}}=0$. The time step $\Delta t \propto \Delta x$ therefore they grow smaller at approximately the same rate resulting in a consistent method.

$$
\mathbf{x}^{n+1}=-\Delta t^{2} \mathbf{M}^{-1} \mathbf{K}+2 \mathbf{x}^{n}-\mathbf{x}^{n-1}
$$

Convergence requires not only consistency but stability. Stability is a measure of error growth as a time marching algorithm progresses. If the errors (round-off, truncation error, bad design) do not grow in time the method is stable. Each wave equation will be analyzed for stability in the sections below.

Dispersion is the effect in which waves at different frequencies will propagate at different phase velocities. The phase velocity is defined as the ratio of the angular frequency $\omega=2 \pi \nu$ to the wave number $k=|\vec{k}|=\frac{2 \pi}{\lambda}$ giving $v_{p}=\frac{\omega}{k}$ where $\nu$ and $\lambda$ are the frequency and wavelength of the wave. The quantity $\vec{k}$ is the wave vector, this vector points in the direction of propagation. Dispersion can result in a narrow Gaussian pulse spreading out due to the dissimilar propagation speeds of the Fourier components. The phase velocity can also depend on the wave vector $\vec{k}$, this results in waves propagating at different velocities in different directions.

Numerical Dispersion [49], [50], [51] is the effect of dispersion due to a the discrete nature of the simulation. In each wave equation section in this chapter a numerical dispersion relation will be derived. In all cases it will be shown that the numerical dispersion is accurate to second order. The derivation of the numerical dispersion relation proceeds in the same way for all of the wave equations. The wave equation is 
written with no loss terms and constant coefficients and a scalar or vector plane wave solution is assumed. The degrees of freedom on a hexahedral zone will differ by a phase constant from a chosen set of degrees of freedom. These degrees of freedom are then entered into the discrete form of the wave equation and solved for the dispersion relation.

This chapter also includes the analysis of numerical conservation in the method. The term numerical conservation describes the link between conserved mathematical representations and their corresponding physical representations. Just as energy, charge and vorticity are conserved in nature so should they be in the method. The following sections describe the mimetic nature of the method with respect to conservation.

\subsection{Scalar Wave Equation}

To analyze the scalar wave equation the discrete representation will be written with constant coefficients and without any loss terms in the natural (5.4) and adjoint (5.5) forms.

$$
\begin{gathered}
\mathbf{M}_{0} \ddot{\mathbf{x}}=-\mathbf{K}_{0} \mathbf{x} \\
\mathbf{M}_{3} \ddot{\mathbf{x}}=-\mathbf{M}_{3} \mathbf{D M}_{2}^{-1} \mathbf{D}^{T} \mathbf{M}_{3} \mathbf{x}
\end{gathered}
$$

\subsubsection{Numerical stability}

The analysis of the stability for a three time level numerical algorithm proceeds by rewriting the algorithm as a two level algorithm. This is accomplished by using 
an auxiliary variable. In the case of the scalar wave equation the result is shown in (5.6).

$$
\begin{aligned}
& \mathbf{x}^{n+1}=2 \mathbf{I}-(\Delta t)^{2} \mathbf{M}_{0}^{-1} \mathbf{K}_{0} \mathbf{x}^{n}-\mathbf{z}^{n} \\
& \mathbf{z}^{n+1}=\mathbf{x}^{n}
\end{aligned}
$$

which becomes :

$$
\left[\begin{array}{c}
\mathbf{x}^{n+1} \\
\mathbf{z}^{n+1}
\end{array}\right]=\left[\begin{array}{cc}
\mathbf{B} & -\mathbf{I} \\
\mathbf{I} & 0
\end{array}\right]\left[\begin{array}{l}
\mathbf{x}^{n} \\
\mathbf{z}^{n}
\end{array}\right]
$$

where $\mathbf{B}=2 \mathbf{I}-(\Delta t)^{2} \mathbf{M}_{0}^{-1} \mathbf{K}_{0}$. Rewriting the equation in this way forms a simple discrete representation given in (5.8)

$$
\mathbf{u}^{n+1}=\mathbf{Q u}^{n}
$$

where $\mathbf{u}$ is given by $\mathbf{u}^{n}=\left[\begin{array}{l}\mathbf{x}^{n+1} \\ \mathbf{z}^{n+1}\end{array}\right]$. Determining the stability for this equation depends on whether $\mathbf{Q}$ will amplify, attenuate or not affect the error. A necessary criteria for stability is the two-norm of $\mathbf{Q}$ must be less than or equal to $1,\|\mathbf{Q}\| \leq 1$. The spectral radius $\rho(\mathbf{Q})$, maximum absolute eigenvalue of $\mathbf{Q}$, is related to $\|\mathbf{Q}\|$ by (5.9). A necessary but not sufficient condition for stability is $\rho(\mathbf{Q}) \leq 1$.

$$
\rho(\mathbf{Q}) \leq\|\mathbf{Q}\| \leq 1
$$

Evaluating $\rho(\mathbf{Q})$ leads to (5.10).

$$
\lambda=\frac{-\delta \pm \sqrt{\delta^{2}-4}}{2}
$$

where $\delta$ is the maximum eigenvalue of $\mathbf{B}$. Spectral stability is attained if $\lambda \leq 1$, solving for $\delta$ gives (5.11).

$$
\delta=-2
$$


In (5.4) the matrix $\mathbf{M}_{0}$ is symmetric positive definite and $\mathbf{K}_{0}$ is symmetric positive semi-definite. This leads to the result that for $\delta=2-(\Delta t)^{2} \kappa_{0}$, the eigenvalues $\kappa_{0} \geq 0$ where $\kappa_{0}$ are the eigenvalues represented in (5.12).

$$
\mathbf{M}_{0} \mathbf{x}=-\kappa_{0} \mathbf{K}_{0} \mathbf{x}
$$

Substituting the expression for $\delta$ into (5.10) we see the eigenvalues of $\mathbf{Q}$ will only have unit magnitude given (5.13).

$$
\Delta t \leq \frac{2}{\sqrt{\max \left(\kappa_{0}\right)}}
$$

The result in (5.13) is a necessary but not sufficient condition for stability. Three types of stability can be defined. The first describes a system with eigenvalues all within the unit circle and a complete set of linearly independant eigenvectors, called strong stability. The second is called neutrally stable which describes a system with eigenvalues on the unit circle and a complete set of linearly independant eigenvectors. The third type is called weakly stable and describes a system with eigenvalues on or within the unit circle and an incomplete set of linearly independant eigenvectors. The eigenvectors of the amplification matrix $\mathbf{Q}$ must all be linearly independent for stability with no growth. The case of the second order wave equation with Neumann boundary conditions does not meet the criteria for stability with no growth. Stability can also be defined as a solution that only grows polynomially not exponentially. The second order wave equation grows, at worst, linearally in time. Linear growth results due to a valid unphysical solution $\phi=c t$, where $\mathrm{c}$ is a constant, of the second order wave equation.

The growth of the amplification system can be bounded by looking at the eigen- 
vectors and eigenvalues of the matrix $\mathbf{Q}$. If $\mathbf{x}$ is an eigenvector of the system (5.12), then the amplification system becomes (5.14).

$$
\mathbf{Q}\left[\begin{array}{c}
\mathbf{x} \\
\mathbf{z}
\end{array}\right]=\mathbf{Q}\left[\begin{array}{c}
\mathbf{x} \\
\frac{1}{\lambda} \mathbf{x}
\end{array}\right]=\lambda\left[\begin{array}{c}
\mathbf{x} \\
\frac{1}{\lambda} \mathbf{x}
\end{array}\right]
$$

If $\mathbf{x}$ is an eigenvector in the range of $\mathbf{A}=\mathbf{M}_{0}^{-1} \mathbf{K}_{0}$ then the amplification system will oscillate but not grow. If the vector $\mathbf{x}$ is in the null space of $\mathbf{A}$ then the system will have an eigenvalue $\lambda=1$ with eigenvector (5.15) and will grow no faster than linearly.

$$
\left[\begin{array}{l}
\mathbf{x} \\
\mathbf{x}
\end{array}\right]
$$

The linear growth can be shown by creating the Jordan form of the matrix $\mathbf{Q}=$ $\mathbf{V}^{-1} \mathbf{J V}$. The matrix $\mathbf{J}$ consists of Jordan blocks which are $k$-dimensional blocks with the eigenvalues on the diagonal and ones on the super-diagonal. The size of the Jordan blocks can be determined by solving the system (5.16).

$$
(\mathbf{Q}-\lambda \mathbf{I})^{k} \mathbf{u}=0
$$

For the amplification system with eigenvector (5.15) the integer $k=2$. This means that there is no Jordan block greater than $2 \times 2$. The dimension of the Jordan block is related to the norm of $\mathbf{Q}$ through the minimum polynomial of degree $k$. Since $k$ can be no larger than 2 , the minimum polynomial for the system is of the form $\alpha x+\beta$. When the amplification matrix is applied many times the norm of $\mathbf{Q}$ will be related to $\mathbf{J}$ through $\mathbf{Q}^{n}=\mathbf{V}^{-1} \mathbf{J}^{n} \mathbf{V}$. Therefore the amplification system will grow no greater than linearly. 
To demonstrate the various solutions that may result and their growth, the eigenvalues and eigenvectors of the matrix $\mathbf{Q}$ resulting from a small hexahedral sphere grid were computed. Table 5.1 contains the grid statistics for the hexahedral sphere. The space dimensions for the natural Div-Grad operator are shown in Table 5.2.

Iterating $\mathbf{u}^{n+1}=\mathbf{Q u}^{n}$ for a valid static, valid dynamic and invalid solution results in the figures shown along with the eigenvalue spectrum in Figure 5.1. The static solution was created by using one of the null space eigenvectors, in this case the constant vector. The dynamic solution was created by creating the rectangular matrix of valid (non-zero) eigenvectors $\mathbf{L}$ and creating the projection matrix $\mathbf{P}=\mathbf{L L}^{T}$. The initial solution vector is then projected onto this space using the matrix $\mathbf{P}$. The invalid solution is created by adding random noise into the valid dynamic solution. The invalid solution increases, at worst, linearly resulting in a weakly stable system. It is important to note that the solution $\phi=c t$ is not a valid physical solution, but is a valid solution of the P.D.E. The discretization is modelling the P.D.E. accurately including the non-physical solution.

Table 5.1: Statistics for the hexahedral sphere grid.

\begin{tabular}{|c|c|c|c|}
\hline Type & Total & Internal & Boundary \\
\hline \hline zones & 56 & & \\
\hline edges & 202 & 154 & 48 \\
\hline faces & 180 & 156 & 24 \\
\hline nodes & 79 & 53 & 26 \\
\hline
\end{tabular}

The adjoint form of the scalar wave equation has a stability condition similar to (5.9). The matrix $\mathbf{B}$ is equal to (5.17).

$$
\mathbf{B}=2 \mathbf{I}-(\Delta t)^{2} \mathbf{M}_{3}^{-1} \mathbf{M}_{3} \mathbf{D} \mathbf{M}_{2}^{-1} \mathbf{D}^{T} \mathbf{M}_{3}
$$


Table 5.2: Space dimensions for the natural Div-Grad operator test equations on a hexahedral sphere grid.

\begin{tabular}{|c|c|c|c|c|}
\hline Operator & $\mathrm{n}$ & $2 \mathrm{n}$ & Distinct Eigenvalues & Deg. Eigenvalues \\
\hline \hline Div-Grad & 79 & 158 & 157 & 1 \\
\hline
\end{tabular}
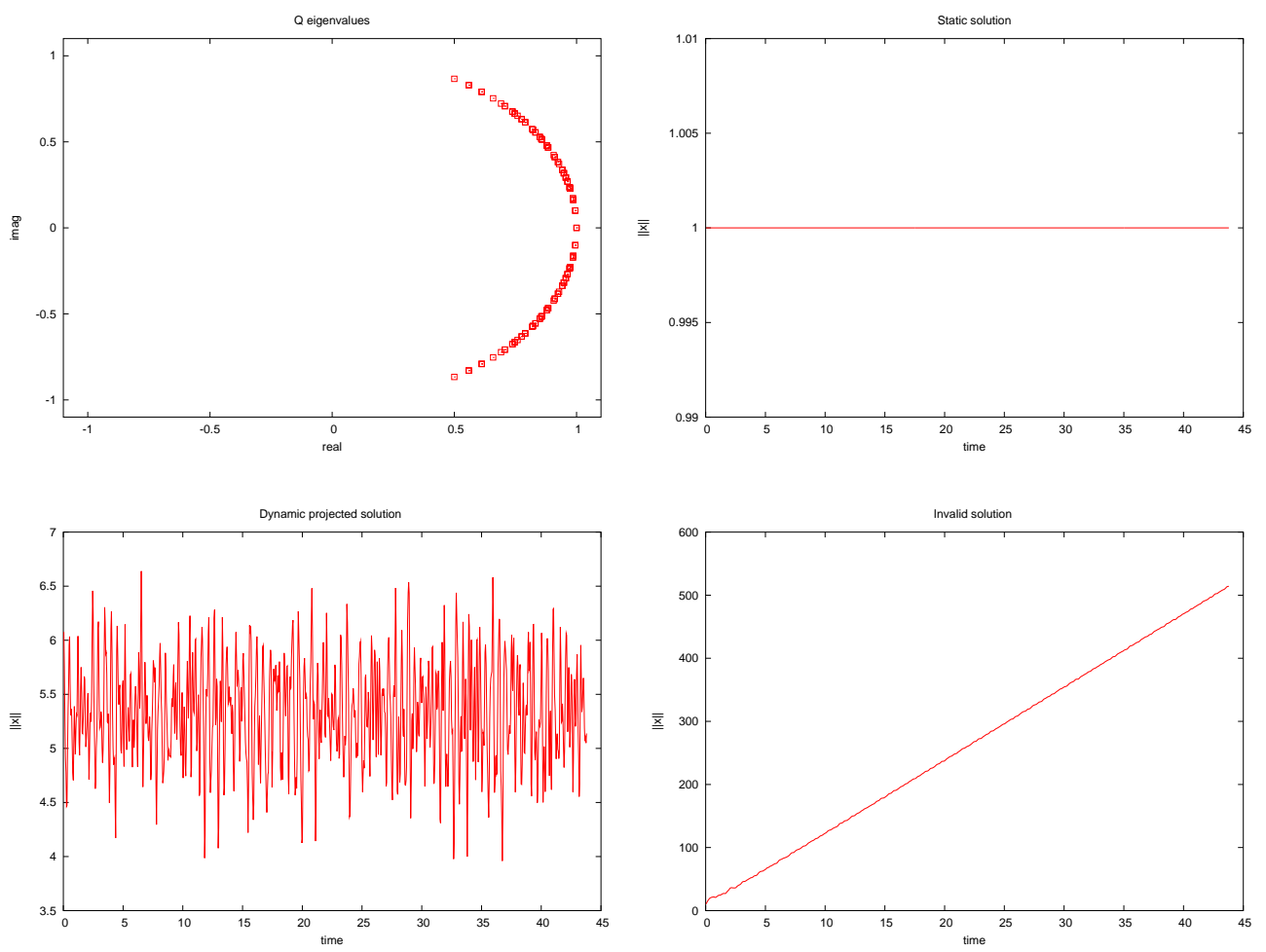

Figure 5.1: Second order natural Div-Grad solutions 
In this case the eigenvalues $\kappa_{3}$ come from the equation (5.18).

$$
\mathbf{M}_{3} \mathbf{x}=-\kappa_{3} \mathbf{M}_{3} \mathbf{D M}_{2}^{-1} \mathbf{D}^{T} \mathbf{M}_{3} \mathbf{x}
$$

In this case both the mass matrix $\mathbf{M}_{3}$ and the stiffness matrix (5.19) are symmetric positive definite so $\kappa_{3} \geq 0$.

$$
\mathbf{M}_{3} \mathbf{D M}_{2}^{-1} \mathbf{D}^{T} \mathbf{M}_{3}
$$

The stability condition becomes (5.20).

$$
\Delta t \leq \frac{2}{\sqrt{\max \left(\kappa_{3}\right)}}
$$

In this case the operator is symmetric positive definite and therefore has linearly independent eigenvectors meeting the criteria for stability.

Because the operator with essential boundary conditions corresponds to an operator with no null-space the space dimensions show no degenerate eigenvalues for the operator in Table 5.3. The eigenvalue spectrum, valid dynamic and "invalid" solutions are shown in Figure 5.2.

Table 5.3: Space dimensions for the adjoint Div-Grad operator test equations on a hexahedral sphere grid

\begin{tabular}{|c|c|c|c|c|}
\hline Operator & $\mathrm{n}$ & $2 \mathrm{n}$ & Distinct Eigenvalues & Deg. Eigenvalues \\
\hline \hline Div-Grad & 56 & 112 & 112 & 0 \\
\hline
\end{tabular}

\subsubsection{Numerical dispersion}

The numerical dispersion relation for the natural form of the scalar wave equation for pressure is derived by choosing a plane wave solution (5.21).

$$
P_{1}(\vec{r}, t)=\tilde{P} e^{i(\vec{k} \cdot \vec{r}-\omega t)}
$$



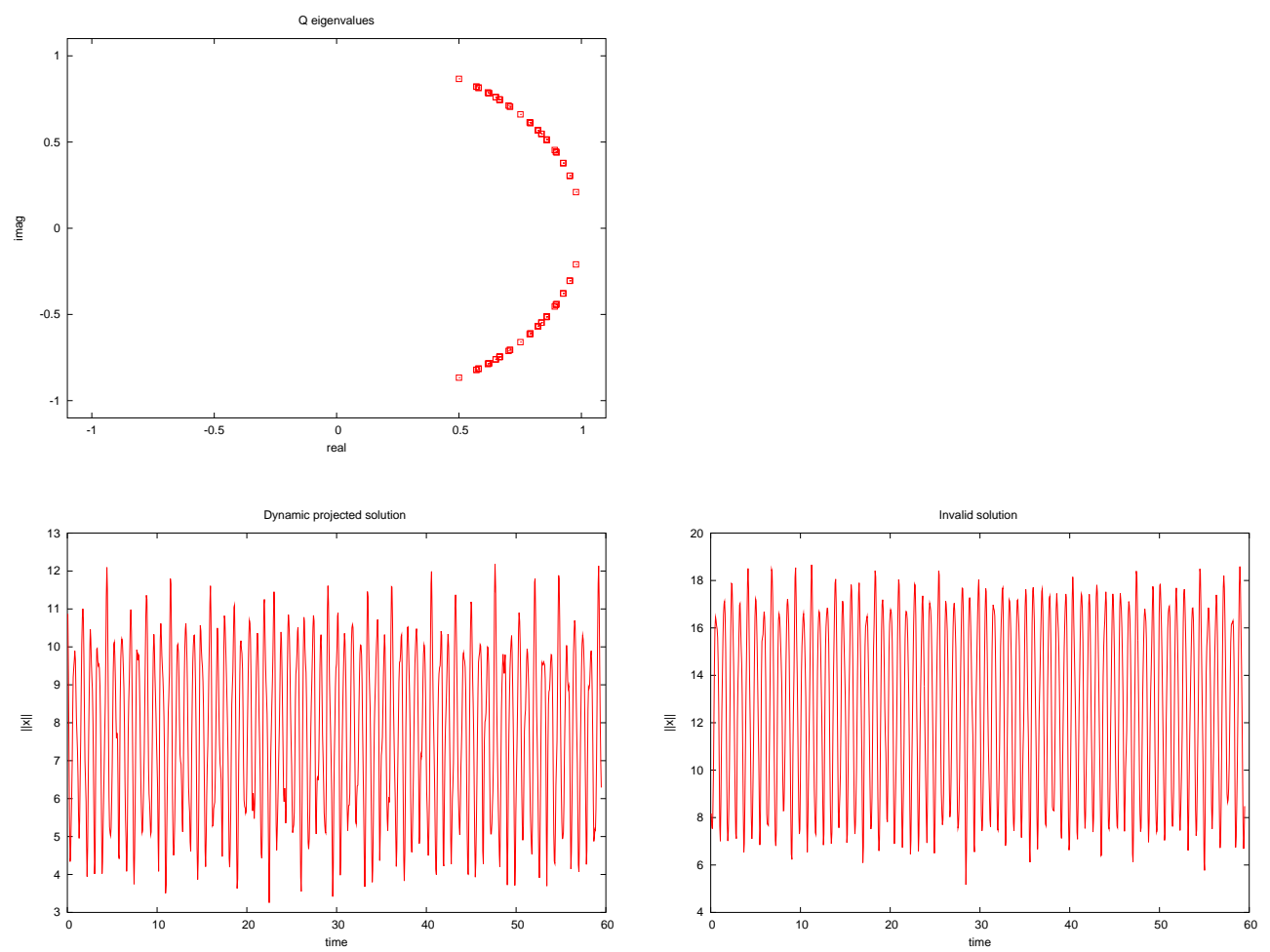

Figure 5.2: Second order adjoint Div-Grad solutions 
The dispersion relation for the plane wave solution is given by (5.22)

$$
\frac{\omega^{2}}{k^{2}}=c_{l}^{2}
$$

Due the the finite sampling of time and space in the simulation numerical dispersion will result. This analysis will show that the order of accuracy due to errors introduced by numerical dispersion is second order. The dispersion for the discrete pressure scalar wave equation with constant coefficients (5.23) will be determined for a three dimensional hexahedral region of space.

$$
\mathbf{M}_{0} \frac{\partial^{2} \vec{\beta}}{\partial t^{2}}=-\mathbf{K}_{0} \vec{\beta}
$$

The degrees of freedom $\vec{\beta}$ are the pressures located at the nodes and $\mathbf{M}_{0}$ and $\mathbf{K}_{0}$ are the 0 -form mass and stiffness matrices respectively presented previously. Referring to the node numbering scheme in Figure 3.11, the first node $n_{1}$ will be given a value of $\alpha$. All of the other nodes will differ from this value by a phase difference $\overrightarrow{\Delta x_{i}}$, where $i$ is the node number, and a time difference $\Delta t$. The value of $\overrightarrow{\Delta x_{i}}$ is the vector from node $n_{1}$ to node $n_{i}$. The resulting nodal degrees of freedom are given in Table 5.4.

Table 5.4: Nodal Dispersion Degrees Of Freedom

\begin{tabular}{|c|c|}
\hline DOF & Function \\
\hline \hline$\beta_{1}$ & $\alpha$ \\
\hline$\beta_{2}$ & $\alpha e^{-\left(\vec{k} \cdot \Delta x_{2}-\omega \Delta t\right)}$ \\
\hline$\beta_{3}$ & $\alpha e^{-\left(\vec{k} \cdot \Delta x_{3}-\omega \Delta t\right)}$ \\
\hline$\beta_{4}$ & $\alpha e^{-\left(\vec{k} \cdot \Delta x_{4}-\omega \Delta t\right)}$ \\
\hline$\beta_{5}$ & $\alpha e^{-\left(\vec{k} \cdot \Delta x_{5}-\omega \Delta t\right)}$ \\
\hline$\beta_{6}$ & $\alpha e^{-\left(\vec{k} \cdot \Delta x_{6}-\omega \Delta t\right)}$ \\
\hline$\beta_{7}$ & $\alpha e^{-\left(\vec{k} \cdot \Delta x_{7}-\omega \Delta t\right)}$ \\
\hline$\beta_{8}$ & $\left.\alpha e^{-(\vec{k} \cdot \overrightarrow{\Delta x}}-\omega \Delta t\right)$ \\
\hline
\end{tabular}

Entering the plane wave solution (5.21) into the time derivative and approximating 
using the second order time differentiation scheme (5.1) gives (5.24).

$$
\begin{array}{r}
\frac{\partial^{2} \beta_{i}}{\partial t^{2}} \approx \frac{\beta_{i}^{n+1}-2 \beta_{i}^{n}+\beta_{i}^{n-1}}{\Delta t^{2}}=\frac{\Psi \beta_{i}}{\Delta t^{2}} \\
\Psi=\frac{2}{\Delta t^{2}}(\cos (\omega \Delta t)-1)
\end{array}
$$

The degrees of freedom entered into (5.23) along with the time derivative approximation (5.24) gives the residual equation (5.26) where $\nu=c_{l}^{2} \frac{\Delta t^{2}}{\Delta h^{2}}$. This equation is derived through the variational equation. If the plane wave solution is a true solution the residual will be zero. This equation can be solved for the single root $R$ which will be a relation between $\omega$ and $\vec{k}$.

$$
\left(\Psi \mathbf{D m}_{0}+\nu \mathbf{D k}_{0}\right)(\alpha)=0
$$

The general solution for the root $R$ Taylor expanded around $\delta h=0$ is given in $(5.27)$

$$
R_{t e}=k^{2}+\mathcal{O}\left(\Delta h^{2}\right)
$$

Choosing a wave vector on a uniform Cartesian grid $\vec{k}=a \hat{x}+b \hat{y}+c \hat{z}$ and Taylor expanding the root about $\Delta h=0$.

$$
R_{t e}=k^{2}\left(1+\frac{1}{12}(k \Delta h)^{2}+\mathcal{O}\left(\Delta h^{4}\right)\right)
$$

The root $R$ Taylor expanded around $\Delta t=0$ is given in (5.29).

$$
R_{t e}=\omega^{2}\left(1-\frac{1}{12}(\omega \Delta t)^{2}+\mathcal{O}\left(\Delta t^{4}\right)\right)
$$

Combining the two Taylor expansions, the generalized dispersion relation for the 0form scalar wave equation is shown to be second order accurate (5.30).

$$
\frac{\omega^{2}}{k^{2}}=c_{l}^{2} \frac{\left(1+\frac{1}{12}(k \Delta h)^{2}+\mathcal{O}\left(\Delta h^{4}\right)\right)}{\left(1-\frac{1}{12}(\omega \Delta t)^{2}+\mathcal{O}\left(\Delta t^{4}\right)\right)}
$$


The adjoint form of the Laplacian for 3-forms can be analyzed in a similar manner as the 0 -forms above. The plane wave of the form (5.21) is again used, but in the case the discrete operator equation is given by (5.31).

$$
\beta=-\mathbf{D M}_{2}^{-1} \mathbf{D}^{T} \mathbf{M}_{3} \beta
$$

The time and spatial domains are analyzed separately. The second order time derivative results in (5.24). This equation can be Taylor expanded in order to derive the denominator of (5.30). The expansion is exactly the same for the 3 -form due to the same time integration method.

Using the operator stencil given by Figure 4.14 centered on the origin the sum in (5.32) is performed.

$$
\phi=\sum_{i}^{n} c e^{\left(-i \vec{k} \cdot \Delta x_{i}\right)}
$$

This sum is then Taylor expanded in terms of the three components of the wave

vector $\vec{k}$. The resulting relation will be the leading $|\vec{k}|^{2}$ term followed by the spatial dispersion truncation error. The result is shown in (5.33).

$$
\frac{\omega^{2}}{k^{2}}=c_{l}^{2} \frac{\left(1+\frac{1}{12}(k \Delta h)^{2}+\mathcal{O}\left(\Delta h^{4}\right)\right)}{\left(1-\frac{1}{12}(\omega \Delta t)^{2}+\mathcal{O}\left(\Delta t^{4}\right)\right)}
$$

\subsubsection{Energy conservation}

The energy for the acoustic wave equation is given in (2.22). To show the conservation of energy the integrodifferential form is used (5.34)

$$
\oint_{\Gamma} P_{1} \vec{v} \cdot \hat{n} d \Gamma+\int_{\Omega} \rho_{0} \vec{v}_{1} \cdot \frac{\partial \vec{v}_{1}}{\partial t}+\left(\gamma P_{0}\right)^{-1} P_{1} \frac{\partial P_{1}}{\partial t}=0
$$

The first term represents the energy flowing into the domain while the second and third terms represent the time rate of change of the potential and kinetic energy 
respectively. Using the 0 -form and 1-form mass matrices $\mathbf{M}_{0}$ and $\mathbf{M}_{1}$ for the pressure term and velocity term respectively the discrete version of (5.34) can be derived and is shown in (5.35). In this equation the energy flowing into the domain is set to be zero. The 0 -form and 1-form mass matrices have materials given by $\delta=\left(\gamma P_{0}\right)^{-1}$ and $\nu=\rho_{0}$ respectively.

$$
\alpha^{T} \mathbf{M}_{0}^{\delta} \frac{\partial \alpha}{\partial t}+\beta^{T} \mathbf{M}_{1}^{\alpha} \frac{\partial \beta}{\partial t}=0
$$

Using the leap frog integration scheme for the single time derivative equations (5.2) in (5.35) the energy equation (5.36) can be shown to conserve energy in a time averaged sense.

$$
\left(\alpha^{n+1}\right)^{T} \mathbf{M}_{0}^{\delta} \alpha^{n+1}+\left(\beta^{n+\frac{1}{2}}\right)^{T} \mathbf{M}_{1}^{\nu} \beta^{n+\frac{1}{2}}+\left(\alpha^{n}\right)^{T} \mathbf{M}_{0}^{\delta} \alpha^{n}+\left(\beta^{n-\frac{1}{2}}\right)^{T} \mathbf{M}_{1}^{\nu} \beta^{n-\frac{1}{2}}=0
$$

This result is derived through an analysis of the first order equations as done with the second order equations in the stability section. The result of the first order stability analysis is the same as the second order result (5.13). The amplification matrix for the first order equations also has eigenvalues with unit magnitude. Using this fact the energy equation is constant in time and therefore conserves energy.

The adjoint scalar wave equation energy analysis is performed in exactly the same manner. Making the change to a 2-form, 3-form formulation results in the discrete energy equation for the adjoint case (5.37).

$$
\left(\alpha^{n+1}\right)^{T} \mathbf{M}_{3}^{\delta} \alpha^{n+1}+\left(\beta^{n+\frac{1}{2}}\right)^{T} \mathbf{M}_{2}^{\nu} \beta^{n+\frac{1}{2}}+\left(\alpha^{n}\right)^{T} \mathbf{M}_{3}^{\delta} \alpha^{n}+\left(\beta^{n-\frac{1}{2}}\right)^{T} \mathbf{M}_{2}^{\nu} \beta^{n-\frac{1}{2}}=0
$$

This equation comes from the first order equations and can be shown to have unit magnitude eigenvalues for the amplification matrix just as the 0-form, 1-form formulation resulting in energy conservation. 


\subsection{Electrodynamic Wave Equation}

\subsubsection{Numerical stability}

The stability condition for the electrodynamic natural wave equation has the same

form as (5.13) with different eigenvalues $\kappa_{1}^{\nabla \times}$. In this case the matrix $\mathbf{B}$ is equal to (5.38) and the eigenvalues $\kappa_{1}^{\nabla \times}$ for the eigenvalue equation (5.39).

$$
\begin{gathered}
\mathbf{B}=2 \mathbf{I}-(\Delta t)^{2} \mathbf{M}_{1}^{-1} \mathbf{K}_{1} \\
\mathbf{M}_{1} \mathbf{x}=-\kappa_{1}^{\nabla \times} \mathbf{K}_{1} \mathbf{x}
\end{gathered}
$$

The stability condition is given in $(5.40)$

$$
\Delta t \leq \frac{2}{\sqrt{\max \left(\kappa_{1}^{\nabla \times}\right)}}
$$

As in the scalar wave equation the second-order electrodynamic wave equation is only weakly stable. In this case stability cannot be improved by eliminating the nullspace of the operator. The space dimensions for the natural Curl-Curl operator are shown in Table 5.5. The number of degenerate eigenvalues corresponds to the number of independent scalar functions which is the number of nodes minus 1 . The gradients of these scalar functions form the null-space of the operator and the amplification matrix. The equation admits solutions of the type $t \nabla \phi$ which is the non-physical solution creating the linear growth. If the growth in error is on the order of machine precision, then in practice the error will not be significant until millions of timesteps have elapsed. The spectrum, static, dynamic and invalid solutions for this operator are shown in Figure 5.3. The worst growth for this operator is linear growth for the same reason as in the scalar numerical stability Section 5.1. 
Table 5.5: Space dimensions for the natural Curl-Curl operator test equations on a hexahedral sphere grid.

\begin{tabular}{|c|c|c|c|c|}
\hline Operator & $\mathrm{n}$ & $2 \mathrm{n}$ & Distinct Eigenvalues & Deg. Eigenvalues \\
\hline \hline Curl-Curl & 202 & 404 & 326 & 78 \\
\hline
\end{tabular}
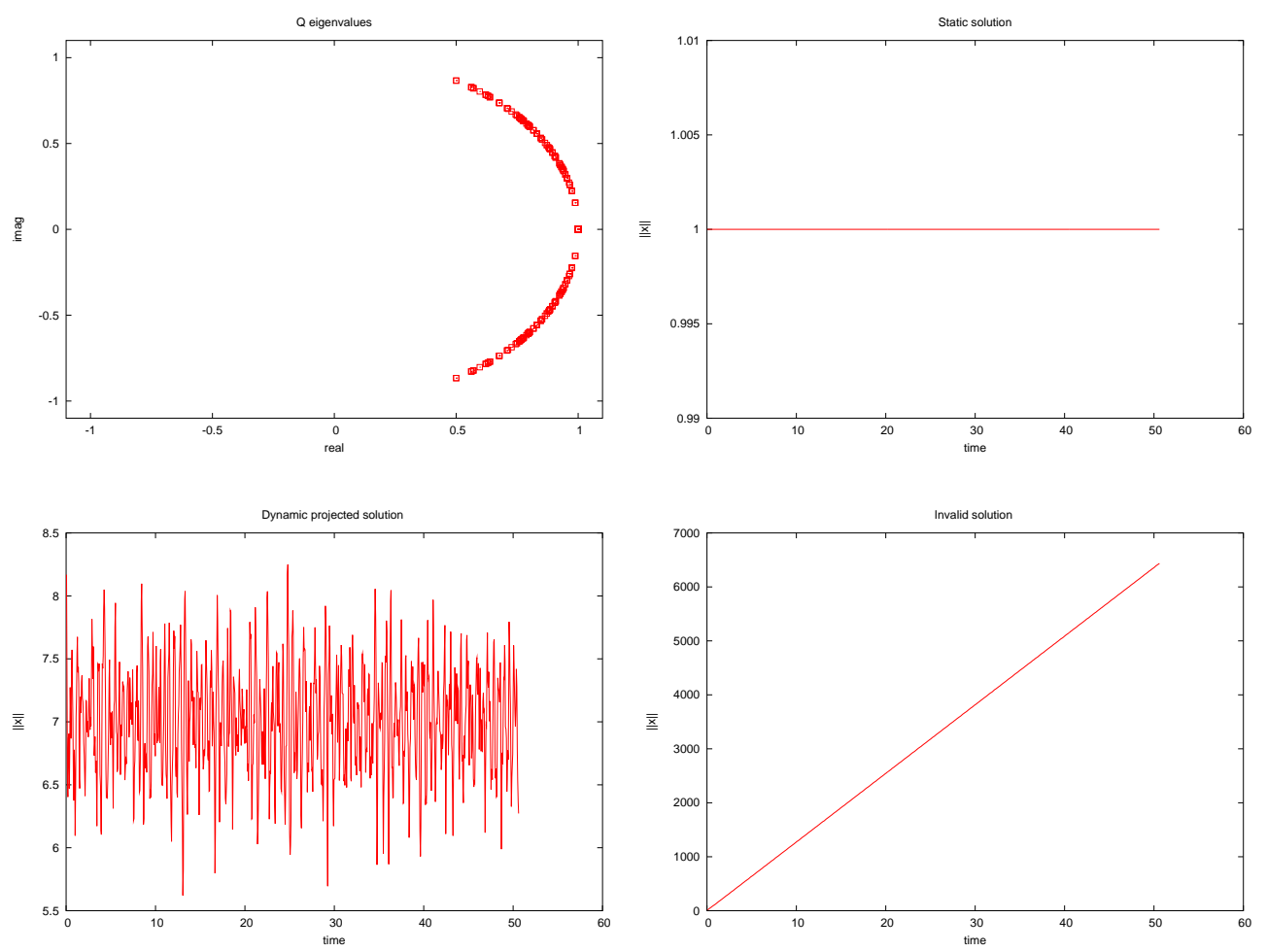

Figure 5.3: Second order natural Curl-Curl solutions 
The adjoint electrodynamic equation has a $\mathbf{B}$ matrix given by $\mathbf{B}=2 \mathbf{I}-(\Delta t)^{2} \mathbf{C M}_{1}^{-1} \mathbf{C}^{T} \mathbf{M}_{2}$.

The eigenvalues in this case are $\kappa_{2}^{\nabla \times}$ from the (5.41).

$$
\mathbf{x}=-\kappa_{2}^{\nabla \times} \mathbf{C M}_{1}^{-1} \mathbf{C}^{T} \mathbf{M}_{2} \mathbf{x}
$$

The stability condition is given in (5.42)

$$
\Delta t \leq \frac{2}{\sqrt{\max \left(\kappa_{2}^{\nabla \times}\right)}}
$$

The adjoint Curl-Curl operator is formed using the 2-forms. The gradient associated with this operator is of 3 -form scalar basis function. The null-space dimension is then the number of zones in the mesh as shown in Table 5.6. The spectrum, static, dynamic and invalid solutions are shown in Figure 5.4. The worst possible growth for the second-order adjoint electrodynamic wave equation is linear for the same reason as in Section 5.1.

Table 5.6: Space dimensions for the adjoint Curl-Curl operator test equations on a hexahedral sphere grid

\begin{tabular}{|c|c|c|c|c|}
\hline Operator & $\mathrm{n}$ & $2 \mathrm{n}$ & Distinct Eigenvalues & Deg. Eigenvalues \\
\hline \hline Curl-Curl & 180 & 360 & 304 & 56 \\
\hline
\end{tabular}

\subsubsection{Numerical dispersion}

The numerical dispersion for the second order electric wave equation is determined by assuming a solution of the form (5.43).

$$
\vec{E}(\vec{r}, t)=\tilde{E} e^{i(\vec{k} \cdot \vec{r}-\omega t)}
$$



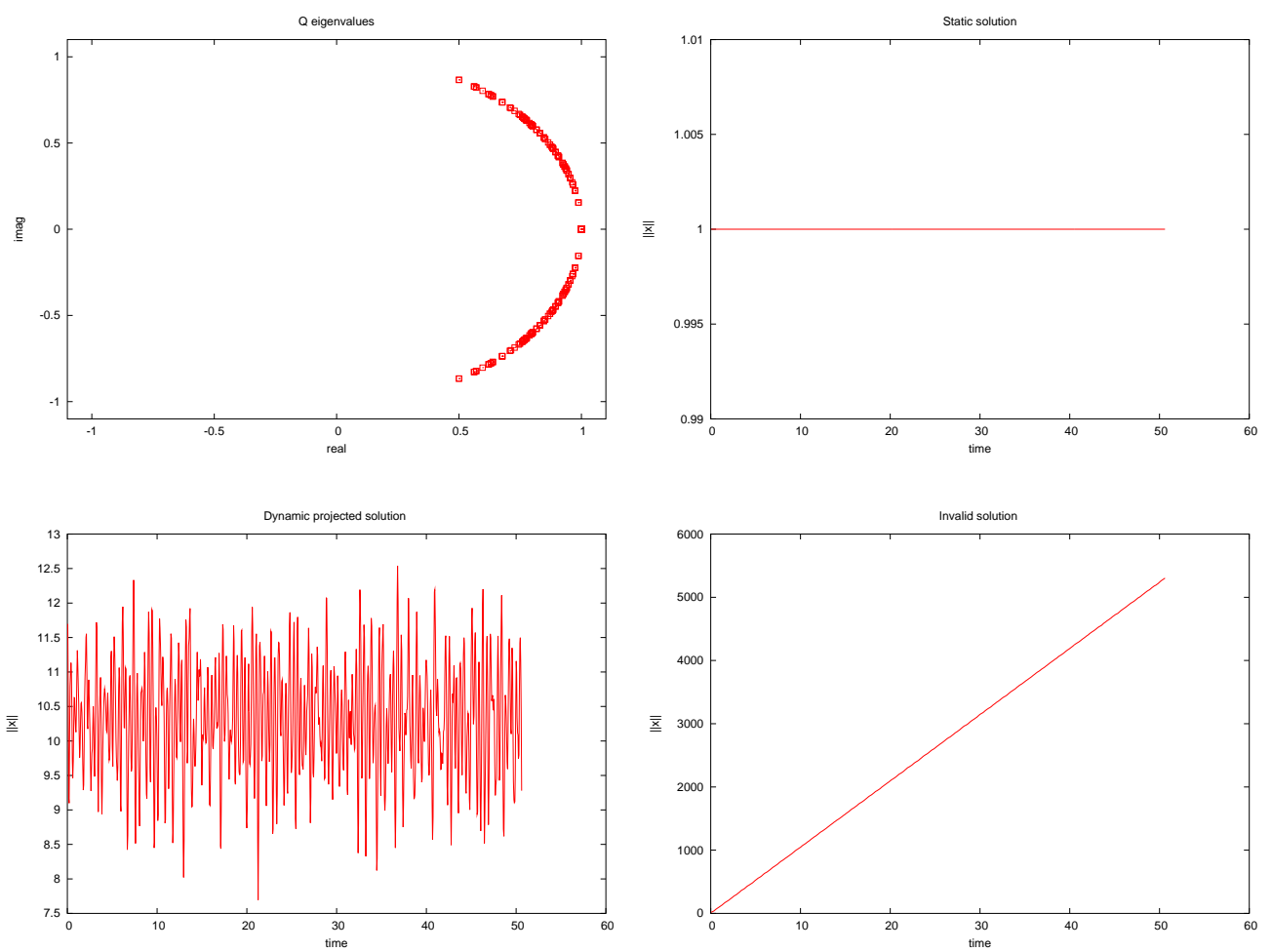

Figure 5.4: Second order adjoint Curl-Curl solutions 
For the plane wave solution to the electric field wave equation the dispersion relation is given by (5.44)

$$
\frac{\omega^{2}}{k^{2}}=c^{2}
$$

where $c$ is the speed of light. The 1-form natural form electric field wave equation dispersion analysis proceeds in the same manner as the 0 -form natural form scalar wave equation dispersion analysis. The vector valued nature of the electric field gives rise to three independent components $X, Y, Z$ for the plane wave solution. The edge degrees of freedom for the electric field on a single hexahedron element are given in Table 5.7. The numbering for these edge degrees of freedom can be found in Figure 3.11. The values of the quantities $\overrightarrow{\Delta x}_{(i, j)}$ correspond to the distance between the

Table 5.7: Edge Dispersion Degrees Of Freedom

\begin{tabular}{|c|c|c|c|c|c|}
\hline & Function & & Function & & Function \\
\hline$e_{1}$ & $X$ & $e_{5}$ & $Y$ & $e_{9}$ & $Z$ \\
\hline$e_{2}$ & $X e^{-(\vec{k} \cdot \overrightarrow{\Delta x}(1,2)-\omega \Delta t)}$ & $e_{6}$ & $Y e^{-(\vec{k} \cdot \overrightarrow{\Delta x}(5,6)-\omega \Delta t)}$ & $e_{10}$ & $Z e^{-(\vec{k} \cdot \overrightarrow{\Delta x}(9,10)-\omega \Delta t)}$ \\
\hline$e_{3}$ & 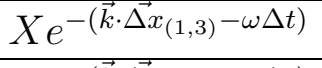 & $e_{7}$ & 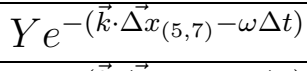 & $e_{11}$ & $Z e^{-(\vec{k} \cdot \overrightarrow{\Delta x}(9,11)-\omega \Delta t)}$ \\
\hline$e_{4}$ & $X e^{-(\vec{k} \cdot \overrightarrow{\Delta x}(1,4)-\omega \Delta t)}$ & $e_{8}$ & $Y e^{-(\vec{k} \cdot \overrightarrow{\Delta x}(5,8)-\omega \Delta t)}$ & $e_{12}$ & $Z e^{-(\vec{k} \cdot \overrightarrow{\Delta x}(9,12)-\omega \Delta t)}$ \\
\hline
\end{tabular}

midpoint of edge $i$ to the midpoint of edge $j$. Substituting the degrees of freedom into the second order electric field equation in an infinite, source and conductivity free region gives $(5.45)$.

$$
\frac{\partial^{2} \epsilon \vec{E}}{\partial t^{2}}=-\nabla \times \mu^{-1} \nabla \vec{E}
$$

The Galerkin form of this equation is given by (5.46).

$$
\mathbf{M}_{\mathbf{1}} \frac{\partial^{2} \vec{e}}{\partial t^{2}}=-\mathbf{K}_{\mathbf{1}} \vec{e}
$$


The matrices $\mathbf{M}_{\mathbf{1}}$ and $\mathbf{K}_{\mathbf{1}}$ correspond to the 1-form mass and stiffness matrices respectively and have been defined previously. Expanding the time derivative as in the scalar wave equation gives (5.47).

$$
\begin{array}{r}
\frac{\partial^{2} e_{i}}{\partial t^{2}} \approx \frac{e_{i}^{n+1}-2 e_{i}^{n}+e_{i}^{n-1}}{\Delta t^{2}}=\frac{\Psi e_{i}}{\Delta t^{2}} \\
\Psi=\frac{2}{\Delta t^{2}}(\cos (\omega \Delta t)-1)
\end{array}
$$

Entering the degrees of freedom in Table 5.7 and the discrete time derivative term from (5.47) yields a homogeneous system of equations with the three components of the plane wave $X, Y, Z$ as unknowns (5.49)

$$
\left(\Psi \mathbf{D m}_{1}+\nu \mathbf{D k}_{1}\right)(\alpha)=0
$$

The matrices $\mathbf{D m}_{1}$ and $\mathbf{D k}_{1}$ are non-linear functions of the wave vector $\vec{k}$ and the mass $\mathbf{M}_{\mathbf{1}}$ and stiffness $\mathbf{K}_{\mathbf{1}}$ matrices respectively. The value of $\nu$ is given for the speed of light $c$ is given by $\nu=c^{2} \frac{\Delta t^{2}}{\Delta h^{2}}$. The determinant of (5.49) gives three roots, one zero and two nonzero roots corresponding to the two polarizations. The roots are expanded around $\Delta h=0$ just as in the scalar wave equation dispersion analysis resulting in the same equation as in the scalar case (5.27). For the specific case of a uniform Cartesian grid $\vec{k}=a \hat{x}+b \hat{y}+c \hat{z}$ and Taylor expanding the root about $\Delta h=0$ gives the equation for the anisotropic term (5.86).

$$
R_{t e}=k^{2}\left(1+\frac{1}{12}(k \Delta h)^{2}+\mathcal{O}\left(\Delta h^{4}\right)\right)
$$

The root $R$ Taylor expanded around $\Delta t=0$ is the same as in the scalar case due to the identical leap frog time stepping method used in both simulations (5.51).

$$
R_{t e}=\omega^{2}\left(1-\frac{1}{12}(\omega \Delta t)^{2}+\mathcal{O}\left(\Delta t^{4}\right)\right)
$$


Combining the two Taylor expansions, the generalized dispersion relation for the 1form electric wave equation is shown to be second order accurate (5.52).

$$
\frac{\omega^{2}}{k^{2}}=c^{2} \frac{\left(1+\frac{1}{12}(k \Delta h)^{2}+\mathcal{O}\left(\Delta h^{4}\right)\right)}{\left(1-\frac{1}{12}(\omega \Delta t)^{2}+\mathcal{O}\left(\Delta t^{4}\right)\right)}
$$

\subsubsection{Magnetic charge conservation}

One of the charge conservation laws for electrodynamics is the conservation of magnetic charge (5.53).

$$
\nabla \cdot \frac{\partial \vec{B}}{\partial t}=0
$$

The inclusion relation between 1-forms and 2-forms can be used to rewrite Faraday's law resulting in conservation of (5.53). The curl of the 1-form basis functions $\overrightarrow{\mathcal{W}}$ can be written as a linear combination of the 2-form basis functions $\overrightarrow{\mathcal{F}}$ this is illustrated in Figure 5.5. The discrete electric field in a tetrahedron is given by (5.54).

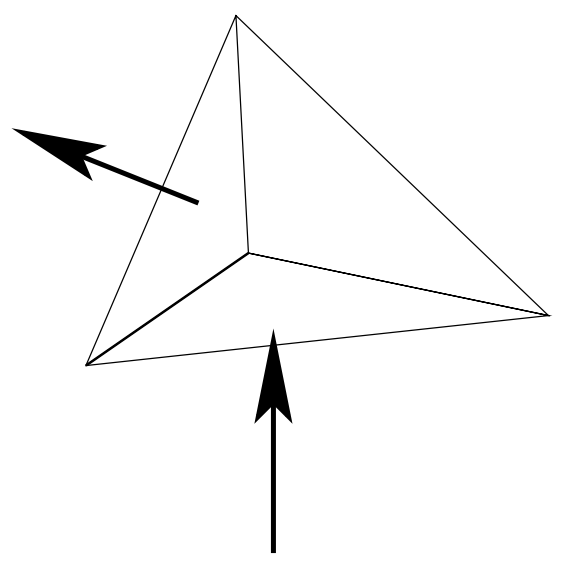

Figure 5.5: Discrete Curl for Highlighted Edge in a Tetrahedron

$$
\vec{E}=\sum_{i}^{6} e_{i} \vec{W}_{i}
$$


The time rate of change of the magnetic flux density becomes (5.55).

$$
\begin{array}{r}
\frac{\partial \vec{B}}{\partial t}=-\sum_{i}^{6} e_{i} \nabla \times \vec{W}_{i}=e_{1}\left(\vec{F}_{2}-\vec{F}_{1}\right)+e_{2}\left(\vec{F}_{1}-\vec{F}_{3}\right)+e_{3}\left(\vec{F}_{3}-\vec{F}_{2}\right) \\
+e_{4}\left(\vec{F}_{4}-\vec{F}_{1}\right)+e_{5}\left(\vec{F}_{2}-\vec{F}_{4}\right)+e_{6}\left(\vec{F}_{4}-\vec{F}_{3}\right) \\
=\vec{F}_{1}\left(e_{2}-e_{1}-e_{4}\right)+\vec{F}_{2}\left(e_{1}+e_{5}-e_{3}\right)+\vec{F}_{3}\left(e_{3}-e_{2}-e_{6}\right)+\vec{F}_{4}\left(e_{4}+e_{6}-e_{5}\right)
\end{array}
$$

Applying Gauss's law to equation (5.55) shows that the degrees of freedom will sum to zero (5.56).

$$
\nabla \cdot \frac{\partial \vec{B}}{\partial t}=0
$$

The magnetic charge constraint is satisfied exactly for the magnetic flux density expanded in 2-forms. The type of element does not make a difference in the analysis and therefore this result applies to any unstructured grid.

\subsubsection{Electric charge conservation}

The 1-form electric charge conservation is satisfied in the variational sense. This can be shown by looking at the second order electric field equation in the frequency domain (5.57) with boundary condition $\vec{E} \times \hat{n}=0$ on $\Gamma$.

$$
\nabla \times \mu^{-1} \nabla \times \vec{E}-\omega^{2} \epsilon \vec{E}=0
$$

This equation admits two solution types, irrotational and solenoidal fields. The irrotational fields are characterized by an electric field that can be written as the gradient of the scalar potential $\phi$ (5.58) giving $\omega=0$ for (5.57).

$$
\vec{E}_{i r}=-\nabla \phi
$$


For eigenvalues $\omega \neq 0$ the electric field is solenoidal which can be seen by taking the divergence of (5.57) resulting in (5.59).

$$
\nabla \cdot \epsilon \vec{E}_{\text {sol }}=0
$$

If the initial electric field and sources are divergence free then (5.59) will be true for all time if and only if Curl-Curl operator is divergence free. The derivation of the divergence free nature of the Curl-Curl operator follows. The permittivity may not be continuous so it is best to look at (5.59) in variational form. Multiplying (5.59) by the potential $\phi$ results in the variational form (5.60).

$$
\int_{\Omega} \epsilon \vec{E}_{s o l} \cdot \nabla \phi
$$

The discrete version of (5.57) can be constructed by using the matrices generated in Chapter 3 resulting in the discrete second-order electric field equation in the frequency domain (5.61).

$$
\mathbf{K}_{1}^{\mu^{-1}} \vec{e}-\omega^{2} \mathbf{M}_{1}^{\epsilon} \vec{e}=0
$$

The discrete 1-form space $\mathcal{W}^{h} \subset \mathcal{H}_{0}($ curl $)$ can written as the sum of the irrotational fields space and the solenoidal fields space (5.62).

$$
\mathcal{W}^{h}=\mathcal{W}_{i r}^{h}+\mathcal{W}_{\text {sol }}^{h}
$$

This sum is referred to as the discrete Helmholtz decomposition of $\mathcal{W}^{h}$. In this decomposition the irrotational subspace is orthogonal to the solenoidal subspace $\mathcal{W}_{i r}^{h}=\left(\mathcal{W}_{\text {sol }}^{h}\right)^{\perp}$. The irrotational and solenoidal subspaces are defined by $(5.63)$ and (5.64) respectively.

$$
\mathcal{W}_{i r}^{h}=\left\{\vec{v} \in \mathcal{W}^{h} ; \nabla \times \vec{v}=0\right\}
$$




$$
\mathcal{W}_{\text {sol }}^{h}=\left\{\vec{v} \in \mathcal{W}^{h} ; \nabla \cdot \vec{v}=0\right\}
$$

Using the discrete gradient operator listed in (3.63), the discrete version of (5.58) can be constructed resulting in (5.65).

$$
\vec{e}_{i r}=G \vec{f} \quad ; \vec{e}_{i r} \in V ; \vec{f} \in F
$$

where $F=\left\{\vec{f} \in R^{k} ; \tilde{\phi} \in \mathcal{N}^{h}, \tilde{\phi} \Rightarrow \vec{f}\right\}, \mathcal{N}^{h} \subset \mathcal{H}_{0}($ grad $)$ and $V=\left\{\vec{e}_{\text {ir }}\right.$ in $R^{n}, \tilde{E}_{i r} \in$ $\left.\mathcal{W}_{i r}^{h}, \tilde{E}_{i r} \Rightarrow \vec{e}_{i r}\right\}$. In these spaces the notation $\tilde{\phi} \Rightarrow \vec{f}$ denotes the construction of $\vec{f}$ given $\tilde{\phi}$. The vectors $\vec{e}_{i r}$ form the null space of the stiffness matrix $\mathbf{K}_{1}^{\mu^{-1}}$ (5.66). The tildes on the variables indicate they are the discrete versions of the continuous variables.

$$
\mathbf{K}_{1}^{\mu^{-1}} \mathbf{G} \vec{f}=0 \forall \vec{f}
$$

This shows that there are $k$ solutions of (5.61) with $\omega=0$, giving the static solutions with nonzero divergence.

Choosing a solenoidal field vector $\vec{e}_{\text {sol }}$ from the space $U=\left\{\vec{e}_{\text {sol }} \in R^{n} ; \tilde{E}_{\text {sol }} \in\right.$ $\left.\mathcal{W}_{\text {sol }}^{h}, \tilde{E}_{\text {sol }} \Rightarrow \vec{e}_{\text {sol }}\right\}$ defines the Rangespace of (5.61) giving (5.67).

$$
\mathbf{K}_{1}^{\mu^{-1}} \vec{e}_{s o l}=\omega^{2} \mathbf{M}_{1}^{\epsilon} \vec{e}_{s o l}
$$

Multiplying this with an arbitrary irrotational $\vec{e}_{i r} \in V$ and using (5.66) gives (5.68).

$$
\vec{e}_{i r}^{T} \mathbf{K}_{1}^{\mu^{-1}} \vec{e}_{\text {sol }}=\vec{f}^{T} \mathbf{K}_{1}^{\mu^{-1}} \mathbf{G}^{T} \vec{e}_{\text {sol }}=\omega^{2} \vec{f}^{T} \mathbf{G}^{T} \mathbf{M}_{1}^{\epsilon} \vec{e}_{\text {sol }}=0
$$

The discrete solenoidal solutions are orthogonal to the discrete irrotational solutions through the inner product $\vec{y}^{T} \mathbf{M}_{1}^{\epsilon} \vec{x}$.

The conservation of electric charge is derived from (5.68) where (5.69) is the discrete version of $(5.60)$.

$$
\vec{f}^{T} \mathbf{G}^{T} \mathbf{M}_{1}^{\epsilon} \vec{e}_{\text {sol }}=0
$$


The discrete solenoidal solutions $\vec{e}_{\text {sol }}$ satisfy the discrete divergence free condition in the variational sense resulting in the conservation of electric charge.

\subsubsection{Energy conservation}

The energy for the electrodynamic wave equation is given in (2.30). To show the conservation of energy the integrodifferential form is used (5.70)

$$
\oint_{\Gamma}(\vec{E} \times \vec{H}) \cdot \hat{n} d \Gamma+\int_{\Omega} \mu^{-1} \vec{B} \cdot \frac{\partial \vec{B}}{\partial t}+\int_{\Omega} \epsilon \vec{E} \cdot \frac{\partial \vec{E}}{\partial t}=0
$$

This equation represents the Poynting theorem for a domain free of conductivity. The first term represents the energy flowing into the domain while the second and third terms represent the time rate of change of the energy in the magnetic and electric fields respectively. Using the 1-form and 2-form mass matrices $\mathbf{M}_{1}$ and $\mathbf{M}_{2}$ for the electric field term and magnetic flux density term respectively the discrete version of (5.70) can be derived and is shown in (5.71). In this equation the energy flowing into the domain is set to be zero.

$$
e^{T} \mathbf{M}_{1}^{\epsilon} \frac{\partial e}{\partial t}+b^{T} \mathbf{M}_{2}^{\mu^{-1}} \frac{\partial b}{\partial t}=0
$$

Using the leap frog integration scheme for the single time derivative equations (5.2) in (5.71) the energy equation (5.72) can be shown to conserve energy in a time averaged sense.

$$
\left(e^{n+1}\right)^{T} \mathbf{M}_{1}^{\epsilon} e^{n+1}+\left(b^{n+\frac{1}{2}}\right)^{T} \mathbf{M}_{2}^{\mu^{-1}} b^{n+\frac{1}{2}}+\left(e^{n}\right)^{T} \mathbf{M}_{1}^{\epsilon} e^{n}+\left(b^{n-\frac{1}{2}}\right)^{T} \mathbf{M}_{2}^{\mu^{-1}} b^{n-\frac{1}{2}}=0
$$

This result is derived through an analysis of the first order equations as done with the second order equations in the stability section. The result of the first order stability 
analysis is the same as the second order result (5.40). The amplification matrix for the first order equations also has eigenvalues with unit magnitude. Using this fact the energy equation is constant in time and therefore conserves energy.

\subsection{Linear Acoustic Vector Wave Equation}

\subsubsection{Numerical stability}

The stability conditions for the acoustic wave equations have the same form as the previous sections. The difference is only in the eigenvalues of the wave equations.

The natural acoustic have equation has eigenvalues $\kappa_{2}^{\nabla \cdot}$ from (5.73) and the matrix B given by (5.74).

$$
\begin{gathered}
\mathbf{M}_{2} \mathbf{x}=-\kappa_{2}^{\nabla \cdot} \mathbf{K}_{2} \mathbf{x} \\
\mathbf{B}=2 \mathbf{I}-(\Delta t)^{2} \mathbf{M}_{2}^{-1} \mathbf{K}_{2}
\end{gathered}
$$

The stability condition is given in (5.75)

$$
\Delta t \leq \frac{2}{\sqrt{\max \left(\kappa_{2}^{\nabla \cdot}\right)}}
$$

The natural Grad-Div operator also has a large null-space that cannot be eliminated. This leads to a number of degenerate eigenvalues of the amplification matrix. In this case the degenerate eigenvalues correspond to vector functions with nonzero curls. The equation admits a solution of the type $t \nabla \times \vec{\Psi}$. The non-physical solution in this case is $t \nabla \times \vec{\Psi}$. The dimension of the null space results from the internal 1-form degrees of freedom minus the gradients of scalar functions plus the boundary face degrees of freedom. The dimensions for the amplification matrix are shown in 
Table 5.8. The eigenvalue spectrum, static, dynamic and invalid solutions are shown in Figure 5.6. The worst possible growth for this operator are the same as in all of the other operators, linear growth, for the same reason as in Section 5.1.

Table 5.8: Space dimensions for the natural Grad-Div operator test equations on a hexahedral sphere grid.

\begin{tabular}{|c|c|c|c|c|}
\hline Operator & $\mathrm{n}$ & $2 \mathrm{n}$ & Distinct Eigenvalues & Deg. Eigenvalues \\
\hline \hline Grad-Div & 180 & 360 & 236 & 124 \\
\hline
\end{tabular}
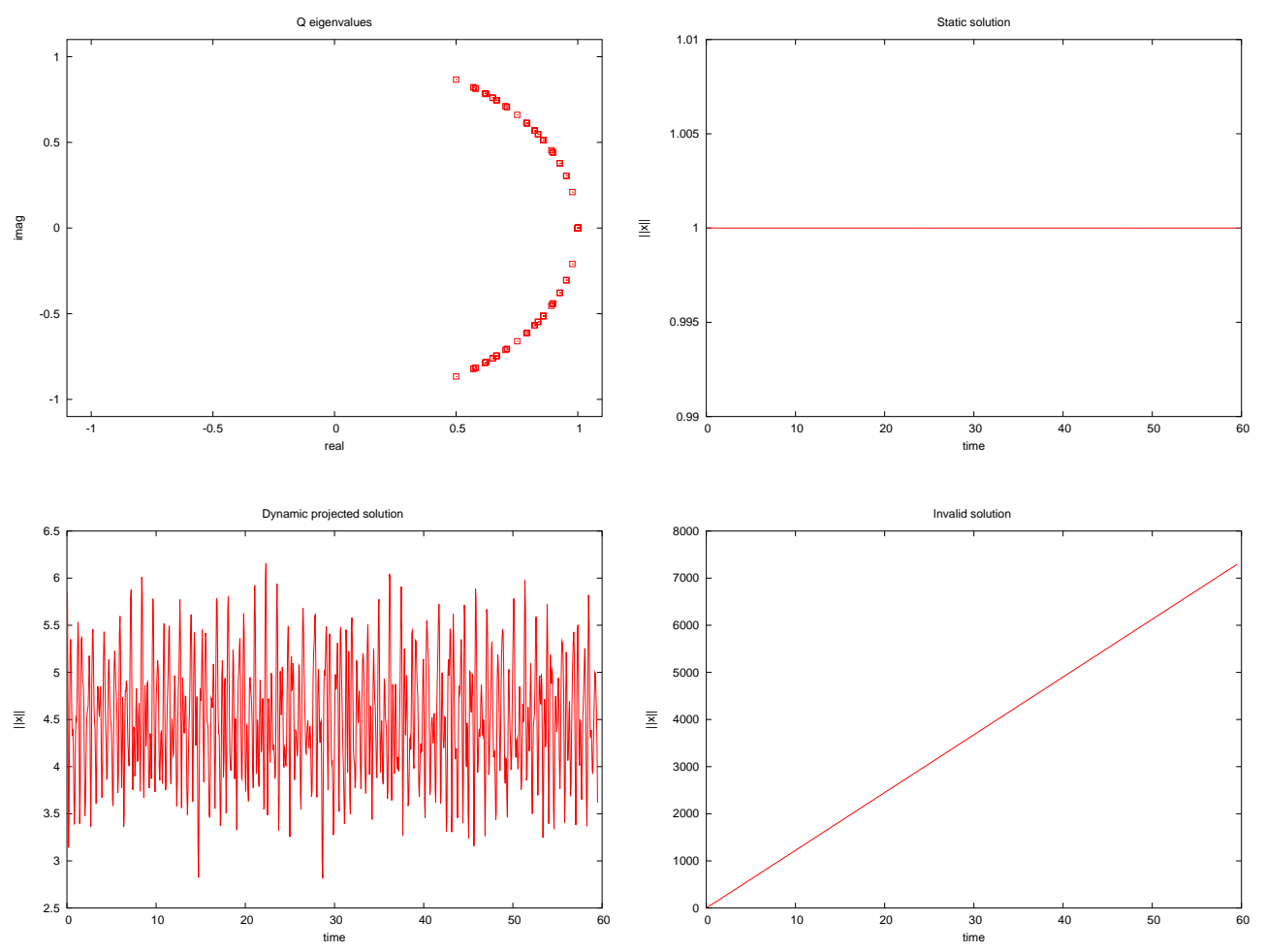

Figure 5.6: Second order natural Grad-Div solutions

The adjoint acoustic wave equation has eigenvalues $\kappa_{1}^{\nabla \cdot}$ from (5.76) and the matrix B given by (5.77)

$$
\begin{gathered}
\mathbf{x}=-\kappa_{1}^{\nabla \cdot} \mathbf{G M}_{0}^{-1} \mathbf{G}^{T} \mathbf{M}_{1} \mathbf{x} \\
\mathbf{B}=2 \mathbf{I}-(\Delta t)^{2} \mathbf{G M}_{0}^{-1} \mathbf{G}^{T} \mathbf{M}_{1}
\end{gathered}
$$


The stability condition is given in (5.78)

$$
\Delta t \leq \frac{2}{\sqrt{\max \left(\kappa_{1}^{\nabla \cdot}\right)}}
$$

The space dimensions shown in Table 5.9 show the same number of degenerate eigenvalues. This is due to the dual nature of the curl operator. The curl of a 1-form is a 2-form and vice versa. The eigenvalue spectrum for the adjoint Grad-Div operator, static, dynamic and invalid solutions are shown in Figure 5.7. The worst growth for this operator is linear growth for the same reason as in Section 5.1.

Table 5.9: Space dimensions for the adjoint Grad-Div operator test equations on a hexahedral sphere grid

\begin{tabular}{|c|c|c|c|c|}
\hline Operator & $\mathrm{n}$ & 2n & Distinct Eigenvalues & Deg. Eigenvalues \\
\hline \hline Grad-Div & 202 & 404 & 280 & 124 \\
\hline
\end{tabular}

\subsubsection{Numerical dispersion}

The numerical dispersion for the second order velocity wave equation is determined by assuming a solution of the form (5.79).

$$
\vec{v}(\vec{r}, t)=\tilde{v} e^{i(\vec{k} \cdot \vec{r}-\omega t)}
$$

For the plane wave solution to the velocity field wave equation the dispersion relation is given by (5.80)

$$
\frac{\omega^{2}}{k^{2}}=c_{l}^{2}
$$

where $c_{l}$ is the speed of sound as in the scalar wave equation. The 2-form natural form electric field wave equation dispersion analysis proceeds in the same manner as the 1-form natural electric field wave equation dispersion analysis. The vector valued 

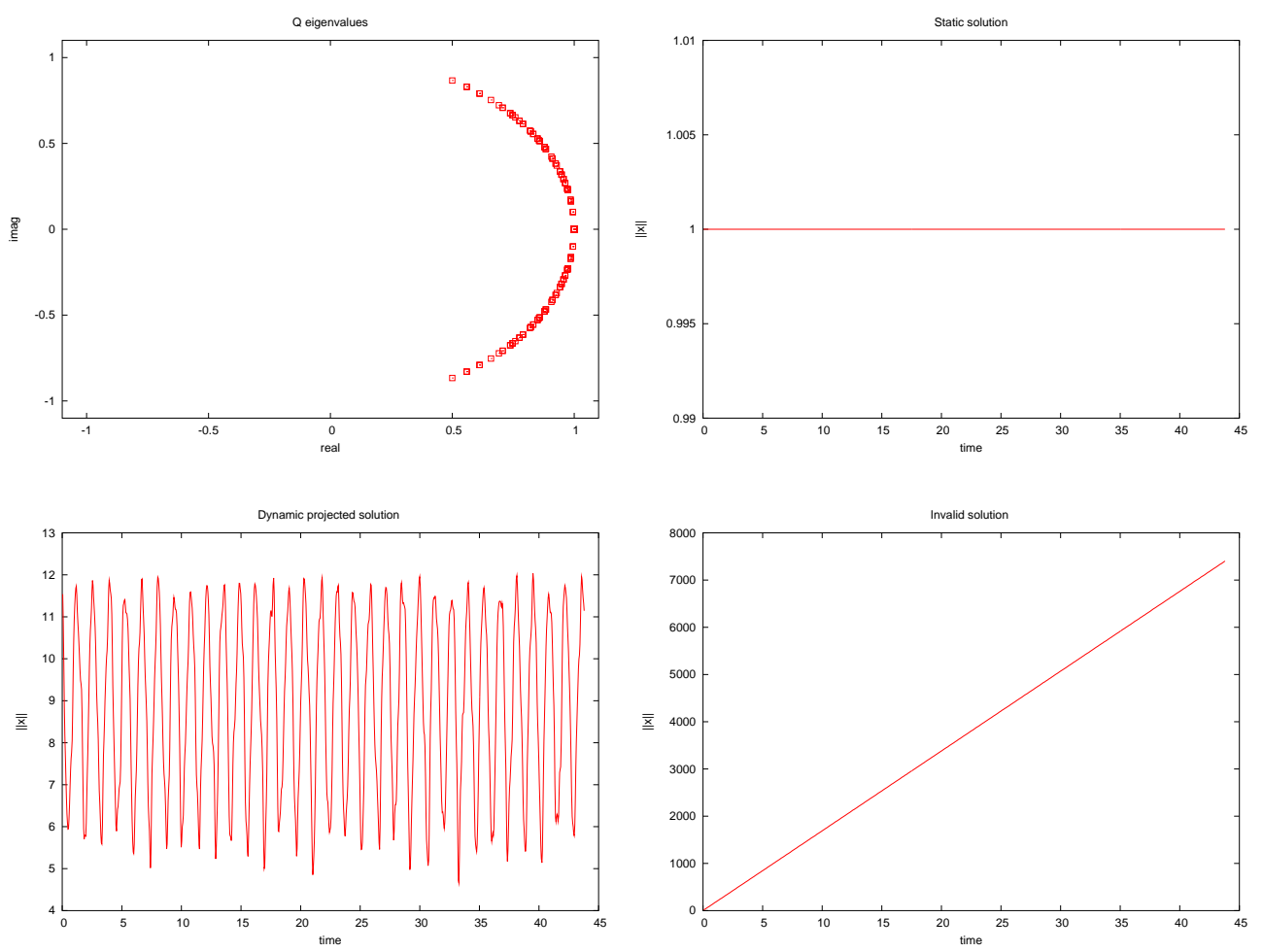

Figure 5.7: Second order adjoint Grad-Div solutions 
nature of the electric field again gives rise to three independent components $X, Y, Z$ for the plane wave solution. The face degrees of freedom for the velocity field on a single hexahedron element are given in Table 5.10. The numbering for these face degrees of freedom can be found in Figure 3.11.

Table 5.10: Face Dispersion Degrees Of Freedom

\begin{tabular}{||c|c||c|c||c|c||}
\hline & Function & & Function & & Function \\
\hline \hline$f_{1}$ & $X$ & $f_{3}$ & $Y$ & $f_{5}$ & $Z$ \\
\hline$f_{2}$ & $\left.X e^{-(\vec{k} \cdot \overrightarrow{\Delta x}(1,2)}-\omega \Delta t\right)$ & $f_{4}$ & $\left.Y e^{-(\vec{k} \cdot \overrightarrow{\Delta x}(3,4)}-\omega \Delta t\right)$ & $f_{6}$ & $Z e^{-(\vec{k} \cdot \overrightarrow{\Delta x}(5,6)-\omega \Delta t)}$ \\
\hline
\end{tabular}

The values of the quantities $\overrightarrow{\Delta x_{(i, j)}}$ correspond to the distance between the face centroid of face $i$ to the face centroid of face $j$. Substituting the degrees of freedom into the second order velocity field equation in an infinite, source free region gives (5.81).

$$
\frac{\partial^{2} \vec{v}}{\partial t^{2}}=c_{l}^{2} \nabla \nabla \cdot \vec{v}
$$

The Galerkin form of this equation is given by (5.82).

$$
\mathbf{M}_{\mathbf{2}} \frac{\partial^{2} \vec{\alpha}}{\partial t^{2}}=-\mathbf{K}_{\mathbf{2}} \vec{\alpha}
$$

The matrices $\mathbf{M}_{\mathbf{2}}$ and $\mathbf{K}_{\mathbf{2}}$ correspond to the 2-form mass and stiffness matrices respectively and have been defined previously. Expanding the time derivative as in the scalar and electric field wave equations gives (5.83).

$$
\begin{array}{r}
\frac{\partial^{2} \alpha_{i}}{\partial t^{2}} \approx \frac{\alpha_{i}^{n+1}-2 \alpha_{i}^{n}+\alpha_{i}^{n-1}}{\Delta t^{2}}=\frac{\Psi \alpha_{i}}{\Delta t^{2}} \\
\Psi=\frac{2}{\Delta t^{2}}(\cos (\omega \Delta t)-1)
\end{array}
$$

Entering the degrees of freedom in Table 5.10 and the discrete time derivative term from (5.83) yields a homogeneous system of equations with the three components of 
the plane wave $X, Y, Z$ as unknowns (5.85)

$$
\left(\frac{2 \Psi}{\Delta t^{2}} \mathbf{D m}_{2}+\nu \mathbf{D k}_{2}\right)=0
$$

The matrices $\mathbf{D m}_{2}$ and $\mathbf{D k}_{2}$ are non-linear functions of the wave vector $\vec{k}$ and the mass $\mathbf{M}_{2}$ and stiffness $\mathbf{K}_{1}$ matrices respectively. The value of $\nu$ is the same as in the scalar dispersion analysis $\nu=c_{l}^{2} \frac{\Delta t^{2}}{\Delta h^{2}}$. The roots are expanded around $\Delta h=0$ just as in the scalar wave equation dispersion analysis resulting in the same equation as in the scalar case (5.27). For the specific case of a uniform Cartesian grid $\vec{k}=a \hat{x}+b \hat{y}+c \hat{z}$ and Taylor expanding the root about $\Delta h=0$ gives the equation for the anisotropic term (5.86).

$$
R_{t e}=k^{2}\left(1+\frac{1}{12}(k \Delta h)^{2}+\mathcal{O}\left(\Delta h^{4}\right)\right)
$$

The root $R$ Taylor expanded around $\Delta t=0$ is the same as in the scalar case due to the identical leap frog time stepping method used in both simulations (5.87).

$$
R_{t e}=\omega^{2}\left(1-\frac{1}{12}(\omega \Delta t)^{2}+\mathcal{O}\left(\Delta t^{4}\right)\right)
$$

Combining the two Taylor expansions, the generalized dispersion relation for the 2form electric wave equation is shown to be second order accurate (5.88).

$$
\frac{\omega^{2}}{k^{2}}=c_{l}^{2} \frac{\left(1+\frac{1}{12}(k \Delta h)^{2}+\mathcal{O}\left(\Delta h^{4}\right)\right)}{\left(1-\frac{1}{12}(\omega \Delta t)^{2}+\mathcal{O}\left(\Delta t^{4}\right)\right)}
$$

\subsubsection{Vorticity conservation 1-forms}

The vector acoustic wave equations are solved with vorticity set to zero (5.89).

$$
\nabla \times \vec{v}=0
$$


This constraint is automatically maintained for the natural 2-form formulation and the adjoint 1-form formulation.

Beginning with the 1-form formulation it will be shown that the vorticity constraint is satisfied exactly. The vorticity must be satisfied in space and time, this results in the vorticity equation becoming (5.90).

$$
\nabla \times \frac{\partial \vec{v}}{\partial t}=0
$$

The inclusion relation between 1 -forms and 0 -forms can be used to rewrite the first order velocity equation (2.3). The gradients of the nodal basis functions $\mathcal{N}$ can be written as a linear combination of the edge basis functions $\mathcal{W}$, as illustrated in Figure 5.8. The pressure in a tetrahedron is given by (5.91).

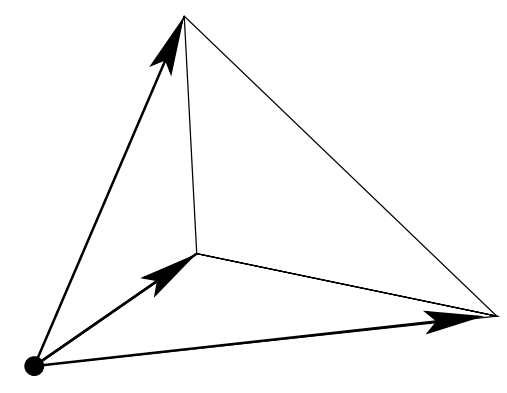

Figure 5.8: Discrete Gradient for Highlighted Node in a Tetrahedron

$$
P=\sum_{i}^{4} \beta_{i} N_{i}
$$

The time rate of change of the velocity with $\rho_{0}=1$, which is constant throughout the element, becomes (5.92).

$$
\begin{array}{r}
\frac{\partial \vec{v}}{\partial t}=-\sum_{i}^{4} \beta_{i} \nabla N_{i}=\beta_{1}\left(\vec{W}_{1}+\vec{W}_{2}+\vec{W}_{3}\right)+\beta_{2}\left(\vec{W}_{4}+\vec{W}_{5}-\vec{W}_{1}\right)+ \\
\beta_{3}\left(\vec{W}_{6}-\vec{W}_{2}-\vec{W}_{4}\right)+\beta_{4}\left(-\vec{W}_{3}-\vec{W}_{5}-\vec{W}_{6}\right)
\end{array}
$$




$$
\begin{array}{r}
=\vec{W}_{1}\left(\beta_{1}-\beta_{2}\right)+\vec{W}_{2}\left(\beta_{1}-\beta_{3}\right)+\vec{W}_{3}\left(\beta_{1}-\beta_{4}\right)+ \\
\vec{W}_{4}\left(\beta_{2}-\beta_{3}\right)+\vec{W}_{5}\left(\beta_{2}-\beta_{4}\right)+\vec{W}_{6}\left(\beta_{3}-\beta_{4}\right)
\end{array}
$$

Applying Stoke's theorem to equation (5.92) shows that the degrees of freedom will sum to zero (5.93).

$$
\begin{array}{r}
\nabla \times \frac{\partial \vec{v}}{\partial t}=\left(\beta_{1}-\beta_{2}\right)\{0,-2,2\}+\left(\beta_{1}-\beta_{3}\right)\{2,0,-2\}+\left(\beta_{1}-\beta_{4}\right)\{-2,2,0\}+ \\
\left(\beta_{2}-\beta_{3}\right)\{0,0,2\}+\left(\beta_{2}-\beta_{4}\right)\{0,-2,0\}+\left(\beta_{3}-\beta_{4}\right)\{2,0,0\}=0
\end{array}
$$

The vorticity constraint is satisfied exactly for the 1 -form velocity wave equation. The type of element does not make a difference in the analysis and therefore this result applies to any unstructured grid.

\subsubsection{Vorticity conservation 2-forms}

The 2-form vorticity constraint is satisfied in the variational sense similar to the 1 form electric charge conservation. Beginning with the second-order velocity equation in the frequency domain (5.94) with boundary condition $\vec{v} \cdot \hat{n}=0$ on $\Gamma$, two types of solutions are admitted, solenoidal and irrotational.

$$
\nabla c_{l}^{2} \nabla \cdot \vec{v}+\omega^{2} \vec{v}=0
$$

For $\omega=0$ the solution is a solenoidal field which can be characterized by the curl of a scalar potential $\vec{A}$ shown in (5.95).

$$
\nabla \cdot \vec{v}=0 ; \vec{v}=\nabla \times \vec{A}
$$

The solutions for $\omega \neq 0$ are irrotational fields (5.96) derived by taking the curl of (5.94).

$$
\nabla \times \vec{v}=0
$$


If the velocity and sources are curl free initially then (5.96) will be true for all time if and only if the Grad-Div operator is curl free. The derivation of the curl free nature of the Grad-Div operator follows. Looking at the irrotational term in the variational sense gives (5.97) due to potential discontinuities in the sound speed $c_{l}$.

$$
\int_{\Omega} \vec{v} \cdot \nabla \times \vec{A}=0
$$

A discrete version of (5.94) is constructed with the use of the matrices defined in Chapter 3 giving the discrete second-order velocity wave equation in the frequency domain (5.98).

$$
\mathbf{K}_{2}^{c_{l}^{2}} \vec{v}-\omega^{2} \mathbf{M}_{2} \vec{v}=0
$$

The discrete 2-form space $\mathcal{F}^{h} \subset \mathcal{H}_{0}($ div $)$ can be decomposed into irrotational and solenoidal subspaces (5.99).

$$
\mathcal{F}^{h}=\mathcal{F}_{i r}^{h}+\mathcal{F}_{\text {sol }}^{h}
$$

This is the Helmholtz decomposition of $\mathcal{F}^{h}$ where $\mathcal{F}_{\text {ir }}^{h}=\left(\mathcal{F}_{\text {sol }}^{h}\right)^{\perp}$ which are defined in (5.100) and (5.101).

$$
\begin{aligned}
& \mathcal{F}_{\text {ir }}^{h}=\left\{\vec{v} \in \mathcal{F}^{h} ; \nabla \times \vec{v}=0\right\} \\
& \mathcal{F}_{\text {sol }}^{h}=\left\{\vec{v} \in \mathcal{F}^{h} ; \nabla \cdot \vec{v}=0\right\}
\end{aligned}
$$

Using a differential forms argument, the natural description of the vector potential $\vec{A}$ is a 1-form variable. This leads to the discrete version of (5.95) giving (5.102).

$$
\vec{v}_{\text {sol }}=\mathbf{C} \vec{a} \quad ; \vec{v}_{\text {sol }} \in V \text { and } \vec{a} \in A
$$

where $V=\left\{\vec{v}_{\text {sol }} \in R^{n}, \tilde{v} \in \mathcal{F}_{\text {sol }}^{h}, \tilde{v} \Rightarrow \vec{v}_{\text {sol }}\right\}$ and $A=\left\{\vec{a} \in R^{k}, \tilde{A} \in \mathcal{W}^{h}, \tilde{A} \Rightarrow \vec{a}\right\}$ and $\Rightarrow$ is defined as in Section 5.2.4. 
The solenoidal velocity vectors $\vec{v}_{s o l} \in V$ form the null space of the stiffness matrix $\mathbf{K}_{2}^{c_{2}^{2}}$ shown in (5.103).

$$
\mathbf{K}_{2}^{c_{l}^{2}} \mathbf{C} \vec{a}=0 \forall \vec{a}
$$

Choosing an irrotational velocity $\vec{v}_{i r}$ from the space $U=\left\{\vec{v}_{i r} \in R^{n}, \tilde{v} \in \mathcal{W}_{i r}^{h}, \tilde{v} \Rightarrow\right.$ $\left.\vec{v}_{i r}\right\}$ and substituting these solutions into (5.98) results in the equation (5.104).

$$
\mathbf{K}_{2}^{c_{l}^{2}} \vec{v}_{i r}=\omega^{2} \mathbf{M}_{2} \vec{v}_{i r}
$$

Multiplying (5.104) by an arbitrary solenoidal velocity vector and using (5.103) results in (5.105).

$$
\vec{v}_{\text {sol }}^{T} \mathbf{K}_{2}^{c_{c}^{2}} \vec{v}_{i r}=\vec{a}^{T} \mathbf{C K}_{2}^{c_{l}^{2}} \vec{v}_{i r}=\omega^{2} \vec{a}^{T} \mathbf{C M}_{2} \vec{v}_{i r}=0
$$

The discrete solenoidal solutions are orthogonal to the discrete irrotational solutions through the inner product $\vec{y}^{T} \mathbf{M}_{2} \vec{x}$.

The conservation of vorticity is derived from (5.105) where (5.106) is the discrete version of (5.97).

$$
\vec{a}^{T} \mathbf{C}^{T} \mathbf{M}_{2} \vec{v}_{i r}=0
$$

The discrete irrotational solutions $\vec{V}_{i r}$ satisfy the discrete curl free condition in the variational sense resulting in the conservation of vorticity.

\subsubsection{Energy conservation}

The energy for the vector acoustic wave equation is given in (2.22). The vector acoustic wave equation has the same form for the first order equations as the scalar wave equation above. The energy conservation is therefore the same as shown in 
the scalar wave equation section above. Both the 0 -form-1-form and 2-form-3-form formulations conserve energy in a time averaged sense.

\subsection{Linear Elastic Wave Equation}

\subsubsection{Numerical stability}

The stability condition for the linear elastic wave equation combines the natural and adjoint operators for each form. The stability condition is still calculated in the same manner. The $\mathbf{B}$ matrix for the 1-form linear elastic equation is given by (5.107).

$$
\mathbf{B}=2 \mathbf{I}-(\Delta t)^{2}\left[\mathbf{K}_{1}+\mathbf{G M}_{0}^{-1} \mathbf{G}^{T} \mathbf{M}_{1}\right]
$$

The stability condition is given in (5.108)

$$
\Delta t \leq \frac{2}{\sqrt{\left[\max \left(\kappa_{1}^{\nabla \times}\right)+\max \left(\kappa_{1}^{\nabla \cdot}\right)\right]}}
$$

The $\mathbf{B}$ matrix for the $2-$ form linear elastic equation is given by (5.109).

$$
\mathbf{B}=2 \mathbf{I}-(\Delta t)^{2}\left[\mathbf{K}_{2}+\mathbf{C M}_{1}^{-1} \mathbf{C}^{T} \mathbf{M}_{2}\right]
$$

The stability condition is given in (5.110)

$$
\Delta t \leq \frac{2}{\sqrt{\left[\max \left(\kappa_{2}^{\nabla \times}\right)+\max \left(\kappa_{2}^{\nabla \cdot}\right)\right]}}
$$

\subsubsection{Numerical dispersion}

The numerical dispersion for the linear elastic wave equations can be determined by combining the three terms of the vector Laplacian operator shown for the 1-form in Figure 4.10 and for the 2-form in Figure 4.13. It is apparent from these diagrams 
that the three terms of the Laplacian for both $p$-forms are decoupled. The operators can therefore be combined into one larger stencil that represents the full Laplacian.

The dispersion is determined by evaluating each operator comprising the vector Laplacian operator separately. The coefficients of the stencil for each operator are combined in the plane wave solution (5.111) and evaluated at the edge or face centers.

$$
\vec{u}=\tilde{u} e^{i(\vec{k} \cdot \Delta \vec{x}-\omega \Delta t)}
$$

Because all three components of the vector operator are independent, in terms of degrees of freedom, from each other, they can be evaluated separately. The stencil for each component is the standard six point stencil and can be evaluated as in the 3-form dispersion section. Assuming the longitudinal and transverse wave speeds are the same, the result is identical to the 3 -form result and is given in (5.112).

$$
\frac{\omega^{2}}{k^{2}}=c_{l}^{2} \frac{\left(1+\frac{1}{12}(k \Delta h)^{2}+\mathcal{O}\left(\Delta h^{4}\right)\right)}{\left(1-\frac{1}{12}(\omega \Delta t)^{2}+\mathcal{O}\left(\Delta t^{4}\right)\right)}
$$

\subsection{Linear Magnetohydrodynamic Wave Equation}

\subsubsection{Numerical stability}

The numerical stability for the magnetohydrodynamic wave equation is a combination of the stability for the acoustic term in the equation and the transverse wave term. The acoustic term has been analyzed previously in the Acoustic Vector Wave Equation section. The transverse wave term will be analyzed in this section.

The discrete form for the MHD term is given in (5.113) with eigenvalues $\kappa_{2}^{M H D}$.

$$
\mathbf{M}_{2} \vec{x}=\kappa_{2}^{M H D} \mathbf{M}_{\times}^{T} \mathbf{C}^{T} \mathbf{M}_{2} \mathbf{C M}_{\times} \vec{x}
$$


The matrix B for this term becomes (5.114).

$$
\mathbf{B}=2 \mathbf{I}-\Delta t^{2} \mathbf{M}_{2}^{-1} \mathbf{M}_{2} \mathbf{M}_{\times}^{T} \mathbf{C}^{T} \mathbf{M}_{2} \mathbf{C} \mathbf{M}_{\times}
$$

A simulation with sound speed $c_{l}$ set to zero will be stable if the timestep has the property (5.115).

$$
\Delta t \leq \frac{2}{\max \left(\kappa_{2}^{M H D}\right)}
$$

Combining this with the acoustic term eigenvalue the stability condition for the linear magnetohydrodynamics simulations is (5.116).

$$
\Delta t \leq \frac{2}{\sqrt{\left[\max \left(\kappa_{2}^{M H D}\right)+\max \left(\kappa_{2}^{\nabla \cdot}\right)\right]}}
$$

\subsubsection{Numerical dispersion}

The numerical dispersion for the acoustic term was derived in section for the acoustic vector wave equation. In this section the dispersion for the transverse wave term will be analyzed. Assuming a velocity in the x-direction on a two-dimensional uniform Cartesian mesh the MHD term stencil looks like Figure 5.9.

\begin{tabular}{l|l|l|l|l|l} 
& & & & & \\
\hline & & $-\frac{1}{4}$ & $-\frac{1}{2}$ & $-\frac{1}{4}$ & \\
\hline & $-\frac{1}{4}$ & $\frac{1}{2}$ & $\frac{3}{2}$ & $\frac{1}{2}$ & $-\frac{1}{4}$ \\
\hline & & $-\frac{1}{4}$ & $-\frac{1}{2}$ & $-\frac{1}{4}$ & \\
\hline & & & & &
\end{tabular}

Figure 5.9: 2 Dimensional MHD term Stencil

This stencil can be used to determine the dispersion by assuming a plane wave of 
the type (5.117).

$$
\vec{v}=\tilde{v} e^{i(\vec{k} \cdot \Delta \vec{x}-\omega \Delta t)}
$$

The coefficients and positions for the two-dimensional MHD term stencil are entered into the plane wave solution and expanded in a Taylor series about the wave vector $\vec{k}=k_{x} \Delta x \hat{i}+k_{y} \Delta y \hat{j}$ where the $\Delta x$ and $\Delta y$ terms are the phase difference form the central face. In two-dimensions the central face is an edge as shown in the stencil.

In the magnetohydrodynamic simulations a uniform magnetic flux density $\vec{B}_{0}$ is applied in the y-direction. This causes an asymmetry in the dispersion. The dispersion for the MHD term is listed in (5.118). The denominator comes from the analyses for the leap-frog time integration scheme and is identical to previous dispersion analyses.

$$
\frac{\omega^{2}}{|\vec{k}|^{2}}=\left|{\overrightarrow{v_{a}}}^{2}\right| \frac{\left(1+\frac{1}{3}\left(k_{x} \Delta x\right)^{2}+\frac{1}{12}\left(k_{y} \Delta y\right)^{2}+\mathcal{O}\left(\Delta h^{4}\right)\right)}{\left(1-\frac{1}{12}(\omega \Delta t)^{2}+\mathcal{O}\left(\Delta t^{4}\right)\right)}
$$

\subsubsection{Energy conservation}

Energy conservation for the magnetohydrodynamic equation involves three variables pressure, velocity and magnetic flux density. The energy equation given in (2.50) can be written in discrete form using 2-forms for the velocity and magnetic flux density and 3-forms for the pressure. The discrete magnetohydrodynamic energy equation for a domain with no energy entering is given in (5.119)

$$
\alpha^{T} \mathbf{M}_{3}^{\delta} \frac{\partial \alpha}{\partial t}+\beta^{T} \mathbf{M}_{2}^{\alpha} \frac{\partial \beta}{\partial t}+b^{T} \mathbf{M}_{2}^{\mu^{-1}} \frac{\partial b}{\partial t}=0
$$

The equations listed in (2.48) show the pressure and magnetic flux density are coupled to the velocity while the velocity is coupled to both the density and magnetic 
flux density. The pressure and magnetic flux density can therefore be integrated at half timesteps and the velocity integrated at whole time steps using the leap frog integration scheme in (5.2). The energy conservation equation for the linear magnetohydrodynamics equations is given in (5.120), the material $\delta$ and $\nu$ are defined as in the acoustic energy conservation.

$$
\begin{array}{r}
\left(\alpha^{n+1}\right)^{T} \mathbf{M}_{3}^{\delta} \alpha^{n+1}+\left(\beta^{n+\frac{1}{2}}\right)^{T} \mathbf{M}_{2}^{\nu} \beta^{n+\frac{1}{2}}+\left(b^{n+1}\right)^{T} \mathbf{M}_{2}^{\mu^{-1}} b^{n+1}+ \\
\left(\alpha^{n}\right)^{T} \mathbf{M}_{3}^{\delta} \alpha^{n}+\left(\beta^{n-\frac{1}{2}}\right)^{T} \mathbf{M}_{2}^{\nu} \beta^{n-\frac{1}{2}}+\left(b^{n}\right)^{T} \mathbf{M}_{2}^{\mu^{-1}} b^{n}=0
\end{array}
$$

The energy is conserved as in all of the other cases in this Chapter. 


\section{Chapter 6}

\section{Parallel Implementation}

The efficient solution of large scale simulations is the domain of massively parallel computing. The simulations presented in this dissertation range from simulation runs on a single processor to simulations that require hundreds of processors to run. Many computer architectures have been developed to enable these simulations. The four types of architectures are SISD (single instruction single data), SIMD (single instruction multiple data), MISD (multiple instruction single data), and MIMD (multiple instruction multiple data) [52]. The MIMD architecture comprises the majority of the slots in the top500 list of supercomputers [53]. These computers are typically several processors sharing memory on a single node that is then networked with many other nodes using fast network interface cards and network switches.

The instruction part of the architecture in the case of this dissertation is the computational model known as SAMD (single algorithm multiple data). Each processor runs the same algorithm using different data sets.

If the program running in parallel is not embarrassingly parallel, that is all data 
is not confined to the local processor, then data must be transferred to the other processors. The data is transfered to the other processors using MPI (message passing interface) [54]. Data is collected then sent to another or all other processors. The receiving processor will then receive the data and incorporate it into its own calculations. The message passing requires time and computation introducing overhead that limits the speedup of a simulation. The relative speedup $S_{p}$ of a simulation is defined as the ratio of the execution time on a single processor $T_{1}$ divided by the execution time on $p$ processors $T_{p} ; S_{p}=\frac{T_{1}}{T_{p}}$. Many other metrics for characterizing parallel implementations exist but this dissertation will use the above metrics.

\subsection{Parallel Toolkits}

Once the architecture and communication implementation are chosen, a parallel simulation code must be written. Gone are the days of hand crafting a linear solver to a given computer architecture. Several linear and non-linear solver packages are available, also called parallel toolkits for the large auxiliary functionality they provide. The toolkit used in this dissertation is PETSc, the portable extensible toolkit for scientific computing [27] [28] [26]. Another toolkit called Hypre [55] is a collection of scalable sparse linear system solvers. PETSC can use the Hypre toolkit code as both main solver and preconditioner, effectively combining the two toolkits. These toolkits build on MPI to define a higher level of abstraction. The communication is implemented for the user. The user then specifies sequential or parallel matrices and vectors that are partitioned automatically for the number of processors. Figure 6.1 shows a typical partitioning for four processors. 


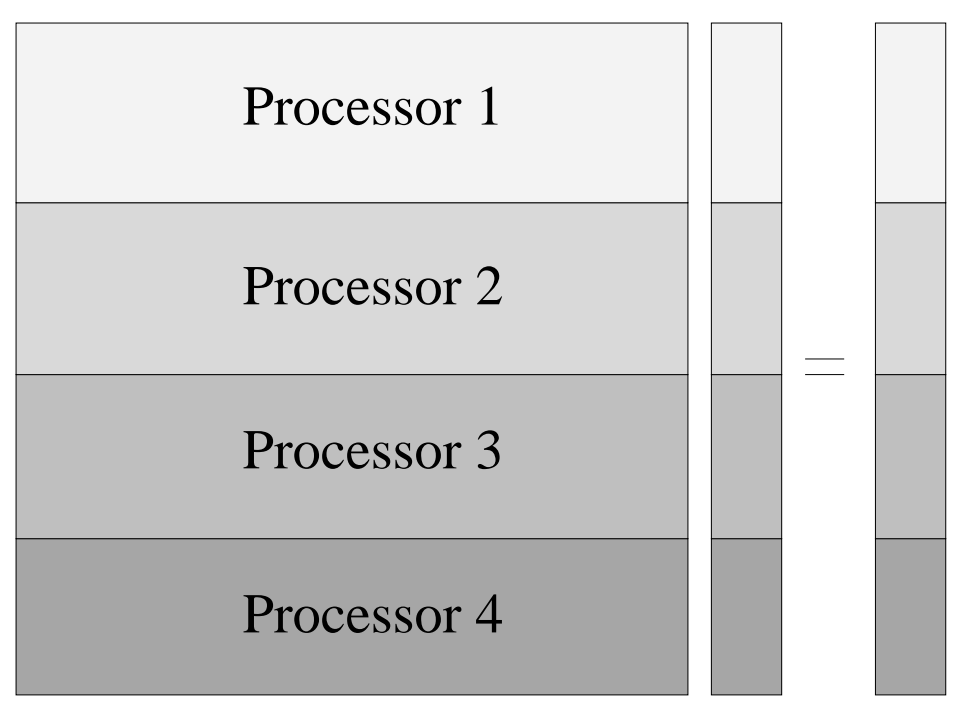

Figure 6.1: Matrix Partitioning

The different shades in the matrix partitioning show the parts of the matrix, solution vector and right hand side owned by each processor.

Both PETSc and Hypre implement many iterative solution methods. This dissertation uses the preconditioned conjugate gradient method discussed in Chapter 4. The version used in PETSc is parallel utilizing a partitioning as in Figure 6.1 to provide the parallel matrix vector multiplies needed for the algorithm.

\subsection{Python}

The interpreted computer language Python is part of the parallel implementation due to its utility. The language itself provides no parallel facility, but it is included in this chapter due to its simplicity of design and its ability to run c++ code. Python allows a programmer to write what is often referred to as functional pseudo-code. That is, code that can be easily understood due to its simple structure. 
In parallel the Python interpreter is run on every processor. Due to its interpreted nature the Python script runs slower than a typical compiled $\mathrm{C}$ or $\mathrm{C}++$ executable. This can be remedied by extending Python with $\mathrm{C} / \mathrm{C}++$ code. This process of extension allows the Python interpretor to run the $\mathrm{C} / \mathrm{C}++$ code as if it were Python code at native $\mathrm{C} / \mathrm{C}++$ speed. Several methods for extending Python such as Boost/Python [56] or SWIG [57] make the process relatively painless. The PETSC and Hypre toolkits mentioned above have both been compiled into Python modules. This provides a massively parallel interpreted linear solver system in some ways much like a massively parallel Matlab.

\subsection{Mesh Generation and Domain Decomposition}

The discrete differential forms framework is a finite element based method. The finite element method requires a mesh on which to define the basis functions. To implement an efficient finite element method in parallel, the domain of the problem is decomposed into smaller domains. Typically the number of sub-domains the domain is decomposed into is the same as the number of processors. Each processor receives its own domain, which speeds up the matrix generation, a significant amount of computation. Several methods are employed to decompose the domain. The method used in this dissertation is the k-way decomposition implemented in the METIS/ParMETIS code [58], [59], [60].

This method takes the full mesh like the mesh shown in Figure 6.2 and attempts to group edges and nodes to make a courser mesh. This algorithm is repeated until a very coarse mesh is created. This coarsest mesh can be partitioned easily. The 
algorithm is repeated until each of the child partitions are themselves partitioned into the requested number of partitions. The cylinder mesh shown in Figure 6.2 was partitioned using this algorithm into four partitions shown in Figure 6.3.

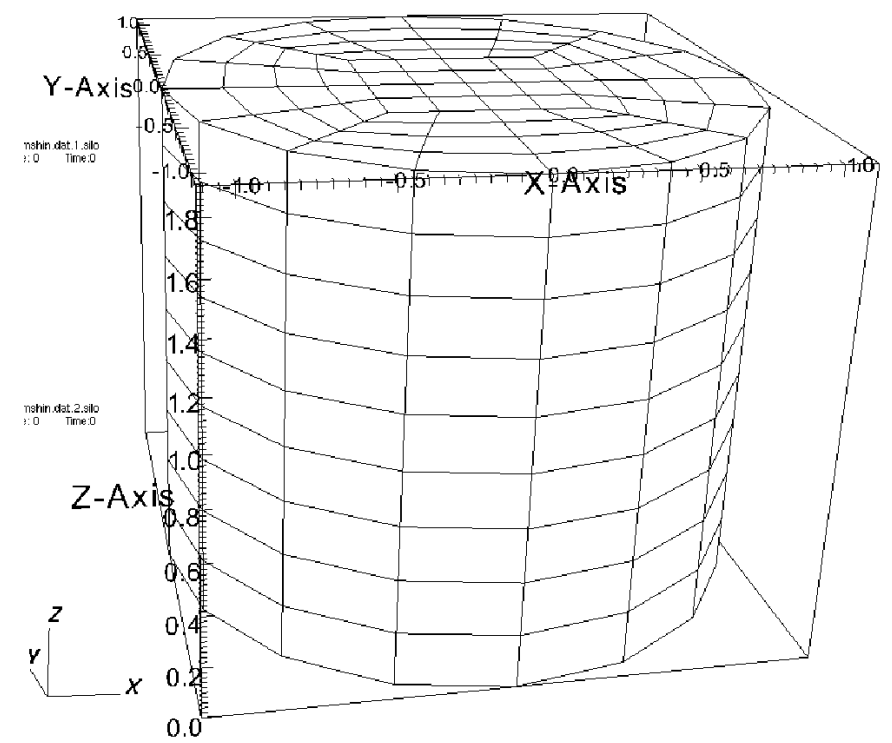

Figure 6.2: Cylinder Mesh 

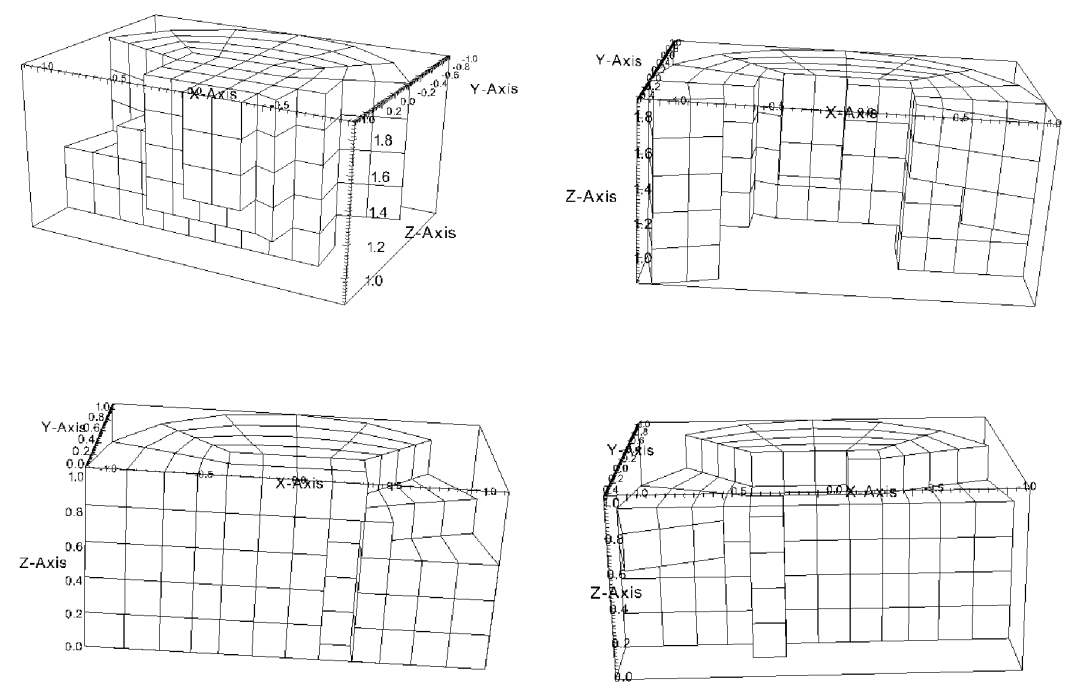

Figure 6.3: Cylinder Mesh Domain Decomposition 


\section{Chapter 7}

\section{Validation}

In this section validation simulations for the various wave equations are presented.

The finite element method allows unstructured grids with arbitrary element shapes. To demonstrate this feature, various meshes with hexahedral, tetrahedral and prism elements are used in the simulations. While the framework has been validated for the electrodynamics equations [22], the acoustics, elasticity and magnetohydrodynamics equations are untested. Therefore simulations in the frequency domain for small square and cubic grids will be analyzed to characterize the equations. Eigenvalue calculations in the time domain for larger unstructured domains will be presented to determine the accuracy of the framework. The dispersion relations given in Chapter 5 have shown the method is second order accurate. These simulations will confirm this accuracy. 


\subsection{Common simulation meshes}

This section contains some of the common meshes used in the simulations in the following sections. The two types of meshes common to the scalar and vector wave equation and the electrodynamics equations cavity simulations are the rectangular cavity and the spherical cavity. These meshes will be discretized using tetrahedrons and hexahedrons. Various meshes for the different simulations will be presented in the section in which they are introduced.

The rectangular mesh is a $29 m \times 25 m x 21 m$ rectangular region which is discretized using hexahedrons. The hexahedral mesh is constructed using a commercial grid generation package called Truegrid [61]. [62]. The mesh statistics for each mesh are listed in Table 7.1.

Table 7.1: Rectangular Cavity Meshes

\begin{tabular}{|c||c|c|c|}
\hline Mesh Type & Tetrahedral & Prism & Hexahedral \\
\hline \hline Nodes & 1331 & 1364 & 1331 \\
\hline Edges & 7930 & 4947 & 3630 \\
\hline Faces & 12600 & 5724 & 3300 \\
\hline Elements & 6000 & 2140 & 1000 \\
\hline
\end{tabular}

An unstructured spherical mesh is used for mesh refinement studies in all of the forms. A set of uniformly refined hexahedral meshes, Table 7.2, was constructed using Truegrid.

These spherical meshes all have a radius of $r=0.05855 \mathrm{~m}$. As stated in Chapter 3 the requirement for uniform refinement is the bound of the maximum and minimum angles in the mesh as $h \rightarrow 0$. The factor $\frac{h}{a}$ is a related parameter in each of the mesh series. The tetrahedral meshes are directly constructed from the series of 
Table 7.2: Spherical Cavity Hexahedral Refinement Meshes

\begin{tabular}{|c||c|c|c|c|}
\hline$\frac{\mathbf{h}}{\mathbf{a}}$ & $\frac{1}{4}$ & $\frac{1}{6}$ & $\frac{1}{8}$ & $\frac{1}{10}$ \\
\hline \hline Nodes & 321 & 997 & 2273 & 4341 \\
\hline Edges & 880 & 2832 & 6560 & 12640 \\
\hline Faces & 816 & 2700 & 6336 & 12300 \\
\hline Elements & 256 & 864 & 2048 & 4000 \\
\hline
\end{tabular}

hexahedral meshes. The series of tetrahedral meshes, Table 7.3, were constructed using each corresponding hexahedral grids' nodes with GEOMPACK [63] calculating the connectivity.

Table 7.3: Spherical Cavity Tetrahedral Refinement Meshes

\begin{tabular}{|c||c|c|c|c|}
\hline$\frac{\mathbf{h}}{\mathbf{a}}$ & $\frac{1}{4}$ & $\frac{1}{6}$ & $\frac{1}{8}$ & $\frac{1}{10}$ \\
\hline \hline Nodes & 321 & 997 & 2273 & 4431 \\
\hline Edges & 1952 & 6396 & 14944 & 28940 \\
\hline Faces & 3168 & 10584 & 24960 & 48600 \\
\hline Elements & 1536 & 5184 & 12288 & 24000 \\
\hline
\end{tabular}

A set of cylindrical meshes is used to test operator conformance. These meshes have radii of $a=2.23456 \mathrm{~m}$ and lengths of $L=1.9 \mathrm{~m}$. The number of nodes, edges, faces and elements is shown in Table 7.4.

Table 7.4: Cylindrical Cavity Meshes

\begin{tabular}{|c||c|c|c|}
\hline Mesh Type & Tetrahedral & Prism & Hexahedral \\
\hline \hline Nodes & 890 & 873 & 890 \\
\hline Edges & 5534 & 3080 & 2481 \\
\hline Faces & 8986 & 3488 & 2312 \\
\hline Elements & 4341 & 1280 & 720 \\
\hline
\end{tabular}




\subsection{Operator conformance}

In the previous chapters discrete operators for vector identities as well as discrete stiffness matrices were introduced. These operators can be used to verify the accuracy of the finite element method and the correctness of the grid. The tables below are generated for the rectangular grids shown in Table 7.1 and the cylindrical grids shown in Table 7.4.

To test the vector identities listed in (2.78), the discrete versions are formed for the natural Curl-Grad and Div-Curl operators (7.1) and the adjoint Curl-Grad and Div-Curl (7.2) operators.

$$
\begin{aligned}
& \mathbf{C G}=0 \\
& \mathbf{D C}=0 \\
& \tilde{\mathbf{C}} \tilde{\mathbf{G}}=0 \\
& \tilde{\mathbf{D}} \tilde{\mathbf{C}}=0
\end{aligned}
$$

The natural operators will be zero independent of element type, grid structure or grid distortion. Because the adjoint operators involve a matrix inversion, these identities will only be zero in an approximate sense depending on the accuracy of the solution. The absolute error for the natural and adjoint operators for the rectangular grid are shown in Table 7.5 and for the cylindrical grid in Table 7.6.

In Chapter 3 it was shown that the stiffness matrices can be constructed using either the second order wave equation or combining the first order wave equations in the scalar wave equation, electrodynamics and vector acoustic wave equation sections. The results for the absolute difference between the two stiffness matrix creation 
Table 7.5: Rectangular Mesh Vector Operator Conformance

\begin{tabular}{|c|c||c|c|c|}
\hline Operator & Discrete Form & Tetrahedral & Prism & Hexahedral \\
\hline \hline$\nabla \times \nabla$ & $\mathbf{C G}=0$ & 0.0 & 0.0 & 0.0 \\
\hline$\nabla \times \nabla$ & $\mathbf{M}_{1}^{-1} \mathbf{C}^{T} \mathbf{D}^{T} \mathbf{M}_{3}=0$ & $8.861474 \mathrm{e}-15$ & $4533739 \mathrm{e}-16$ & $2.793370 \mathrm{e}-16$ \\
\hline$\nabla \cdot \nabla \times$ & $\mathbf{D C}=0$ & 0.0 & 0.0 & 0.0 \\
\hline$\nabla \cdot \nabla \times$ & $\mathbf{M}_{0}^{-1} \mathbf{G}^{T} \mathbf{C}^{T} \mathbf{M}_{2}=0$ & $2.652972 \mathrm{e}-15$ & $1.886097 \mathrm{e}-15$ & $1.817464 \mathrm{e}-15$ \\
\hline
\end{tabular}

Table 7.6: Cylindrical Mesh Vector Operator Conformance

\begin{tabular}{|c|c||c|c|c|}
\hline Mesh Type & Discrete Form & Tetrahedral & Prism & Hexahedral \\
\hline \hline$\nabla \times \nabla$ & $\mathbf{C G}=0$ & 0.0 & 0.0 & 0.0 \\
\hline$\nabla \times \nabla$ & $\mathbf{M}_{1}^{-1} \mathbf{C}^{T} \mathbf{D}^{T} \mathbf{M}_{3}=0$ & $1.433930 \mathrm{e}-10$ & $2.024319 \mathrm{e}-11$ & $5.326277-12$ \\
\hline$\nabla \cdot \nabla \times$ & $\mathbf{D C}=0$ & 0.0 & 0.0 & 0.0 \\
\hline$\nabla \cdot \nabla \times$ & $\mathbf{M}_{0}^{-1} \mathbf{G}^{T} \mathbf{C}^{T} \mathbf{M}_{2}=0$ & $4.648776 \mathrm{e}-11$ & $7.612939 \mathrm{e}-11$ & $3.053943 \mathrm{e}-11$ \\
\hline
\end{tabular}

methods are shown in (7.3).

$$
\begin{gathered}
\mathbf{K}_{0} \equiv \mathbf{G}^{T} \mathbf{M}_{1} \mathbf{G} \\
\mathbf{K}_{1} \equiv \mathbf{C}^{T} \mathbf{M}_{2} \mathbf{C} \\
\mathbf{K}_{2} \equiv \mathbf{D}^{T} \mathbf{M}_{3} \mathbf{D}
\end{gathered}
$$

The comparison of the two methods for the stiffness matrix creation are shown for the rectangular grid in Table 7.7 and the cylindrical grid in Table 7.8.

Table 7.7: Rectangular Mesh Stiffness Matrix Conformance

\begin{tabular}{|c|c||c|c|c|}
\hline Operator & Discrete Form & Tetrahedral & Prism & Hexahedral \\
\hline \hline$\nabla \cdot \nabla$ & $\mathbf{G}^{T} \mathbf{M}_{1} \mathbf{G}-\mathbf{K}_{0}=0$ & $4.119507 \mathrm{e}-16$ & $3.618687-16$ & $3.638330-16$ \\
\hline$\nabla \times \nabla \times$ & $\mathbf{C}^{T} \mathbf{M}_{2} \mathbf{C}-\mathbf{K}_{1}=0$ & $3.468225 \mathrm{e}-16$ & $1.072193 \mathrm{e}-16$ & $4.665859-16$ \\
\hline$\nabla \nabla \cdot$ & $\mathbf{D}^{T} \mathbf{M}_{3} \mathbf{D}-\mathbf{K}_{2}=0$ & $3.758319 \mathrm{e}-16$ & $2.155468 \mathrm{e}-16$ & $2.099643-16$ \\
\hline
\end{tabular}

All of the comparisons are zero to machine precision showing the two methods for constructing the stiffness matrices are identical. If a grid is improperly constructed then one or more of the above tests will fail. These tests provide a necessary but 
Table 7.8: Cylindrical Mesh Stiffness Matrix Conformance

\begin{tabular}{|c|c||c|c|c|}
\hline Operator & Discrete Form & Tetrahedral & Prism & Hexahedral \\
\hline \hline$\nabla \cdot \nabla$ & $\mathbf{G}^{T} \mathbf{M}_{1} \mathbf{G}-\mathbf{K}_{0}=0$ & $3.780916 \mathrm{e}-16$ & $3.808475 \mathrm{e}-16$ & $3.770671 \mathrm{e}-16$ \\
\hline$\nabla \times \nabla \times$ & $\mathbf{C}^{T} \mathbf{M}_{2} \mathbf{C}-\mathbf{K}_{1}=0$ & $3.008296-16$ & $3.220813 \mathrm{e}-16$ & $3.914080 \mathrm{e}-16$ \\
\hline$\nabla \nabla \cdot$ & $\mathbf{D}^{T} \mathbf{M}_{3} \mathbf{D}-\mathbf{K}_{2}=0$ & $4.323948 \mathrm{e}-16$ & $2.005644 \mathrm{e}-16$ & $2.200214 \mathrm{e}-16$ \\
\hline
\end{tabular}

not sufficient condition for proper construction of the discrete differential forms finite element system. To prove the system is accurate, eigenvalue calculations for the various wave equations are performed below.

\subsection{Scalar Wave Equation}

The scalar wave equation is typically solved using 0 -forms or nodal finite elements. Raviart and Thomas proposed an alternative formulation using a mixed finite element method comprised of 2-forms and 3-forms in two dimensions. Both of these formulations will be used to solve a linear acoustic scalar wave equation for the second order pressure and density wave equations.

\subsubsection{Space characterization}

One of the requirements for a discrete differential form is a discrete space that are subspaces of the corresponding Hilbert spaces. In this section the discrete subspaces for $\mathcal{H}(\mathrm{grad})$ and $L^{2}$ will be examined for the 0 -form and 3-form respectively.

The discrete scalar wave equation with wave speed $c_{l}=1$ in the frequency domain is shown in (7.4)

$$
\nabla \cdot(\nabla \psi)=\omega^{2} \psi
$$


where $\psi$ represents the pressure or density and $\omega^{2}=k^{2}$ is the angular frequency eigenvalue. The domain of the problem is a unit cube with dimensions $a \times b \times c=$ $1 m \times 1 m \times 1 m$ discretized using a $9 x 9 x 9$ zone hexahedral grid with 1000 nodes and 729 elements. The eigenvalues are given by (7.5).

$$
\omega_{m n l}^{2}=c_{l}^{2} \pi^{2}\left(\frac{m^{2}}{a^{2}}+\frac{n^{2}}{b^{2}}+\frac{l^{2}}{c^{2}}\right)
$$

The specific values of $m, n, l$ depend on the boundary conditions imposed on the domain.

For the first experiment the eigenvalues will be compared for the homogeneous essential boundary conditions of the 0-form Div-Grad operator and the homogeneous natural boundary conditions of the 3-form Div-Grad equation(7.6).

$$
\begin{aligned}
& \nabla \cdot\left(\nabla \psi_{0}\right)=\omega_{0}^{2} \psi_{0} \text { with } \psi_{0}=0 \text { on } \Gamma \\
& \nabla \cdot\left(\nabla \psi_{3}\right)=\omega_{3}^{2} \psi_{3} \text { with } \psi_{3}=0 \text { on } \Gamma
\end{aligned}
$$

In this case the values for the coefficients $m, n, l$ are given in (7.7) with corresponding 0 -form $\psi_{0}$ and 3 -form $\psi_{3}$ eigenvectors given in (7.8).

$$
\begin{gathered}
m=1,2,3,4 \ldots \\
n=1,2,3,4 \ldots \\
l=1,2,3,4 \ldots \\
\psi_{0}=\psi_{3}=A_{m, n, l} \sin \left(\frac{m \pi}{a}\right) \sin \left(\frac{n \pi}{a}\right) \sin \left(\frac{l \pi}{a}\right)
\end{gathered}
$$

The five smallest eigenvalues are listed in Table 7.9. In the case of the 0-form the essential boundary conditions are used, while in the case of the 3-form the natural boundary conditions are applied resulting in the same boundary condition. 
Table 7.9: Div-Grad Eigenvalues with Dirichlet Boundary Conditions

\begin{tabular}{|c||c|c|c|c|c|}
\hline mode & $\omega^{2}$ true & $\omega_{0}^{2}$ & \% error & $\omega_{3}^{2}$ & \% error \\
\hline \hline 1 & 29.608813 & 29.910665 & 1.019465 & 29.910664 & 1.019461 \\
\hline 2 & 59.217626 & 61.046938 & 3.089133 & 61.046943 & 3.089142 \\
\hline 3 & 88.826440 & 92.183211 & 3.779022 & 92.183223 & 3.779036 \\
\hline 4 & 108.565648 & 117.140453 & 7.898267 & 117.140422 & 7.898239 \\
\hline 5 & 118.435253 & 123.319483 & 4.123967 & 123.319504 & 4.123985 \\
\hline
\end{tabular}

The domain was chosen so that all of the eigenvalues for the discrete domain could be calculated. For the problem above the null space of the discrete operator should be empty which is the case for both discrete equations. The range space for the two operators is different due to the different discretizations. The dimension of each space is shown in Table 7.10 where $\operatorname{Dim}($ Domain $)=\operatorname{Dim}($ Range $)+\operatorname{Dim}($ Null $)$.

Table 7.10: Div-Grad Discrete Space Dimensions for Dirichlet Boundary Conditions

\begin{tabular}{|c||c|c|}
\hline Space & 0-form Div-Grad & 3-form Div-Grad \\
\hline \hline Dim(Range) & 512 & 729 \\
\hline Dim(Null) & 0 & 0 \\
\hline Dim(Domain) & 512 & 729 \\
\hline
\end{tabular}

The eigenvalues are also compared for the homogeneous Neumann boundary conditions which correspond to the natural boundary condition of the the 1 Div-Grad operator and the essential boundary conditions of the 3-form Div-Grad operator (7.9)

$$
\begin{aligned}
& \nabla \cdot\left(\nabla \psi_{0}\right)=\omega_{0}^{2} \psi_{0} \text { with } \nabla \psi_{0} \cdot \hat{n}=0 \text { on } \Gamma \\
& \nabla \cdot\left(\nabla \psi_{3}\right)=\omega_{3}^{2} \psi_{3} \text { with } \nabla \psi_{3} \cdot \hat{n}=0 \text { on } \Gamma
\end{aligned}
$$

In this case the values for the coefficients $m, n, l$ for the eigenvalues (7.5) are given in $(7.10)$

$$
m=0,1,2,3,4 \ldots
$$




$$
\begin{gathered}
n=0,1,2,3,4 \ldots \\
l=0,1,2,3,4 \ldots
\end{gathered}
$$

The eigenvectors corresponding to the homogeneous Neumann boundary condition are listed in (7.11).

$$
\psi_{0}=\psi_{3}=A_{m, n, l} \cos \left(\frac{m \pi}{a}\right) \cos \left(\frac{n \pi}{a}\right) \cos \left(\frac{l \pi}{a}\right)
$$

Table 7.11: Div-Grad Eigenvalues with Neumann Boundary Conditions

\begin{tabular}{|c||c|c|c|c|c|}
\hline mode & $\omega^{2}$ true & $\omega_{0}^{2}$ calc & \% error & $\omega_{3}^{2}$ calc & \% error \\
\hline \hline 1 & 9.869604 & 9.970221 & 1.019461 & 9.970222 & 1.019465 \\
\hline 2 & 19.739209 & 19.940442 & 1.019461 & 19.940443 & 1.019465 \\
\hline 3 & 29.608813 & 29.910664 & 1.019461 & 29.910665 & 1.019465 \\
\hline 4 & 39.478418 & 41.106501 & 4.123984 & 41.106495 & 4.123967 \\
\hline 5 & 49.348022 & 51.076722 & 3.503080 & 51.076716 & 3.503067 \\
\hline
\end{tabular}

The dimensions of the total domain, range and null spaces are shown in Table 7.12. In this case the null space is not empty due to the single zero eigenvalue when $m=n=l=0$.

Table 7.12: Div-Grad Discrete Space Dimensions for Neumann Boundary Conditions

\begin{tabular}{|c||c|c|}
\hline Space & 0-form Div-Grad & 3-form Div-Grad \\
\hline \hline Dim(Range) & 999 & 728 \\
\hline Dim(Null) & 1 & 1 \\
\hline Dim(Domain) & 1000 & 729 \\
\hline
\end{tabular}

Both the 0 -form and 3-form scalar wave equation discretizations conform to the proper domain, range and null space dimensions for the two types of boundary conditions listed above. 


\subsubsection{Spherical cavity}

The 0 -form pressure and 3-form density scalar wave equations have the solution (7.12) for a spherical region with radius $a$. This section will solve the scalar wave equation to determine the frequencies for a closed spherical acoustic cavity in the time domain. The entirely closed cavity has Neumann boundary conditions over the entire region so that the normal component of the gradient of the pressure is zero $\nabla P \cdot \hat{n}=0$

$$
\begin{aligned}
\psi(r, \theta, \phi) & =A_{m n p} \hat{J}_{n}(\beta r) P_{n}^{m}(\cos (\theta))[C \cos (m \theta)+D \sin (m \theta)] \\
\nabla \psi & =0 \text { on } \Gamma
\end{aligned}
$$

The frequencies (7.14) for the cavity are the $\operatorname{roots} \beta=\frac{\pi \alpha_{n, p}}{a}$ of the derivative of the Spherical Bessel function $\hat{J}_{m}^{\prime}(\beta r)$ due to the Neumann boundary conditions.

$$
\begin{aligned}
f & =\frac{c_{l}}{2} \frac{\alpha_{n, p}}{a} \\
m & =0,1,2,3, \ldots \\
n & =0,1,2,3, \ldots \\
p & =1,2,3, \ldots
\end{aligned}
$$

A series of related meshes for each of the hexahedral and tetrahedral grids as discussed in Section 7.1 is used to determine the convergence of the wave equation. Grids with prism domain decompositions are not used due to the geometry. A time domain simulation with an excitation of the second derivative of a Gaussian over $5 \%$ of the degrees of freedom results in a power spectrum as in Figure 7.1. This simulation was run for 50 periods of the lowest mode. The Gaussian pulses used are short duration and are introduced at the beginning of the simulation. 
The lowest exact frequencies for the scalar wave equation with $c_{l}=1$ are shown in Table 7.13. Due to stability constraints the total number of timesteps taken increases with the grid size so that all of the simulations run for different total cpu times.

Table 7.13: Exact Lowest Acoustic Frequencies

\begin{tabular}{|c|c|c|c|c|c|}
\hline$f_{m 11}$ & $f_{m 12}$ & $f_{002}$ & $f_{m 31}$ & $f_{m 21}$ & $f_{m 41}$ \\
\hline \hline 5.6584 & 9.0845 & 12.2143 & 12.2701 & 15.3493 & 16.1477 \\
\hline
\end{tabular}

The second order 0-form discrete acoustic wave equation (3.93) is used to determine the acoustic frequencies of the spherical cavity in the time domain. A conjugate gradient method is used to solve the mass consistent matrix $\mathbf{M}_{0}$. Ten degrees of freedom are chosen at random for which the field data is written out at every timestep. This data is zero extended to a power of two, the Fourier transform applied and a power spectrum created. The power spectra for the series of hexahedral grids shown in Table 7.2 is shown in Figure 7.1. The exact frequencies are represented in the spectra as vertical lines. As the grids are refined the peak representing the computed frequency approaches the true frequency.

The power spectra were also calculated for the tetrahedral grids listed in Table 7.3. These spectra are shown in Figure 7.2. The errors for both the hexahedral and tetrahedral 0-form acoustic wave equation time domain frequency simulations are shown in Table 7.14. A plot of the logarithm of the error versus the logarithm of $\frac{h}{a}$ for the hexahedral and tetrahedral grids is shown in Figure 7.3.

A least squares fit is be applied to the data and is also shown in Figure 7.3. The results show the slope for the hexahedral grid series is 2.26 while the slope for the tetrahedral grids is 1.97. Both show the simulations give errors that are second order 

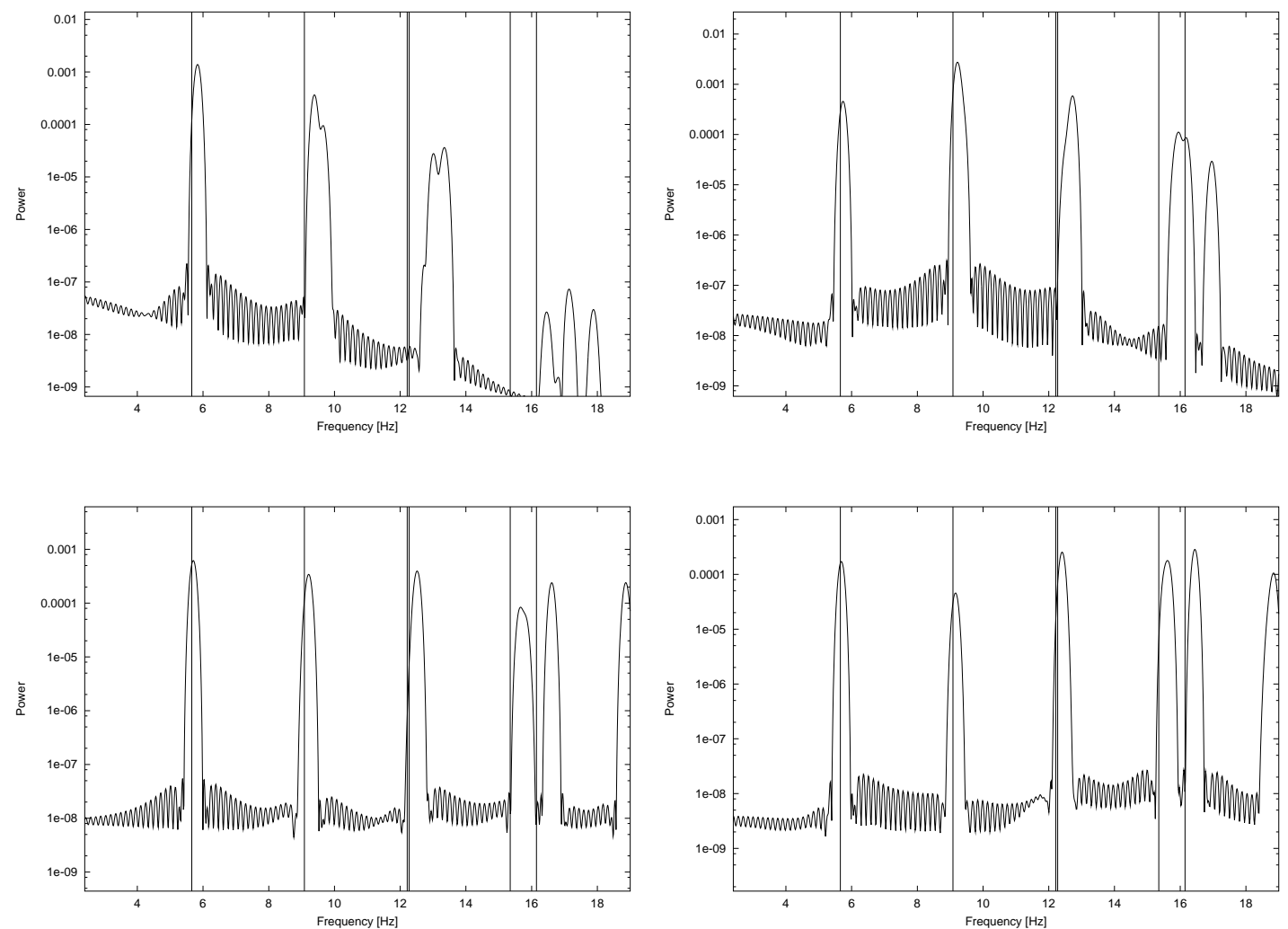

Figure 7.1: Power Spectra for the 0-form acoustic simulation for the series of hexahedral grids $\left(\frac{h}{a}=\frac{1}{4}, \frac{1}{6}, \frac{1}{8}, \frac{1}{10}\right.$; left to right,top to bottom)

Table 7.14: 0-form Acoustic Refinement Results

\begin{tabular}{|c|c|c|c|c|c|c|}
\hline Element & Parameter & Mesh 1 & Mesh 2 & Mesh 3 & Mesh 4 & Slope \\
\hline \hline Hex & Edge Length & 0.025 & 0.017 & 0.013 & 0.010 & 2.26 \\
\hline & Error & 0.176 & 0.076 & 0.048 & 0.022 & \\
\hline Tet & Edge Length & 0.034 & 0.025 & 0.020 & 0.017 & 1.97 \\
\hline & Error & 0.179 & 0.080 & 0.053 & 0.030 & \\
\hline
\end{tabular}



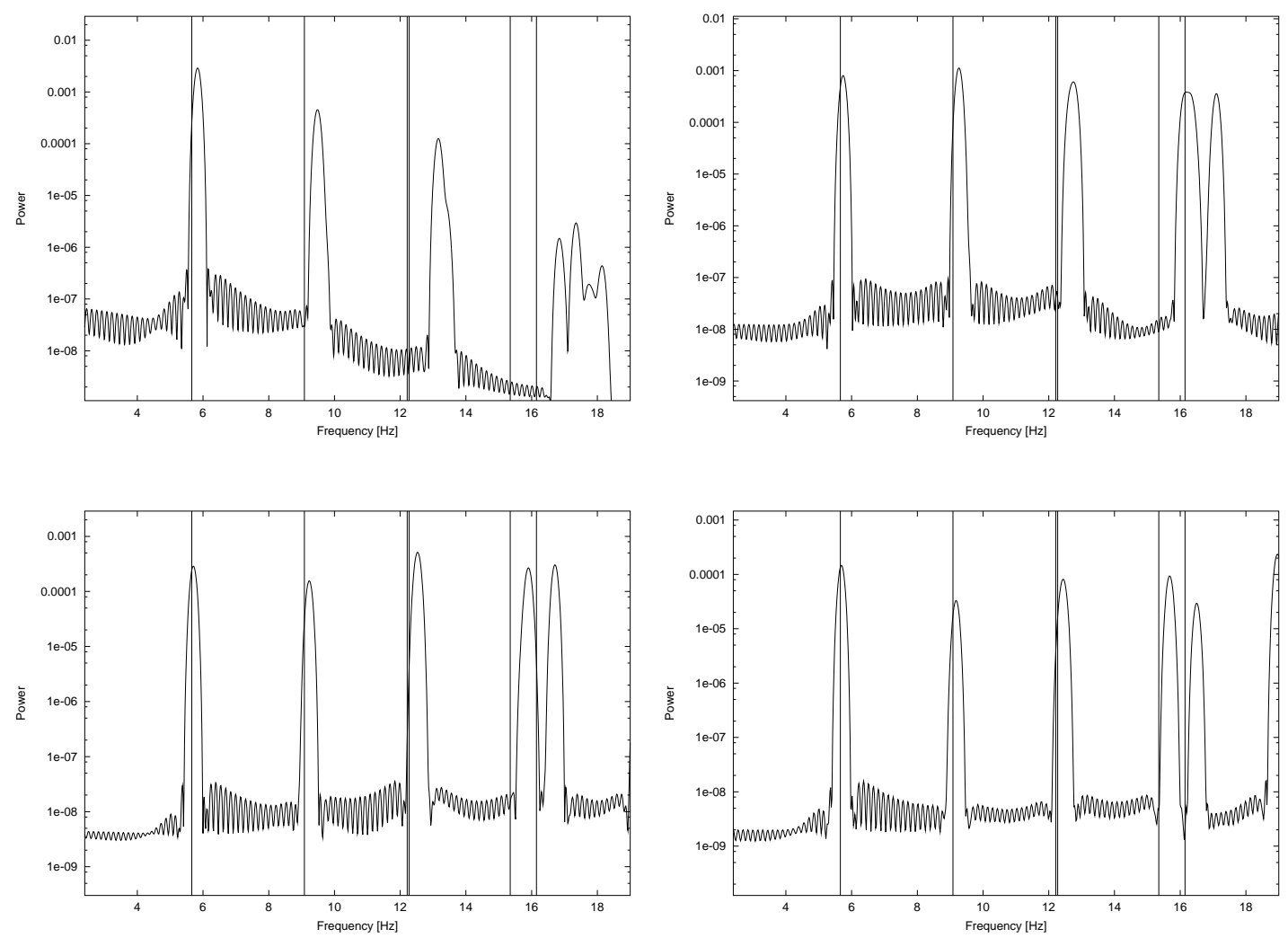

Figure 7.2: Power Spectra for the 0-form acoustic simulation for the series of tetrahedral grids $\left(\frac{h}{a}=\frac{1}{4}, \frac{1}{6}, \frac{1}{8}, \frac{1}{10}\right.$; left to right,top to bottom) 
accurate. The number of degrees of freedom is the same in both series of grids so that the simulations should give the same slope. The discrepancy in the slopes is due to the solution method.

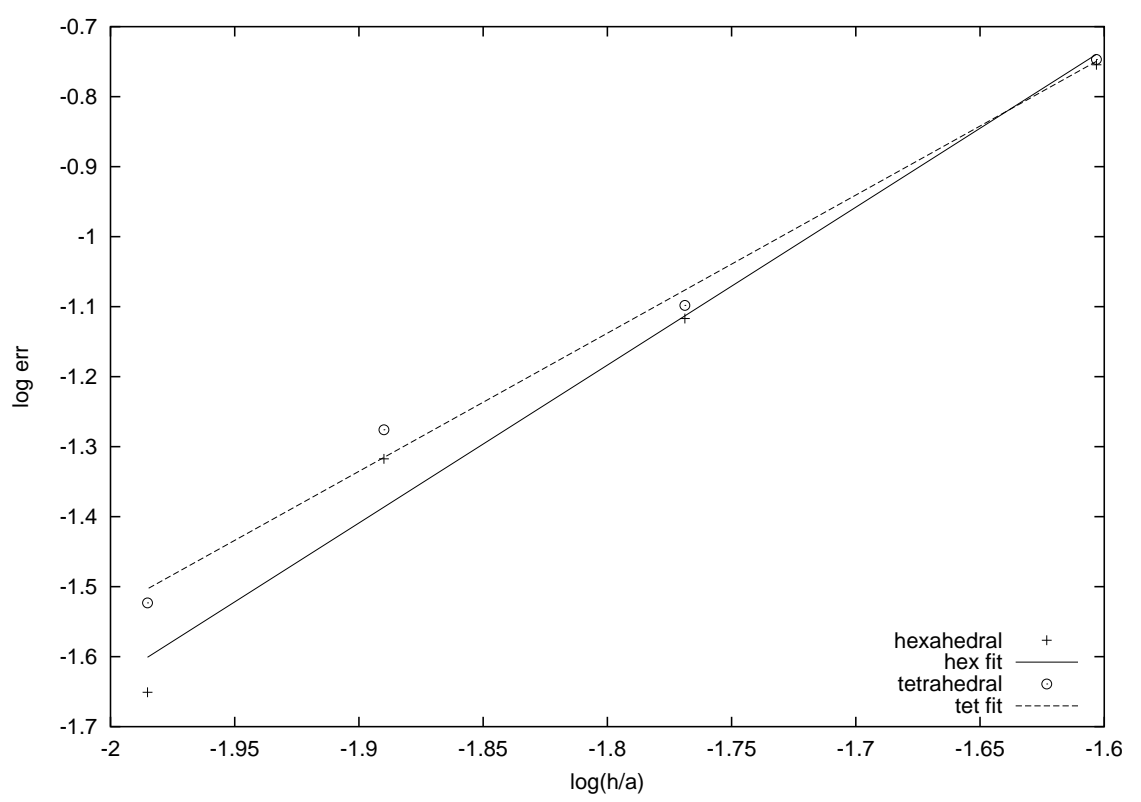

Figure 7.3: Mesh Refinement Results for 0-form Pressure Wave Equation

The second order 3-form discrete acoustic wave equation (3.100) is used to determine the acoustic frequencies of the spherical cavity in the time domain. The method used to solve this wave equation is the same as in the 0 -form acoustic wave equation. The power spectra for the series of hexahedral grids shown in Table 7.2 is shown in Figure 7.4. The exact frequencies are represented in the spectra as vertical lines. As the grids are refined the peak representing the computed frequency approaches the true frequency.

The power spectra were also calculated for the tetrahedral grids listed in Table 7.3. These spectra are shown in Figure 7.5.

The 3-form acoustic wave equation error results show a different trend than the 0- 

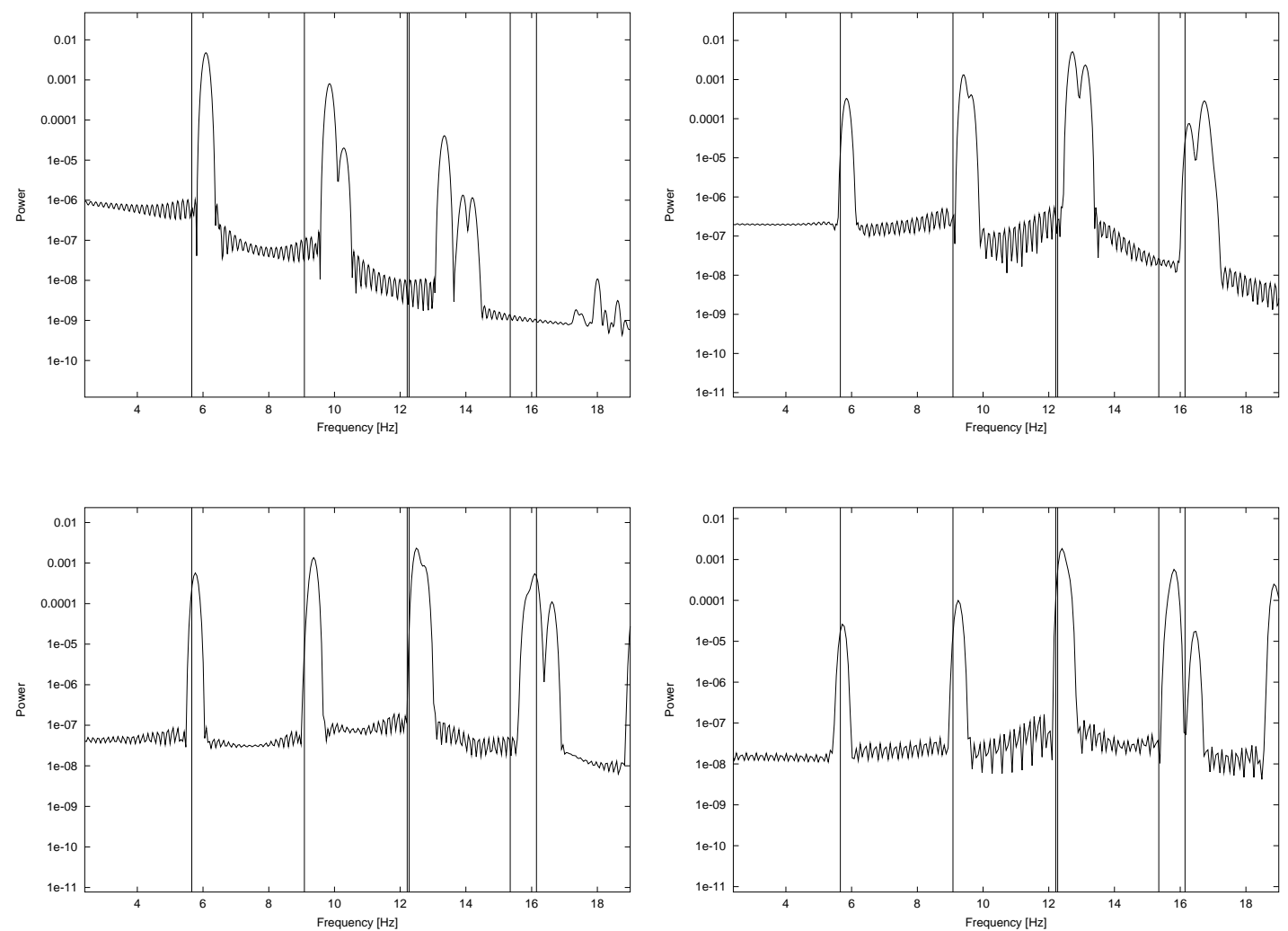

Figure 7.4: Power Spectra for the 3-form acoustic simulation for the series of hexahedral grids $\left(\frac{h}{a}=\frac{1}{4}, \frac{1}{6}, \frac{1}{8}, \frac{1}{10}\right.$; left to right,top to bottom) 

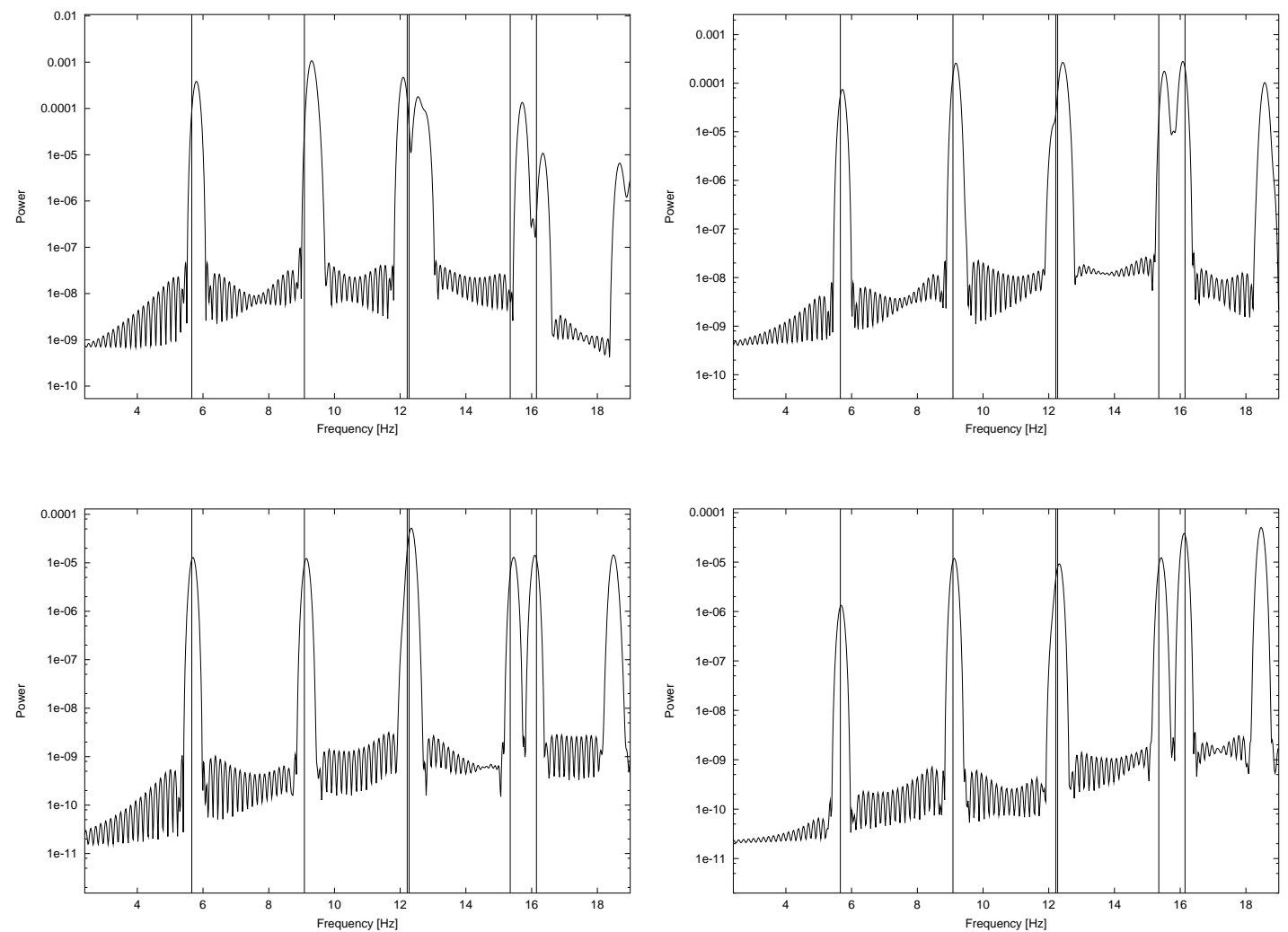

Figure 7.5: Power Spectra for the 3-form acoustic simulation for the series of tetrahedral grids ( $\frac{h}{a}=\frac{1}{4}, \frac{1}{6}, \frac{1}{8}, \frac{1}{10} ;$ left to right,top to bottom) 
form acoustic wave equation. The tetrahedrons are constructed from the hexahedrons. Each hex is decomposed into six tetrahedrons giving six times as many tetrahedrons than hexahedrons for a mesh with an equivalent $\frac{h}{a}$.

Table 7.15: 3-form Acoustic Refinement Results

\begin{tabular}{|c|c|c|c|c|c|c|}
\hline Element & Parameter & Mesh 1 & Mesh 2 & Mesh 3 & Mesh 4 & Slope \\
\hline \hline Hex & Edge Length & 0.025 & 0.017 & 0.013 & 0.010 & 2.25 \\
\hline & Error & 0.436 & 0.179 & 0.106 & 0.058 & \\
\hline Tet & Edge Length & 0.034 & 0.025 & 0.020 & 0.017 & 2.18 \\
\hline & Error & 0.139 & 0.056 & 0.038 & 0.028 & \\
\hline
\end{tabular}

The plot of the logarithm of error versus the logarithm of $\frac{h}{a}$ is shown in Figure 7.6. A least squares fit was applied to the data giving a slope of 2.25 for the hexahedron series and 2.18 for the tetrahedron series showing that the method is second order accurate. The tetrahedral grid errors are much lower than the hexahedral grid for the same $\frac{h}{a}$ due to the much larger number of degrees of freedom in the tetrahedral grids.

\subsection{Electrodynamic Vector Wave Equation}

\subsubsection{Space characterization}

In this section we look at the eigenvalues of the two different discretizations for the Helmholtz equation. The eigenvalues and eigenvectors will be calculated for the same 10x10x10 hexahedral grid used in Section 7.3.1. This mesh has 729 elements, 1000 nodes elements of which 512 are internal, 2700 edges of which 1728 are internal and 2430 faces of which 1944 are internal. The actual eigenvalues are $\omega_{m n}^{2}=\frac{1}{\epsilon \mu} \pi^{2}\left(\frac{m^{2}}{a^{2}}+\right.$ 


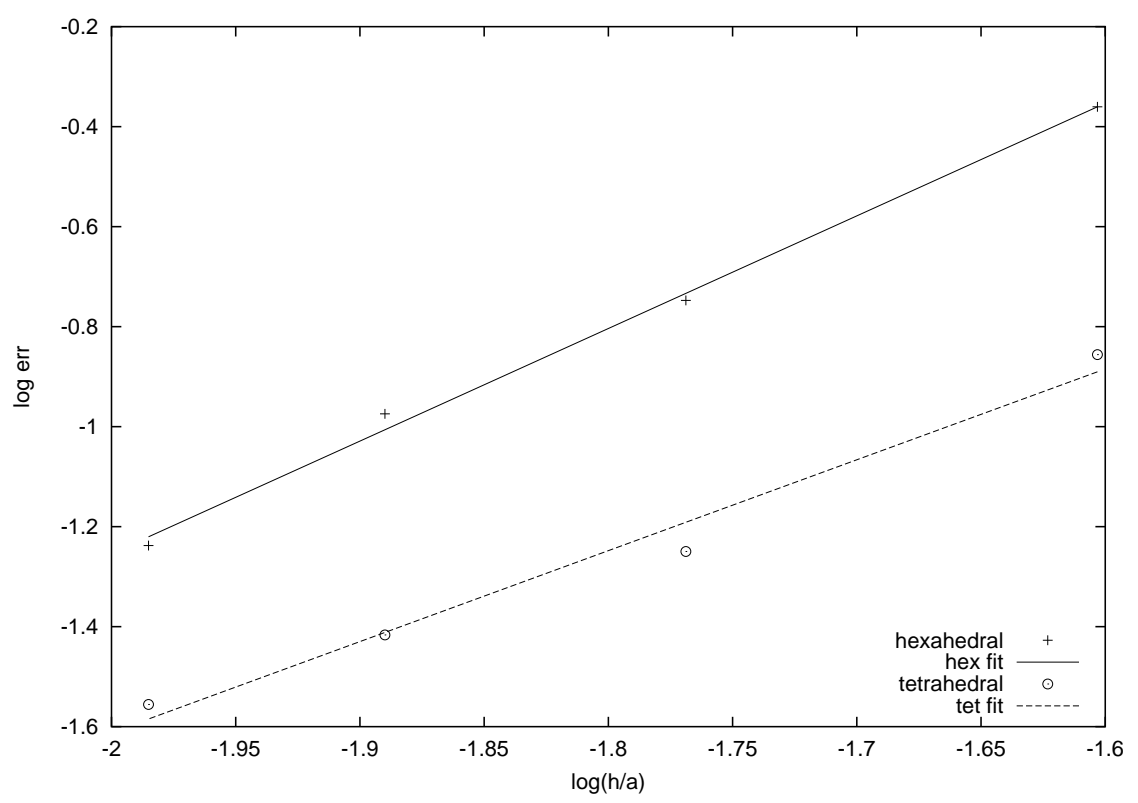

Figure 7.6: Mesh Refinement Results for 3-form Density Wave Equation

$\left.\frac{n^{2}}{b^{2}}+\frac{l^{2}}{c^{2}}\right)$ where $a, b, c$ are the dimensions of the box and $m, n, l$ are the mode numbers. For both of the cases listed below, the coefficients $m, n, l$ for the eigenvalues are given in $(7.15)$.

$$
\begin{array}{r}
m=0,1,2,3,4 \ldots \\
n=0,1,2,3,4 \ldots \\
l=0,1,2,3,4 \ldots \\
\text { except } m=n=l=0
\end{array}
$$

The domain for the following simulations is a unit cube.

The eigenvalues will be compared for the essential boundary conditions of the edge Curl-Curl operator corresponding to the homogeneous Dirichlet boundary conditions and essential boundary conditions of the face Curl-Curl equations corresponding to 
the homogeneous Dirichlet boundary condition (7.16) with $\epsilon=\mu=1$.

$$
\begin{gathered}
\nabla \times \nabla \times \vec{\psi}_{1}=\omega_{1}^{2} \vec{\psi}_{1} \text { with } \vec{\psi}_{1} \times \hat{n}=0 \text { on } \Gamma \\
\nabla \times \nabla \times \vec{\psi}_{2}=\omega_{2}^{2} \vec{\psi}_{2} \text { with } \vec{\psi}_{2} \cdot \hat{n}=0 \text { on } \Gamma
\end{gathered}
$$

The eigenvalues for these two equations are compared in Table 7.16. The table shows the agreement with the true eigenvalues and with each other for the different discretizations and different imposed boundary conditions of the vector Helmholtz equation.

Table 7.16: Curl-Curl Eigenvalues with Dirichlet Boundary Conditions

\begin{tabular}{|c|c||c|c|c|c|}
\hline mode & $\omega_{1,2}^{2}$ true & $\omega_{1}^{2}$ calc & $\%$ error & $\omega_{2}^{2}$ calc & \% error \\
\hline \hline 1 & 19.739209 & 19.940443 & 1.019465 & 19.940443 & 1.019465 \\
\hline 2 & 29.608813 & 29.910665 & 1.019465 & 29.910665 & 1.019465 \\
\hline 3 & 49.348022 & 51.076716 & 3.503067 & 51.076716 & 3.503067 \\
\hline 4 & 59.217626 & 61.046938 & 3.089133 & 61.046938 & 3.089133 \\
\hline 5 & 78.956835 & 82.212989 & 4.123967 & 82.212989 & 4.123967 \\
\hline
\end{tabular}

The difference between the two discretizations can be seen in Table 7.17. For the homogeneous Dirichlet boundary conditions the dimension of the non-zero eigenvalues is the same, while the dimension of the null space is different leading to the different total dimension size.

Table 7.17: Curl-Curl Discrete Space Dimensions for Dirichlet Boundary Conditions

\begin{tabular}{|c||c|c|}
\hline Space & 1-form Curl-Curl & 2-form Curl-Curl \\
\hline \hline Dim(Range) & 1216 & 1216 \\
\hline Dim(Null) & 512 & 728 \\
\hline Dim(Domain) & 1728 & 1944 \\
\hline
\end{tabular}

Alternatively the eigenvalues and eigenvectors can be determined for the natural boundary conditions of the edge Curl-Curl corresponding to the homogeneous Neu- 
mann boundary conditions and the natural boundary conditions of the face Curl-Curl equation corresponding to the homogeneous Neumann boundary conditions (7.17).

$$
\begin{gathered}
\nabla \times \nabla \times \vec{\psi}_{1}=\omega_{1}^{2} \vec{\psi}_{1} \text { with } \nabla \times \vec{\psi}_{1} \times \hat{n}=0 \text { on } \Gamma \\
\nabla \times \nabla \times \vec{\psi}_{2}=\omega_{2}^{2} \vec{\psi}_{2} \text { with } \vec{\psi}_{2} \times \hat{n}=0 \text { on } \Gamma
\end{gathered}
$$

The eigenvalues for these two operators are shown in Table 7.18. These eigenvalues agree with the true eigenvalues and with each other. For this particular domain the eigenvalues for the homogeneous Dirichlet and homogeneous Neumann operators agree. The eigenvectors are different but are related by the formula $\vec{\psi}_{2}=\nabla \times \vec{\psi}_{1}$.

Table 7.18: Curl-Curl Eigenvalues with Neumann Boundary Conditions

\begin{tabular}{|c|c||c|c|c|c|}
\hline mode & $\omega_{1,2}^{2}$ true & $\omega_{1}^{2}$ calc & \% error & $\omega_{2}^{2}$ calc & \% error \\
\hline \hline 1 & 19.739209 & 19.940442 & 1.019461 & 19.940442 & 1.019461 \\
\hline 2 & 29.608813 & 29.910664 & 1.019461 & 29.910664 & 1.019461 \\
\hline 3 & 49.348022 & 51.076722 & 3.503078 & 51.076722 & 3.503078 \\
\hline 4 & 59.217626 & 61.046943 & 3.089142 & 61.046943 & 3.089142 \\
\hline 5 & 78.956835 & 82.213002 & 4.123984 & 82.213002 & 4.123984 \\
\hline
\end{tabular}

The dimensions for the operators with the homogeneous Neumann boundary conditions applied are listed in Table 7.19. The dimensions of the range space again agree between the two operators while the null space and total dimensions do not agree due to the different number of degrees of freedom.

Table 7.19: Curl-Curl Discrete Space Dimensions for Neumann Boundary Conditions

\begin{tabular}{|c||c|c|}
\hline Space & 1-form Curl-Curl & 2-form Curl-Curl \\
\hline \hline Dim(Range) & 1701 & 1701 \\
\hline Dim(Null) & 999 & 729 \\
\hline Dim(Domain) & 2700 & 2430 \\
\hline
\end{tabular}




\subsubsection{Spherical cavity}

The electric field in a spherical cavity of radius a can be decomposed into two independent modes: the transverse electric $T E_{r}$ and transverse magnetic $T M_{r}$ modes. The full electric field in the cavity is the sum of these modes with suitable time oscillation terms. These modes can be found in many electrodynamics texts e.g. [30]. The $T E_{r}$ spherical cavity modes are shown in (7.18).

$$
\begin{gathered}
\vec{E}_{r}(r, \theta, \phi)=0 \\
\vec{E}_{\theta}(r, \theta, \phi)=-A_{m n p} \frac{m}{\epsilon r} \frac{1}{r \sin (\theta)} \hat{J}_{n}(\beta r) P_{n}^{m}(\cos (\theta))[-C \sin (m \phi)+D \cos (m \phi)] \\
\vec{E}_{\phi}(r, \theta, \phi)=A_{m n p} \frac{1}{\epsilon r} \hat{J}_{n}^{\prime}(\beta r) P_{n}^{\prime m}(\cos (\theta))[C \cos (m \phi)+D \sin (m \phi)] \\
\vec{E}_{\theta}=0 \text { on } \Gamma \\
\vec{E}_{\phi}=0 \text { on } \Gamma
\end{gathered}
$$

The frequencies (7.19) for the cavity are the roots $\beta=\frac{\gamma_{n, p}}{a}$ of the spherical Bessel functions $j_{n}(\beta r)$.

$$
\begin{aligned}
f & =\frac{1}{2 \pi a \sqrt{\mu \epsilon}} \frac{\gamma_{n, p}}{a} \\
m & =0,1,2,3, \ldots, \leq n \\
n & =1,2,3, \ldots \\
p & =1,2,3, \ldots
\end{aligned}
$$

The $T M_{r}$ cylindrical cavity mode is shown in (7.20).

$$
\begin{aligned}
\vec{E}_{r}(r, \theta, \phi) & =B_{m n p} \frac{\beta^{2}}{j \omega \mu \epsilon} \hat{J}_{n}^{\prime \prime}(\beta r) P_{n}^{m}(\cos (\theta))[C \cos (m \phi)+D \sin (m \phi)] \\
\vec{E}_{\theta}(r, \theta, \phi) & =B_{m n p} \frac{\beta}{i \omega \mu \epsilon r} \hat{J}_{n}^{\prime}(\beta r) P_{n}^{\prime m}(\cos (\theta))[C \phi(m \theta)+D \sin (m \phi)]
\end{aligned}
$$




$$
\begin{gathered}
\vec{E}_{\phi}(r, \theta, \phi)=B_{m n p} \frac{m \beta}{i \omega \mu \epsilon} \frac{1}{r \sin (\theta)} \hat{J}_{n}^{\prime}(\beta r) P_{n}^{m}(\cos (\theta))[-C \sin (m \phi)+D \cos (m \phi)] \\
\vec{E}_{\theta}=0 \text { on } \Gamma \\
\vec{E}_{\phi}=0 \text { on } \Gamma
\end{gathered}
$$

The frequencies (7.20) for the cavity are the $\operatorname{roots} \beta=\frac{\gamma_{n, p}^{\prime}}{a}$ of the the derivative of the spherical Bessel function of order $\mathrm{n} J_{n}^{\prime}(\beta r)$.

$$
\begin{aligned}
f_{m, n, p} & =\frac{1}{2 \pi a \sqrt{\mu \epsilon}} \frac{\gamma_{n, p}^{\prime}}{a} \\
m & =0,1,2,3, \ldots, \leq n \\
n & =1,2,3, \ldots \\
p & =1,2,3, \ldots
\end{aligned}
$$

In this section both the 1-form discrete electric field equation (3.118) and the 2form discrete magnetic flux density (3.115) equations are used to simulate the fields in the spherical cavity with $\epsilon=\mu=1$. The spherical cavity of radius $a=0.05855 \mathrm{~m}$ is exited by the second derivative of a Gaussian over $5 \%$ of the degrees of freedom. The simulations were run for 50 periods of the lowest frequency the $T M_{11}$ mode shown in Table 7.20. The length of the simulation was different in each of the four cases for the hexahedral and tetrahedral grids due to stability constraints.

Table 7.20: Exact Lowest Electrodynamic Frequencies

\begin{tabular}{|c|c|c|c|c|c|}
\hline$T M_{11}$ & $T M_{21}$ & $T E_{11}$ & $T M_{31}$ & $T E_{21}$ & $T M_{41}$ \\
\hline \hline 7.4589 & 10.5655 & 12.2132 & 13.518 & 15.6654 & 16.4782 \\
\hline
\end{tabular}

Ten degrees of freedom were chosen at random and output at every timestep. The time series for each degree of freedom was zero extended to a power of 2 then 
Fourier transformed to determine the power spectrum. The power spectra for the four hexahedral grids listed in Table 7.2 are shown in Figure 7.7. The true frequencies are represented as straight lines. It is apparent that as the grids are refined the peaks approach the true value.
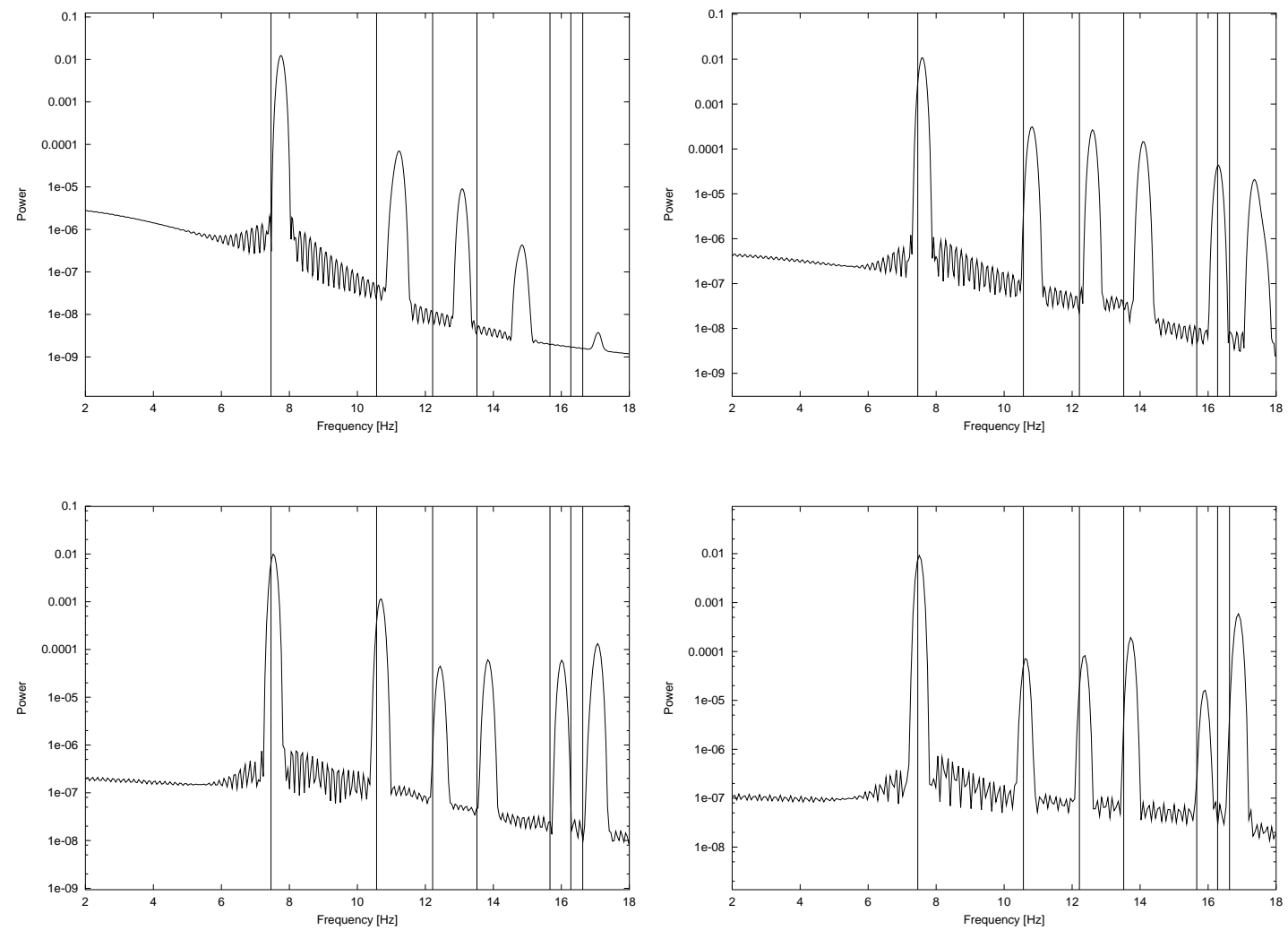

Figure 7.7: Power Spectra for the 1-form electrodynamic simulation for the series of hexahedral grids $\left(\frac{h}{a}=\frac{1}{4}, \frac{1}{6}, \frac{1}{8}, \frac{1}{10}\right.$; left to right,top to bottom)

The power spectra for the four tetrahedral grids listed in Table 7.3 are shown in Figure 7.8. The tetrahedral grids have more edge and face degrees of freedom than an equivalent hexahedral grid with the same number of nodes. The tetrahedral grid results are therefore more accurate than the hexahedral grid results. It is not possible to discretize the spherical domain with prisms therfore only the hexahedral and tetrahedral grid results are presented. 

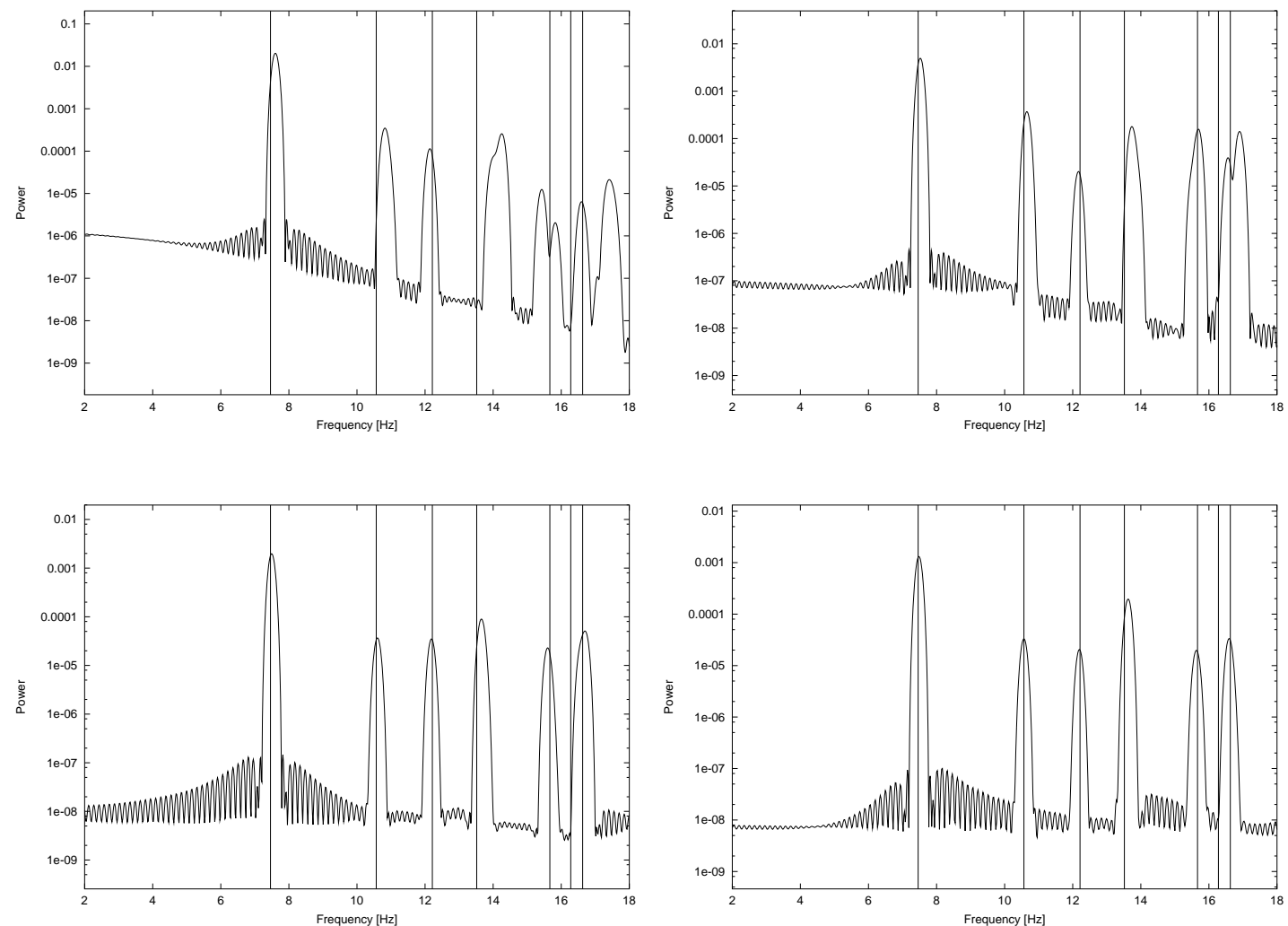

Figure 7.8: Power Spectra for the 1-form electrodynamic simulation for the series of tetrahedral grids $\left(\frac{h}{a}=\frac{1}{4}, \frac{1}{6}, \frac{1}{8}, \frac{1}{10} ;\right.$ left to right,top to bottom) 
A table of the edge length and absolute error form the 1-form electric field equation is shown in Table 7.21. If the logarithm of the error versus the logarithm of $\frac{h}{a}$ is plotted, a least square fit can be calculated. The slope of the resulting fit determines

Table 7.21: 1-form Electrodynamic Refinement Results

\begin{tabular}{|c|c|c|c|c|c|c|}
\hline Element & Parameter & Mesh 1 & Mesh 2 & Mesh 3 & Mesh 4 & Slope \\
\hline \hline Hex & Edge Length & 0.025 & 0.017 & 0.013 & 0.010 & 2.29 \\
\hline & Error & 0.300 & 0.148 & 0.063 & 0.042 & \\
\hline Tet & Edge Length & 0.034 & 0.025 & 0.020 & 0.017 & 2.29 \\
\hline & Error & 0.148 & 0.066 & 0.032 & 0.020 & \\
\hline
\end{tabular}

the accuracy of the method. In this case both the tetrahedral and hexahedral results have a slope of 2.29 showing the method is second order accurate. A plot of the logarithm of the error versus the logarithm of $\frac{h}{a}$ is shown in Figure 7.9.

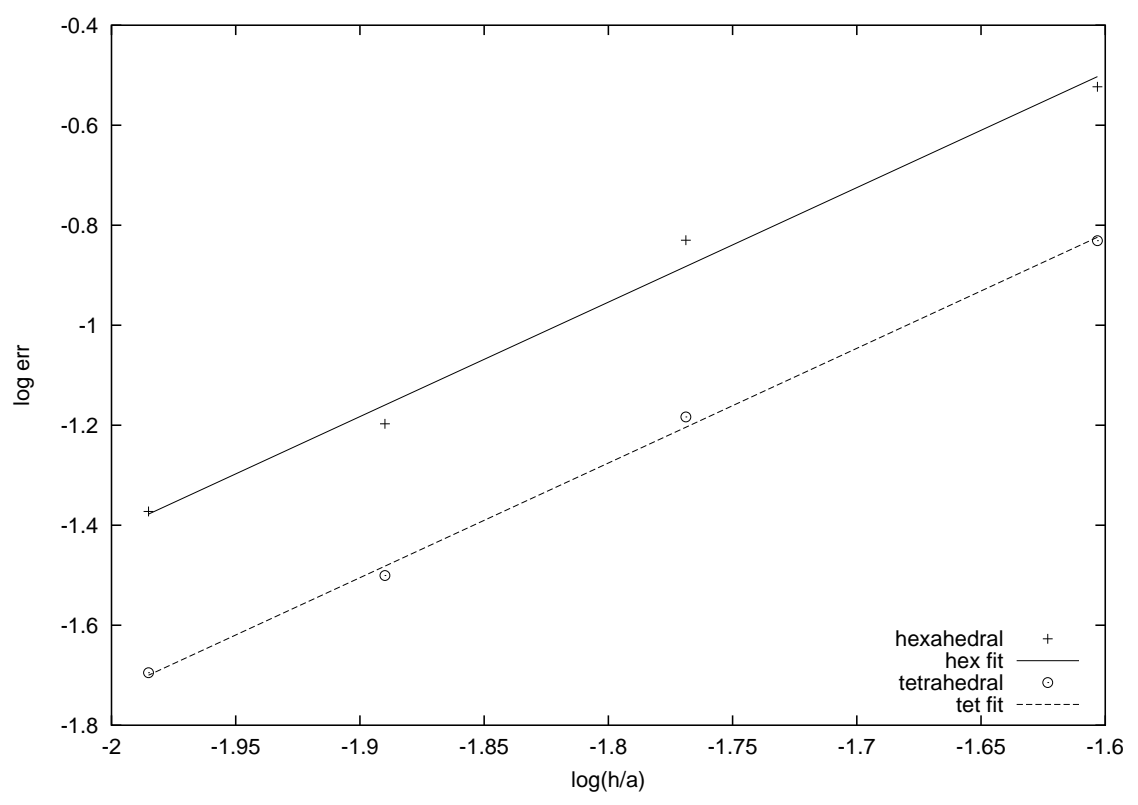

Figure 7.9: Mesh Refinement Results for 1-form Electric Field Wave Equation

The simulation for the 2-form magnetic flux density equation is simulated in the same manner as the 1-form electric field simulation above. The same number of 
degrees of freedom were chosen to excite with the second derivative of a Gaussian. Ten degrees of freedom were chosen to sample for the power spectrum calculations.

The hexahedral grid results for the 2-form magnetic field wave equation are shown in Figure 7.10. These spectra also show the true frequencies as straight lines on the plot.
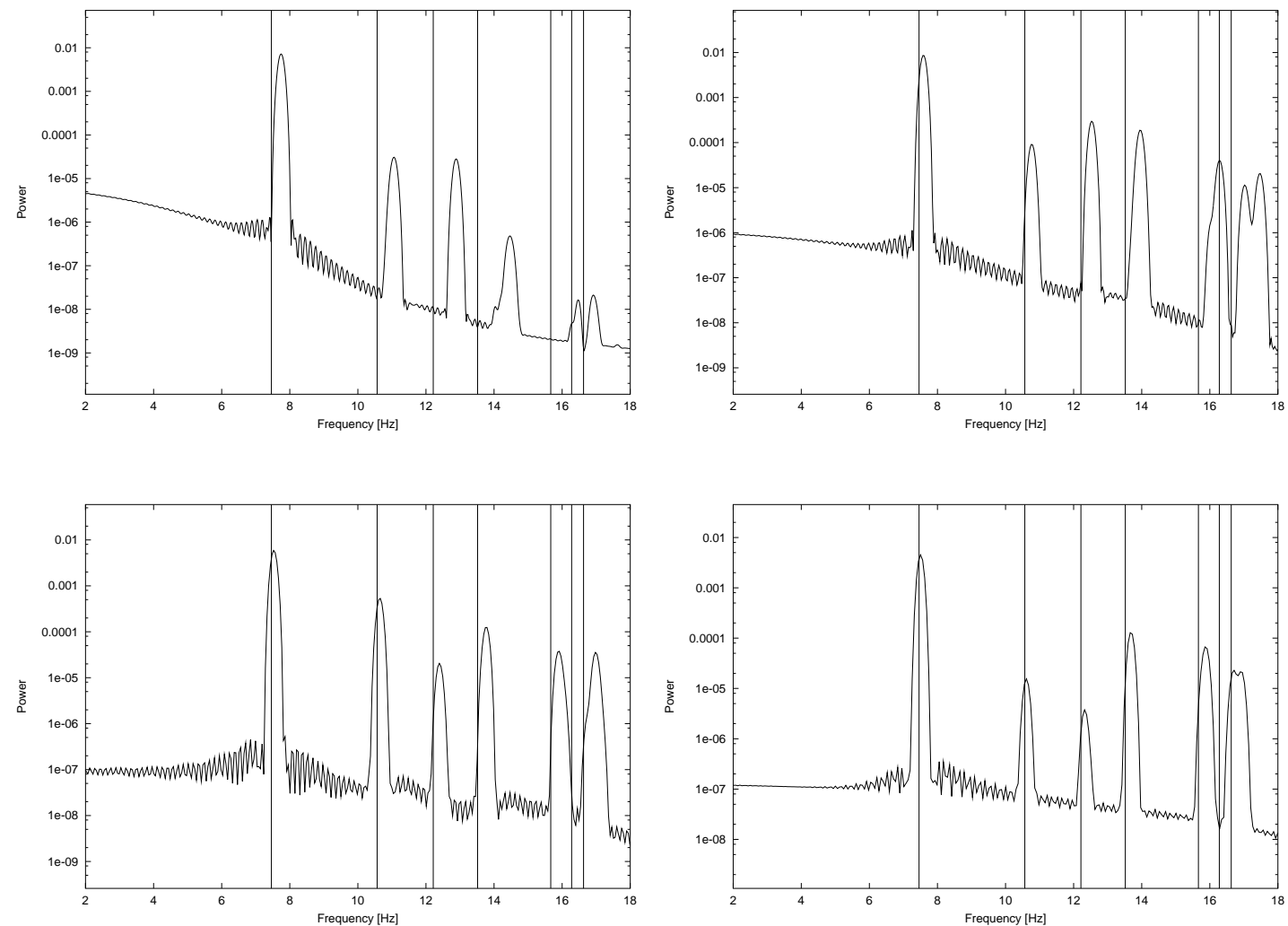

Figure 7.10: Power Spectra for the 2-form electrodynamic simulation for the series of hexahedral grids $\left(\frac{h}{a}=\frac{1}{4}, \frac{1}{6}, \frac{1}{8}, \frac{1}{10} ;\right.$ left to right,top to bottom)

The tetrahedral results for the 2-form magnetic flux density equation are shown in Figure 7.11.

The errors for the hexahedral and tetrahedral spectra are listed in Table 7.22. The tetrahedral results are again better than the hexahedral results due to a larger number of degrees of freedom for the same value of $\frac{h}{a}$. The least square fit of the data 

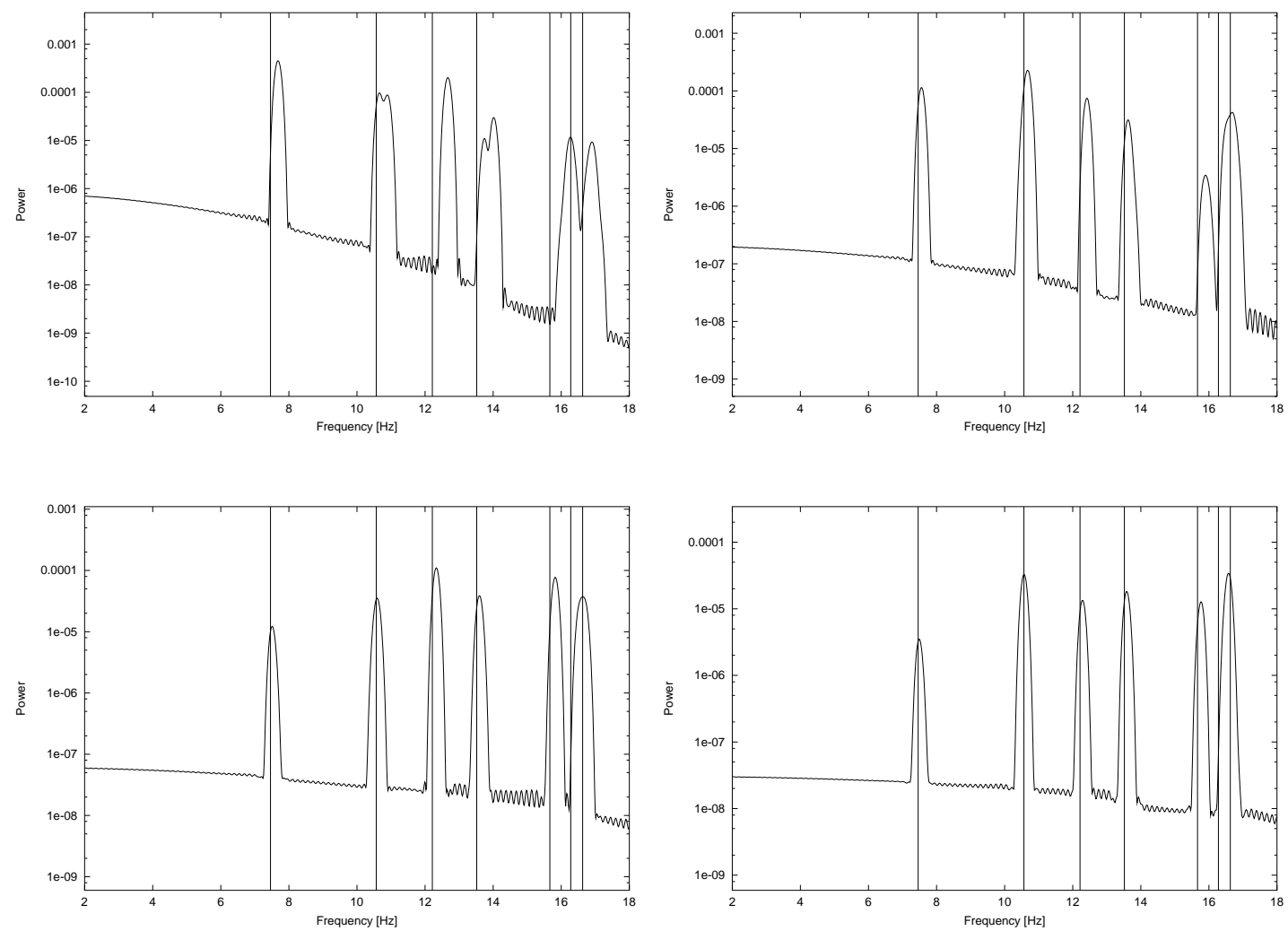

Figure 7.11: Power Spectra for the 2-form electrodynamic simulation for the series of tetrahedral grids $\left(\frac{h}{a}=\frac{1}{4}, \frac{1}{6}, \frac{1}{8}, \frac{1}{10} ;\right.$ left to right,top to bottom) 
shown in Figure 7.12 is 2.14 for the hexahedral case and 2.59 for the tetrahedral case showing the method is second order accurate.

Table 7.22: 2-form Electrodynamic Refinement Results

\begin{tabular}{|c|c|c|c|c|c|c|}
\hline Element & Parameter & Mesh 1 & Mesh 2 & Mesh 3 & Mesh 4 & Slope \\
\hline \hline Hex & Edge Length & 0.025 & 0.017 & 0.013 & 0.010 & 2.14 \\
\hline & Error & 0.287 & 0.115 & 0.065 & 0.044 & \\
\hline Tet & Edge Length & 0.034 & 0.025 & 0.020 & 0.017 & 2.59 \\
\hline & Error & 0.219 & 0.093 & 0.048 & 0.021 & \\
\hline
\end{tabular}

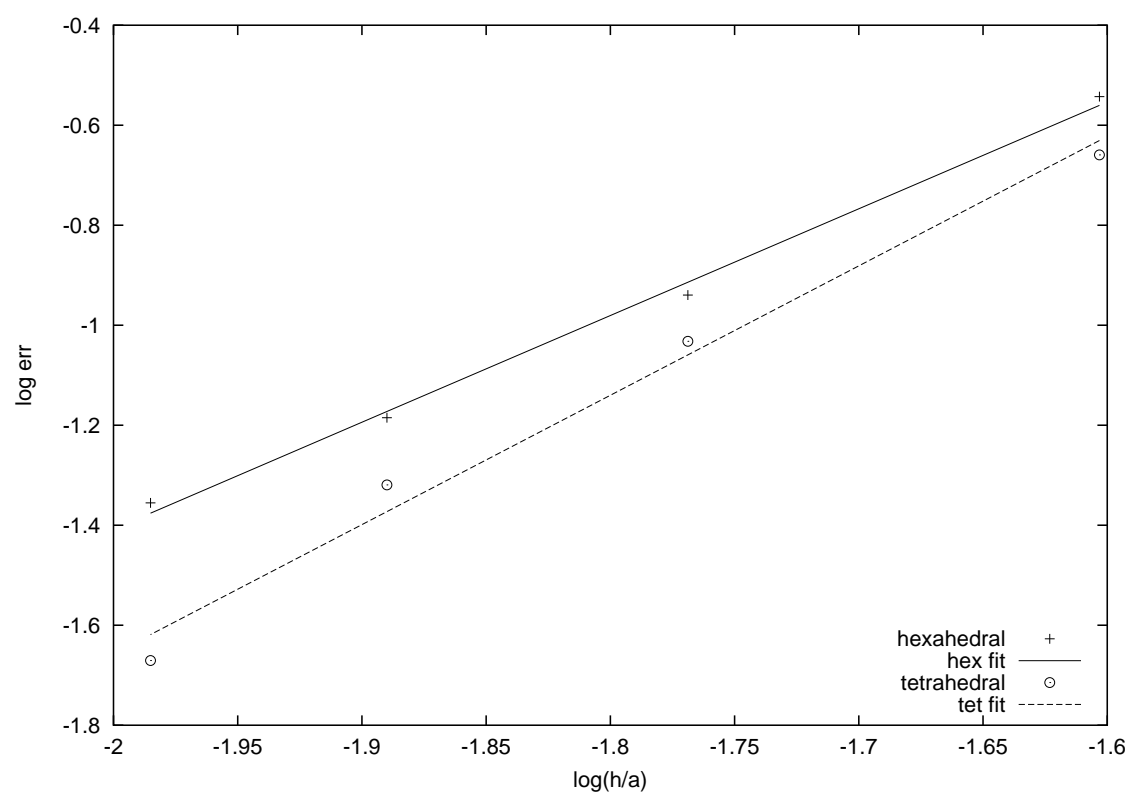

Figure 7.12: Mesh Refinement Results for 2-form Magnetic Field Wave Equation

\subsection{Acoustic Vector Wave Equation}

\subsubsection{Space characterization}

As in the scalar acoustic and electrodynamics equation sections above the eigenvalues and space dimensions will be analyzed for the vector acoustic wave equation. In 
this section the operator being examined is the Grad-Div operator. The eigenvalues for this operator are listed in (7.21).

$$
\omega_{m n l}^{2}=c_{l}^{2} \pi^{2}\left(\frac{m^{2}}{a^{2}}+\frac{n^{2}}{b^{2}}+\frac{l^{2}}{c^{2}}\right)
$$

The first set of eigenvalues is the comparison of the homogeneous Dirichlet boundary conditions of the edge Grad-Div operator and the homogeneous Neumann boundary conditions of the face Grad-Div equation (7.22).

$$
\begin{gathered}
\nabla\left(\nabla \cdot \vec{\psi}_{1}\right)=\omega_{1}^{2} \vec{\psi}_{1} \text { with } \vec{\psi}_{1} \times \hat{n}=0 \text { on } \Gamma \\
\nabla\left(\nabla \cdot \vec{\psi}_{2}\right)=\omega_{2}^{2} \vec{\psi}_{2} \text { with } \vec{\psi}_{2} \cdot \hat{n}=0 \text { on } \Gamma
\end{gathered}
$$

In this case the values for the coefficients $m, n, l$ for the eigenvalues (7.21) are given in $(7.23)$.

$$
\begin{gathered}
m=1,2,3,4 \ldots \\
n=1,2,3,4 \ldots \\
l=1,2,3,4 \ldots
\end{gathered}
$$

The eigenvalues for these two operators are listed in Table 7.23. These eigenvalues agree with the true eigenvalues each other and with the scalar acoustic wave equation values listed in Table 7.9.

The comparison of the space dimensions for these operators are listed in Table 7.24. The dimensions of these operators agree for the null space in contrast with the Curl-Curl operators which agreed in the range space dimensions. Also note that the dimension of these operators null space is the same size as the range spaces of the Curl-Curl operators with Neumann boundary. 
Table 7.23: Grad-Div Eigenvalues with Dirichlet Boundary Conditions

\begin{tabular}{|c|c||c|c|c|c|}
\hline mode & $\omega_{1,2}^{2}$ true & $\omega_{1}^{2}$ calc & \% error & $\omega_{2}^{2}$ calc & \% error \\
\hline \hline 1 & 29.608813 & 29.910665 & 1.019465 & 29.910664 & 1.019461 \\
\hline 2 & 59.217626 & 61.046938 & 3.089133 & 61.046943 & 3.089142 \\
\hline 3 & 88.826440 & 92.183211 & 3.779022 & 92.183223 & 3.779036 \\
\hline 4 & 108.565648 & 117.140453 & 7.898267 & 117.140422 & 7.898239 \\
\hline 5 & 118.435253 & 123.319483 & 4.123967 & 123.319504 & 4.123985 \\
\hline
\end{tabular}

Table 7.24: Grad-Div Discrete Space Dimensions for Dirichlet Boundary Conditions

\begin{tabular}{|c||c|c|}
\hline Space & 1-form Grad-Div & 2-form Curl-Curl \\
\hline \hline Dim(Range) & 512 & 728 \\
\hline Dim(Null) & 1216 & 1216 \\
\hline Dim(Domain) & 1728 & 1944 \\
\hline
\end{tabular}

A comparison of the homogeneous Neumann boundary conditions of the edge Grad-Div operator and the homogeneous Dirichlet boundary conditions of the face Grad-Div equation (7.22) are shown in Table 7.25.

$$
\begin{array}{r}
\nabla\left(\nabla \cdot \vec{\psi}_{1}\right)=\omega_{1}^{2} \vec{\psi}_{1} \text { with } \nabla \times \vec{\psi}_{1} \times \hat{n}=0 \text { on } \Gamma \\
\nabla\left(\nabla \cdot \vec{\psi}_{2}\right)=\omega_{2}^{2} \vec{\psi}_{2} \text { with } \nabla \cdot \vec{\psi}_{2}=0 \text { on } \Gamma
\end{array}
$$

In this case the values for the coefficients $m, n, l$ for the eigenvalues (7.21) are given in $(7.25)$.

$$
\begin{gathered}
m=0,1,2,3,4 \ldots \\
n=0,1,2,3,4 \ldots \\
l=0,1,2,3,4 \ldots
\end{gathered}
$$

These eigenvalues are the same as the scalar eigenvalues listed in Table 7.11.

The space dimensions for these operators follow the same pattern as the eigenvalues listed above. The null space dimensions agree while the range and total space 
Table 7.25: Grad-Div Eigenvalues with Neumann Boundary Conditions

\begin{tabular}{|c|c||c|c|c|c|}
\hline mode & $\omega_{1,2}^{2}$ true & $\omega_{1}^{2}$ calc & \% error & $\omega_{2}^{2}$ calc & \% error \\
\hline \hline 1 & 9.869604 & 9.970221 & 1.019461 & 9.970222 & 1.019465 \\
\hline 2 & 19.739209 & 19.940442 & 1.019461 & 19.940443 & 1.019465 \\
\hline 3 & 29.608813 & 29.910664 & 1.019461 & 29.910665 & 1.019465 \\
\hline 4 & 39.478418 & 41.106501 & 4.123984 & 41.106495 & 4.123967 \\
\hline 5 & 49.348022 & 51.076722 & 3.503080 & 51.076716 & 3.503067 \\
\hline
\end{tabular}

dimensions differ. The dimension of the null space for these Grad-Div operators are also the same as the dimensions of the range space for the Curl-Curl operators with Dirichlet boundary conditions.

Table 7.26: Grad-Div Discrete Space Dimensions for Neumann Boundary Conditions

\begin{tabular}{|c||c|c|}
\hline Space & 1-form Grad-Div & 2-form Grad-Div \\
\hline \hline Dim(Range) & 999 & 729 \\
\hline Dim(Null) & 1701 & 1701 \\
\hline Dim(Domain) & 2700 & 2430 \\
\hline
\end{tabular}

\subsubsection{Spherical cavity}

The velocity field in a spherical cavity of radius $a$ can be derived from the scalar wave equations spherical modes using the mode as the velocity potential $\phi$. The velocity and the velocity potential are related through the formula $\vec{V}=-\nabla \phi$. The velocity modes for the spherical cavity are shown in (7.26).

$$
\begin{aligned}
\vec{V}_{r}(r, \theta, \phi) & =-A_{m n p} \beta J_{n}^{\prime}(\beta r) P_{n}^{m}(\cos (\theta))[B \cos (m \phi)+C \sin (m \phi)] \\
\vec{V}_{\theta}(r, \theta, \phi) & =-A_{m n p} \frac{1}{r} J_{n}(\beta r) P_{n}^{\prime m}(\cos (\theta))[B \cos (m \phi)+C \sin (m \phi)] \\
\vec{V}_{\phi}(r, \theta, z) & =-A_{m n p} \frac{m}{r \sin (\theta)} J_{n}(\beta r) P_{n}^{m}(\cos (\theta))[-B \sin (m \phi)+C \cos (m \phi)] \\
\vec{V} \cdot \hat{n} & =0 \text { on } \Gamma
\end{aligned}
$$


The frequencies (7.27) for the cavity are a combination of the roots $\beta=\frac{\pi \alpha_{n, p}}{a}$ of the derivative of the spherical Bessel functions $\hat{J}_{n}^{\prime}(\beta r)$. The exact frequencies are the same values as in the scalar acoustic wave equation Table 7.13.

$$
\begin{aligned}
f & =\frac{c_{l}}{2} \frac{\alpha_{n, p}}{a} \\
m & =0,1,2,3, \ldots \\
n & =0,1,2,3, \ldots \\
p & =1,2,3, \ldots
\end{aligned}
$$

The vector acoustic wave equation can be simulated using both a 1-form discretization and a 2-form discretization just as either form can be used in the electrodynamic simulations. The second order 1 -form acoustic wave equation (3.127) with $c_{l}=1$ is used to produce the power spectra for the hexahedral grids listed in Table 7.2 and are shown in Figure 7.13. As in the previous spherical cavity simulations $5 \%$ of the total degrees of freedom are chosen to excite with the second derivative of a Gaussian. Ten output degrees of freedom are chosen to calculate the power spectra as in the previous spherical cavity sections.

The corresponding tetrahedral grid power spectra for the grids listed in Table 7.3 are shown in Figure 7.14. As in the previous cases the tetrahedral results are more accurate due to the larger number of 1-form degrees of freedom.

The errors and edge lengths for the hexahedral 1-form acoustic wave equation simulations are listed in Table 7.27. If a least squares fit is applied to the plots of the logarithm of error versus the logarithm of $\frac{h}{a}$, Figure 7.15 , slopes of 1.83 and 1.95 for the hexahedral and tetrahedral grids respectively result. These slopes show 

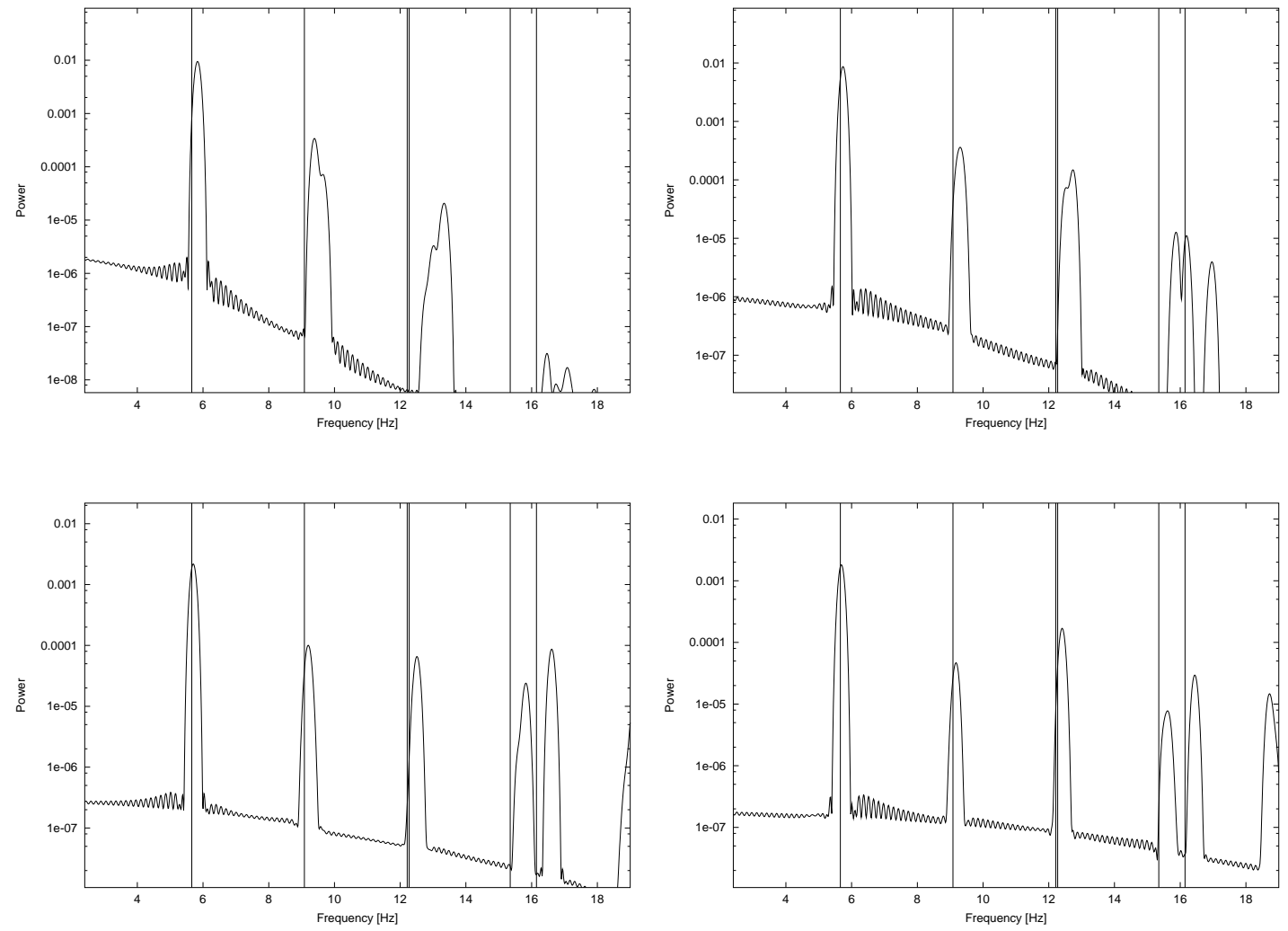

Figure 7.13: Power Spectra for the 1-form acoustic simulation for the series of hexahedral grids $\left(\frac{h}{a}=\frac{1}{4}, \frac{1}{6}, \frac{1}{8}, \frac{1}{10}\right.$; left to right,top to bottom) 

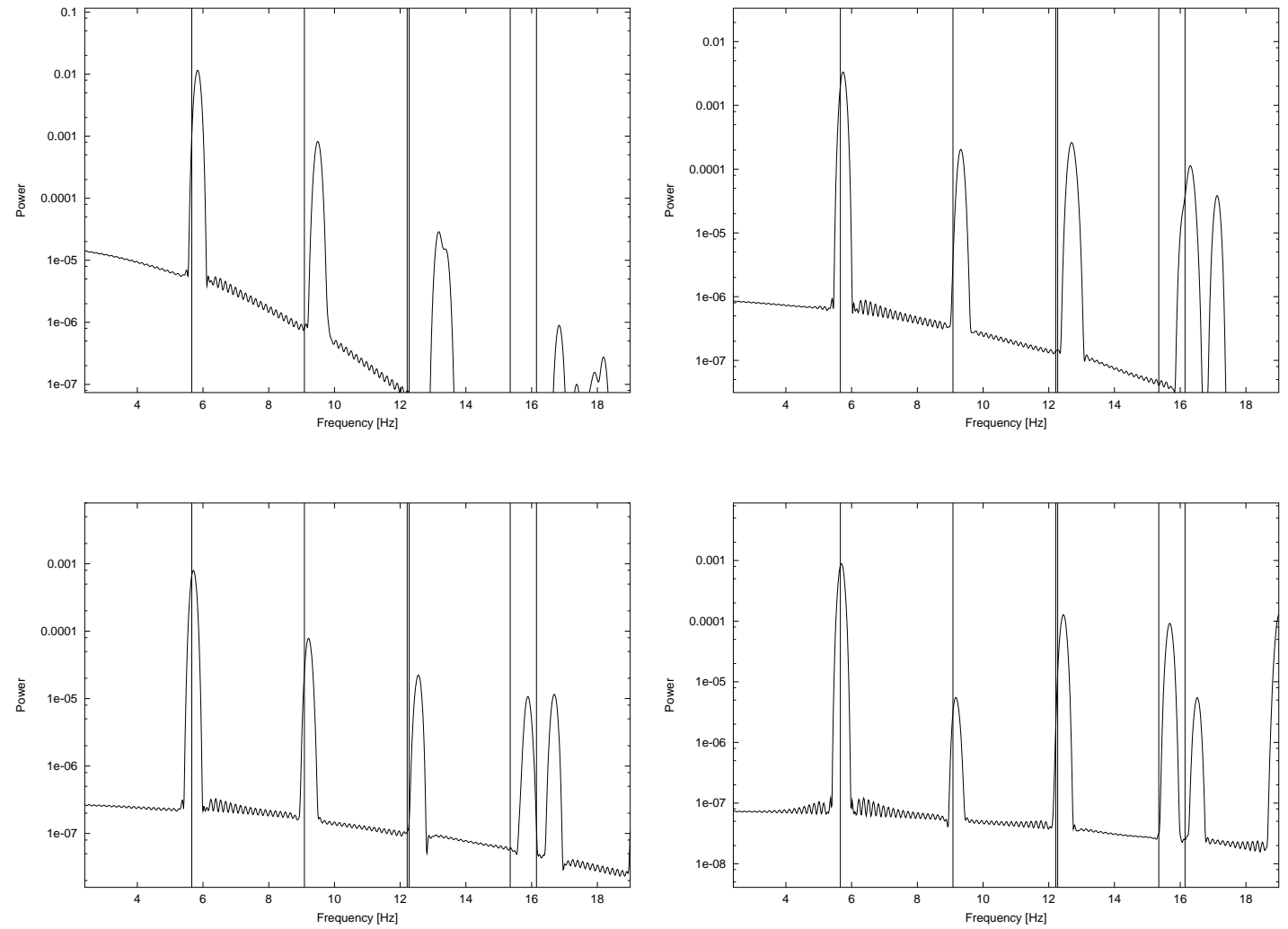

Figure 7.14: Power Spectra for the 1-form acoustic simulation for the series of tetrahedral grids $\left(\frac{h}{a}=\frac{1}{4}, \frac{1}{6}, \frac{1}{8}, \frac{1}{10}\right.$; left to right,top to bottom) 
the 1-form vector acoustic wave equation is second order accurate agreeing with the dispersion relation.

Table 7.27: 1-form Acoustic Refinement Results

\begin{tabular}{|c|c|c|c|c|c|c|}
\hline Element & Parameter & Mesh 1 & Mesh 2 & Mesh 3 & Mesh 4 & Slope \\
\hline \hline Hex & Edge Length & 0.025 & 0.017 & 0.013 & 0.010 & 1.83 \\
\hline & Error & 0.176 & 0.085 & 0.046 & 0.037 & \\
\hline Tet & Edge Length & 0.034 & 0.025 & 0.020 & 0.017 & 1.95 \\
\hline & Error & 0.176 & 0.081 & 0.053 & 0.030 & \\
\hline
\end{tabular}

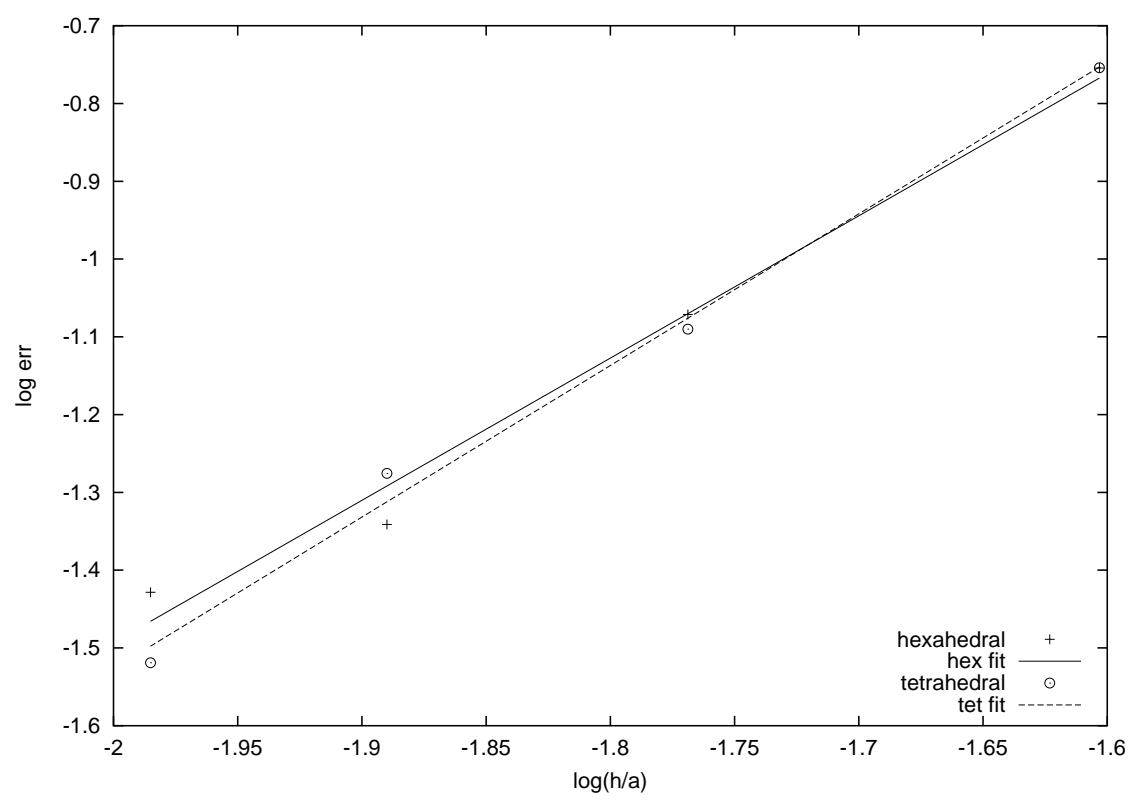

Figure 7.15: Mesh Refinement Results for 1-form Velocity Field Wave Equation

The natural discrete formulation for the vector acoustic wave equation is listed in (3.133). A sound speed of $c_{l}=1$ is used in this 2 -form formulation. The same method as the 1-form simulations was applied to the hexahedral and tetrahedral spherical grids.

The power spectra for the hexahedral grids listed in Table 7.2 are shown in Figure 7.16. The exact frequencies are represented as vertical lines in the plots. 

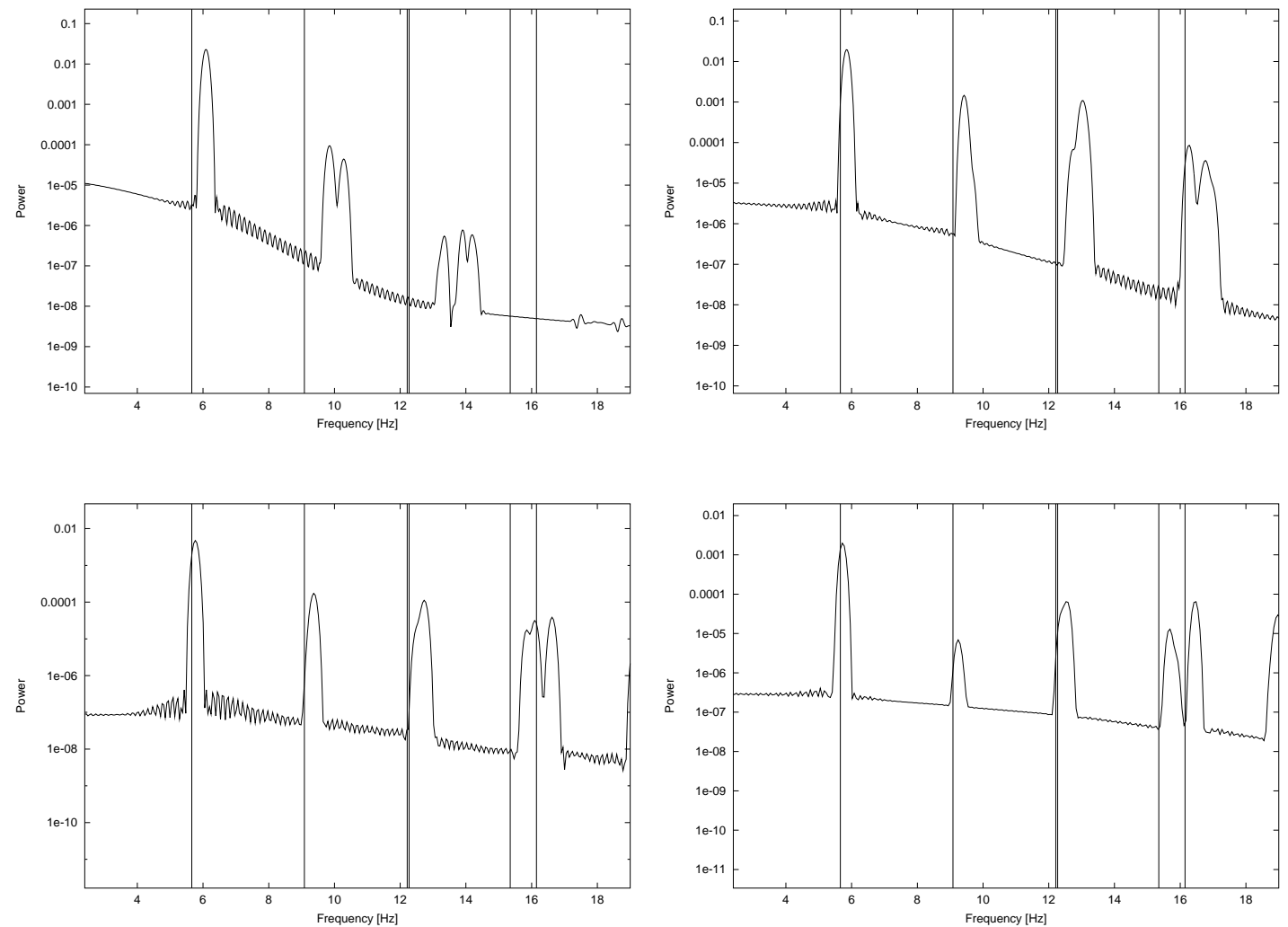

Figure 7.16: Power Spectra for the 2-form acoustic simulation for the series of hexahedral grids $\left(\frac{h}{a}=\frac{1}{4}, \frac{1}{6}, \frac{1}{8}, \frac{1}{10}\right.$; left to right,top to bottom) 
The power spectra for the tetrahedral grids listed in Table 7.3 are shown in Figure 7.17.
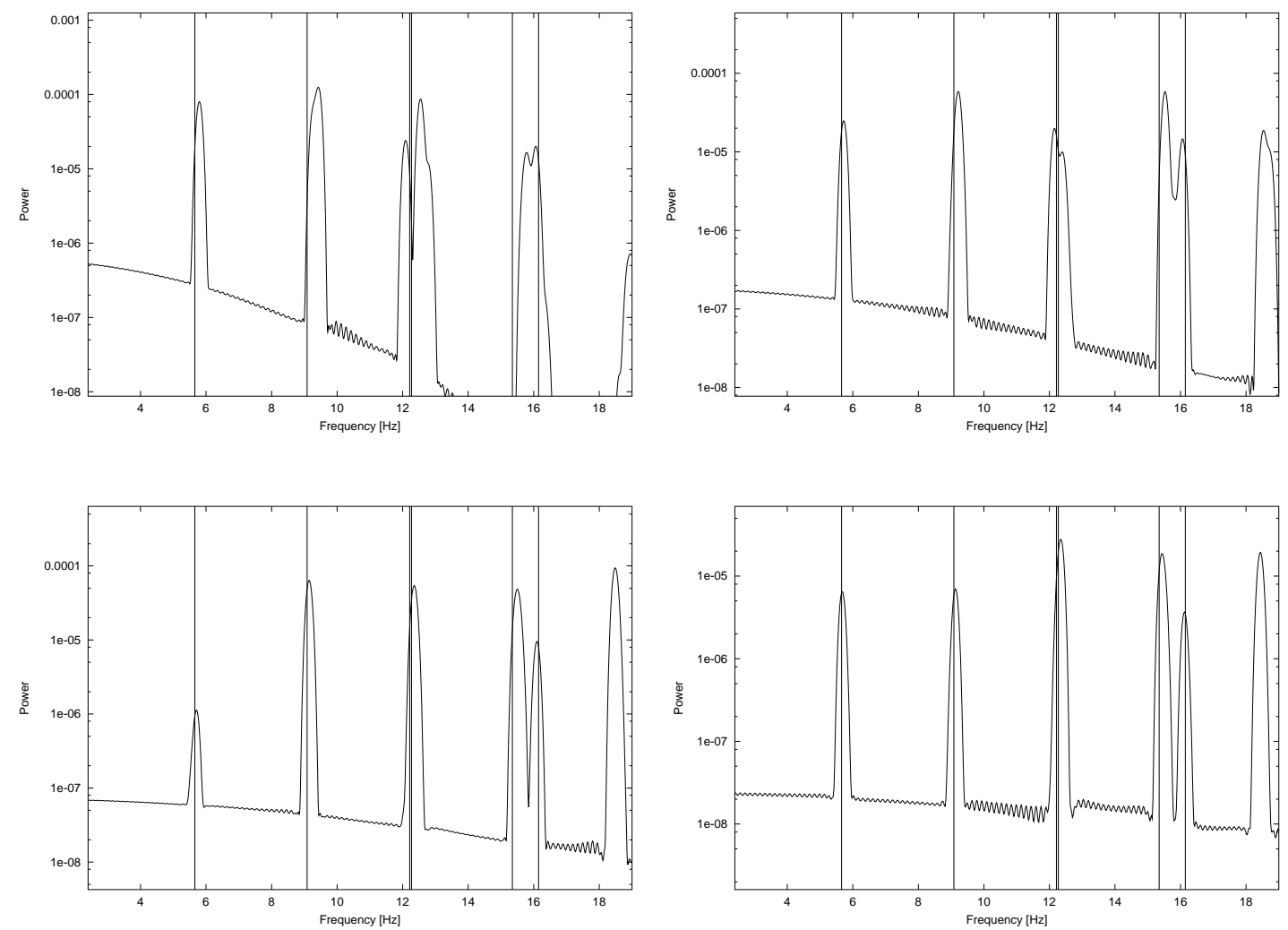

Figure 7.17: Power Spectra for the 2-form acoustic simulation for the series of tetrahedral grids $\left(\frac{h}{a}=\frac{1}{4}, \frac{1}{6}, \frac{1}{8}, \frac{1}{10} ;\right.$ left to right,top to bottom)

The edge lengths and errors are listed in Table 7.28. The plots of the logarithm of the error versus the logarithm of $\frac{h}{a}$ along with the least squares fit are shown in Figure 7.18. The number of degrees of freedom in the tetrahedral grids is larger than the number in the hexahedral grids giving lower errors. The hexahedral grid results give a slope of 2.22 while the tetrahedral grid results show a slope of 2.40. Both slopes agree with the dispersion relation which states this discrete 2-form acoustic wave equation is second order accurate. 
Table 7.28: 2-form Acoustic Refinement Results

\begin{tabular}{|c|c|c|c|c|c|c|}
\hline Element & Parameter & Mesh 1 & Mesh 2 & Mesh 3 & Mesh 4 & Slope \\
\hline \multirow[t]{2}{*}{ Hex } & Edge Length & 0.025 & 0.017 & 0.013 & 0.010 & 2.22 \\
\hline & Error & 0.424 & 0.178 & 0.106 & 0.058 & \\
\hline \multirow[t]{2}{*}{ Tet } & Edge Length & 0.034 & 0.025 & 0.020 & 0.017 & 2.40 \\
\hline & Error & 0.139 & 0.063 & 0.024 & 0.012 & \\
\hline
\end{tabular}

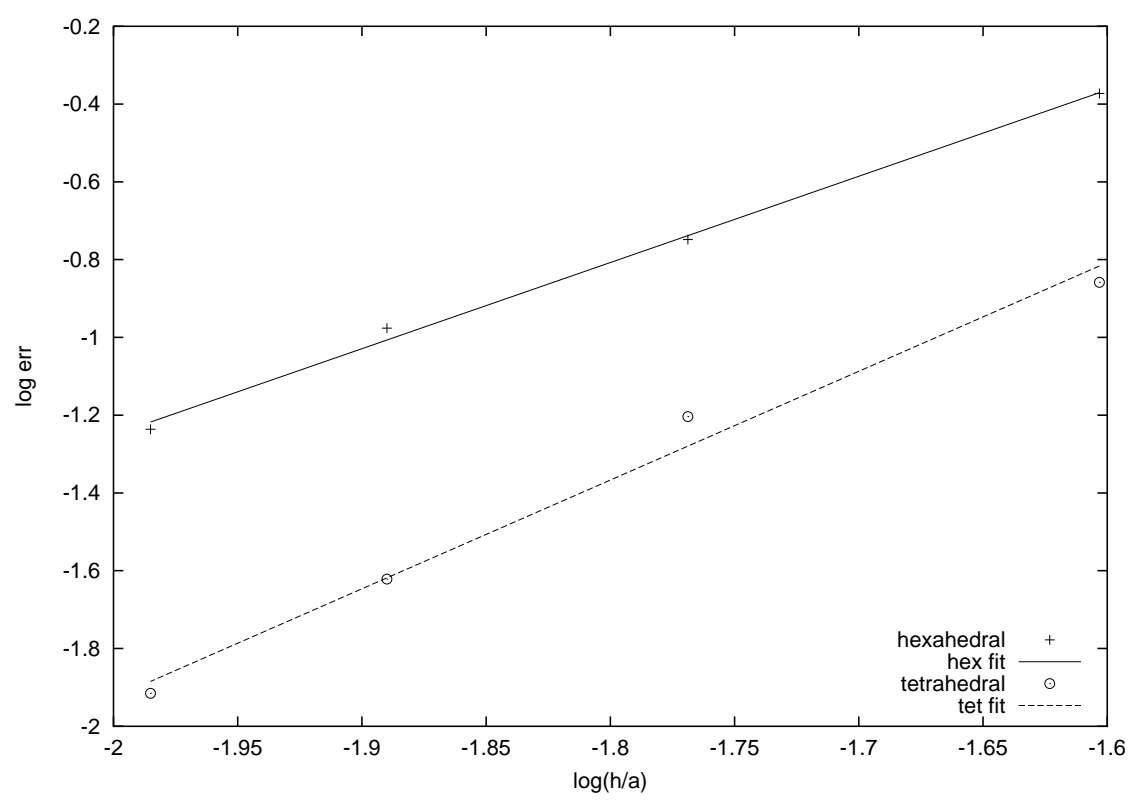

Figure 7.18: Mesh Refinement Results for 2-form Velocity Field Wave Equation 


\subsection{Combined Vector Wave Equations}

In the next two sections preliminary work combining natural and adjoint operators as well as more esoteric operators is researched. The first of these combined wave equations is the elasticity equation. This equation involves combining a natural second order differential operator with the adjoint second order differential operator resulting in the vector Laplacian. The second combined wave equation is the linear magnetohydrodynamic equation. This equation involves the vector acoustic operator with a transverse wave term. Both of the combined wave equations are simulated on hexahedral domains with one element in the $\mathrm{z}$ direction. This allows an effectively two dimensional simulation on a three dimensional mesh.

\subsubsection{Linear elastic waves}

The second order linear elastic wave equation involves the combination of the natural second order differential operator with the adjoint differential operator resulting in the vector Laplacian operator. In the following simulation the displacement $\vec{u}$ will be represented as either a 1-form or a 2-form. The natural and adjoint operators for both of these forms have been presented in the previous sections. Boundary conditions for the linear elastic equation require specification of all three components of the displacement or the traction. Using the differential forms formulation limits the possible boundary conditions to specifying the normal or tangential component of the displacement or specific components of the flux. Each term in the discrete linear elastic equations has a different boundary condition associated with it. Using the Dirichlet boundary conditions for each of the operators constrains all of the 
components on the boundary.

The mesh used in the space characterization sections for the scalar Laplacian Curl-Curl and Grad-Div operators above is used in this section to verify the vector Laplacian operator. The $10 \times 10 \times 10$ element hexahedral grid is used to determine the eigenvalues for the domain with homogeneous Dirichlet boundary conditions. The homogeneous Dirichlet boundary conditions correspond to the natural boundary conditions for the 1-form vector Laplacian and the essential boundary conditions for the 2-form vector Laplacian.

Table 7.29: Vector Laplacian Eigenvalues with Dirichlet Boundary Conditions

\begin{tabular}{|c||c|c|c|c|c|}
\hline mode & $\omega^{2}$ true & $\omega_{1}^{2}$ calc & \% error & $\omega_{2}^{2}$ calc & \% error \\
\hline \hline 1 & 9.869604 & 9.951043 & 0.825 & 9.951043 & 0.825 \\
\hline 2 & 19.739209 & 19.94029 & 1.02 & 19.94029 & 1.02 \\
\hline 3 & 29.608813 & 29.85313 & 0.825 & 29.85313 & 0.825 \\
\hline 4 & 39.478418 & 40.79356 & 1.00 & 40.79356 & 1.00 \\
\hline 5 & 49.348022 & 50.74460 & 2.83 & 50.74463 & 2.83 \\
\hline
\end{tabular}

The simulation represented below analyzes the speeds of the longitudinal and transverse waves to verify the discretization. The parameters for the square hexahedral grid with dimensions $[0,1] \mathrm{x}[0,1]$ used in the simulation are shown in Table 7.30. This grid is effectively two-dimensional with a single cell in the third dimension so that the three-dimensional matrix generator may be utilized. At the center of the grid a displacement source defined by the second derivative of a Gaussian is used. The longitudinal and transverse waves will propagate outward with a circular pattern. The simulation is halted when the longitudinal wave encounters the boundary. Four snapshots of the time series are shown in Figure 7.19. 

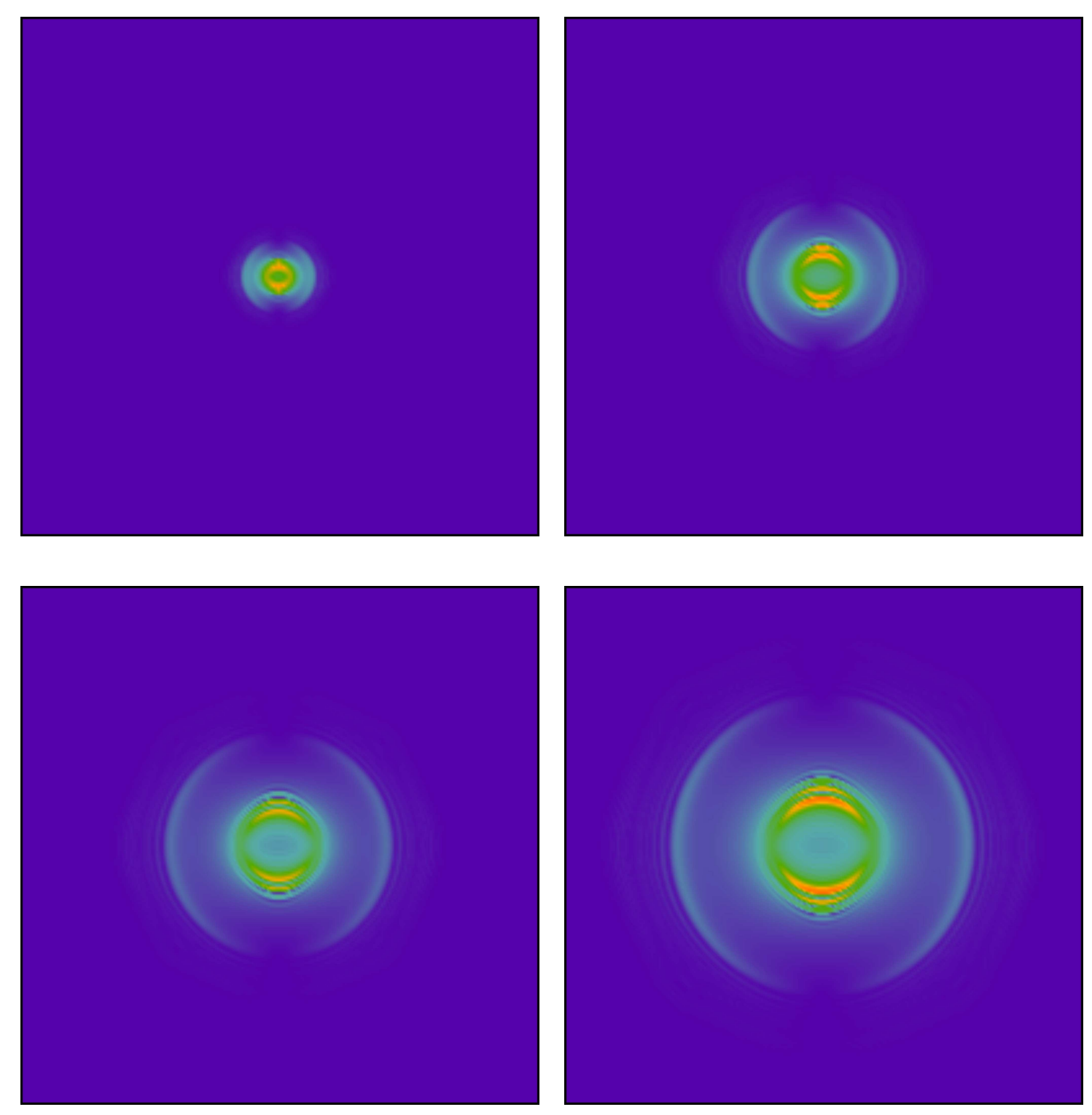

Figure 7.19: 2D 2-form linear elastic wave displacement magnitude

Table 7.30: Hexahedral Elastic Wave Mesh

\begin{tabular}{|c|c|c|c|}
\hline Elements & Nodes & Edges & Faces \\
\hline \hline 39601 & 80000 & 199200 & 158802 \\
\hline
\end{tabular}


Table 7.31: 2-form Elastic wave speeds

\begin{tabular}{|c|c|c|c|}
\hline$c_{l}$ & \% error & $c_{t}$ & \% error \\
\hline \hline 1.29390 & 3.79 & 0.431768 & 0.743 \\
\hline
\end{tabular}

\subsubsection{Linear magnetohydrodynamic modes}

The second order linear magnetohydrodynamics equation consists of an acoustic term and a transverse wave term. In this section the eigenvalues for the transverse wave term will be calculated. The acoustic eigenvalues for a three dimensional rectangular cavity have been computed above and will not be repeated for the two dimensional region used in this section. The mesh used in this section is a 10x10x1 Cartesian mesh with uniform spacing. The one element in the $z$-direction is used to make the mesh logically three dimensional in order to utilize the three dimensional mesh generator. The eigenvalue results for the second order transverse eigenvalue equation (7.28) are given below

$$
\lambda=\frac{\left(n^{2}+m^{2}\right) \pi^{2}}{L_{x}^{2} L_{y}^{2}}
$$

where $L_{x}=1, L_{y}=1, n, m=1,2, \ldots$. The eigenvalues are solved for two different situations. $\vec{v} \cdot \hat{n}=0$ on $\Gamma, \vec{v} \cdot \hat{n}=0$ on $\Gamma_{x}$ and $\Gamma_{z}$ and $\vec{v} \cdot \hat{t}=0$ on $\Gamma_{y}$

Table 7.32: MHD term eigenvalues

\begin{tabular}{|c|c||c|c|c|}
\hline $\mathrm{m}$ & $\mathrm{n}$ & Calculated & Exact & Rel. Error \\
\hline \hline 1 & 1 & 19.7371 & 19.7392 & 0.0107 \\
\hline 1 & 2 & 49.0196 & 49.3480 & 0.6655 \\
\hline 1 & 2 & 50.3238 & 49.3480 & 1.973 \\
\hline 2 & 2 & 78.8058 & 78.9568 & 0.19 \\
\hline 2 & 2 & 78.8136 & 78.9568 & 0.18 \\
\hline
\end{tabular}


Table 7.33: MHD term eigenvalues

\begin{tabular}{|c|c||c|c|c|}
\hline $\mathrm{m}$ & $\mathrm{n}$ & Calculated & Exact & Rel. Error \\
\hline \hline 1 & 1 & 19.7371 & 19.7392 & 0.0107 \\
\hline 1 & 2 & 49.0196 & 49.3480 & 0.6655 \\
\hline 1 & 2 & 50.3238 & 49.3480 & 1.973 \\
\hline 2 & 2 & 78.8058 & 78.9568 & 0.19 \\
\hline 2 & 2 & 78.8136 & 78.9568 & 0.18 \\
\hline
\end{tabular}
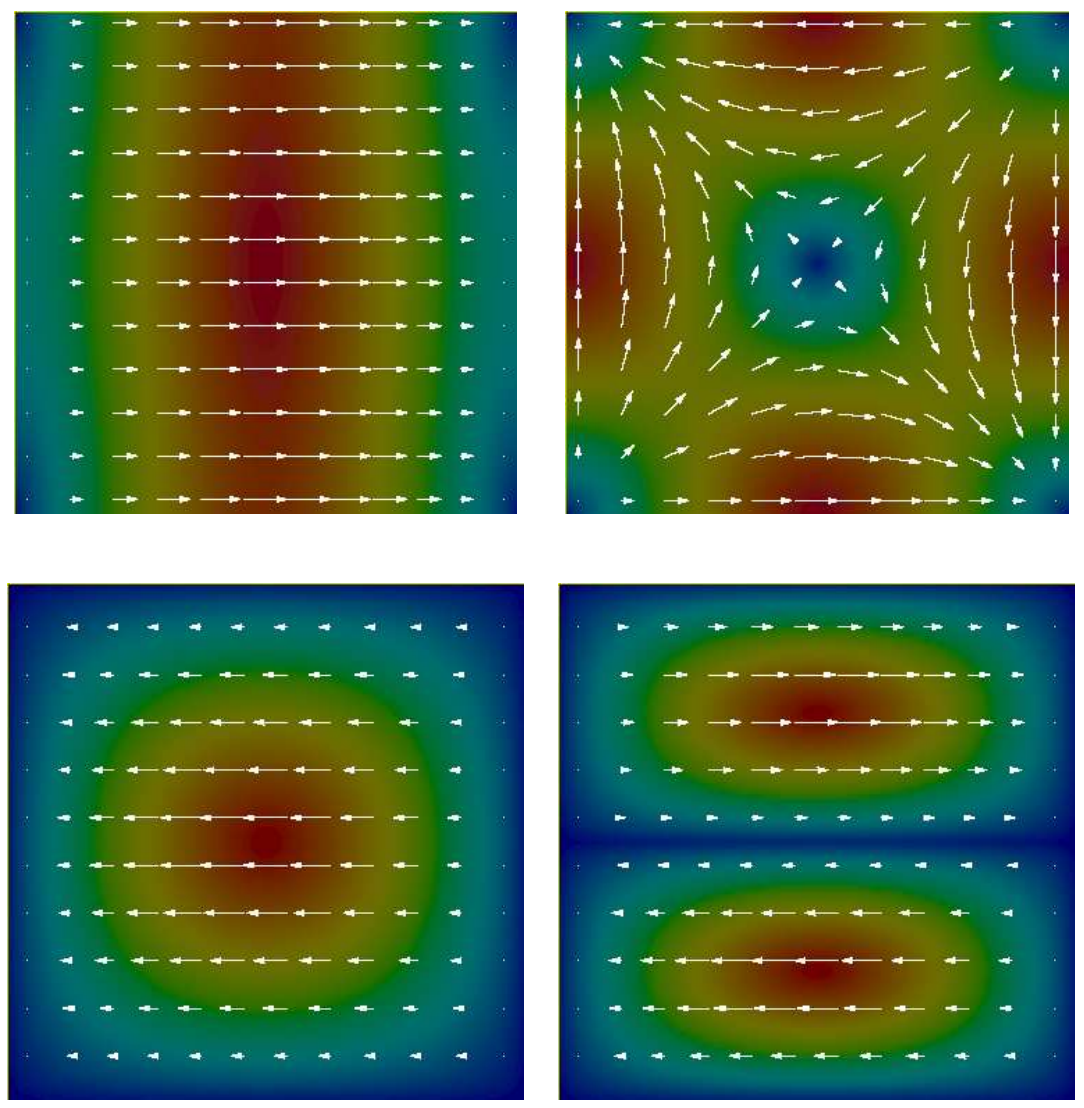

Figure 7.20: 2 Dimensional Acoustic and MHD Term Eigenmodes 


\subsection{Parallel Results}

As stated in the introduction of this dissertation the main focus is simulation of large electro-optics devices. These simulations require small timesteps for stability and long run times for analysis. They also require large amounts of random access memory (RAM) to store the system matrices. All of these requirements lead to the necessity of running the problems in parallel. The parallel method used is based on the Message Passing Interface (MPI) discussed in Chapter 6. Also discussed Chapter 6 were the limitations of the parallel method with respect to communication overhead versus overall efficiency. If the parallelism does not decrease the runtime for a fixed problem size then the utility of the formulation is suspect.

This section applies several metrics to a larger version of the cylindrical grid used in the previous sections. The grid consists of 100,000 hexahedrons with 304,000 faces, 308,090 edges and 104,091 nodes. The four equations in scalar and vector acoustic equations as well as the two electrodynamics equations are analyzed. The formulations will be analyzed for parallel runs with $2^{p}$ number of processors with $p=0,1,2,3,4,5$.

The results are shown in Table 7.34. The metrics used are the number of Jacobi preconditioned Conjugate Gradient method iterations per solve, the total time for a single run, the time for building the matrix without communication, and the time for communicating the off processor data. All of the simulations were run for the same total simulation time.

As discussed previously in Section 4.3 the number of Jacobi preconditioned Conjugate Gradient method iterations is independent of the number of processors used. 
Table 7.34: Hexahedral Grid Parallel Results

\begin{tabular}{|c|c|c|c|c|c|c|c|}
\hline Form & Metric & 1 & 2 & 4 & 8 & 16 & 32 \\
\hline \multirow{4}{*}{0} & JCG Iter. & $\overline{21}$ & $\overline{21}$ & 21 & 21 & $\overline{221}$ & 21 \\
\hline & $\begin{array}{c}\text { JCG } \\
\text { CPU Secs. }\end{array}$ & 6586 & 2479 & 1359 & 687 & 382 & 213 \\
\hline & $\begin{array}{c}\text { Matrix } \\
\text { Build Secs. }\end{array}$ & 103.71 & 25.44 & 12.26 & 6.18 & 3.29 & 1.81 \\
\hline & $\begin{array}{c}\text { Matrix } \\
\text { Assembly Secs. }\end{array}$ & 0.00 & 13.70 & 3.74 & 3.25 & 2.11 & 1.75 \\
\hline \multirow{4}{*}{1} & JCG Iter. & 17 & 17 & 17 & 17 & 17 & 17 \\
\hline & $\begin{array}{c}\text { JCG } \\
\text { CPU Secs. }\end{array}$ & 3640 & 2609 & 1393 & 725 & 390 & 212 \\
\hline & $\begin{array}{c}\text { Matrix } \\
\text { Build Secs. }\end{array}$ & 183.57 & 66.21 & 32.56 & 11.01 & 5.68 & 2.92 \\
\hline & $\begin{array}{c}\text { Matrix } \\
\text { Assembly Secs. }\end{array}$ & 0.00 & 16.14 & 11.31 & 7.61 & 4.97 & 3.86 \\
\hline \multirow{4}{*}{2} & JCG Iter. & 17 & 17 & 17 & 17 & 17 & 17 \\
\hline & $\begin{array}{c}\text { JCG } \\
\text { CPU Secs. }\end{array}$ & 3456 & 3052 & 1605 & 923 & 443 & 246 \\
\hline & $\begin{array}{c}\text { Matrix } \\
\text { Build Secs. }\end{array}$ & 88.67 & 63.22 & 20.64 & 10.67 & 5.52 & 2.82 \\
\hline & $\begin{array}{c}\text { Matrix } \\
\text { Assembly Secs }\end{array}$ & 0.00 & 18.21 & 9.39 & 9.33 & 5.87 & 4.58 \\
\hline \multirow{4}{*}{3} & JCG Iter. & 17 & 17 & 17 & 17 & 17 & 17 \\
\hline & $\begin{array}{c}\text { JCG } \\
\text { CPU Secs. }\end{array}$ & 3062 & 2659 & 1382 & 799 & 394 & 235 \\
\hline & $\begin{array}{c}\text { Matrix } \\
\text { Build Secs. }\end{array}$ & 34.92 & 25.82 & 18.62 & 4.36 & 2.28 & 1.19 \\
\hline & $\begin{array}{c}\text { Matrix } \\
\text { Assembly Secs. }\end{array}$ & 0.00 & 4.04 & 2.17 & 2.15 & 1.32 & 1.06 \\
\hline \multirow{4}{*}{$1 \mathrm{EM}$} & JCG Iter. & 24 & 24 & 24 & 24 & 24 & 24 \\
\hline & $\begin{array}{c}\text { JCG } \\
\text { CPU Secs. }\end{array}$ & 8511 & 7026 & 3722 & 1993 & 1081 & 586 \\
\hline & $\begin{array}{c}\text { Matrix } \\
\text { Build Secs. }\end{array}$ & 251.94 & 121.53 & 59.18 & 30.24 & 15.50 & 7.88 \\
\hline & $\begin{array}{c}\text { Matrix } \\
\text { Assembly Secs }\end{array}$ & 0.00 & 26.86 & 19.40 & 18.08 & 12.27 & 9.39 \\
\hline \multirow{4}{*}{$2 \mathrm{EM}$} & JCG Iter. & 24 & 24 & 24 & 24 & 24 & 24 \\
\hline & $\begin{array}{c}\text { JCG } \\
\text { CPU Secs. }\end{array}$ & 7581 & 6034 & 3135 & 1791 & 918 & 484 \\
\hline & $\begin{array}{c}\text { Matrix } \\
\text { Build Secs. }\end{array}$ & 102.09 & 49.96 & 24.60 & 12.56 & 6.42 & 3.31 \\
\hline & $\begin{array}{c}\text { Matrix } \\
\text { Assembly Secs. }\end{array}$ & 0.00 & 19.60 & 8.78 & 8.12 & 5.62 & 4.20 \\
\hline
\end{tabular}


It is interesting to note the number of iterations per solve for each of the various wave equations is consistent between wave equation types except for the nodal scalar acoustic equation.

In each of the cases the total run time is decreased as the number of processors is increased. The total simulation time and speedup for each of the various wave equations are plotted in Figure 7.21 and Figure 7.22 respectively. 

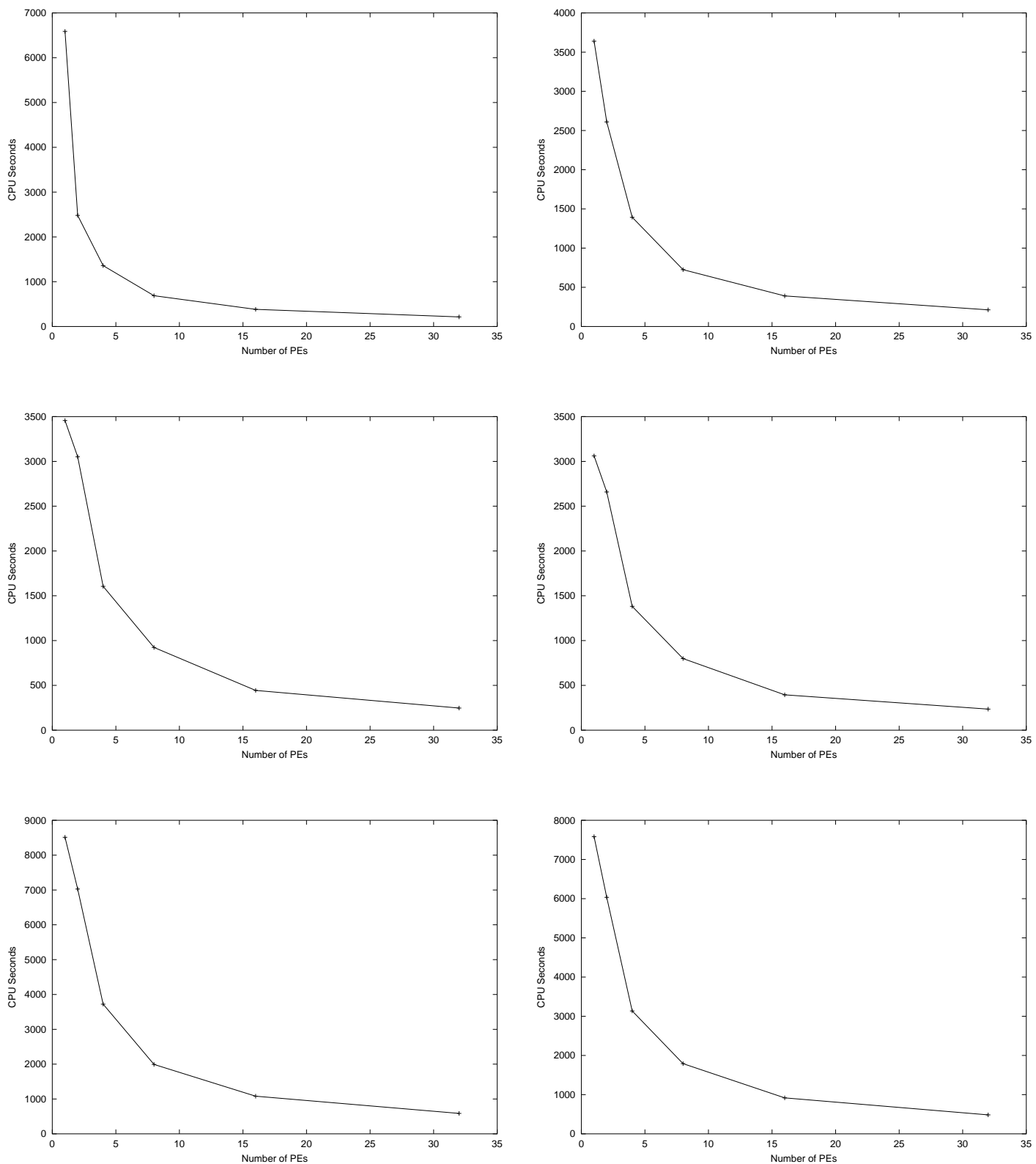

Figure 7.21: Hexahedral Grid Jacobi CG Run Times 

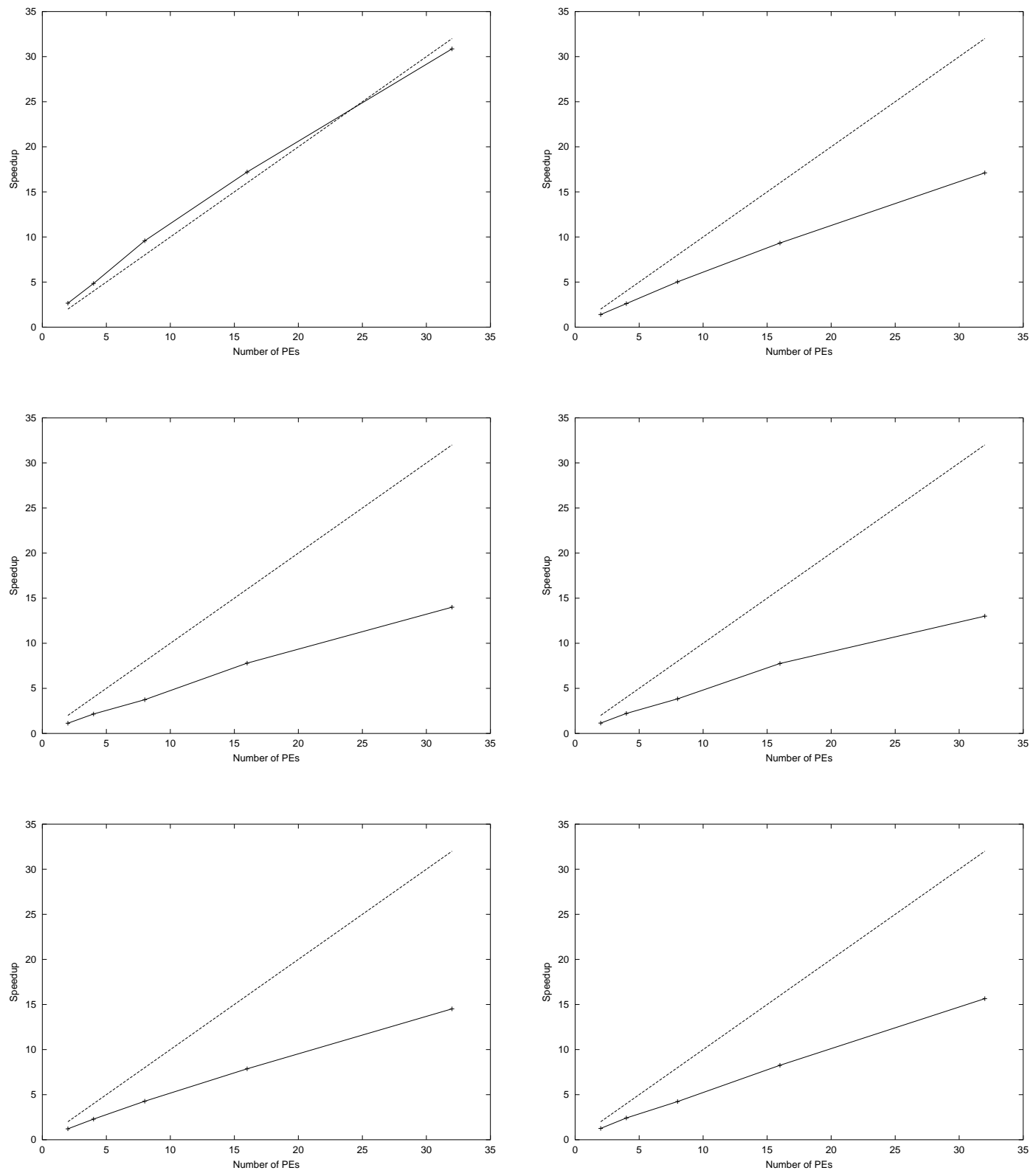

Figure 7.22: Hexahedral Grid Jacobi CG Speedup 


\section{Chapter 8}

\section{Results}

In the previous chapter simulations with known solutions were used for verification. In this chapter simulations with no known solution are presented. These simulations include pulse propagation in bent optical fibers, a photonic band gap device and a sonic band gap device. An issue with finite domains is the use of radiation or absorbing boundary conditions to truncate the physical domain. The majority of the theory surrounding these boundary conditions is for electrodynamics. The perfectly matched layer (PML) [64], [65] as well as the Sommerfeld boundary condition [14] are both used in general to provide absorbing boundary conditions. The PML is also used in acoustic wave simulations [66]. In this chapter the PML will be applied to the following simulations.

\subsection{Optical fiber waveguide}

The optical fiber [67], [68] is a ubiquitous method for communication of data for applications from phone calls to fighter planes. In this section a single mode step index 
optical fiber will be simulated. An optical fiber capable of carrying many modes is called a multimode fibers. These multimode fibers have core radii of 20-150 $\mu \mathrm{m}$.

In this section a single mode step index optical fiber will be simulated. To study the power loss of the propagated mode, the optical fiber will be bent at various angles. The TE01 mode of the optical fiber with a Gaussian envelope will be introduced into the optical fiber and the power will be recorded in several locations along the length of the fiber.

The three-dimensional full vectoral time-domain simulation of an optical fiber requires a large simulation domain even for physically short optical fibers. A typical single mode optical fiber has a core radius of 5-10 micrometers and a cladding radius of 120 micrometers which is in turn surrounded by buffers and mechanical cladding.

The overall length of the fiber can be in the 10s of kilometers before an amplifier is needed. In this simulation the total length of the optical fiber with PML region is 54.5 micrometers. The fiber will be represented by a cylindrical core surrounded by a cylindrical shell, representing the cladding. The radius of the core is 5 microns. A typical single mode optical fiber will have a cladding radius of 120 micrometers. This would make the simulation domain untenable. To reduce the simulation domain the radius of the cladding is extended 10 micrometers past the TE01 mode's extinction point resulting in a 30-micrometer fiber. The total length of the fiber is 52.5 micrometers, the bend radii are listed in Table 8.1.

The mode being propagated down the optical fiber is not the fundamental mode called HE11 but the next higher mode, the lowest transverse electric mode, TE01. This is done due to the non-zero propagation direction component of the HE11 mode. 
Table 8.1: Bend radii for the bent optical fibers

\begin{tabular}{|c|c|}
\hline Bend Angle & Bend Radius $\mu m$ \\
\hline \hline 30 & 100.27 \\
\hline 45 & 66.845 \\
\hline 60 & 55.134 \\
\hline
\end{tabular}

The TE01 mode for a step index fiber is defined in both the core and the cladding. In the cladding region the mode is an evanescent or radiating mode while in the core the mode is a propagating mode. The cylindrical geometry of the fiber results in a solution for the mode involving Bessel functions. The roots of the Bessel functions determine the modes. These roots are defined by the index of refraction in the core, $n_{1}$, and the cladding, $n_{2}$ and the wave number in the core, $k_{1}$ and the cladding, $k_{2}$. The TE and TM modes for the fiber are shown for the core (8.1) and cladding (8.2) with boundary condition $\vec{E} \times \hat{n}=0$; on $\Gamma$.

$$
\begin{array}{r}
\vec{E}_{r}(r, \theta, z)=-A_{m n} \frac{m}{\epsilon r} J_{m}(\kappa r)[-C \sin (m \theta)+D \cos (m \theta)] e^{-j \beta_{z} z} \\
\vec{E}_{\theta}(r, \theta, z)=A_{m n} \frac{\kappa}{\epsilon} J_{m}^{\prime}(\kappa r)[C \cos (m \theta)+D \sin (m \theta)] e^{-j \beta_{z} z} \\
\vec{E}_{z}(r, \theta, z)=0 \\
\vec{E}_{r}(r, \theta, z)=-B_{m n} \frac{m}{\epsilon r} K_{m}(\gamma r)[-C \sin (m \theta)+D \cos (m \theta)] e^{-j \beta_{z} z} \\
\vec{E}_{\theta}(r, \theta, z)=B_{m n} \frac{\gamma}{\epsilon} K_{m}^{\prime}(\gamma r)[C \cos (m \theta)+D \sin (m \theta)] e^{-j \beta_{z} z} \\
\vec{E}_{z}(r, \theta, z)=0
\end{array}
$$

The simulation uses the second order electrodynamic wave equation with a PML boundary condition shown in (8.3). The PML regions consist of a 30 zone region 
Table 8.2: Optical fiber parameters

\begin{tabular}{|c|c|c|c|c|c|c|}
\hline$n_{1}$ & $n_{2}$ & $\kappa$ & $\gamma$ & $\mathrm{V}$ & $e_{v}$ & $\omega$ \\
\hline \hline 1.471 & 1.456 & 0.602675 & 0.557111 & 4.25 & 2 & 4.053668 \\
\hline
\end{tabular}

with a cubic increase in conductivity. Separate PML regions are used for the core and cladding regions.

$$
\frac{\partial^{2} \vec{E}}{\partial t^{2}}=-\nabla \times \mu^{-1} \nabla \times \vec{E}-\left(\sigma_{E}+\mu^{-1} \sigma_{M} \epsilon\right) \frac{\partial \vec{E}}{\partial t}-\mu^{-1} \sigma_{M} \sigma_{E} \vec{E}
$$

The grid used for the simulations consists of 1.8 million elements with 5.5 million degrees of freedom. The grid with a bend angle of $30^{\circ}$ is shown in Figure 8.1.

A single timestep in the time series is shown for the optical fiber pulse simulations for a straight fiber, Figure 8.2, a 30 degree bent fiber, Figure 8.3, a 45 degree bent fiber, Figure 8.4, and a 60 degree bent fiber, Figure 8.5.

The power loss results are shown in Table 8.3.

Table 8.3: Curvature loss for the bent optical fibers

\begin{tabular}{|c|c|}
\hline Bend Angle & Measured \% Loss \\
\hline \hline 0 & 0.05 \\
\hline 30 & 0.47 \\
\hline 45 & 0.70 \\
\hline 60 & 0.85 \\
\hline
\end{tabular}

\subsection{Photonic band gap waveguide}

Photonic bandgap (PBG) devices [69] are the two and three-dimensional extensions of Bragg's Law to the optical regime. In this section a logically two-dimensional 


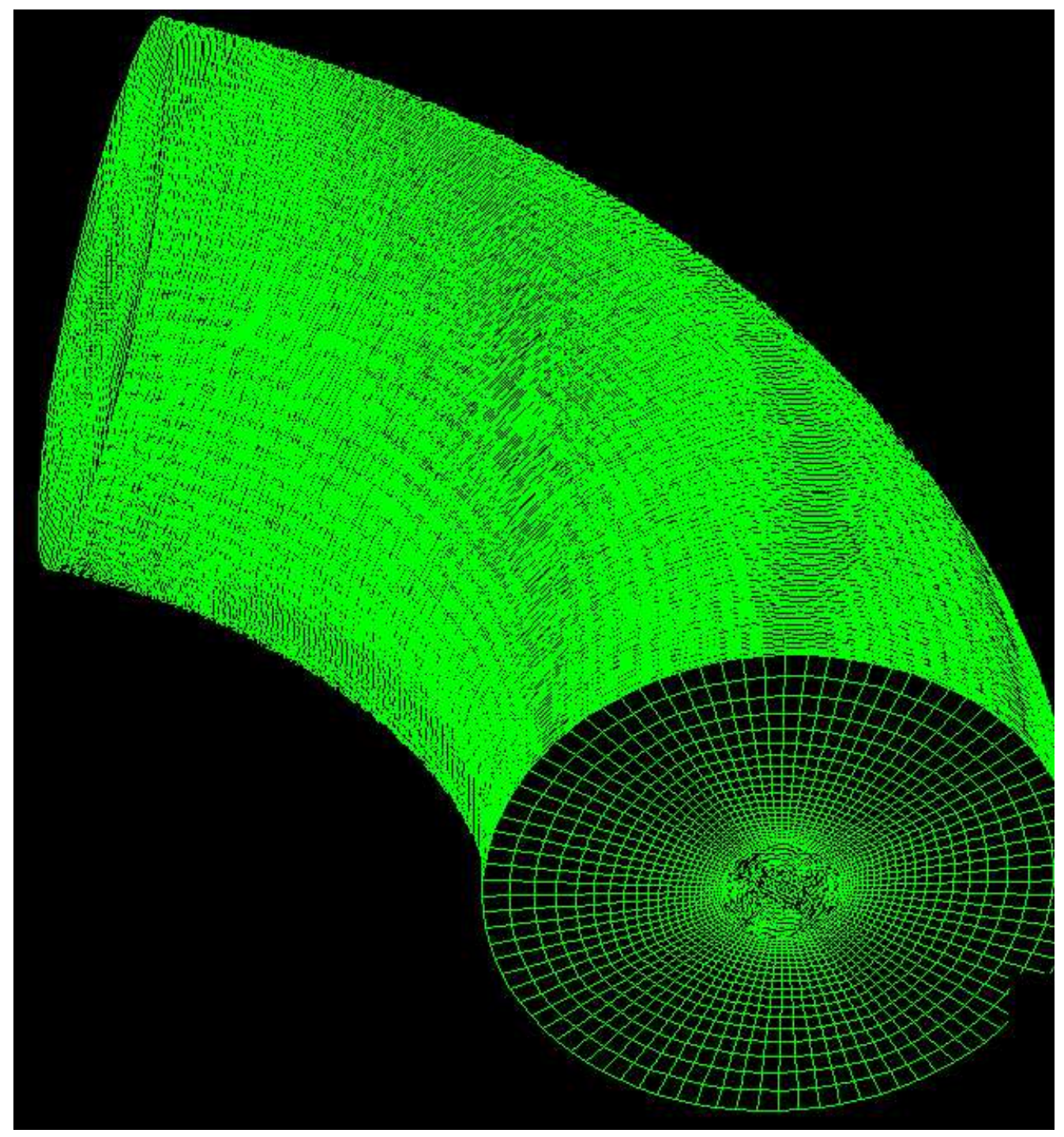

Figure 8.1: Bent optical fiber grid. 


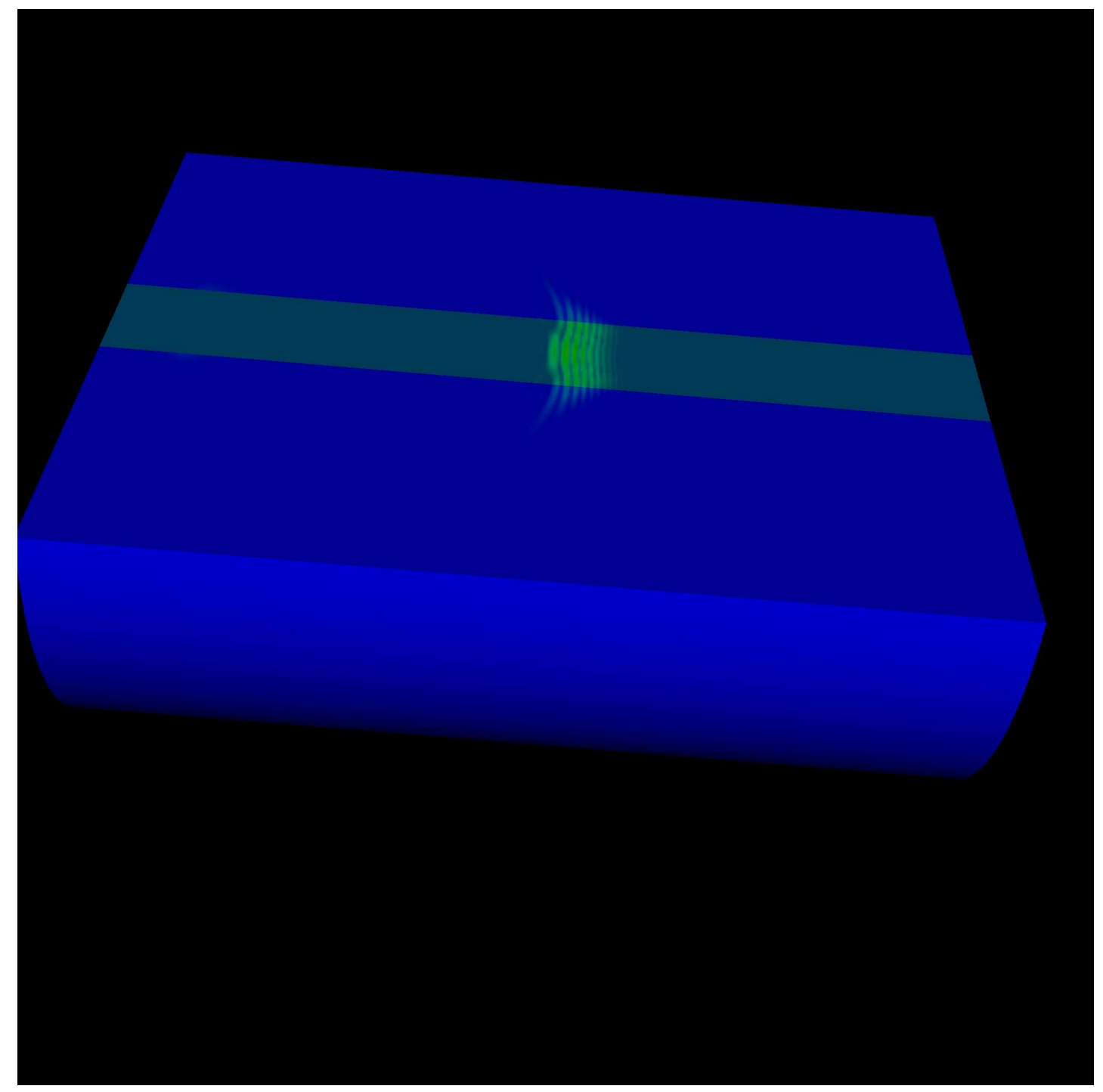

Figure 8.2: TE01 pulse propagating down a straight optical fiber. 


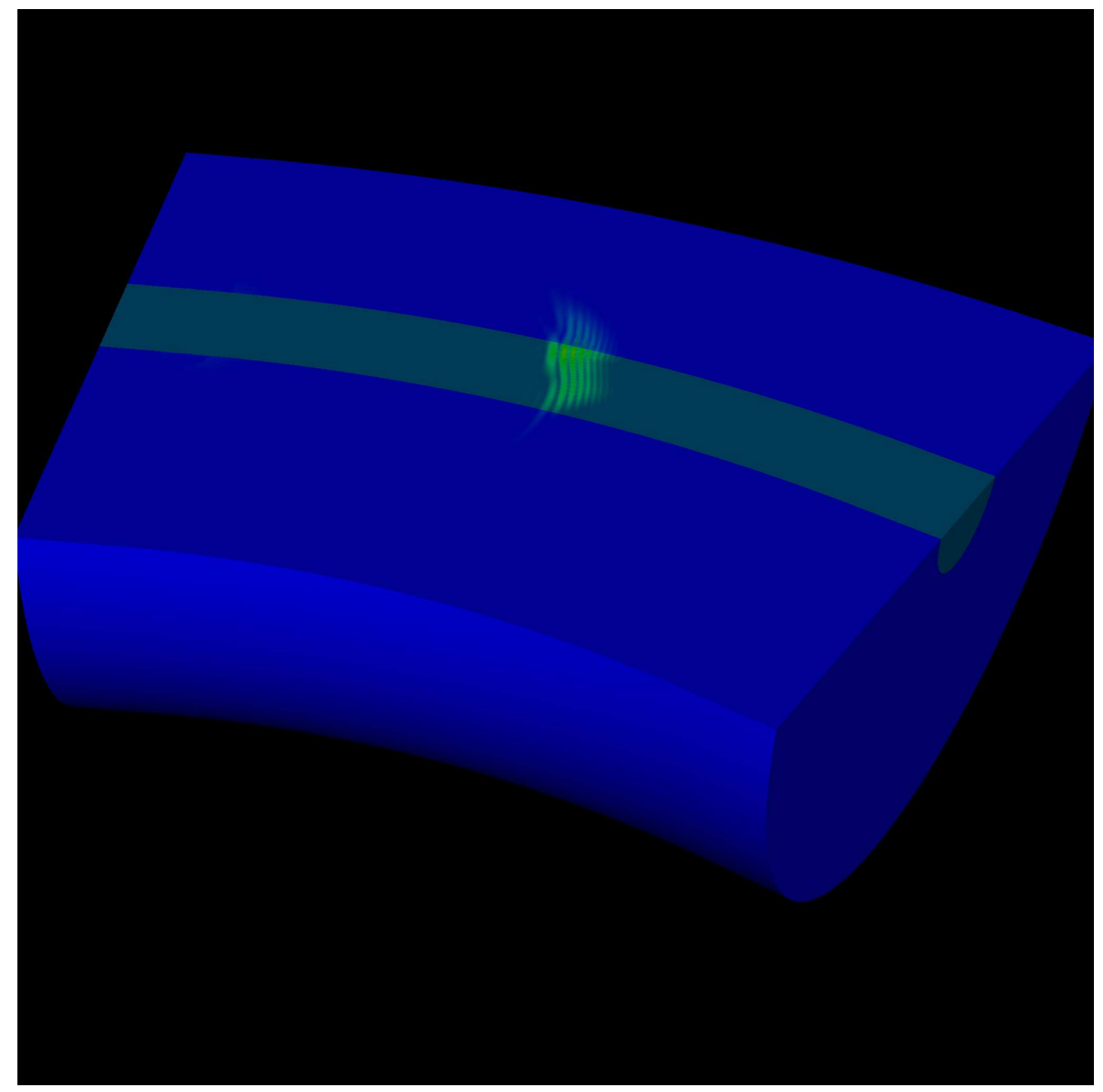

Figure 8.3: TE01 pulse propagating down an optical fiber with a 30 degree bend. 


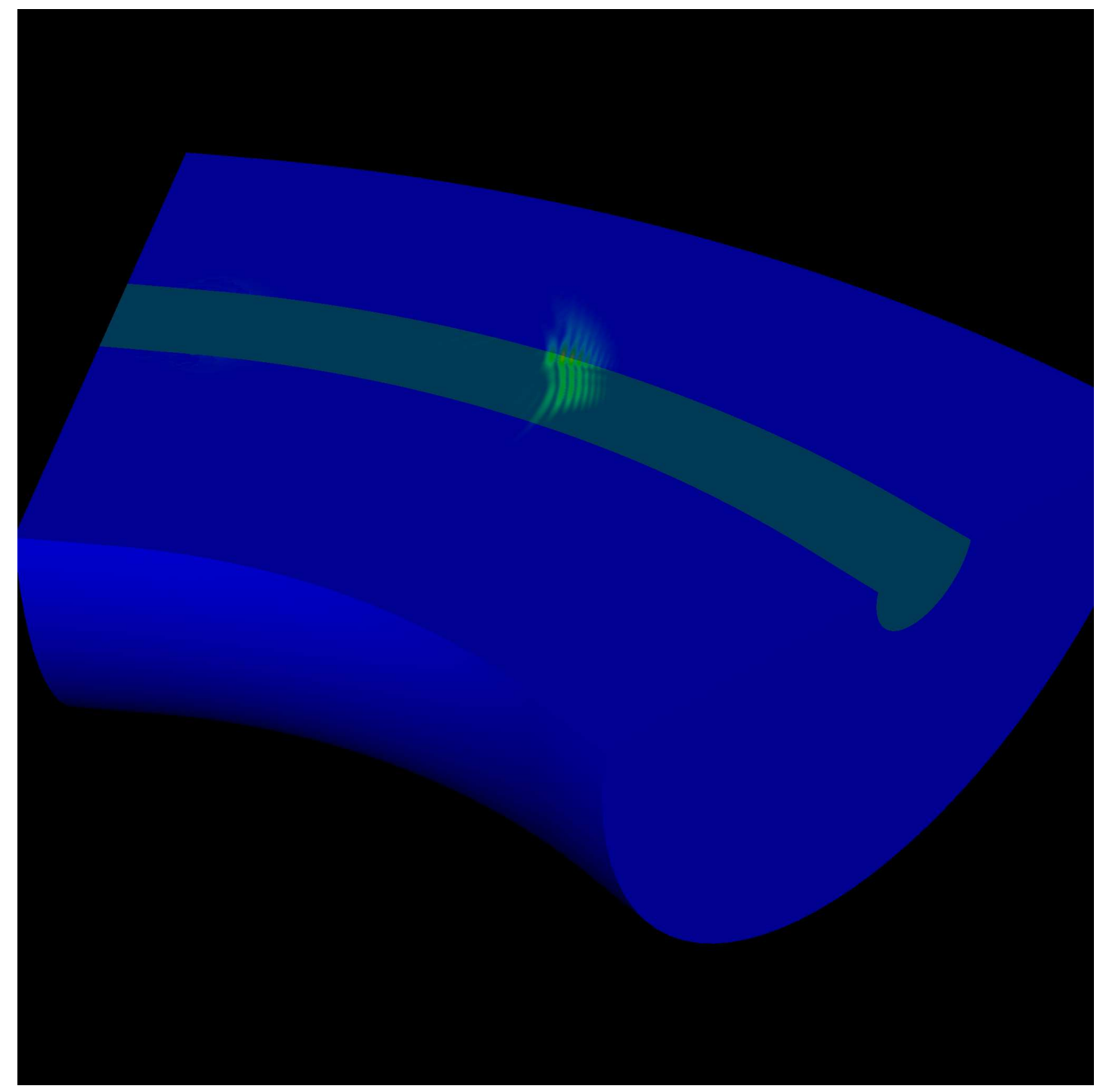

Figure 8.4: TE01 pulse propagating down an optical fiber with a 45 degree bend. 


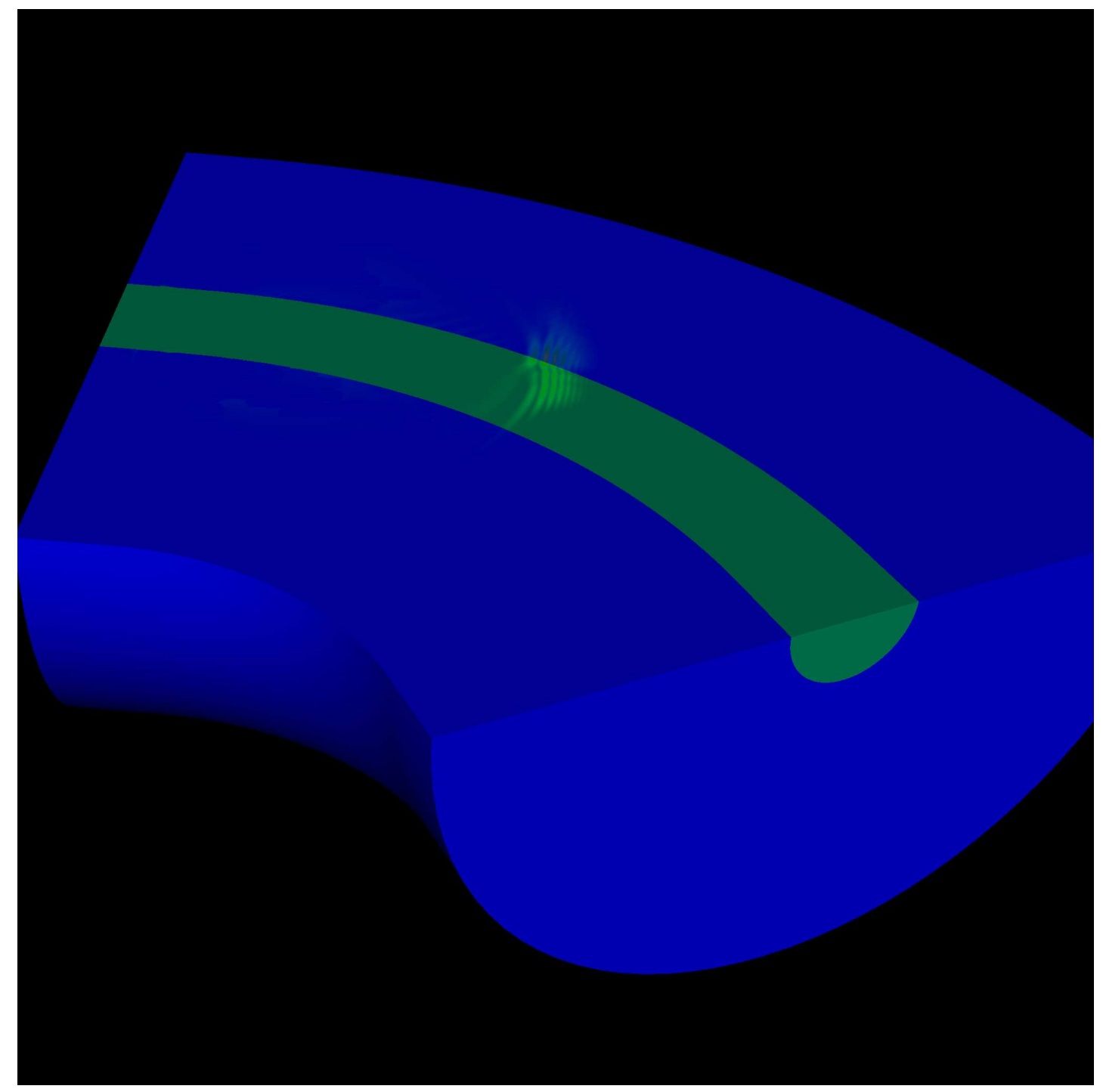

Figure 8.5: TE01 pulse propagating down an optical fiber with a 60 degree bend. 
simulation of a photonic bandgap waveguide will be analyzed. The PBG devices do not allow the propagation of energy through the domain for any incident wave within its stopgap or range of non-propagating frequencies. Introducing a defect in the structure allows a small range of wavelengths of light around a central defect frequency to propagate through the structure. Due to the nature of the photonic bandgap waveguide the light can be directed in arbitrary directions even ninety degree bends with very small energy loss. A ninety degree bend on the same scale in a purely optical device would result in the loss of a significant amount of energy. The ninety degree bend is possible due to Bragg diffraction occurring in all directions except where the defect exists. The energy has no direction to travel except within the defect waveguide. A mesh of the photonic band gap waveguide is shown in Figure 8.6. This mesh shows a two-dimensional section of a device with infinite Gallium-Arsenide (GaAs) rods arranged in a square array surrounded by air. Photonic structures such as these are characterised by the ratio of the rod radius $r$ to the rod spacing, or lattice constant, $a$. For this mesh the ratio $\frac{r}{a}=0.18$ is chosen for a frequency of $\lambda=1.55 \mu \mathrm{m}$ this gives a Transverse Magnetic (TM) stopgap of $\omega=0.302 \frac{2 \pi c}{a}$ to $\omega=0.443 \frac{2 \pi c}{a}$. In this simulation the TM modes are the modes with the electric field parallel to the GaAs columns.

The rod spacing for the geometry is $a=0.62 \mu \mathrm{m}$ with the relative electric permittivity for the GaAs rods $\epsilon_{r}=12$ and the relative electric permittivity for air $\epsilon_{r}=1.0$. To create the defect waveguide in the simulation several of the rods are defined to be air instead of GaAs. This structure is then excited by a TM mode on the left side of the mesh. 


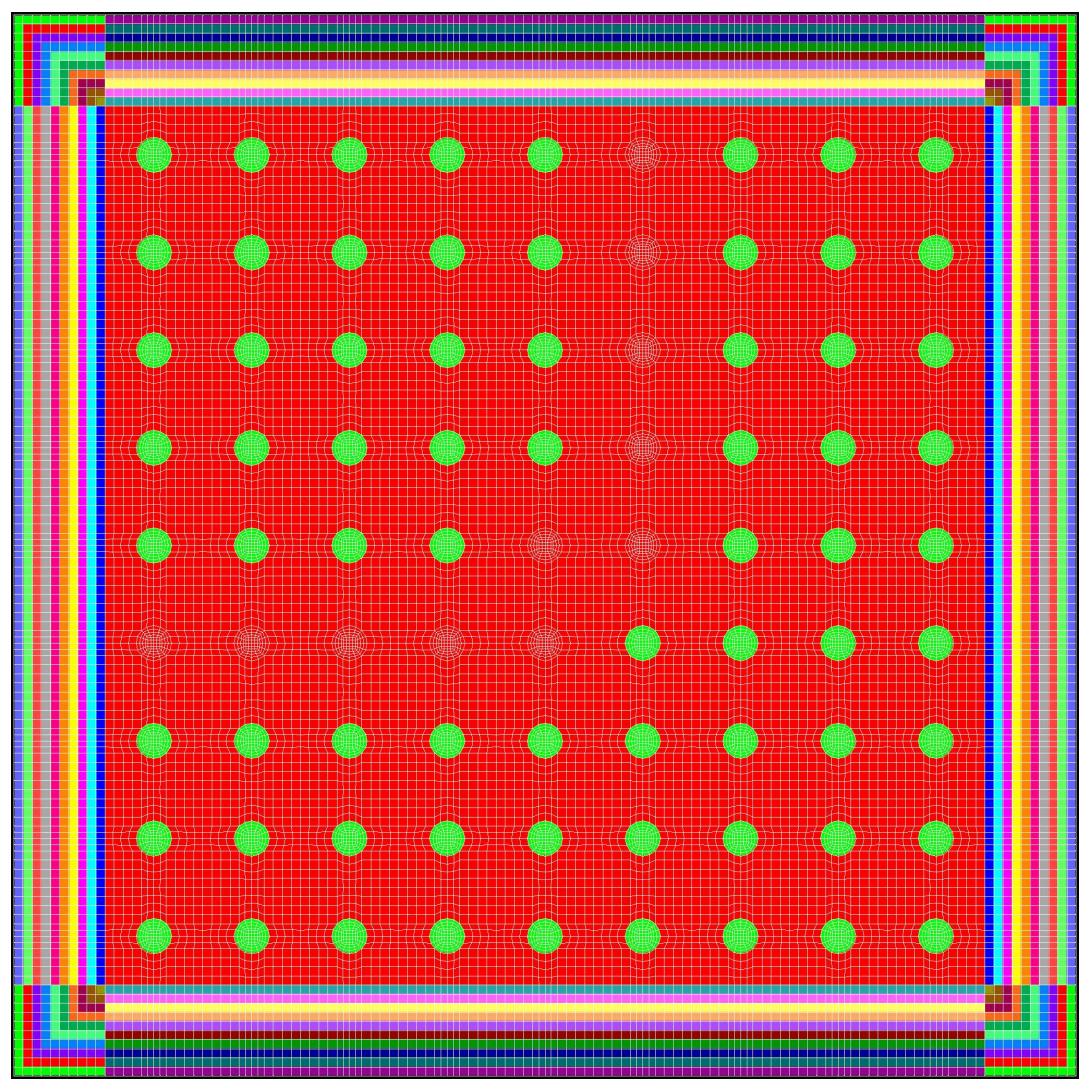

Figure 8.6: 2D photonic band gap mesh. 
Figure 8.7 shows the electric field magnitude for several timesteps of the simulation. As the wave approaches the bend, it takes several periods to proceed. This
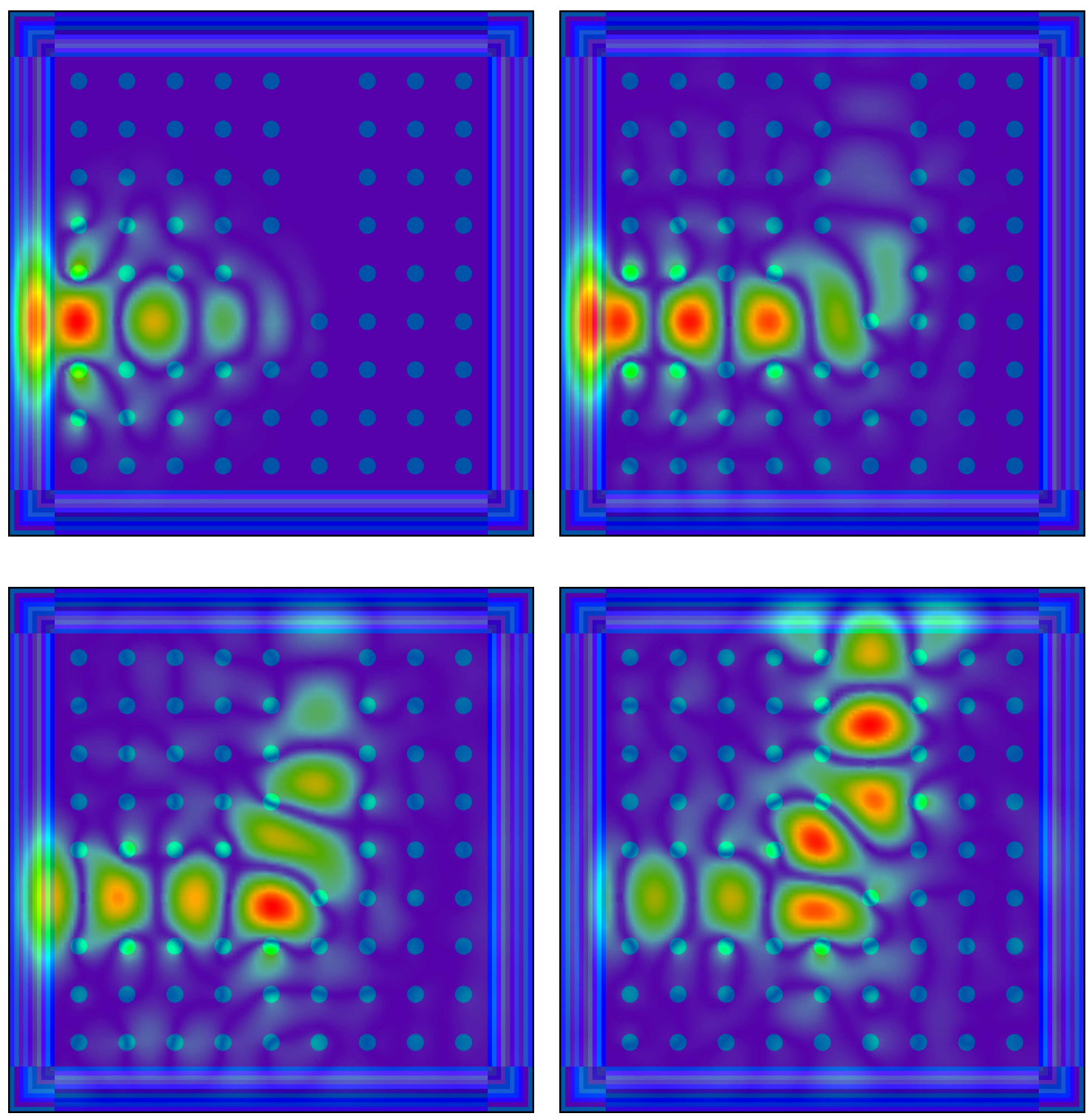

Figure 8.7: 2D Photonic band gap waveguide electric field magnitude.

is due to the diffraction occurring at the beginning of the bend. A wave encountering such an interface will take several periods before the diffraction constructively interferes enough to propagate the wave. 
The PBG is a periodic structure, to truncate the large physical domain the computationally domain is surrounded by a perfectly matched layer. These layers consist of regions with nonzero electric and magnetic conductivity tangential to the propagating direction. The conductivity tensors $\sigma_{E}$ and $\sigma_{M}$ have non-zero entries on the two diagonal entries orthogonal to the propagation direction and all other entries are zero. The conductivity increases from a very small initial value to a large value cubically. The PML region is terminated with a perfect electrical conducting boundary condition.

\subsection{Sonic band gap waveguide}

Sonic band gap (SBG) devices [70], [71] are the analog of the Photonic Band Gap devices in the region of sound frequencies 20-20,000 Hz discussed in Section 8.2. Instead of the optical regime the devices work in the region of sound frequencies. The mesh consists of cylindrical aluminium rods in air. Normally a simulation involving the acoustic wave equation would use the scalar pressure wave equation. The vector velocity wave equation was chosen to show the synergy between the electrodynamics PBG simulation and this SBG simulation. The structures are much larger than the photonic band gap structure presented previously, centimeters versus microns, however the same theory applies. Sonic band gap devices have been simulated using the scalar pressure acoustic wave equation for simulations of metal rods in air [72] with the same type of geometry as shown in Section 8.2. The transverse velocity in these simulations can be neglected resulting in the scalar pressure wave equation. Simulations of metal rods of one type embedded in a background metal of another 
type, e.g. aluminium rods in nickel, require the full elasticity equations [73].

In this section a sonic band gap device for aluminium rods in air will be simulated. The aluminium rods are considered to be infinite in length and have a diameter of $1.7875 \mathrm{~cm}$. They are spaced in a rectangular grid pattern at a distance of $5.5 \mathrm{~cm}$ between their centers. The simulation proceeds using the sound speeds for each of the materials. The true sound speeds at STP for air and aluminium are $330.14 \frac{\mathrm{m}}{\mathrm{s}}$ and $6420.0 \frac{\mathrm{m}}{\mathrm{s}}$ respectively. The relative sound speed in air is set to 1.0 while the relative sound speed in aluminium is 19.27 .

The domain is square surrounded by a fifteen layer PML. To show the synergy between the Photonic band Gap and Sonic band Gap devices, the vector velocity acoustic wave equation will be solved instead of the scalar pressure acoustic wave equation. With this wave equation the PML is directed in the normal direction. The normal component of the velocity field is attenuated as the wave travels into the PML region. The concept is the same as in the PBG case except for the direction of the dissipation. In the case of the electrodynamics simulation the conductivities were non-zero orthogonal to the propagation direction. In the acoustic wave equation the normal components of the field need to be attenuated. The PML tensor $q$ in (8.4) have nonzero entries for the diagonal component in the propagation direction and are zero elsewhere.

$$
\frac{\partial^{2} \vec{u}}{\partial t^{2}}=\nabla \nabla \cdot \vec{u}-2 \overline{\bar{q}} \frac{\partial \vec{u}}{\partial t}-\overline{\bar{q}}^{2} \vec{u}
$$

A port for the velocity mode to enter is defined on the left hand side of the grid. The velocity field will travel to the center of the grid, make a ninety-degree bend 


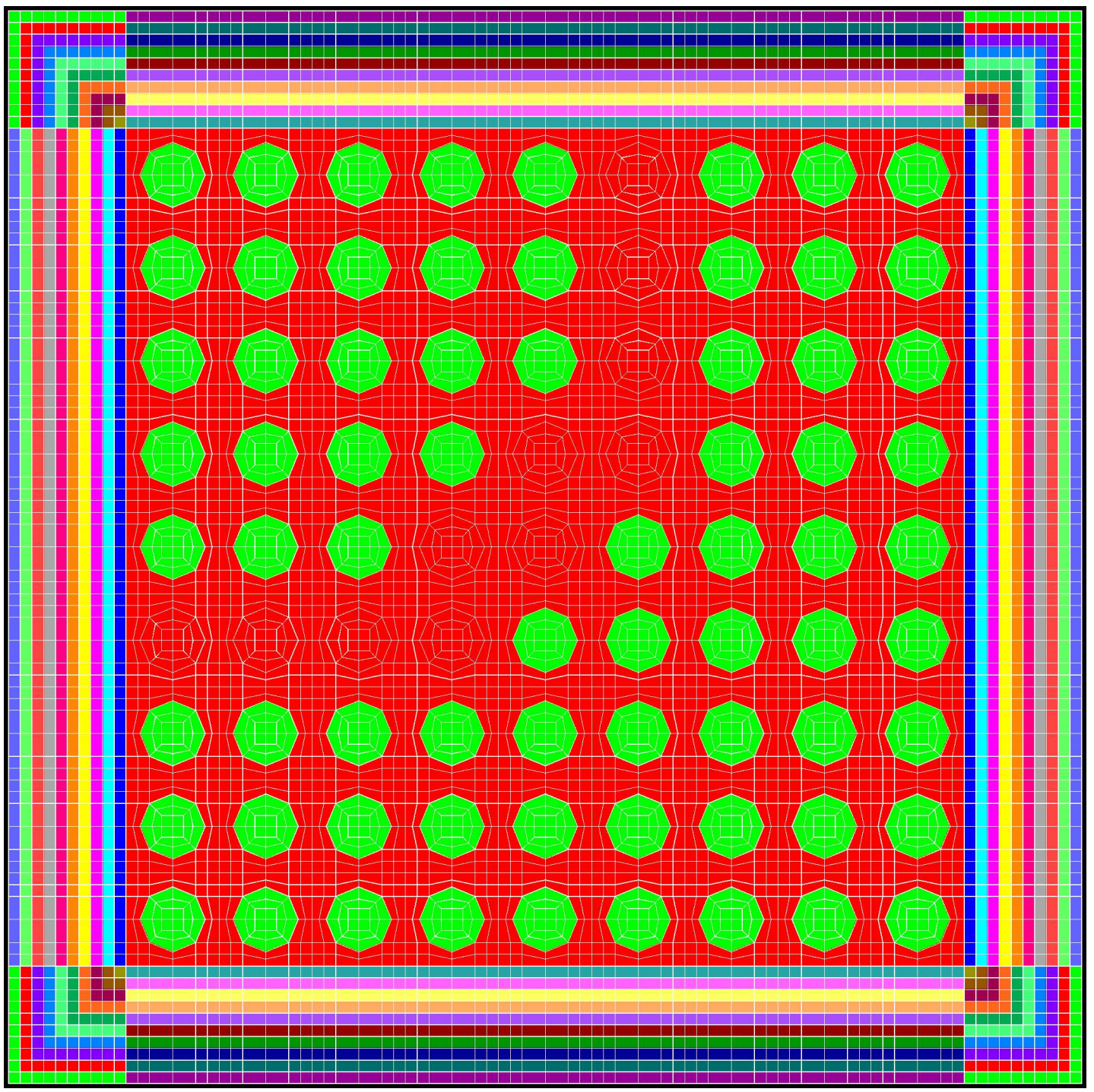

Figure 8.8: 2D sonic band gap mesh. 
upwards and be absorbed by the PML at the top. A time series of four times during the simulation is shown in Figure 8.9.
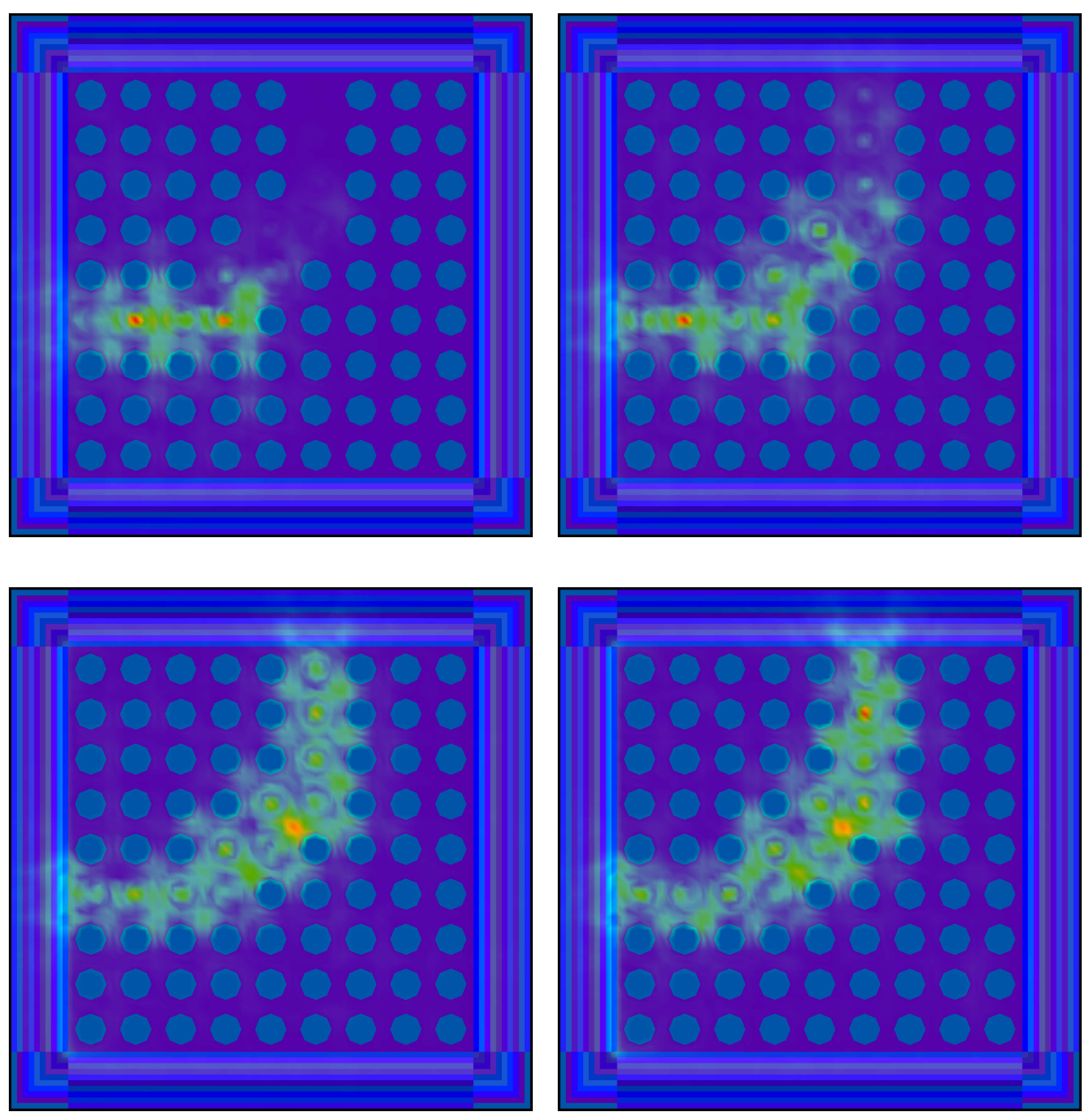

Figure 8.9: 2D Sonic band gap waveguide velocity magnitude. 


\section{Chapter 9}

\section{Conclusions}

In this dissertation a discrete differential forms finite element based framework has been presented. The discrete differential forms were implemented using the standard nodal and discontinuous volume centered finite elements coupled with the vector finite elements developed by Nédélec. Analyses of the operator accuracy, dispersion and stability as well as simulations prove that the method is second order accurate. Discrete vector operator analyses as well as discrete energy calculations show the method is conservative. Simulations were presented for structured and unstructured meshes in two and three-dimensions using three different types of finite elements. In each case the method was shown to be accurate, stable and conservative. Simulations for various types of linear wave equations were presented showing the method and operators can be applied to a wide variety of problems.

In this dissertation it has been shown that the object oriented framework for wave equations has the following properties.

- Discrete spaces that mimic continuous spaces. 
- Discrete differential operators that mimic continuous operators.

- Metric free discrete differential operators.

- Discrete spaces and differential operators that form exact sequences.

- Automatic conservation of energy, divergence free and curl free fields.

- Correct continuity of fields across material interfaces.

- Elimination of spurious modes.

- Well defined on structured and unstructured tessellations.

- Synergy between discrete simulation code for different differential forms.

- Second order accurate in space and time.

- Conditionally stable.

- Allows for scalar and tensor materials with spatial discontinuities.

Currently higher order basis functions as well as nonlinear materials and fields are being researched using this framework as a basis. Future research in the application of discrete differential forms to computational fluid dynamics and non-linear magnetohydrodynamics as well as hybrid boundary-element-finite-element or coupled discontinuous Galerkin-continuous Galerkin methods are all possible within the defined framework. 


\section{Bibliography}

1. W. L. Burke, Applied Differential Geometry. Cambridge, UK: Cambridge University Press, 1985.

2. F. Brezzi and M. Fortin, Mixed and Hybrid Finite Element Methods. New York, NY: Springer-Verlag, 1991.

3. C. Mattiussi, "An analysis of finite volume, finite element and finite difference methods using some concepts from algebraic topology," J. Comput. Phys., vol. 9, pp. 295-319, 1997.

4. K. S. Yee, "Numerical solution of initial boundary value problems involving Maxwell's equations in isotropic media," IEEE Transactions of Antennas and Propagation, vol. AP-14, no. 3, pp. 302-307, 1966.

5. A. A. Samarskii, V. F. Tishkin, A. Favosrkii, and M. Y. Shashkov, "Operational finite-difference schemes," Diff Eqns., vol. 17, pp. 854-862, 1981.

6. A. A. Samarskii, V. F. Tishkin, A. Favosrkii, and M. Y. Shashkov, "Employment of the reference-operator method in the finite-difference analogs of tensor operations," Diff Eqns., vol. 18, pp. 881-885, 1982.

7. M. Y. Shashkov, Conservative Finite-Difference Schemes on General Grids. CRC Press, 1995.

8. J. M. Hyman and M. Shashkov, "Natural discretizations for the divergence, gradient and curl on logically rectangular grids," Computers Math. Applic., vol. 33, no. 4, pp. 81-104, 1997.

9. J. M. Hyman and M. Shashkov, "Adjoint operators for the natural discretizations of the divergence, gradient and curl on logically rectangular grids," Applied Numrical Mathematics, vol. 5, pp. 13-442, 1997.

10. J. C. Nedelec, "Mixed finite elements in R3," Numer. Math., vol. 35, pp. 315331, 1980.

11. J. C. Nedelec, "A new family of mixed finite elements in R3," Numer. Math., vol. 50, pp. 57-81, 1986. 
12. P. A. Raviart and J. M. Thomas, "A Mixed Finite Element Method for $2^{\text {nd }}$ Order Elliptic Problems," in Mathematical Aspects of the Finite Element Method (I. Galligani and E. Mayera, eds.), vol. 606 of Lect. Notes. on Mathematics, pp. 293-315, Springer-Verlag, 1977.

13. A. Bossavit, Computational Electromagnetism Variational Formulations, Complementary, Edge Elements. San Diego, CA: Academic Press, 1998.

14. J. Jin, The Finite Element Method in Electromagnetics. John Wiley and Sons, 1993.

15. R. Hiptmair, "Discrete Hodge operators: An algebraic perspective," J. Electromagnteic Waves Appl., vol. 15, no. 3, pp. 343-344, 2001.

16. T. Weiland, "A discretization method for the solution of Maxwell's equations for six-component fields," Electron and Comminications $A E \ddot{U}$, vol. 31, no. 3, pp. 116-120, 1977.

17. M. Clemens and T. Weiland, "Discrete electromagnetism with the finite integration technique," Progress in Electromagnetic Research, vol. 32, pp. 65-87, 2001.

18. N. Madsen and R. Ziolkowski, "A 3 dimensional modified finite volume technique for maxwell's equations," Electromagnetics, vol. 10, no. 1, pp. 147-161, 1990.

19. N. Madsen, "Divergence preserving discrete surface integral method for maxwell's curl equations using non-orthogonal grids," J. Comput. Phys., vol. 119 , no. 1 , pp. 34-45, 1995.

20. R. Holland, V. Cable, and L. Wilson, "Finite-volume time-domain techniques for em scattering," IEEE Trans. Electromagnetic Compatibility, vol. 33, no. 4, pp. 281-294, 1995.

21. K. S. Yee and J. Chen, "Conformal hybrid finite difference time domain and finite volume time domain," IEEE Trans. Ant. Prop., vol. 42, no. 10, pp. 14501454, 1994.

22. D. A. White, Discrete Time Vector Finite Element Methods for Solving Maxwell's Equations on 3D Unstructured Grids. PhD thesis, University of California at Davis, September 1997.

23. C. Riley, D.J.; Turner, "VOLMAX: a solid-model-based, transient volumetric maxwell solver using hybrid grids," IEEE Antennas and Propagation Magazine, vol. 39, no. 1, pp. 20-33, 1997.

24. B. Stroustrup, $C++$ Programming Language. Addison-Wesley, Reading, MA, 1991.

25. G. V. R. et al., "Python home page." http://www.python.org, 2003. 
26. S. Balay, W. D. Gropp, L. C. McInnes, and B. F. Smith, "Efficient management of parallelism in object oriented numerical software libraries," in Modern Software Tools in Scientific Computing (E. Arge, A. M. Bruaset, and H. P. Langtangen, eds.), pp. 163-202, Birkhauser Press, 1997.

27. S. Balay, K. Buschelman, W. D. Gropp, D. Kaushik, L. C. McInnes, and B. F. Smith, "PETSc home page." http://www.mcs.anl.gov/petsc, 2002.

28. S. Balay, W. D. Gropp, L. C. McInnes, and B. F. Smith, "PETSc users manual," Tech. Rep. ANL-95/11 - Revision 2.1.3, Argonne National Laboratory, 2002.

29. E. Butkov, Mathematical Physics. Reading, MA: Addison-Wesley, 1968.

30. C. A. Balanis, Advance Engineering Electromagnetics. New York, NY: John Wiley and Sons, 1989.

31. J. G. Harris, Linear Elastic Waves. Cambridge, UK: Cambridge University Press, 2001.

32. P. L. Gould, Introduction to Linear Elasticity. New York, NY: Springer-Verlag, 1983.

33. J. D. Achenbach, Wave Propagation in Solids. Elsevier Science Publishers, 1975.

34. D. Hestenes and G. Sobczyk, Clifford Algebra to Geometric Calculus. Dordrecht, Holland: Kluwer Academic Publishing, 1992.

35. S. Carrol, "Lecture notes on general relativity," Tech. Rep. gr/qc 9712019, University of Chicago, http://nedwww.ipac.caltech.edu/level5/March01/Carroll3/Carroll_contents.html, 1997.

36. S. C. Brenner and L. R. Scott, The Mathematical Theory of Finite Element Methods. New York, NY: Springer-Verlag, 1994.

37. B. D. Reddy, Introductory Functional Analysis : With Applications to Boundary Value Problems and Finite Elements. New York, NY: Springer-Verlag, 1998.

38. R. Hiptmair, "Multigrid method for 'Maxwell's equations," SIAM J. Numer. Anal., vol. 38, no. 1, pp. 204-225, 1998.

39. S. G and G. Fix, An Analysis of the Finite Element Method. Prentice-Hall, 1973.

40. P. G. Ciarlet, The Finite Element Method for Elliptic Problems. North-Holland, 1978.

41. A. B. T Tarhasaari, L Kettunen, "Some realizations of a discrete hodge operator: a reinterpretation of finite element techniques for em field analysis.," IEEE Trans. Mag., vol. 35, no. 3, pp. 1494-1497, 1999.

42. G. C. Cohen, Higher-Order Numerical Methods for Transient Wave Equations. New York, NY: Springer-Verlag, 2001. 
43. Z. J. Cendes, "Vector finite elements for electromagnetic field calculations," vol. 27, no. 5, pp. 3958-3966, 1991.

44. J. W. Demmel, J. R. Gilbert, and X. S. Li, "Superlu users' guide," tech. rep., U. C. Berkeley, Berkeley, CA., 1997.

45. O. Axelsson, Iterative Solution Methods. Cambridge, UK: Cambridge University Press, 1996.

46. L. Lapidus and G. Pinder, Numerical Solution of the Partial Differential Equations in Science and Engineering. Wiley, 1982.

47. J. M. Koning, D. A. White, and G. H. Rodrigue, "Scalable preconditioned conjugate gradient inversion of vector finite element mass matrices," Journal of Comp. and Appl. Math., vol. 123, pp. 307-321, 2000.

48. J. C. Tannehill, D. A. Anderson, and R. H. Pletcher, Computational Fluid Mechanics and Heat Transfer. Washington, DC: Taylor and Francis, second ed., 1997.

49. S. Warren and W. Scott, "An investigation of numerical dispersion in the vector finite element method using quadrilateral elements," IEEE Trans. Ant. Prop., vol. 42, no. 11, pp. 1502-1508, 1994.

50. S. Warren and W. Scott, "Numerical dispersion in the finite element method using triangular edge elements," Opt. Tech. Lett., vol. 9, no. 6, pp. 315-319, 1995.

51. D. A. White, "Numerical dispersion of a vector finite element method on skewed hexahedral grids," Commun. Numer. Meth. Engng., vol. 16, pp. 47-55, 2000.

52. A. Chalmers and J. Tidmus, Practical Parallel Processing : An Introduction to Problem Solving in Parallel. London, England: International Thompson Computer Press, 1996.

53. U. of Mannheim, U. of Tennessee, and NERSC/LBNL, "Top500 home page." http://www.top500.org, 2002.

54. M. Snir, S. W. Otto, S. Huss-Lederman, D. W. Walker, and J. Dongarra, MPI : The Complete Reference. Cambridge, MA: The MIT Press, 1996.

55. R. Falgout, G. Castilla, E. Chow, A. Cleary, V. E. Henson, J. Jones, M. Lambert, B. Lee, J. Painter, C. Tong, T. Treadway, P. Vassilevski, and U. M. Yang, "Hypre home page." http://www.llnl.gov/casc/hypre, 2002.

56. D. Abrahams, "Boost python home page." http://cvs.sourceforge.net/cgibin/viewcvs.cgi/*checkout*/boost/boost/libs/python/doc/index.html, 2002.

57. D. M. Beazly, "Swig home page." http://www.swig.org/, 2002. 
58. G. Karypis and V. Kumar, "Multilevel k-way partitioning scheme for irregular graphs," Tech. Rep. TR 95-064, Dept. of Computer Science, University of Minnesota, 1995.

59. G. Karypis and V. Kumar, "METIS: Serial unstructured graph partitioning and sparse matrix ordering system." http://wwwusers.cs.umn.edu/ karypis/metis/index.html, 2003.

60. G. Karypis and V. Kumar, "ParMETIS: Parallel unstructured graph partitioning and sparse matrix ordering system." http://wwwusers.cs.umn.edu/ karypis/metis/parmetis/index.html, 2003.

61. XYZ Scientific Applications Inc., "Truegrid home page." http://www.xyz.com/, 2002.

62. CFDRC, "CFD-Micromesh home page." http://www.cfdrc.com/datab/software/ micromesh/micromesh.html, 2002.

63. B. Joe, "GEOMPACK - A software package for the generation of meshes using geometrical algorithms," Adv. Engr. Software, vol. 13, pp. 325-331, 1991.

64. J. Berenger, "A perfectly matched layer for the absorption of electromagnetic waves," J. Comput. Phys., vol. 114, no. 2, pp. 185-200, 1994.

65. J. Berenger, "Three dimensional perfectly matched layer for the absorption of electromagnetic waves," J. Comput. Phys., vol. 127, no. 2, pp. 363-379, 1996.

66. Q. Qi and T. Geers, "Evaluation of the perfectly matched layer for computational acoustics," J. Comput. Phys., vol. 139, pp. 166-183, 1998.

67. D. Marcuse, Theory of Dielectric Optical Waveguides. New York, NY: Academic Press, 1974.

68. N. S. K. J. J. Burke, Optical Waveguides. New York, NY: Academic Press, 1972.

69. J. D. Joannopoulus, R. D. Meade, and J. N. Winn, Photonic Crystals : Molding the Flow of Light. Princeton, NJ: Princeton University Pressl, 1995.

70. J. V. Sanchez-Perez, D. Caballero, R. Martinez-Sala, C. Rubio, J. SanchezDehesa, F. Meseguer, J. Llinares, and F. Galvez, "Sound attenuation by a twodimensional array of rigid cylinders," Physical Review Letters, vol. 80, no. 24, pp. 5325-5328, 1998.

71. P. Ball, "Sculpted sound," New Scientist, pp. 32-35, March 2002.

72. M. M. Sigalas and E. N. Economou, "Attenuation of multiple-scattered sound," EuroPhysics Letters, vol. 36, no. 4, pp. 241-246, 1996.

73. M. Kushawa, P.Halevi, G. Martinez, L. Dobrzynski, and B. Djafar-Rouhani, "Theory of acoustic band structure of periodic elastic composites," vol. 49, no. 4 , pp. 2313-2322, 1994. 


\section{Appendix A}

\section{Reduced Unit Formulation for}

\section{Electrodynamics}

In this appendix I discuss the reduced units. The equations (2.28) (2.27) can be rewritten so that $c=1$ and $\epsilon$ and $\mu$ are relative to the the vacuum permittivity and permeability. Using reduced units requires that $\sigma_{E}$ also be transformed into these new units. This is important because the choice of $\sigma_{E}$ sets the resistance.

\section{A.0.1 Second Order Reduced Wave Equation}

Starting with the relative $\epsilon=\epsilon_{r} \epsilon_{0}$ and $\mu=\mu_{r} \mu_{0}$ and using $c=\frac{1}{\sqrt{\epsilon_{0} \mu_{0}}}$, multiply (2.28) by $\mu_{0}$, this gives:

$$
\frac{1}{c^{2}} \frac{\partial^{2} \epsilon_{r} \vec{E}}{\partial t^{2}}=-\nabla \times \mu_{r}^{-1} \nabla \times \vec{E}-\mu_{0} \sigma_{E} \frac{\partial \vec{E}}{\partial t}-\mu_{0} \frac{\partial \vec{J}}{\partial t}
$$

Choose $c=1$, this is the same as choosing a new time variable, $\tau=c t$. Note that the units of $\tau$ are now in meters. This time transformation leaves the new time variable $\tau$ with units of $[m]$ instead of $[s]$. The chain rule for the time derivatives is 
shown in (A.2).

$$
\frac{\partial x}{\partial t}=\frac{\partial x}{\partial \tau} \frac{\partial \tau}{\partial t}=c \frac{\partial x}{\partial \tau}
$$

Substituting (A.2) into (A.1) gives:

$$
\frac{1}{c^{2}} c^{2} \frac{\partial^{2} \epsilon_{r} \vec{E}}{\partial \tau^{2}}=-\nabla \times \mu_{r}^{-1} \nabla \times \vec{E}-c \mu_{0} \sigma_{E} \frac{\partial \vec{E}}{\partial \tau}-c \mu_{0} \frac{\partial \vec{J}}{\partial \tau}
$$

which reduces to:

$$
\frac{\partial^{2} \epsilon_{r} \vec{E}}{\partial \tau^{2}}=-\nabla \times \mu_{r}^{-1} \nabla \times \vec{E}-\sqrt{\frac{\mu_{0}}{\epsilon_{0}}} \sigma_{E} \frac{\partial \vec{E}}{\partial \tau}-\sqrt{\frac{\mu_{0}}{\epsilon_{0}}} \frac{\partial \vec{J}}{\partial \tau}
$$

Now if the mesh is constructed in arbitrary units $\tilde{x}=b x$, the chain rule for this becomes:

$$
\frac{\partial \vec{E}}{\partial x}=\frac{\partial \vec{E}}{\partial \tilde{x}} \frac{\partial \tilde{x}}{\partial x}=b \frac{\partial \vec{E}}{\partial \tilde{x}}
$$

This transforms the curl-curl term:

$$
-\nabla \times \mu_{r}^{-1} \nabla \times \vec{E} \rightarrow-b^{2} \nabla \times \mu_{r}^{-1} \nabla \times \vec{E}
$$

Choosing a different time transformation $\tau=$ at gives:

$$
\frac{1}{c^{2}} \frac{a^{2}}{b^{2}} \frac{\partial^{2} \epsilon_{r} \vec{E}}{\partial \tau^{2}}=-\nabla \times \mu_{r}^{-1} \nabla \times \vec{E}-\mu_{0} \sigma_{E} \frac{a}{b^{2}} \frac{\partial \vec{E}}{\partial \tau}-\mu_{0} \frac{a}{b^{2}} \frac{\partial \vec{J}}{\partial \tau}
$$

Choosing $a=c b$ the final result is achieved:

$$
\begin{gathered}
\tau=c b t \\
\frac{\partial^{2} \epsilon_{r} \vec{E}}{\partial \tau^{2}}=-\nabla \times \mu_{r}^{-1} \nabla \times \vec{E}-\sqrt{\frac{\mu_{0}}{\epsilon_{0}}} \frac{\sigma_{E}}{b} \frac{\partial \vec{E}}{\partial \tau}-\sqrt{\frac{\mu_{0}}{\epsilon_{0}}} \frac{1}{b} \frac{\partial \vec{J}}{\partial \tau} \\
\vec{E} \times \hat{n}=0 \text { on } \Gamma_{D} \\
\nabla \times \vec{E} \times \hat{n}=\frac{a}{b} \frac{\partial J(t)}{\partial t} \text { on } \Gamma_{N}
\end{gathered}
$$

This gives the relative conductivity, using $Z_{0}=\sqrt{\frac{\mu_{0}}{\epsilon_{0}}}=377 \mathrm{ohms}$,

$$
\sigma_{r}=\sigma_{E} \frac{Z_{0}}{b}
$$




\section{A.0.2 First Order Reduced Wave Equation}

Using the same transformations as in the section above the first order reduced wave equation becomes:

$$
\begin{array}{r}
\frac{1}{c} \frac{\partial \epsilon_{r} \vec{E}}{\partial \tau}=-\nabla \times \mu_{r}^{-1} \vec{B}-\frac{\mu_{0} \sigma_{E}}{b} \vec{E}-\frac{\mu_{0}}{b} \vec{J} \\
c \frac{\partial \mu_{r}^{-1} \vec{B}}{\partial \tau}=-\mu_{r}^{-1} \nabla \times \vec{E} \\
\vec{E} \times \hat{n}=0 \text { on } \Gamma_{D} \\
\nabla \times \vec{E} \times \hat{n}=\frac{a}{b} \frac{\partial J(t)}{\partial t} \text { on } \Gamma_{N}
\end{array}
$$




\section{Appendix B}

\section{Vector Identities}

This appendix lists the vector identities used throughout the dissertation. The values in the properties include scalars: $\alpha$, vectors: $\vec{A}, \vec{B}$ and $\vec{C}$ and tensors: $\overline{\bar{T}}$.

$$
\begin{gathered}
\vec{A} \cdot(\vec{B} \times \vec{C})=\vec{B} \cdot(\vec{C} \times \vec{A})=\vec{C} \cdot(\vec{A} \times \vec{B}) \\
\alpha \nabla \cdot \vec{A}=\nabla \cdot(\alpha \vec{A})-\vec{A} \cdot \nabla \alpha \\
\nabla \cdot(\vec{A} \times \vec{B})=\vec{B} \cdot \nabla \times \vec{A}-\vec{A} \cdot \nabla \times \vec{B} \\
\nabla \cdot(\overline{\bar{T}} \cdot \vec{A})=(\nabla \cdot \overline{\bar{T}}) \cdot \vec{A}+\overline{\bar{T}} \cdot \nabla \vec{A} \\
\operatorname{Tr}(\nabla \vec{A})=\nabla \cdot \vec{A} \\
\nabla \cdot \nabla \vec{A}=\nabla \nabla \cdot \vec{A}-\nabla \times \nabla \times \vec{A}
\end{gathered}
$$


Approved for public release; further dissemination unlimited 


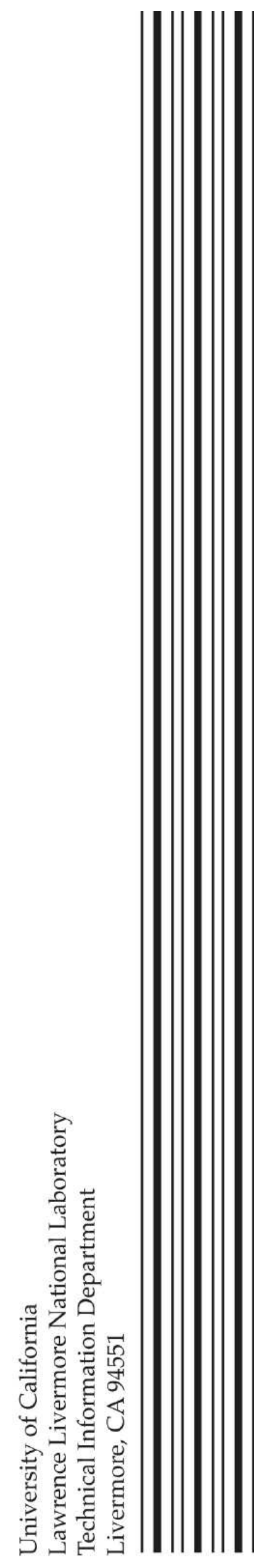

